Aus dem Institut für Rechtsmedizin der Ludwig-Maximilians-Universität zu München

Vorstand: Prof. Dr. med. W. Eisenmenger

\title{
Der Plötzliche Säuglingstod
}

Untersuchungen eigener Fälle am Institut für Rechtsmedizin der LudwigMaximilians-Universität zu München aus den Jahren 1999 bis 2001

\author{
Dissertation \\ zum Erwerb des Doktorgrades der Medizin \\ an der Medizinischen Fakultät der \\ Ludwig-Maximilians-Universität zu München
}

vorgelegt von

Bettina Michaela Zinka

aus

Aachen

2004 


\section{Mit Genehmigung der Medizinischen Fakultät der Universität München}

1. Berichterstatter:

2. Berichterstatter:

Mitberichterstatter:

Mitbetreuung durch die promovierte Mitarbeiterin:

Dekan:

Tag der mündlichen Prüfung:
Prof. Dr. med. Randolph Penning Prof. Dr. med. B. Koletzko

Prof. Dr. med. J. Hasford Prof. Dr. med. W. G. Locher

Dr. med. Elisabeth Rauch

Prof. Dr. med. Dr. h. c. Klaus Peter

04. März 2004 
Für Marc 


\section{INHALTSVERZEICHNIS}

\section{EINLEITUNG UND LITERATURÜBERSICHT S. 1}

1.1. Definition S. 1

1.2. Sicht des Plötzlichen Säuglingstodes bis zum Ende des

19. Jahrhunderts

S. 2

1.2.1. Akzidentelles Ersticken $\quad$ S. 3

$\begin{array}{ll}\text { 1.2.2. Asthma thymicum } & \text { S. } 4\end{array}$

1.2.3. Status thymico-lymphaticus S. 5

1.3. Der plötzliche Säuglingstod ab dem 20. Jahrhundert S. 6

1.4. Neue Theorien über die Ursachen des SIDS

1.4.1. Periodische, zentrale und obstruktive Apnoen $\quad$ S. 8

1.4.2. Das QT-Syndrom $\quad$ S. 10

1.4.3. Zytomegalievirus-Infektion $\quad$ S. 11

1.5. Epidemiologie und Risikofaktoren $\quad$ S. 11

1.5.1. SIDS-Inzidenz und Anteil an der postneonatalen Gesamtmortalität

S. 12

1.5.2. Tages- und jahreszeitliche Verteilung $\quad$ S. 15

$\begin{array}{ll}\text { 1.5.3. Geschlechtsverteilung } & \text { S. } 16\end{array}$

1.5.4. Altersverteilung $\quad$ S. 16

1.5.5. Auffindesituation, Bauchlage und „Back to sleep“-Kampagnen $\quad$ S. 18

1.5.6. Postnatale Entwicklung der SIDS-Fälle S. 19

1.5.7. Ethnische Abstammung $\quad$ S. 20

$\begin{array}{ll}\text { 1.5.8. Weitere Risikofaktoren } & \text { S. } 21\end{array}$

1.6. Die Obduktion S. 23

1.6.1. SIDS-typische Obduktionsbefunde $\quad$ S. 24

$\begin{array}{ll}\text { 1.6.2. SIDS versus Tötungsdelikt } & \text { S. } 28\end{array}$

1.7. Die BMBF-Studie „Plötzlicher Säuglingstod“ $\quad$ S. 30

$\begin{array}{ll}\text { 1.7.1. Studienteile und-ziele } & \text { S. } 30\end{array}$

$\begin{array}{ll}\text { 1.7.2. Studiendesign } & \text { S. } 31\end{array}$

1.7.3. Ein- und Ausschlußkriterien $\quad$ S. 32

$\begin{array}{ll}\text { 1.8. Ziele dieser Arbeit } & \text { S. } 32\end{array}$

2. MATERIAL UND METHODEN S. 33

2.1. Epidemiologische Datenerhebung S. 33

2.2. Histologische Untersuchungen S. 34

2.2.1. Entnommenes Gewebe und Fixation $\quad$ S. 34

2.2.2. Zuschnitt, Einbettung und Schnitt des Gewebes S. 34

$\begin{array}{ll}\text { 2.2.3. Färbungen } & \text { S. } 35\end{array}$

2.2.4. Mikroskopie S. 37

2.3. Bakteriologie S. 38

$\begin{array}{ll}\text { 2.4. Virologie S. } 38 & \end{array}$

2.5. Toxikologie, Ethanolbestimmung und

Begleitstoffanalyse 
2.6. Weitere Untersuchungen

2.7. Abschließende Kategorisierung der Fälle S. 40

2.8. Auswertung der gewonnenen Daten

3. ERGEBNISSE $\quad$ S. 42

3.1. Epidemiologische Untersuchungen

$\begin{array}{ll}\text { 3.1.1. Studienteilnahme } & \text { S. } 43\end{array}$

3.1.2. Geschlecht S. 43

3.1.3. Alter S. 43

3.1.4. Verteilung der Todesfälle auf die Wochentage $\quad$ S. 44

3.1.5. Jahreszeitliche Verteilung S. 45

$\begin{array}{ll}\text { 3.1.6. Auffindesituation } & \text { S. } 46\end{array}$

3.1.7. Schwangerschaftsdauer $\quad$ S. 46

3.1.8. Größe und Gewicht zum Zeitpunkt der Geburt
und der Obduktion

3.1.9. Gesundheitszustand vor Todeseintritt $\quad$ S. 49

$\begin{array}{ll}\text { 3.1.10. Geschwister } & \text { S. } 50\end{array}$

3.1.11. Alter der Eltern bei der Geburt des verstorbenen Kindes

S. 51

3.1.12. Soziale Stellung der Familie S. 52

3.1.13. ,Postvitales“ Intervall S. 54

3.1.14. Reanimation durch einen Notarzt $\quad$ S. 57

3.2. Obduktionsbefunde $\quad$ S. 58

3.3. Histologie S. 61

3.4. Bakteriologie S. 64

3.5. Virologie S. 65

3.6. Toxikologie S. 67

3.7. Ethanolbestimmung und Begleitstoffanalyse $\quad$ S. 67

$\begin{array}{ll}\text { 3.7.1. Ethanol S. } 68 & \text { S. } 69\end{array}$

$\begin{array}{ll}\text { 3.7.2. Acetaldehyd } & \text { S. } 69\end{array}$

$\begin{array}{ll}\text { 3.7.3. Methanol S. } 71 & \text { S. } 73\end{array}$

3.7.4. Aceton und Isopropanol $\quad$ S. 73

3.8. Abschließende Kategorisierung S. 75

4. ERGEBNISSE - SIDS IM VERGLEICH ZU NON-SIDS S. 80

$\begin{array}{lll}\text { 4.1. Epidemiologische Untersuchungen } & \text { S. } 80\end{array}$

$\begin{array}{ll}\text { 4.1.1. Geschlecht } & \text { S. } 80\end{array}$

$\begin{array}{lll}\text { 4.1.2. Alter } & \text { S. } 81\end{array}$

4.1.3. Verteilung der Todesfälle auf die Wochentage S. 81

4.1.4. Jahreszeitliche Verteilung $\quad$ S. 82

4.1.5. Auffindesituation $\quad$ S. 84

4.1.6. Schwangerschaftsdauer $\quad$ S. 85

4.1.7. Größe und Gewicht zum Zeitpunkt der Geburt und

$\begin{array}{ll}\text { 4.1.8. Gesundheitszustand vor Todeseintritt } & \text { S. } 88\end{array}$

$\begin{array}{ll}\text { 4.1.9. Geschwister } & \text { S. } 92\end{array}$ 
4.1.10. Alter der Eltern bei der Geburt des verstorbenen Kindes

S. 93

4.1.11. Soziale Stellung der Familie

S. 95

4.1.12. Postvitales Intervall

S. 96

4.1.13. Reanimation durch einen Notarzt

S. 97

4.2. Obduktionsbefunde

S. 99

4.3. Histologie

S. 100

4.4. Bakteriologie

S. 101

4.5. Virologie

S. 102

4.6. Toxikologie

S. 103

4.7. Ethanolbestimmung und Begleitstoffanalyse

S. 103

4.7.1. Ethanol

S. 103

4.7.2. Acetaldehyd

S. 104

4.7.3. Methanol

S. 104

4.7.4. Aceton und Isopropanol

S. 105

4.7.5. Parallele Betrachtung der Begleitstoffanalysen

S. 105

4.8. Die Non-SIDS-Fälle: Zusammenfassung

S. 106

5. TOTENSCHEINANALYSE

S. 109

5.1. Allgemeine Analyse

S. 109

5.2. Todesart laut Todesbescheinigung

S. 110

5.3. Todesart laut Todesbescheinigung bezogen auf SIDS/ Non-SIDS

S. 110

6. DISKUSSION

S. 112

6.1. Diskussion der epidemiologischen Daten

S. 112

6.1.1. Geschlecht

S. 112

6.1.2. Alter

S. 113

6.1.3. Verteilung der Todesfälle auf die Wochentage

S. 114

6.1.4. Jahreszeitliche Verteilung

S. 116

6.1.5. Auffindesituation

S. 117

6.1.6. Schwangerschaftsdauer, Größe und Gewicht zum Zeitpunkt der Geburt und der Obduktion

S. 119

6.1.7. Gesundheitszustand vor Todeseintritt

S. 120

6.1.8. Geschwister

S. 121

6.1.9. Alter der Eltern bei der Geburt des verstorbenen Kindes

S. 122

6.1.10. Soziale Stellung der Familie

S. 122

6.1.11. „Postvitales“ Intervall und Reanimation durch einen Notarzt

6.2. Diskussion der makroskopischen und mikroskopischen Befunde

S. 125

6.2.1. Petechien

S. 125

6.2.2. Respirationstrakt

S. 129

6.2.3. Kongenitale Fehlbildungen

S. 132

6.3. Diskussion der mikrobiologischen Befunde

S. 132 
6.4. Diskussion der toxikologischen Befunde, der Ethanolbestimmung und der Begleitstoffanalyse

6.5. Betrachtung der Non-SIDS-Fälle: Gibt es überhaupt ein SIDS?

6.6. Diskussion der Totenscheinanalyse

6.7. Diskussion des Begriffs und der Definition des „SIDS“

7. SCHLUBFOLGERUNGEN S. 146

$\begin{array}{ll}\text { ZUSAMMENFASSUNG } & \text { S. } 148\end{array}$

$\begin{array}{lr}\text { LITERATURVERZEICHNIS } & \text { S. } 150\end{array}$

$\begin{array}{ll}\text { ANHANG } & \text { S. } 159\end{array}$

$\begin{array}{ll}\text { DANKSAGUNG } & \text { S. } 170\end{array}$

$\begin{array}{ll}\text { LEBENSLAUF } & \text { S. } 171\end{array}$ 


\title{
1. EINLEITUNG UND LITERATURÜBERSICHT
}

\author{
„SIDS ist ein Syndrom, \\ dessen erstes und einziges Symptom der Tod ist!““ \\ [Rognum et al. 1995].
}

1998 verstarben in Deutschland 558 Kinder zwischen dem 7. und 365.Lebenstag unter der Verdachtsdiagnose SIDS [Statistisches Bundesamt 2000]. Der Plötzliche Säuglingstod machte demnach 1998 knapp 30\% der postneonatalen Gesamtmortalität in Deutschland aus.

\subsection{Definition}

Der Plötzliche Säuglings- oder Kindstod wird auch Sudden Infant Death Syndrome (SIDS), Krippentod, Crib Death oder Cot Death genannt.

Nach der ersten offiziellen Definition, die von Beckwith im Jahr 1970 aufgestellt wurde, versteht man unter SIDS den „Plötzlichen Tod jedes Säuglings oder Kleinkindes, der unerwartet eintritt und bei dem eine sorgfältige postmortale Untersuchung keine adäquate Todesursache nachweisen kann“ („The sudden death of an infant or young child which is unexpected by history, and in which a thorough postmortem examination fails to demonstrate an adequate cause of death“) [Beckwith 1970].

Seit 1969 wurden wiederholt Versuche unternommen, andere bzw. bessere Definitionen für den Plötzlichen Säuglingstod zu entwickeln. So wurde 1989 vom National Institute of Child Health and Human Development (NICHD) durch eine Expertengruppe folgende SIDS-Definition empfohlen: „Plötzlicher Tod eines Säuglings unter einem Jahr, der nach einer sorgfältigen Falluntersuchung, einschließlich der Durchführung einer kompletten Autopsie, Untersuchung der Todesumstände und der Betrachtung der klinischen Anamnese ungeklärt bleibt" (The sudden death of an infant under one year of age which remains unexplained after a thorough case investigation, including 
performance of a complete autopsy, examination of the death scene, and review of the clinical history“) [Willinger et al. 1991].

Auf der Dritten Internationalen SIDS-Konferenz in Stavanger in Norwegen (1995) standen schließlich fünf verschiedene, von internationalen SIDS-Forschern entwickelte Definitionen zur Auswahl, unter denen ein Gremium abstimmen sollte. Letztlich fiel die Abstimmung wieder zugunsten der Definition von Beckwith aus dem Jahr 1970 aus, sie besitzt bis heute die größte Akzeptanz unter SIDS-Experten.

\subsection{Sicht des Plötzlichen Säuglingstodes bis zum Ende des 19. Jahrhunderts}

Der Plötzliche Säuglingstod ist ein seit Jahrtausenden bekanntes Phänomen. So wird schon in der Bibel als siebte und schlimmste Plage, die zur Zeit des Auszugs der Israeliten aus Ägypten über den Pharao und sein Land kam, der Tod sämtlicher Erstgeborener erwähnt [Schlaud 1998]. In der Bibel läßt sich noch eine andere Textstelle finden, die von plötzlich und unerwartet verstorbenen Kindern handelt: das „salomonische Urteil“ im Alten Testament (1. Buch Könige 3, 16-28): Zwei Frauen, die im selben Haus wohnen, haben im Abstand von drei Tagen einen Jungen geboren. Eines Nachts starb eines der Kinder, und die Mutter des toten Kindes tauschte den Leichnam heimlich gegen das lebende Kind der anderen Mutter aus. Man sagte, die Frau habe ihren Sohn im Schlaf erdrückt. Diese Erzählung läßt sich am ehesten der SIDS-Theorie des akzidentellen Erstickens zuordnen, einer von drei Theorien über die Ursachen des Plötzlichen Kindstodes, die sich seit Jahrhunderten durch die Literatur ziehen:

- das akzidentelle Ersticken

- das Asthma thymicum

- der Status thymico-lymphaticus

[Kleemann et al. 1997b]. 


\subsubsection{Akzidentelles Ersticken}

Das akzidentelle Ersticken wurde schon im ersten Jahrhundert v.Chr. im alten Ägypten als Ursache für den plötzlichen und unerwarteten Tod bei Kleinkindern angenommen: Hatte die Mutter oder Amme mit dem verstorbenen Kind in einem Bett geschlafen, und es bestand der Verdacht, daß das Kind durch „overlaying“ (Erdrücken des Kindes im Schlaf) getötet wurde, so wurde die Mutter oder Amme für den Tod des Kindes dadurch bestraft, daß sie den Leichnam des Kindes drei Tage und Nächte lang in ihren Armen halten mußte [Guntheroth 1995].

In Deutschland wurde versucht, das plötzliche Versterben von Kleinkindern zu verhindern, indem man im Jahr 1291 Müttern verbot, Kinder unter drei Jahren nachts mit in die elterlichen Betten zu nehmen, was in Dresden von Sudhof öffentlich mittels Plakaten verkündet wurde [Russel-Jones 1985].

Im 17. Jahrhundert wurde in Schweden die Schuld der Eltern, deren Kind plötzlich verstarb, durch einen Richter geprüft. Befand der Richter die Eltern, in der Regel die Mutter, für schuldig, ihr Kind durch „unintentional overlaying“ (unbeabsichtigtes Erdrücken des Kindes im Schlaf) getötet zu haben, so wurde durch die Kirche eine Strafe verhängt. Man stellte die Mutter am Kircheneingang an den Pranger, und erst nach einem öffentlichen Schuldbekenntnis wurde sie wieder in die Kirche und in die Gemeinde aufgenommen [Guntheroth 1995].

Ebenfalls im 17. Jahrhundert wurde in Florenz bereits versucht, Präventionsmaßnahmen für den plötzlichen Tod von Säuglingen einzuführen. Nach einem Bericht von Frank aus dem Jahre 1786 drohte der Mutter oder der Amme die Strafe der Verbannung, sollte sie jemals ein Kind an die Brust oder neben sich ins Bett legen, ohne das sogenannte „Arcuccio“ zu verwenden [Molz 2000]. Das Arcuccio war ein Gestell aus hölzernen und eisernen Bögen, ähnlich dem Dach eines Planwagens, das ein Ersticken verhindern sollte, falls die Mutter während des Schlafes auf ihr Kind rutschen würde [Guntheroth 1995].

In einer Literaturübersicht von Russel-Jones wird unter anderem eine Veröffentlichung Curgenvens aus dem Jahr 1871 aufgeführt, die beschreibt, daß 60\% aller in England obduzierten Säuglinge durch Erdrücken verstorben seien. Besonders häufig geschehe dies samstags in der Nacht, wenn die Mutter eventuell betrunken sei oder besonders 
spät zu Bett gehe, ihrem Kind die Brust gebe und dann einschlafe. Am nächsten Tag sei ihr Kind dann tot. Meistens sei das Kind im Schlaf vom Arm der Mutter gerutscht, sein Gesicht sei unter die Brust der Mutter geraten und das Bettzeug habe zur selben Zeit seinen Kopf bedeckt. Das Kind sei ohne Todeskampf durch das Kohlendioxid seiner eigenen Lungen erstickt. Im Winter passiere dies besonders häufig, da die Mutter sich unvorsichtig das Bettzeug über die Schultern werfe [Russel-Jones 1985].

\subsubsection{Asthma thymicum}

Eine völlig neue Theorie über die Ursache des plötzlichen Todes bei Kleinkindern wurde im Jahre 1830 durch Kopp aufgestellt, der von einem „Asthma thymicum“ als Todesursache ausging. Er berichtet über ein männliches Kind, das plötzlich unter Aufsicht einer Amme verstarb:

„Kurz vor dem Sterben lachte es noch mit der Wärterin und schien munter, als das gewöhnliche Einhalten des Athems auf einmal eintrat, sich aber schnell und im Augenblicke vermehrte, das Gesicht blau wurde, anlief und sogleich der Junge entseelt da lag. Es war das Werk einer Minute. Das Kind erreichte ein Alter von zehn Monaten. Bei der, 22 Stunden nach dem Ableben vorgenommenen, Sektion fand ich Folgendes: Die Zunge etwas stark an der Wurzel und ziemlich lang. Die Luftröhre ohne Fehler. Der Durchgang frei. Die Schilddrüse ausgelaufen und da, wo sie sich mit der Brustdrüse verband, ein Blutextravasat über der Luftröhre. Die Thymus so groß und dick, daß bei Eröffnung der Brusthöhle sie ein anwesender Kunstgenosse für den einen Lungenflügel hielt. Sie hing von ihrer Vereinigung mit der Schilddrüse an bis hinunter zum Zwerchfelle, war bedeutend dick, gegen zwei Zoll breit und lag stark auf der Luftröhre da, wo die Blutunterlaufung sich befand. Innen zeigte sich beim Durchschneiden keine Verhärtung, aber es quoll viel Milchsaft, der die Drüse ganz durchdrang, heraus... Es dürften dem Thymus-Asthma hauptsächlich Kinder männlichen Geschlechts unterworfen seyn..." [Kopp 1830].

Zum erstenmal explizit vom Plötzlichen Säuglingstod als dem ,sudden and unexplained death of children" wurde 1834 von S.W. Fearn gesprochen, und zwar in einem Brief an 
den Herausgeber des Lancet. Fearn berichtete in diesem Schreiben detailliert über die Todesumstände und Obduktionsergebnisse von zwei verstorbenen Kindern, deren Tod er sich nicht erklären konnte, und bat um Hilfe anderer Wissenschaftler, die, wie er hoffte, vielleicht schon ähnliche Fälle gesehen, und möglicherweise eine Erklärung für den Tod der beiden Kinder hätten. Die beiden von ihm obduzierten Kinder hatten zwar einen vergrößerten Thymus, Fearn schrieb diesem Befund aber keine todesursächliche Bedeutung $\mathrm{zu}$, sondern deutete die Thymushyperplasie nur als Begleitbefund bei einigen unerklärlichen, plötzlichen und unerwarteten Todesfällen, sowohl bei Säuglingen, als auch bei Erwachsenen [Fearn 1834].

Auch Friedleben glaubte nicht an einen durch eine Thymushyperplasie verursachten Tod. Er kam 1858, nach Abschluß einer Studie mit anatomischen, physiologischen und experimentellen Untersuchungen, zu dem Ergebnis, daß der Thymus weder in normalem noch in hypertrophem Zustand einen Laryngismus erzeugen könne, und daß es kein Asthma Thymicum gäbe [Kleemann et al. 1997b]. Bestritten wurde die Annahme eines Erstickungstodes durch einen vergrößerten Thymus auch durch Paltauf im Jahr 1890.

\subsubsection{Status thymico-lymphaticus}

Paltauf war der Ansicht, die Todesursache der verstorbenen Kinder sei in der „anormalen lymphatisch-chlorotischen Körperconstitution“ zu suchen, die durch eine Vergrößerung der Tonsillen, Lymphfollikel, ausgebreiteter Lymphdrüsenkomplexe, der Follikel des Zungengrundes, der Milz und das Vorhandensein einer verschieden großen Thymusdrüse charakterisiert sei. Er beobachtete diesen Konstitutionstyp auch bei plötzlich verstorbenen Erwachsenen [Paltauf 1890].

Dieser Symptomenkomplex wurde später von anderen Forschern als Status thymicolymphaticus bezeichnet und zur Grundlage für die Theorie des Thymustodes, die sich bis in die Mitte des 20. Jahrhunderts gehalten hat, obwohl bereits 1931 in England basierend auf 680 Sektionen und ausgedehnten Untersuchungen des Thymus von einem Komitee ausgeschlossen wurde, daß der ,so genannte Status thymico-lymphaticus irgendeine Bedeutung als pathologische Entität hat“" [Young et al. 1931]. Im frühen 20. 
Jahrhundert bis noch in die 20er Jahre wurden bei Kindern prophylaktische Röntgenbestrahlungen des Thymus durchgeführt [Schlaud 1998].

\subsection{Der Plötzliche Säuglingstod ab dem 20. Jahrhundert}

$\mathrm{Zu}$ Beginn des 20. Jahrhunderts verbesserte sich durch soziale Veränderungen der Lebensstandard der Bevölkerung und die meisten Säuglinge hatten ein eigenes Bett zum schlafen. Somit könne es keinen plötzlichen Tod bei Säuglingen durch Erdrücken mehr geben. Da der Plötzliche Säuglingstod aber unverändert weiter beobachtet wurde, ging man zunächst davon aus, daß ein Ersticken durch zu weiche Kopfkissen und Bettwäsche todesursächlich sei [Russel-Jones 1985].

1969 wurde die erste offizielle Definition des Plötzlichen Säuglingstodes von Beckwith publiziert, die bis heute große Akzeptanz findet.

Die World Health Organisation ordnete 1979 dem Plötzlichen Säuglingstod einen ICDCode mit der Nummer $798.0 \mathrm{zu}$.

Welche Bedeutung der Plötzliche Säuglingstod in der medizinischen Forschung hat, und wie intensiv sich die Wissenschaft mit dem Phänomen des Plötzlichen Säuglingstodes auseinandergesetzt hat und weiter auseinandersetzt, zeigt eine von Kenner und Kerbl veröffentlichte Tabelle, mit einem Überblick über die seit dem 19. Jahrhundert verfolgten Theorien zur Ätiologie des Plötzlichen Kindstodes. Nahezu vierzig verschiedene Todesursachen beziehungsweise Auslösemechanismen wurden von zahlreichen Wissenschaftlern untersucht (Tabelle1). 
Tabelle 1: Untersuchte Theorien über die Ursache(n) des SIDS

\begin{tabular}{|c|c|}
\hline Theorie & Wissenschaftler \\
\hline Thymushyperplasie & Grawitz 1888; Paltauf 1889; Feer 1924 \\
\hline Enteropathie & Kolisko 1913 \\
\hline Ersticken & Abramson 1944 \\
\hline Immundefizienz & Spain 1954 \\
\hline Infekt der Luftwege & Schlemer 1956; Althoff 1969 \\
\hline Reflexdysfunktion & Stowens 1957; Kaada 1987 \\
\hline Luftwegsobstruktion & Emery 1958; Shaw 1968; Kahn 1988; Schlüter 1996 \\
\hline Rachitis & Spann 1959 \\
\hline Milchallergie & Parish 1960 \\
\hline Infektionen & Gold 1961; Müller 1961 \\
\hline Streß & Bohrod 1963 \\
\hline Elektrolytentgleisung & Maresch 1964 \\
\hline Anaphylaxie & Gunter 1966 \\
\hline Endokrine Insuffizienz & Geertinger 1966 \\
\hline Tauchreflex & Wolf 1966; French 1972 \\
\hline Bakterielle Infektionen & Hoedt 1966; Johnson 1966; Rognum 1999 \\
\hline Herzrhytmusstörungen & $\begin{array}{l}\text { Church 1967; James 1968; Stramba-Badiale 1995; } \\
\text { Schwartz 1998 }\end{array}$ \\
\hline Wirbelsäulentrauma & Towbin 1967 \\
\hline Hirnödem & Althoff 1969 \\
\hline Laryngospasmus & Bergmann 1969 \\
\hline Virusinfekt & Ray 1970 \\
\hline Schlafapnoen & Steinschneider 1972; Guilleminault 1975; Kelly 1979 \\
\hline Härtegrad des Wassers & Crawford 1973 \\
\hline Autonom. Nervensystem & Salk 1974; Schwartz 1976 \\
\hline Chronische Hypoxie & Naeye 1974 \\
\hline Zerebralschaden & Valdes-Dapena 1976 \\
\hline Glomus caroticum & Geertinger 1976 \\
\hline Diabetes der Mutter & Kirvonen 1976 \\
\hline Atemregulation & Shannon 1977; Kurz 1986 \\
\hline Überwärmung & Nelson 1989; Fleming 1992; \\
\hline Bauchlage & deJonge 1989; Einspieler 1992; Ponsonby 1993 \\
\hline $\mathrm{CO}_{2}$-Rückatmung & Kemp 1991; Bolton 1993; Corbyn 1999 \\
\hline Kollaps kleiner Luftwege & Martinez 1991 \\
\hline Gehirnveränderungen & Molz 1992 \\
\hline Traumerlebnis & Christos 1992 \\
\hline Nikotinbelastung & Milerad 1993 \\
\hline Toxine in Matratzen & Richardson 1994 \\
\hline Zerebrale Perfusionsstörung & Deeg 1998 \\
\hline
\end{tabular}

[modifiziert nach Kenner et al. 2000] 


\subsection{Neue Theorien über die Ursachen des SIDS}

Derzeit geht man davon aus, daß der Plötzliche Säuglingstod ein multifaktorielles Geschehen ist [Sperl 2000; Goldberg et al. 1986].

Die drei im Folgenden dargestellten, aktuellen Theorien über die Ursachen des SIDS sollen ein Hinweis auf die Komplexität des Themas sein: Sie suchen bezüglich der Ätiologie des Plötzlichen Säuglingstodes auf drei völlig verschiedenen Ebenen, und doch kann noch keine dieser Theorien alleine den Plötzlichen Säuglingstod erklären.

\subsubsection{Periodische, zentrale und obstruktive Apnoen}

Eine Überlegung zur Ätiologie des Plötzlichen Kindstodes ist schon seit langem die Apnoe-Theorie, die 1972 von Steinschneider erstmals beschrieben wurde. Er berichtete, daß verlängerte Apnoe-Phasen, die normaler Bestandteil des Schlafes seien, als Teil einer physiologischen Wegstrecke anzusehen seien, die in einen Plötzlichen Säuglingstod münde. Steinschneider hoffte, besonders SIDS-gefährdete Säuglinge an ihrem Schlafverhalten und der Apnoe-Symptomatik identifizieren und sie vor einem SIDS schützen zu können [Steinschneider 1972].

Naeye untermauerte durch histologische Untersuchungen die Theorie, daß zwischen SIDS einerseits und verlängerten Apnoe-Perioden im Schlaf andererseits ein Zusammenhang besteht. Eine chronische alveoläre Hypoventilation sei die wahrscheinlichste Ursache der Hypoxämie, denn die Persistenz braunen Fettgewebes und extramedulläre Hämatopoeseherde seien begleitet von einer Vermehrung glatter Muskelfasern in den kleinen Pulmonalarterien, was ein sensitiver Indikator für eine chronische alveoläre Hypoxie bei fehlenden kardialen Anomalien sei [Neaye 1974]. Inzwischen wurde nachgewiesen, daß es sich bei den von Neaye beobachteten Veränderungen (seine Untersuchungen werden in der Literatur vielfach aufgrund nicht repräsentativen Untersuchungsgutes kritisiert) teilweise um physiologische Entwicklungen handelt, denen keine pathologische Bedeutung zukommt [ValdesDapena 1992]. Kleemann faßte zusammen, daß einige der Befunde eindeutig 
altersabhängig sind, wie die Hämatopoeseherde in der Leber und das persistierende periadrenale braune Fettgewebe [Kleemann 2000a].

Heute geht man davon aus, daß keine Koinzidenz zwischen periodischen Apnoen und SIDS besteht [Kenner et al. 2000]. Nach Willinger ergab sich in einer amerikanischen Studie, daß Apnoen kein spezifisches Kennzeichen für SIDS sind: nur 7\% der Mütter von SIDS-Kindern berichteten von zyanotischen Episoden ihrer Kinder [Willinger et al. 1991].

Anders verhält es sich jedoch möglicherweise mit langen, hypoxämischen zentralen Apnoe-Phasen: Oft wird als Ursache für zentral bedingte Atemregulationsstörungen auf Reifungsprobleme der für die Regulation zuständigen Strukturen im Hirnstamm verwiesen [Wilske 1984].

Bei Säuglingen werden gelegentlich sogenannte ALTE beobachtet. ALTE ( = apparent life threatening event), oder auch ALE (= anscheinend lebensbedrohliches Ereignis), ist definiert als lebensbedrohender Zustand bei Säuglingen, der mit Blässe/ Zyanose und Steifheit oder Schlaffheit einhergeht, bei dem der Tod des Säuglings durch rechtzeitige Reanimation abgewendet werden kann. Es wurde nachgewiesen, daß ein ALTE oft durch eine Verknüpfung von Apnoe und zunehmender Bradykardie charakterisiert ist [Kenner et al. 2000]. Diese Ereignisse werden von anderen Autoren auch „near miss sudden infant death“ oder ,,aborted sudden infant death“ genannt, wie Kurz aufführt. Möglicherweise sind ALTE, und somit auch die Kombination einer Apnoe mit Bradykardie, die Vorstufe eines rechtzeitig abgewendeten SIDS [Kurz 2000]. Jedoch findet sich bei der Mehrzahl der am Plötzlichen Säuglingstod verstorbenen Kinder in der Fremdanamnese kein Hinweis auf ALTE, zyanotische Episoden und Apnoen [Willinger et al. 1991].

Obstruktive Apnoen dagegen sind unter den Apnoe-Formen diejenigen, die am wahrscheinlichsten einen SIDS verursachen können [Willinger et al. 1991], nicht zuletzt wegen der noch zarten und instabilen Luftwege eines Säuglings und der Tatsache, daß Säuglinge obligate Nasenatmer sind [Kenner et al. 2000].

Eine gestörte oder verzögerte Reifung der für die Atemregulation zuständigen Strukturen alleine wird den Plötzlichen Säuglingstod nicht erklären können. Man hätte keine Erklärung dafür, daß im ersten Lebensmonat kaum SIDS-Fälle auftreten, jedoch im zweiten bis vierten Monat besonders viele. Ebenso ließe sich nicht die 
unterschiedliche Verteilung auf die Geschlechter erklären, wenn auch unter Erwachsenen Männer häufiger an Schlafapnoen leiden als Frauen [Wilske 1984]. Wie Wilske zusammenfaßt, ist aus experimentellen Untersuchungen bekannt, daß eine Hypoxie des Myokards (wie sie aus einer auf Schlafapnoen beruhenden Sauerstoffuntersättigung des Blutes resultieren kann) über einen atrioventrikulären Überleitungsblock zu Reizleitungsstörungen und lebensbedrohlichen Arrhythmien führen kann. Auch während Apnoe-Phasen zu beobachtende Bradykardien sind zum Teil durch einen hypoxämischen Herzmuskel erklärbar [Wilske 1984].

Nach Willinger geht eine spontane Erholung von einer andauernden Apnoe normalerweise mit einem Aufschrecken aus dem Schlaf und einem hypoxisch bedingten Schnappen nach Luft einher. Bei SIDS-Fällen könnte dieser funktionelle Mechanismus mangelhaft ausgebildet sein, und so in einem Plötzlichen Säuglingstod enden [Willinger et al. 1991].

\subsubsection{Das QT-Syndrom}

Eine weitere mögliche Ursache für den Plötzlichen Säuglingstod ist das QT-Syndrom, welches auch Pseudohypokaliämiesyndrom genannt wird. Von diesem Syndrom gibt es unter anderem zwei erbliche Formen: das Jervell-Lange-Nielse-Syndrom und das Romano-Ward-Syndrom.

Obwohl in den 80er Jahren der prolongierten QT-Dauer keine wesentliche Bedeutung im Zusammenhang mit dem Plötzlichen Säuglingstod mehr beigemessen wurde [Wilske 1984], so wurde in jüngster Zeit die Diskussion über die Bedeutung des QT-Syndroms für die Ätiologie des SIDS wieder aufgenommen. Nach Untersuchungen von Schwartz wiesen 12 von 24 SIDS-Fällen eine Herzrhythmusstörung im Sinne einer prolongierten QT-Phase im EKG auf [Schwartz et al. 1998]. Inwieweit diese Form einer Herzrhythmusstörung in Verbindung mit dem Plötzlichen Säuglingstod steht, ist noch nicht geklärt, und wird zurzeit von mehreren Forschergruppen untersucht [Rognum 2000b]. 


\subsubsection{Zytomegalievirus-Infektion}

Im Rahmen verschiedener Studien wurden verstorbene Säuglinge hinsichtlich einer Zytomegalievirus (ZMV)-Infektion untersucht. Valdes-Dapena faßt die Untersuchungsergebnisse verschiedener Autoren bezüglich ZMV-Infektionen bei SIDS-Fällen in einer Literaturübersicht zusammen:

1986 wurden von Variend und Pearse bei vier untersuchten, plötzlich und unerwartet verstorbenen Kindern, zytomegale Einschlußkörperchen in nicht-neuronalen Geweben nachgewiesen. Huff und Carpenter wiesen unter 58 SIDS-Fällen in 12\% ZMV-Zellen nach, unter 401 Todesfällen aus geklärter Ursache waren es nur 1\%. Variend veröffentlichte 1990 Ergebnisse einer weiteren Studie mit 951 Kindern. Bei 24 der Kinder konnten zytomegale Einschlüsse in der Glandula parotis nachgewiesen werden. Püschel et al. untersuchten retrospektiv 180 SIDS-Fälle aus Berlin und Hamburg auf ZMV. Sie fertigten nicht nur HE-Färbungen an, wie die anderen Untersucher, sondern wandten immunhistochemische Methoden an. Bei 10\% der Berliner und bei 7\% der Hamburger SIDS-Fälle konnten so das ZMV nachgewiesen werden [Valdes-Dapena 1991].

In einer Untersuchung mittels in-situ Hybridisierung des Aachener Instituts für Rechtsmedizin wurden bei 5\% der untersuchten 122 SIDS-Fälle ZMV-Infektionen festgestellt [Cremer et al. 1990]. Diese nachweisbare Prävalenz von ZMV-Infektionen unter SIDS-Fällen führte $\mathrm{zu}$ der Frage, ob möglicherweise unentdeckte intrauterine ZMV-Infektionen, vielleicht durch Schädigungen des Hirnstammes, indirekt für einen Teil der SIDS-Fälle verantwortlich sind [Valdes-Dapena 1991].

\subsection{Epidemiologie und Risikofaktoren}

Von Land zu Land herrschen teils große, teils keinerlei Unterschiede im Hinblick auf Einzelaspekte der Epidemiologie des SIDS. So lassen sich beispielsweise große länderspezifische Unterschiede bezüglich der Inzidenz des Plötzlichen Säuglingstodes und dessen Anteil an der Gesamtsäuglingssterblichkeit des entsprechenden Landes 
feststellen. Hingegen gibt es kaum oder keine Unterschiede in Bezug auf die Geschlechtsverteilung oder saisonale Häufungen.

Dennoch wurden in der Vergangenheit verschiedene Risikofaktoren entdeckt, die beim Auftreten des Plötzlichen Kindstodes eine maßgebliche Rolle spielen. Einleitend seien hier männliches Geschlecht, Alter zwischen einem und sechs Monaten, bestimmte ethnische Zugehörigkeiten, Schlafen in Bauchlage, niedriges Geburtsgewicht, kurze Schwangerschaftsdauer, Rauchen der Mutter während der Schwangerschaft, niedriger Bildungsgrad/ sozialer Status der Mutter oder beider Eltern, Überwärmung, ledige Mütter, junge Mütter und Nichtstillen genannt.

Im Folgenden werden die epidemiologischen Fakten und die wichtigsten Risikofaktoren genauer dargestellt.

\subsubsection{SIDS-Inzidenz und Anteil an der postneonatalen Gesamtmortalität}

1998 verstarben in Deutschland 1990 Kinder zwischen dem siebenten und 365. Lebenstag. In 558 der Fällen wurde die Diagnose „SIDS“ gestellt [Statistisches Bundesamt 2000], wobei aber nicht alle diese Kinder obduziert wurden und somit per definitionem eine sichere SIDS-Diagnose überhaupt nicht zu stellen ist. Der Plötzliche Säuglingstod macht demnach knapp 30\% der postneonatalen Gesamtmortalität aus (Abb. 1). Ein solch hoher Anteil wird im untersuchten Zeitraum nur noch von Schottland und Neuseeland erreicht. In der Literatur wird die SIDS-Mortalität in Deutschland sogar mit einem Anteil von bis $\mathrm{zu} 40 \%$ an der postneonatalen Gesamtmortalität angegeben [Jorch 2000]. Auch in Bezug auf die SIDS-Inzidenz nimmt Deutschland nach Neuseeland eine Spitzenstellung ein (Abb. 2). Die folgenden zwei Diagramme geben einen Überblick über den SIDS-Anteil an der postneonatalen Gesamtmortalität und die weltweite SIDS-Inzidenz, basierend auf den aktuellsten Daten der World Health Organisation, das heißt je nach Land aus den Jahren 1995 - 1998 [World Health Organisation 2000]. 
Abb. 1: SIDS-Anteil an der postneonatalen Gesamtmortalität (1995 - 1998), WHO

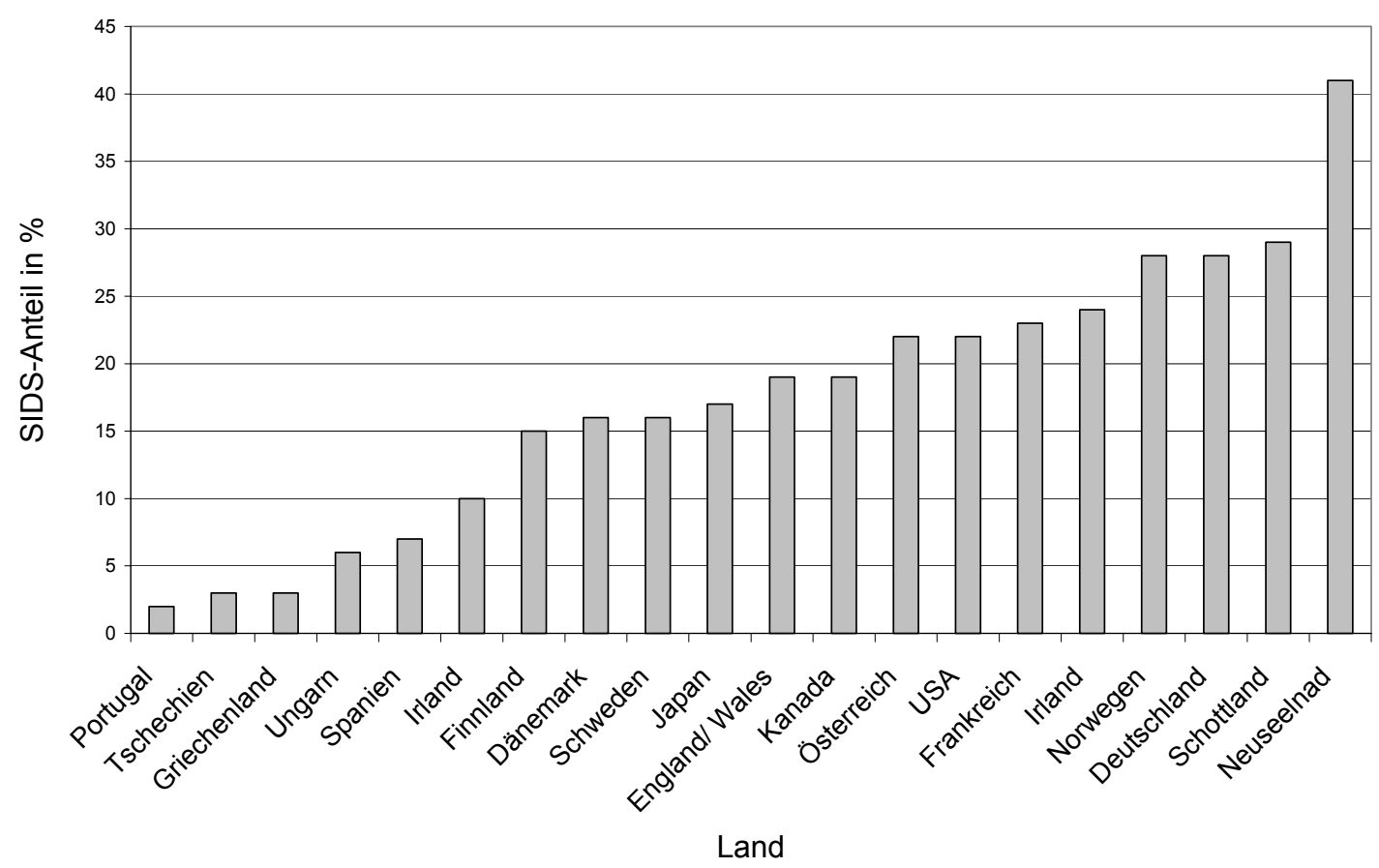

Abb. 2: SIDS-Inzidenz zwischen 1995 und 1998 laut WHO

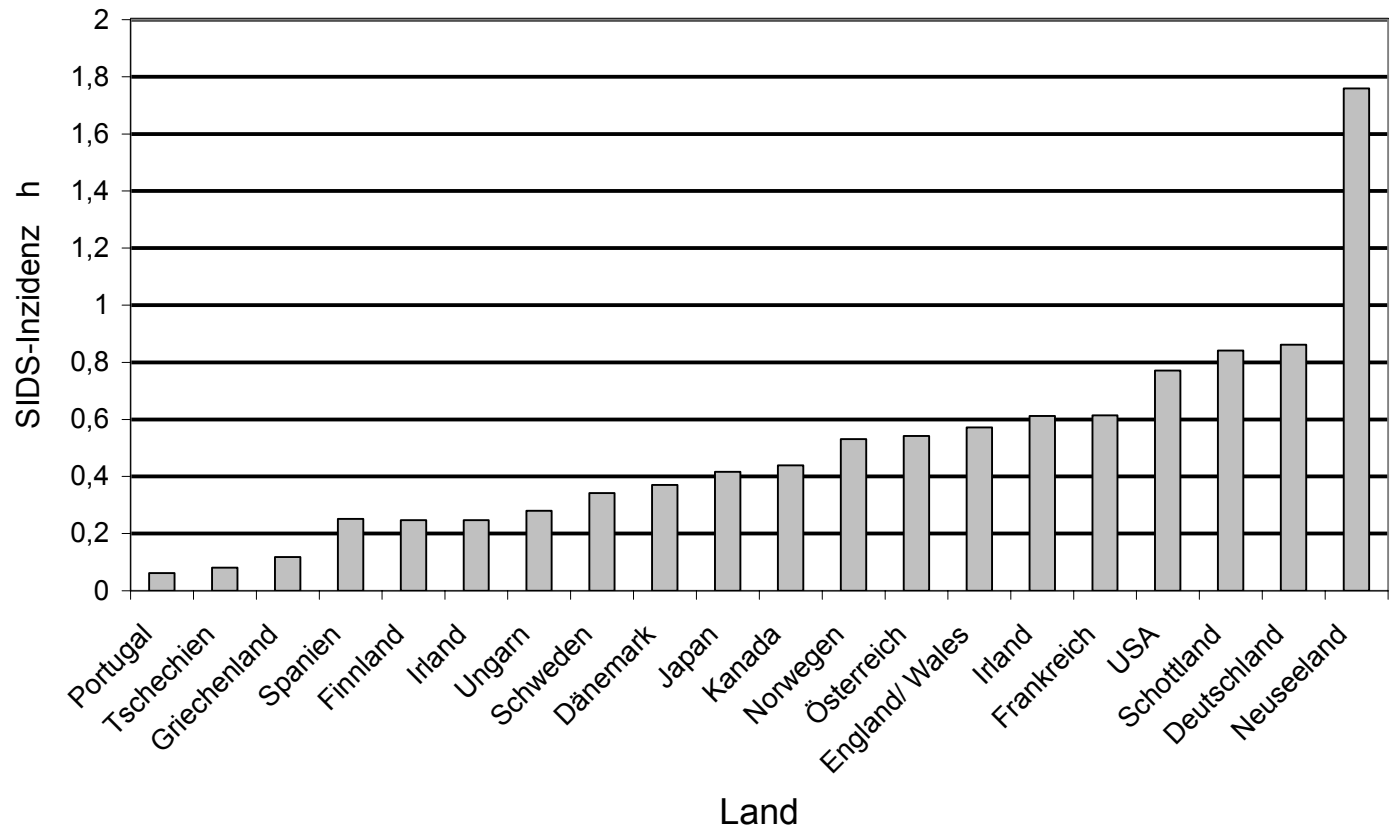


Die Spannbreite der SIDS-Inzidenz lag 1987 noch zwischen 0,1 und 5,9 pro 1000 Lebendgeborenen [Jorch 2000], in den letzten Jahren war sie stark rückläufig und ist mittlerweile zwischen 0,062 (Portugal) und 0,836 (Deutschland) beziehungsweise 1,759 (Neuseeland) anzusiedeln.

In den letzten zehn Jahren kam es in vielen Ländern zu einem starken Rückgang der SIDS-Inzidenz, so auch in Deutschland, wobei die Zahlen zur Inzidenz sehr hoch erscheinen und eventuell auch an Erhebungsfehler denken lassen, auch da die Angaben des statistischen Bundesamtes (Tabelle 2) deutlich von den Angaben der WHO (Abb. 2) abweichen.

Tabelle 2: SIDS-Anteil an der postneonatalen Säuglingssterblichkeit und SIDS-Inzidenz der letzen Jahre in Deutschland

\begin{tabular}{|c|c|c|}
\hline Jahr & $\begin{array}{c}\text { SIDS-Anteil } \\
\text { an der postneonatalen } \\
\text { Säuglingssterblichkeit }\end{array}$ & $\begin{array}{c}\text { Inzidenz } \\
\text { (SIDS-Fälle pro 1000 } \\
\text { Lebendgeburten) }\end{array}$ \\
\hline 1988 & $33.9 \%$ & 3.01 \\
\hline 1990 & $37.5 \%$ & 3.26 \\
\hline 1992 & $30.3 \%$ & 2.21 \\
\hline 1994 & $28,6 \%$ & 1.83 \\
\hline 1996 & $30.6 \%$ & 1.61 \\
\hline 1998 & $28.0 \%$ & 1.42 \\
\hline
\end{tabular}

[zusammengestellt aus den Statistischen Jahrbüchern des Statistischen Bundesamtes 1990 - 2000]

Nach einer vorläufigen Inzidenz-Schätzung für das Jahr 1999 der Universität Münster im Rahmen der dieser Arbeit zugrundeliegenden Studie (siehe Kapitel 1.7.) lag diese nur noch bei etwa 0,81 pro 1000 lebend Geborenen [Westfälische Wilhelms-Universität Münster 2000].

Zum Teil ist in den einzelnen Ländern eine zeitliche Zuordnung des InzidenzRückgangs zu "Back to sleep"-Kampagnen möglich, die Anfang der 90er Jahre durchgeführt wurden, jedoch nicht immer. Nach Jorch lassen sich seit 1987 in den einzelnen Ländern verschiedene Jahreszahlen mit den jeweils stärksten Rückgängen der SIDS-Inzidenz beobachten (Tabelle 3 ): 
Tabelle 3: Rückgang der SIDS-Inzidenz in Europa während der letzten 15 Jahre

\begin{tabular}{|c|c|}
\hline Land & $\begin{array}{c}\text { Jahr mit dem stärksten SIDS- } \\
\text { Rückgang }\end{array}$ \\
\hline Niederlande & 1988 \\
\hline Norwegen & 1990 \\
\hline Dänemark & 1992 \\
\hline Deutschland & 1992 \\
\hline Australien & 1993 \\
\hline England/Wales & $1989 / 1992$ \\
\hline Irland & $1989 / 1993$ \\
\hline Österreich & $1989 / 1994$ \\
\hline USA & $1992 / 1994$ \\
\hline Frankreich & $1992 / 1995$ \\
\hline
\end{tabular}

[modifiziert nach Jorch 2000]

\subsubsection{Tages- und jahreszeitliche Verteilung}

In einer Studie aus Großbritannien zeigte sich, daß ein Großteil der am Plötzlichen Säuglingstod verstorbenen Kinder, nämlich 83\%, während des nächtlichen Schlafes verstarben. Ferner hob sich kein Wochentag durch besonders viele oder wenige Todesfälle hervor [Leach et al. 1999].

Im Gegensatz dazu wiesen Williams et al. in Neuseeland eine signifikante Häufung der SIDS-Fälle an Wochenenden nach, wobei der Sonntag so häufig vertreten war, wie sonst kein anderer Wochentag. Unter neuseeländischen Maoris und Kindern unverheirateter Mütter trat besonders häufig ein SIDS an einem Sonntag auf [Williams et al. 1997]. Auch in Deutschland (Niedersachsen) starben zwischen 1985 und 1989 samstags und sonntags mehr Kinder als an anderen Wochentagen [Lohmann 1996].

Wilske fand eine gehäufte SIDS-Sterblichkeit in den Frühjahrs- und Herbstmonaten [Wilske 1984]. Auch in einer amerikanischen Studie an 190 SIDS-Fällen ergab sich eine Häufung der SIDS-Fälle im späten Herbst und Winter [Standfast et al. 1983]. Lohmann stellte fest, daß in Niedersachsen zwischen 1985 und 1989 im Oktober bis Januar überdurchschnittlich viele, in den Monaten Februar bis September unterdurchschnittlich viele Kinder am SIDS verstarben [Lohmann 1996]. In einer Studie aus Großbritannien zeigte sich wiederum eine Abschwächung der 
Sterblichkeitsspitze in den Wintermonaten: nur knapp ein Viertel der SIDS-Fälle ereignete sich in den drei Wintermonaten [Leach 1999].

\subsubsection{Geschlechtsverteilung}

In sämtlichen epidemiologischen Studien wurde festgestellt, daß Jungen unter den SIDS-Fällen überrepräsentiert sind, und zwar mit einem Anteil von etwa $52-55 \%$. Nach Wilske ergibt sich mit 60\% sogar eine Jungenübersterblichkeit gegenüber den Mädchen. Bei Aufschlüsselung in Todesfälle des ersten und zweiten Lebenshalbjahres stellte Wilske fest, daß im ersten Lebenshalbjahr mehr Jungen (70\%) als Mädchen (30\%) verstarben, es im zweiten Lebenshalbjahr jedoch zu einer Umkehr der Verhältnisse mit einer Mädchenübersterblichkeit (56\%) kam [Wilske 1984].

Wie Guntheroth berichtet, wurde in diversen Studien nachgewiesen, daß bei Indianern Nordamerikas und bei Einwohnern Alaskas diese Überrepräsentation des männlichen Geschlechts nicht nachweisbar ist, was durch andere Studien wiederum nicht bestätigt werden konnte [Guntheroth 1995].

Wilske berichtet weiter, daß die Jungenübersterblichkeit beim Plötzlichen Säuglingstod stärker ausgeprägt sei als bei anderen Todesursachen [Wilske 1984]. Jorch jedoch kommt zu dem Schluß, daß ein Überwiegen des männlichen Geschlechts bei einem Anteil von 52 - 56\% kein typisches Kriterium für den Plötzlichen Säuglingstod sei, denn auch bei den meisten anderen Todesursachen im Kindesalter zeige sich oft eine Bevorzugung des männlichen Geschlechts [Jorch 2000].

\subsubsection{Altersverteilung}

Die Angaben in der Literatur über das typische Alter der verstorbenen Säuglinge variieren nur geringfügig.

Laut Jorch versterben am häufigsten Kinder im Alter von zwei bis fünf Monaten am SIDS. In den ersten beiden Lebenswochen und jenseits des 10. Lebensmonates ist ein Plötzlicher Säuglingstod selten [Jorch 2000]. 
Guntheroth beschreibt, daß vor allem Kinder zwischen einem und sechs Monaten am SIDS versterben, und stellt einen Altersgipfel im zweiten und dritten Lebensmonat fest [Guntheroth 1995].

Andere Autoren bestätigen eine Häufung der Säuglingstod-Fälle im zweiten und dritten [Wilske 1984; Carpenter 1983; Standfast et al. 1983] bzw. im zweiten bis vierten Lebensmonat [Penning et al. 1996].

Wiske berichtet, daß die im ersten Lebensjahr beobachtete Sterblichkeitsquote nach Vollendung des ersten Lebensjahres abrupt auf ein Minimum zurückgeht und erst wieder von 60- bis 65jährigen erreicht wird [Wilske 1984].

Die Altersverteilung bei posttraumatischen oder krankheitsbedingten Todesfällen unterscheidet sich signifikant von der Altersverteilung beim Plötzlichen Säuglingstod [Kraus 1983; Wilske 1984; Goldberg 1986]. Goldberg verglich die "age at death curve" von SIDS-Fällen mit denen von 21 anderen Todesursachen und kam zu dem Schluß, daß sich die SIDS-Kurve signifikant von allen anderen unterscheidet. Ferner sei das Alter bei Todeseintritt unabhängig von Risikofaktoren wie beispielsweise ethnischer Zugehörigkeit, Geburtsgewicht, Alter der Mutter und Geschlecht [Goldberg 1986].

Die folgende, nach Emery modifizierte Abbildung zeigt die Altersverteilung bei plötzlich unerwartet verstorbenen Kindern (SIDS) im Vergleich zur Altersverteilung bei im Krankenhaus verstorbenen Kindern. Diese leider schon älteren Daten wurden 1976 im Rahmen einer multizentrischen Studie in Großbritannien erhoben, die etwa 1000 Kindstod-Fälle und entsprechende Kontrollen umfaßte (Abb.3). 


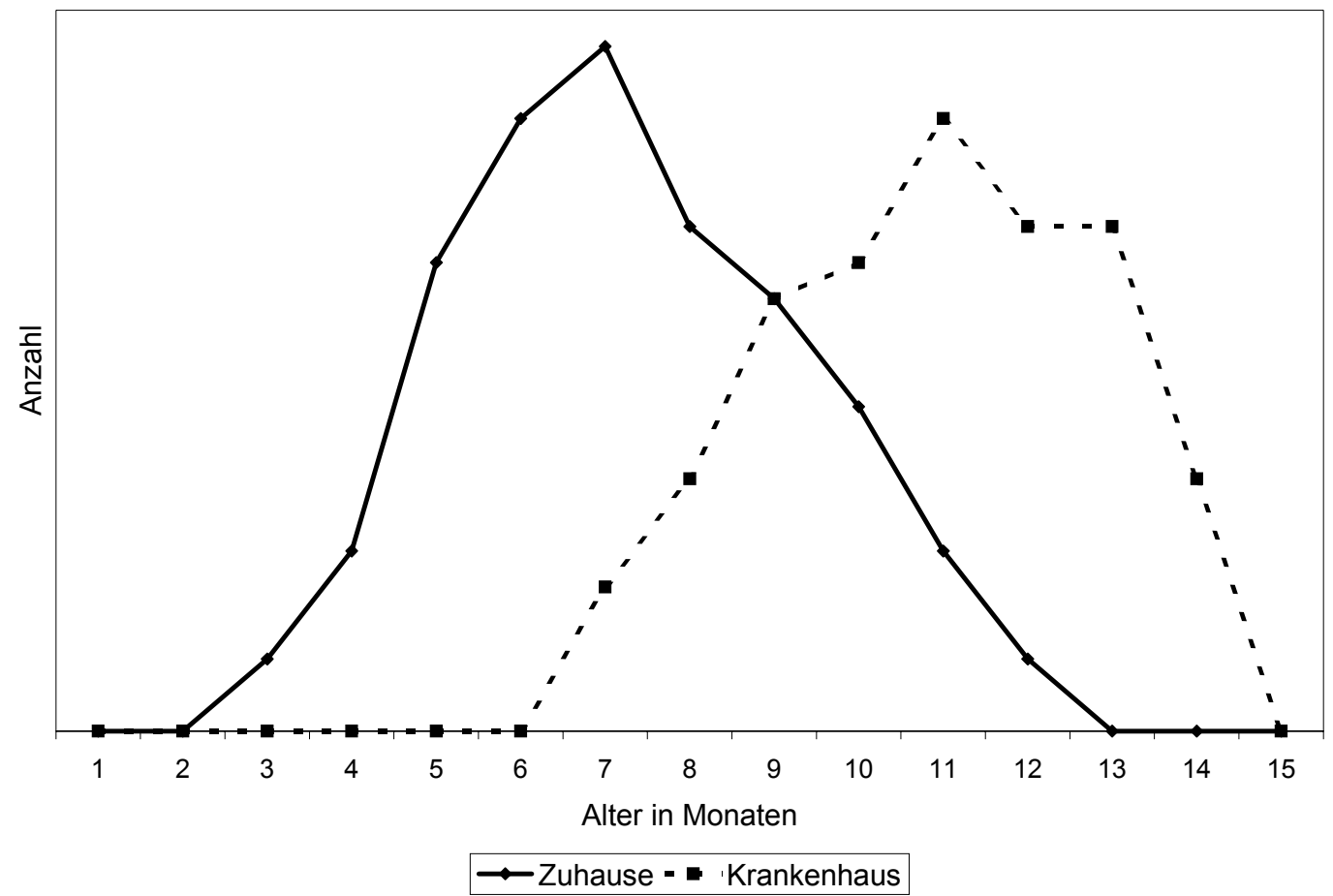

[Emery 1983]

\subsubsection{Auffindesituation, Bauchlage und "Back to sleep"-Kampagnen}

"Die Kinder werden meist nach dem Schlaf, häufig in Bauchlage tot aufgefunden. Teilweise sind sie -offenbar agonal- mit dem Kopf unter eine Decke gekrochen, ohne daß (im Kontrast zu den häufigen Selbstvorwürfen der Eltern) ein Erstickungstod in Frage käme. Vereinzelt findet sich agonales Erbrechen oder etwas abgeronnenes, wäßriges Sekret um Mund und Nase. Nicht ganz selten sind die Kinder völlig durchgeschwitzt - Temperaturen bis $40^{\circ} \mathrm{C}$ wurden unmittelbar postmortal beobachtet" [Penning et al. 1996].

Während noch 1984 die Frage, ob die Bauch- oder die Rückenlage den Eintritt eines Plötzlichen Säuglingstodes begünstigen könnten, als irrelevant eingeschätzt wurde [Wilske 1984], so steht inzwischen fest, daß der Zusammenhang zwischen Bauchlage und gehäuftem Auftreten des SIDS kein Artefakt ist, sondern die Bauchlage in der Ätiologie des Plötzlichen Säuglingstodes eine bedeutende Rolle spielt [Schlaud 1998]. Kleemann kommt nach Auswertung einer Literaturübersicht zu dem Schluß, daß es sich 
bei dem beobachteten Zusammenhang zwischen Bauchlage und Plötzlichem Kindstod nicht um einen Zufall handeln kann, sondern daß vielmehr von einem relativen Risiko von 2,8 bis 6,3 auszugehen ist. Das heißt, daß Kinder, die in Bauchlage schlafen, das etwa drei- bis sechsfache Risiko tragen, an einem Plötzlichen Säuglingstod zu versterben [Kleemann et al. 1995a].

In Norwegen verglich ein Forscherteam die Verwendung der Bauchlage und die SIDSInzidenz vor und nach "Back to sleep"-Kampagnen (öffentliche Kampagnen, die die Rückenlage als sicherste Lage für schlafende Säuglinge propagieren). Vor dieser Kampagne, in den Jahren 1987 - 1989, wurden 64\% der beobachteten Säuglinge von den Eltern zum Schlafen auf den Bauch gelegt. Nach der Kampagne, im Zeitraum zwischen 1990 und 1992, waren es nur noch 8\%. Zeitgleich sank die SIDS-Inzidenz von 3,5 auf 1,6 pro 1000 Lebendgeburten [Markestad et al. 1995].

Es gibt verschiedene Hypothesen darüber, warum die Bauchlage einen Risikofaktor für den Plötzlichen Säuglingstod darstellt, diskutiert werden unter anderem ein begünstigtes bakterielles Wachtum in der Nasenflora [Bell 1996], erschwerte Regulation der Körpertemperatur [Rognum 2000a], vasovagale Reaktionen im Sinne eines Tauchreflexes, Kohlendioxid-Rückatmung, eine veränderte Gehirndurchblutung, positionsabhängige Asphyxie und reduzierte Aufwachreaktionen [Kenner et al. 2000; Bell et al. 1996]. Keine der Theorien konnte sich jedoch bisher als Erklärung für den Todeseintritt durchsetzen.

\subsubsection{Postnatale Entwicklung der SIDS-Fälle}

Kinder mit einem Geburtsgewicht von weniger als zwei Kilogramm hatten, nach einer Untersuchung von Beal, im Vergleich zu bei der Geburt normalgewichtigen Kindern, eine auf das Doppelte erhöhte SIDS-Inzidenzrate [Beal 1983].

Nach Molz neigen die SIDS-Fälle vor dem Tod zu einer verzögerten kindlichen Entwicklung: Die Körpergewichte der SIDS-Fälle lagen bei der Geburt über der 50. Perzentile, bei der Obduktion hingegen insgesamt unter der 50. Perzentile, so daß die 
SIDS-Kinder offensichtlich zu einer verzögerten Entwicklung tendieren. Dasselbe gilt auch für die Körperlänge und den Kopfumfang [Molz 1998].

Auch nach Valdes-Dapena hat eine Gruppe von Epidemiologen in Washington festgestellt, daß die SIDS-Fälle zum Zeitpunkt der Geburt eine geringere Durchschnittsgröße hatten als die Kontrollfälle [Valdes-Dapena 1991].

\subsubsection{Ethnische Abstammung}

Zum ersten Mal von einem ethnisch bedingten SIDS-Risiko berichtet wurde von Adelson und Kinney im Jahr 1956. Sie stellten fest, daß in ihrem Untersuchungsgut 16\% der lebend geborenen Kinder Schwarze waren, diese jedoch unter den SIDS-Fällen mit einem Anteil von 27\% überrepräsentiert waren [Adelson et al. 1956].

Auch nach Valdes-Dapena gab es 1979 in den USA unterschiedliche SIDS-Inzidenzen bei weißen (Inzidenz 1,1 pro 1000) und schwarzen Kindern (Inzidenz 3,65 pro 1000) [Valdes-Dapena et al. 1993].

In Washington State wurde von Irwin für die Ureinwohner Amerikas ein dreifach erhöhtes SIDS-Risiko nachgewiesen: Dieses hohe Risiko beruhe allerdings vermutlich auf der hohen Prävalenz von SIDS-Risikofaktoren von Seiten der Mütter der verstorbenen Kinder, und nicht etwa auf genetischen Faktoren. Diese Risikofaktoren wiederum seien eng mit dem sozioökonomischen Status dieser Frauen verknüpft. Vereinfacht könne man sagen, ein niedriger sozialer Status bedinge bei den Ureinwohnern Amerikas ein erhöhtes SIDS-Risiko [Irwin et al. 1992].

Dies läßt sich auch bei anderen Volksgruppen beobachten, zum Beispiel bei den Ureinwohnern Australiens: In Australien war zwischen 1980 und 1990 das SIDS-Risiko von Ureinwohnern (Aborigines) 3,86 mal höher als das von Nicht-Ureinwohnern. Auch hier waren die Risikofaktoren für den Plötzlichen Säuglingstod unter den Ureinwohnern weiter verbreitet als unter den Nicht-Ureinwohnern [Alessandri et al. 1996].

In einer weiteren amerikanischen Studie wurde beobachtet, daß die SIDS-Inzidenz für Schwarze bei 5,1 pro 1000 Lebendgeburten, für Weiße bei 1,3 und für Hispanos bei nur 1,2 lag. In jeder dieser ethnischen Gruppen stieg die SIDS-Inzidenz mit abnehmendem Geburtsgewicht kontinuierlich an. So lag in der Gruppe der Kinder mit einem 
Geburtsgewicht unter 1500 Gramm die SIDS-Inzidenz für Schwarze bei 16,4 pro 1000 Lebendgeburten, bei Weißen bei 5,5 und bei Hispanos, obwohl sie sozioökonomisch unterprivilegiert waren, bei nur 3,9 pro 1000. Dies impliziert, daß nicht nur das Einkommen und die Bildung eine Rolle beim Plötzlichen Kindstod spielen, sondern auch kulturelle Faktoren [Valdes-Dapena 1991].

\subsubsection{Weitere Risikofaktoren}

Außer den bereits zuvor dargestellten möglichen Risikofaktoren für einen Plötzlichen Säuglingstod werden in der Literatur noch weitere SIDS-Risikofaktoren aufgeführt, von denen die wichtigsten hier kurz aufgeführt werden sollen:

- Geringes Alter der Mutter bei der Geburt des verstorbenen Kindes:

Lohmann beobachtete, daß in Oregon/ USA zwischen 1985 und 1989 die Mütter der an einem SIDS verstorbenen Kinder ein durchschnittliches Alter von 21,4 Jahren hatten. Mehr als die Hälfte der Mütter von verstorbenen SIDS-Fällen waren höchstens 20 Jahre alt, und nur $20 \%$ der Mütter waren über 24. Verglichen mit den Müttern von Kontrollfällen waren die Mütter von SIDS-Fällen signifikant jünger [Lohmann 1996]. Auch andere Autoren berichten von einer Überrepräsentation junger Mütter unter den SIDS- Fällen [Valdes-Dapena et al. 1993; Willinger et al. 1991].

- Niedriges Bildungsniveau der Mutter bzw. der Eltern:

In Oregon/ USA betrug nach einer Studie die durchschnittliche Ausbildungsdauer von SIDS-Müttern 11,7 Jahre. Eine Ausbildung von mehr als zwölf Jahren absolvierten etwa $21 \%$ der SIDS-Mütter. Die Mütter der Kontrollfälle, also der nicht am SIDS verstorbenen Kinder, befanden sich durchschnittlich 12,9 Jahre in Ausbildung, und etwa $42 \%$ von ihnen hatten eine Ausbildungsdauer von mehr als zwölf Jahren [Lohmann 1996]. 
- Rauchen während der Schwangerschaft:

Das SIDS-Risiko bei Kindern von Raucherinnen ist um das 2,5fache erhöht. Mit zunehmendem durchschnittlichem Zigarettenkonsum pro Tag während der Schwangerschaft konnte auch eine konsistente Zunahme des relativen Risikos für das SIDS beobachtet werden [Schlaud 1998]. So berichtet Rath, daß ein Zigarettenkonsum von fünf bis neun Zigaretten pro Tag das SIDS-Risiko auf etwa das Vierfache erhöht. Bei einem Konsum von zehn und mehr Zigaretten pro Tag erhöht sich das Risiko sogar auf das Zwölffache [Rath 1998]. In anderen Untersuchungen weichen die Zahlenwerte gering ab: Valdes-Dapena berichtet über ein zweifach erhöhtes SIDS-Risiko bei einem Zigarettenkonsum von bis zu neun Zigaretten während der Schwangerschaft. Ab zehn Zigaretten pro Tag sei das Risiko auf das Dreifache erhöht [Valdes-Dapena 1991].

Aus einer Berliner Studie geht hervor, daß 55\% der SIDS-Mütter während der Schwangerschaft rauchten, in der Kontrollgruppe waren es nur 8\% [Rath 1998]. Auch in der Westfälischen Kindstodstudie wurde in den Jahren 1990 bis 1993 mütterliches Rauchen während der Schwangerschaft als dominierender, und darüberhinaus dosisabhängiger, Risikofaktor ermittelt [Jorch et al. 1995]. Viele andere Untersuchungen bestätigen Rauchen als vermeidbaren Risikofaktor bei der Entstehung des SIDS [Naeye 1990].

- Soziale Situation:

Von Lohmann ausgewertete Daten einer amerikanischen Studie ergaben, daß sich die SIDS-Familien in soziodemographischer Hinsicht signifikant von den KontrollFamilien unterschieden. So waren in der SIDS-Gruppe 67\% der Mütter verheiratet. In der Kontrollgruppe hingegen waren es 84\%. Die Anzahl der ledigen Mütter in der SIDS-Gruppe war somit signifikant höher als in der Kontrollgruppe.

In der SIDS-Gruppe war in 26\% der Vater des Kindes nicht bekannt oder wurde von der Mutter zumindest nicht genannt. Bei der Kontrollgruppe war dies nur in 10\% der Fall. In der Gruppe der Plötzlichen Kindstodfälle waren weniger Familien mit nur einem oder zwei Kindern; vermehrt kamen die verstorbenen SIDS-Kinder aus Familien mit drei oder vier Kindern [Lohmann 1996]. 
- Wiederholungsrisiko:

Eine Familie, die ein Kind durch den Plötzlichen Säuglingstod verloren hat, soll ein sechsfach erhöhtes Risiko haben, auch ein zweites Kind durch das SIDS verlieren [Guntheroth et al. 1990].

Nach Penning sollte man allerdings solche Statistiken mit Zurückhaltung betrachten, da es Untersuchungen gibt, in denen die eigenen Eltern bei hospitalisierten, apnoegefährdeten Kindern bei mechanischen Erstickungsversuchen am Kind gefilmt wurden; in der Familienanamnese fanden sich bis zu drei angebliche SIDS-Fälle, bei denen trotz Obduktion keine andere Todesursache festgestellt wurde [Penning et al. 1996].

\subsection{Obduktion}

Die Diagnose "SIDS", die in Deutschland in 30\% der Todesfälle bei Säuglingen gestellt wird, ist per definitionem nur nach erfolgter Obduktion möglich. Trotzdem läßt sich in vielen Leichenschauscheinen nachlesen, daß bereits der die Leichenschau durchführende Arzt die Diagnose "Plötzlicher Säuglingstod" gestellt hat. Auch in anderen Ländern wurde und wird die Diagnose "SIDS" im Totenschein oft ohne Durchführung einer Obduktion verwendet. Daher hat schon 1989 ein Expertenkomitee in den USA angeregt, daß die Diagnose eines Plötzlichen Säuglingstodes nur nach Erhebung einer Familienanamnese, Untersuchung der Todesumstände und einer Obduktion erfolgen dürfe [Willinger et al. 1991].

Trotz aller Argumente, die für eine Obduktion sprechen, wurden zwischen 1985 und 1989 in Deutschland, je nach Region, durchschnittlich nur zwischen 51\% und 59\% der angeblichen Plötzlichen Kindstodfälle obduziert, mit allerdings erheblichen Schwankungen zwischen den einzelnen Bundesländern: 
Tabelle 4: Unter der ICD-Ziffer 798.0 im Leichenschauschein erfaßte versus durch Obduktion von Säuglingen bestätigte SIDS-Fälle in der BRD nach Bundesländern für die Jahre 1985-1989

\begin{tabular}{|l|c|c|c|}
\hline \multirow{2}{*}{ Bundesland } & ICD 798.0 & \multicolumn{2}{c|}{ Obduktionen } \\
\cline { 2 - 4 } & $\mathbf{N}$ & $\mathbf{n}$ & \% \\
\hline Berlin (West) & 118 & 178 & $" 150,8 "$ \\
\hline Hamburg & 109 & 110 & $700,9 "$ \\
\hline Niedersachsen & 487 & 379 & 76,3 \\
\hline Bremen & 59 & 45 & 66,9 \\
\hline Schleswig-Holstein & 175 & 117 & 59,7 \\
\hline Baden-Würtemberg & 516 & 308 & 52,3 \\
\hline Bayern & 767 & 401 & 48,9 \\
\hline Nordrh.-Westfalen & 1987 & 972 & 44,4 \\
\hline Hessen & 313 & 139 & 29,4 \\
\hline Saarland & 85 & 25 & 20,1 \\
\hline Rheinland-Pfalz & 363 & 73 & \\
\hline
\end{tabular}

[modifiziert nach Kleemann et al.1997c].

\subsubsection{SIDS-typische Obduktionsbefunde}

Auch wenn sich bei der Obduktion von Plötzlichen Kindstodfällen definitionsgemäß keine Todesursache nachweisen läßt, sondern der Plötzliche Säuglingstod eine Ausschlußdiagnose ist, so gibt es doch einige typische Organbefunde, die gehäuft bei SIDS-Fällen zu finden sind, den Tod des Kindes jedoch nicht erklären können.

Diese Befunde findet man auffallend regelmäßig, obwohl sie ansonsten in dieser Form eher ungewöhnlich sind. Eine entsprechende Kombination solcher Befunde ist daher geeignet, die Diagnose "Plötzlicher Säuglingstod" wahrscheinlicher zu machen [Wilske 1984].

- Äußeres Erscheinungsbild:

Die verstorbenen Kinder sind augenscheinlich meist normal entwickelt, gut ernährt und gepflegt [Kleemann et al. 2000b; Valdes-Dapena 1992]. Oft weisen die Säuglinge eine Zyanose der Lippen und Nagelbetten auf [Valdes-Dapena 1992]. Die Kinder sind bei Auffindung oft stark verschwitz, teilweise ist sogar das Bettzeug oder die Matratze durchnäßt. Wilske berichtet von Untersuchungen, nach denen bei über $80 \%$ der 
Plötzlichen Säuglingstodesfälle eine finale Hypertermie nachgewiesen werden konnte, die teilweise bis zu $42^{\circ} \mathrm{C}$ erreichte [Wilske 1984]. Kleemann et al. fanden bei etwa $38 \%$ der verstorbenen Kinder Hinweise auf starke prämortale Schweißausbrüche [Kleemann et al. 1991].

Es fällt auf, daß sich bei zirka $40-60 \%$ der verstorbenen Kinder ein weißliches, manchmal auch blutig durchsetztes, zum Teil bereits angetrocknetes, schaumiges Sekret in den Nasenöffnungen befindet, welches als Hinweis auf ein hämorrhagisches Lungenödem zu deuten ist. Dieses Sekret ist auch im Pharynx und Larynx, in der Trachea und im Bronchialbaumsystem $\mathrm{zu}$ finden [Kleemann et al. 2000b]. Ein hämorrhagisches Lungenödem findet sich sonst infolge zunehmenden Atemversagens gehäuft nach protrahierter agonaler Phase, beispielsweise bei Opiatintoxikationen, und bei Grippepneumonien [Penning et al. 1996]. Valdes-Dapena führt die Flüssigkeit in den Nasenöffnungen auf eine Kontraktion der relativ elastischen, kindlichen Lungen zurück: die Alveolarepithelien der kindlichen Lungen seien dicker als die von Erwachsenen, deswegen hätten die kindlichen Alveolen die Tendenz, nach dem Tod zu kollabieren. Alveolen von SIDS-Kindern enthielten oft ödematöse Flüssigkeit, teilweise auch geringe Mengen Blut. Demzufolge werde die Intraalveolarflüssigkeit aus den Atemwegen über die Trachea, Larynx und schließlich die Nase quasi ausgepresst [Valdes-Dapena 1992].

- Gehirn:

Man findet bei der Obduktion meist ein ausgeprägtes Hirnödem [Penning et al. 1996]. Die Ventrikel sind verschmälert und die Gefäße der Meningen oft stark gestaut [Wilske 1984].

- Thymus:

Wilske beschreibt einen meist sehr großen Thymus, der bis über den Herzbeutel reicht. Kleinere Thymi seien meist mit Infekten oder anderen schweren Beeinträchtigungen kombiniert [Wilske 1984]. Nach Valdes-Dapena hingegen ist der Thymus bei SIDSFällen nicht vergrößert, aber oft übersäht von Petechien. Diese Thymus-Petechien gehören wohl zu den bekanntesten SIDS-Befunden, sind jedoch nicht immer nachweisbar und nicht SIDS-spezifisch, sondern werden auch bei anderen Todesarten, 
wie z.B. Asphyxie, gefunden, allerdings selten in sehr goßer Menge. Nach makroskopischen und mikroskopischen Untersuchungen finden sich in 69\% der SIDSFälle und in 38\% der Kontrollfälle Thymuspetechien. Bei rund 20\% der SIDS-Fälle kann man weder makroskopisch, noch mikroskopisch Punktblutungen im Thymus nachweisen [Valdes-Dapena 1992]. Risse et al. verglichen histologisch die ThymusPetechien von SIDS-Fällen und Nicht-SIDS-Fällen: Bei erstickten Kindern (äußeres Ersticken) waren die Petechien in der Regel nur spärlicher vorhanden als bei SIDSKindern. Auch bei anderen Nicht-SIDS-Fällen unterschieden sich die Punktblutungen von denen beim SIDS: sie waren unterschiedlich groß und unregelmäßig über Mark und Rinde verteilt, wähend sie bei SIDS-Kindern bevorzugt im kortikalen Bereich zu finden sind und eher von regelmäßiger Größe sind [Risse et al. 1989].

- Respirationstrakt:

Oft finden sich Schleimhautrötungen der oberen Atemwege, die zum Teil mit einer Lymphknotenvergrößerung einhergehen [Kleemann et al. 2000b]. Auch Valdes-Dapena fand bei eigenen Untersuchungen heraus, daß die verstorbenen Kinder gehäuft eine subakute Infektion des oberen Respirationstraktes aufwiesen. Bei 40\% der SIDS-Fälle ließ sich mikroskopisch eine Epiglottitis nachweisen, eine Tracheitis fand sich bei $29 \%$ der Kinder. Auch eine Laryngitis war bei den SIDS-Fällen ein relativ üblicher Befund. Anamnestisch gaben die Eltern bei 44\% der verstorbenen Kinder an, das Kind habe innerhalb der letzten zwei Wochen vor dem Tod an einem Atemwegsinfekt gelitten [Valdes-Dapena 1992].

Die Lungen sind blutreich und meistens gebläht, ödematös und fleckig gezeichnet, was sich auf dys- oder atelektatische Bezirke einerseits und emphysematische Bereiche andererseits zurückführen läßt. Auch eine Hämorrhagie oder ein hämorrhagisches Lungenödem kann eine fleckige Zeichnung verursachen [Kleemann et al. 2000b; Valdes-Dapena 1992; Wilske 1984]. Valdes-Dapena beschrieb makroskopisch und mikroskopisch sichtbare pleurale Petechien bei SIDS-Fällen in 63\%, bei den Kontrollen hingegen nur bei $38 \%$. Sie seien vermutlich agonal entstanden. Die teilweise massive, intrakapillare Blutstauung in den Lungen sei nicht spezifisch für SIDS, sondern würde auch bei $80 \%$ der Kontrollfälle auftreten [Valdes-Dapena 1992]. 
- Herz:

Die Herzmuskulatur ist von fahler Farbe [Penning et al. 1996], im Herzen befindet sich ausschließlich flüssiges Leichenblut [Valdes-Dapena 1992]. Oft findet sich eine akute Rechtsherzdilatation und gelegentlich vermehrt Perikardflüssigkeit. Subepikardiale Petechien -besonders an der Herzbasis unter Bevorzugung der rechten Seite- sind häufig zu sehen, können aber auch ganz fehlen [Wilske 1984].

- Leber, Pankreas, Magen-Darm-Trakt:

Die Leber ist meist von fleckiger, gelblich-bräunlicher Farbe. Hepatische ErythropoeseHerde sind bei SIDS-Fällen (23\%) signifikant häufiger $\mathrm{zu}$ finden als bei Kontrollen (14\%) [Valdes-Dapena 1992].

SIDS-typische makroskopische Auffälligkeiten am Magen, Pankreas und Darm werden in der Literatur nicht beschrieben.

- Milz:

Die Milz ist eher blutarm, eine Milzkörperchenzeichnung gut abgrenzbar [Kleemann et al. 2000b; Wilske 1984].

- Nieren, Harnblase:

Die Nieren sind äußerlich meistens blass, erreichen aber noch keine Organeigenfarbe. Zurückführen lassen sich die blassen Nieren, ebenso wie die blutarme Milz, fleckige Leber und das fahle Myokard, möglicherweise auf eine Zentralisation infolge länger andauernden Streßzustandes mit Katecholaminausschüttung, ähnlich einem Schockzustand. Auf der Schnittfläche der Nieren läßt sich oft eine blasse Rinde von einem blutreicherem, und damit dunklerem Mark abgrenzen [Wilske 1984]. Die Harnblase ist in der Regel leer [Kleemann et al. 2000b; Valdes-Dapena 1992; Wilske 1984]. 


\subsubsection{SIDS versus Tötungsdelikt}

"Differentialdiagnostisch besteht bei der Obduktion die Schwierigkeit bzw. nahezu Unmöglichkeit, gewaltsame Todesfälle durch weiche Bedeckung, also Aufdrücken eines Kissen, feuchten Tuches o.ä. sicher abzugrenzen. Hinweise könnten allenfalls Erstickungsblutungen im Gesichtsbereich bieten, die aber z.B. auch durch Reanimation erklärbar sind. Bei rein vorsichtshalber durchgeführten Obduktionen offensichtlicher SIDS-Kinder werden nicht ganz selten (bis $\mathrm{zu}$ ca.10\%) völlig unerwartet tödliche Mißhandlungen festgestellt!" [Penning et al. 1996].

Auch nach Valdes-Dapena ist eine Unterscheidung zwischen einer Tötung durch Ersticken, zum Beispiel mittels eines weichen Gegenstandes, und einem SIDS nicht möglich. Die makroskopischen und mikroskopischen Befunde sind dieselben [ValdesDapena 1992; Kleemann 1997a].

Das Ersticken ist wohl die häufigste übersehene Methode der Kindstötung. Bei Säuglingen und Kleinkindern ist das Ersticken eine relativ leicht auszuführende Tötungsmethode. Zum einen werden Kinder durch die Hände des Täters, zum anderen auch durch Niederdrücken des Kindes in das Bettzeug oder ähnliches erstickt. Somit ist derzeit eine Unterscheidung zwischen natürlichen und kriminellen Plötzlichen Todesfällen im Säuglings- und Kleinkindesalter nicht immer möglich [Roll 2000].

Ein Sonderfall unter den Tötungen von Säuglingen und Kleinkindern ist das Münchhausen-Syndrom-by-proxy. Hierbei wird durch elterliche Manipulation, meistens durch die Mutter, das Kind absichtlich "krank gemacht", sei es durch intoxikieren (Medikamenten- oder Giftbeibringung) oder durch sogenanntes “Anersticken”. Die psychisch kranke Mutter zieht einen Gewinn aus der Situation, indem sie bei den häufigen Arztbesuchen und Hospitalisierungen des Kindes als besonders fürsorglich und umsorgend auftritt, und so Zuwendung und Mitleid wegen der Belastungen durch ihr augenscheinlich schwerkrankes Kind von Seiten des Pflegepersonals und der Ärzte zu erhalten versucht. Das Münchhausen-Syndrom-by-proxy endet für das betroffene Kind nicht selten mit dem Tod, falls nicht rechtzeitig durch Dritte die Situation erkannt wird [Bouden et. al 1996]. Ein auffallendes Merkmal ist die häufige Inanspruchnahme ärztlicher Hilfe unter Vorbringung zum Teil kaum nachvollziebarer Anamnesen [Penning et al. 1996]. 
Valdes-Dapena berichtet über eine Untersuchung durch Meadow im Jahr 1990 von 27 durch ihre Mütter erstickten Kindern. In elf Fällen litt die Mutter an einem Münchhausen-Syndrom-by-proxy. Bei diesen Kindern begannen die "Anerstickungen" durch die Mutter in einem Alter von einem bis drei Monaten und dauerten an, bis sie von jemand anders entdeckt wurden oder das Kind verstarb, in der Regel nach sechs bis zwölf Monaten. Möglicherweise hat das Kind, bis die Situation aufgedeckt wird, bereits eine Hirnschädigung. Oft sind in der Familie bereits ältere Geschwister plötzlich und unerwartet verstorben, diese Todesfälle wurden jedoch als SIDS eingestuft [ValdesDapena 1992].

In vielen Veröffentlichungen wird über ein bis zu sechsfach erhöhtes SIDS-Risiko von Geschwistern verstorbener Kinder berichtet. Diese Angaben basieren unter anderem auf der Beobachtung, daß in SIDS-Familien manchmal mehr als ein SIDS-Fall auftritt. Bei solchen Untersuchungen und den daraus gezogenen Schlüssen sollte immer auch bedacht werden, ob nicht möglicherweise in Einzelfällen ein Münchhausen-Syndromby-proxy die Ursache für mehrfache Säuglingstodsfälle in einer Familie sein könnte.

Eine weitere Form des Kindesmißhandlung, die äußerlich nicht sichtbar sein muß, aber durch eine Obduktion sicher aufgedeckt werden kann, ist das Schütteltrauma. Hier kommt es durch massives Schütteln oder Schlagen des Kopfes gegen eine Unterlage [Roll 2000] zu subduralen Blutungen durch Einrisse der Brückenvenen zwischen Arachnoidea und venösen Sinus [Penning et al. 1996], mit oft tödlichem Ausgang.

Ferner gibt es noch das sogenannte "Battered Child Syndrome", eine Form der chronischen Kindesmißhandlung, die sich über lange Zeiträume erstrecken kann. Man kann frische und ältere Verletzungen nebeneinander beobachten, wobei diese sich von Knochenbrüchen über Verbrennungen, Unterernährung und Exsikkosen bis hin zu schwersten Traumata der Bauchorgane durch stumpfe Gewalt erstrecken [Roll 2000]. Im Jahr 1983 lag laut Roll die Tötungsrate bei Weißen der amerikanischen Bevölkerung bei 3,6 pro 100.000 Kinder, die jünger als ein Jahr waren. In Österreich wurden im Jahr 1993 pro 100.000 Kinder unter einem Jahr 3,2 Tötungsdelikte aufgedeckt [Roll 2000]. 


\subsection{Die BMBF-Studie "Plötzlicher Säuglingstod"}

Von Januar 1999 bis Ende Oktober 2001 nahm das Institut für Rechtsmedzin der Universität München als eines von zu Studienende 14 Studienzentren verschiedener Regionen Deutschlands an der multizentrischen, bundesweiten Studie "Plötzlicher Säuglingstod" teil. Diese auf drei Jahre angesetzte Studie wurde mit Mitteln des Bundesministeriums für Bildung, Wissenschaft, Forschung und Technologie (BMBF) gefördert und unter Leitung der Westfälischen Wilhelms-Universität in Münster durchgeführt.

Die im Folgenden dargestellte Beschreibung der Studie basiert auf dem Studienhandbuch der Hauptphase 1998 - 2003 [Westfälische Wilhelms-Universität Münster 2000].

\subsubsection{Studienteile und -ziele}

Die Studie gliederte sich in einen Hauptteil in Form einer Fall-Kontroll-Studie und mehrere Teilprojekte. Sie wurde in sechs Regionen Deutschlands an -zunächst- 13 Studienzentren unter Mitarbeit von Rechtsmedizinern, Pädiatern, Epidemiologen, Interviewern und Selbsthilfegruppen durchgeführt.

Die Teilprojekte waren im Einzelnen:

- Death-Scene-Investigation (unter Leitung der medizinischen Hochschule Hannover)

- Virusinfekt

- Toxikologie und Objektivierung des Risikofaktors Rauchen

- Neuropathologie

- Totenscheinanalyse

- Familienbegleitung

- Aufbau eines Referenzzentrums für zukünftige wissenschaftliche Auswertungen

Das übergeordnete Ziel der Studie "Plötzlicher Säuglingstod" war die Erarbeitung von Präventionsempfehlungen als Ansatz für eine weitere Reduktion der SIDS-Inzidenz. 
Um dieses Ziel mittels einer ausreichenden Datengrundlage verwirklichen zu können, wurden die folgenden Einzelziele definiert:

- Senkung der Säuglingssterblichkeit

- Exakte Bestimmung der SIDS-Inzidenz

- Berechnung von Interaktionen und Kofaktoren

- Elternbetreuung

- Bessere Identifikation von Hochrisikokindern

- Erarbeitung effektiver Präventionsprogramme

- Realisation einer überregional gültigen Studie

- Standortbestimmung der SIDS-Epidemiologie und -Pathogenese

\subsubsection{Studiendesign}

Bei dieser Studie handelte es sich um eine Fall-Kontroll-Studie. Sie bestand zum einen aus der Datenerhebung durch medizinische Unterlagen und ein Interview mit den Eltern des verstorbenen Kindes, zum anderen aus der Obduktion.

Dem Elterninterview lag ein standardisierter Fragebogen mit 96 Fragen zugrunde, das Interview wurde von eigens dafür geschulten Interviewern durchgeführt.

Die medizinischen Daten wurden aus dem Mutterpaß und eventuell vorhandenen Arztbzw. Krankenhausunterlagen erhoben.

Die Obduktion wurde gemäß eines Standardautopsieprotokolls durchgeführt, um eine einheitliche phänomenologische Beschreibung und Befunderhebung zu gewährleisten. Um die Ziele der Studie erfüllen zu können sollte eine genügend große Fallzahl untersucht werden. Es wurde angestrebt, 600 SIDS-Fälle und die Kontrollfälle in die Studie aufzunehmen.

Jedem erfaßten SIDS-Fall wurden drei, über die zuständigen regionalen Einwohnermeldeämter rekrutierte, alters- und geschlechtsgematchte Kontrollkinder gegenübergestellt. 


\subsubsection{Ein- und Ausschlußkriterien}

Die in die Studie aufgenommenen Kinder mußten zwischen acht und 365 Tage alt sein. Die SIDS-Fälle durften nicht an vor dem Tod bekannten, sterberelevanten Krankheiten gelitten haben, keine definierte Todesursache aufweisen und mußten nach der Geburt zuhause gelebt haben, also das Krankenhaus nach der Geburt verlassen haben.

Die Kontrollfälle durften sich zusätzlich zum Interviewzeitpunkt nicht in Krankenhausbehandlung befinden, nicht lebensbedrohlich krank sein und keine Zwillingsgeschwister von verstorbenen SIDS-Fällen sein.

\subsection{Ziele dieser Arbeit}

Im Rahmen dieser Arbeit sollen folgende Fragestellungen untersucht werden:

- Stehen die in München erhobenen epidemiologischen Daten mit den in der internationalen Literatur beschriebenen Statistiken in Einklang?

- Gibt es histologisch nachweisbare pathologisch-anatomische Veränderungen, die autoptisch/ makroskopisch dem Nachweis entgangen wären? Lassen sich die im Rahmen der Obduktion aufgrund makroskopischer Veränderungen gestellten Verdachtsdiagnosen histologisch verifizieren bzw. verfeinern?

- Handelt es sich im Einzelfall abschließend um einen Plötzlichen Säuglingstod oder kann ein solcher sicher ausgeschlossen werden? Wie hoch ist demnach der Anteil der verifizierten SIDS-Fälle an der Gesamtzahl der mit Verdacht auf SIDS zur Obduktion gelangten Säuglinge? Gibt es überhaupt einen Plötzlichen Säuglingstod als eigenständiges Syndrom? 


\section{MATERIAL UND METHODEN}

\subsection{Epidemiologische Datenerhebung}

Anhand eines Arbeitsblattes, welches im Anhang abgebildet ist, wurden von dem bei der Obduktion anwesenden Kriminalbeamten zu jedem verstorbenen Säugling folgende Angaben erfragt und für die epidemiologischen Untersuchungen verwendet (Arbeitsblatt: siehe Anhang):

- Name

- Anschrift

- Geburtsdatum

- Wann wurde das Kind zuletzt lebend gesehen?

- Wann wurde das Kind tot aufgefunden?

- Lag das Kind bei Auffindung auf dem Bauch, Rücken oder der Seite?

- Wurde eine Reanimation durchgeführt? Von wem?

- War das Kind in letzter Zeit krank?

- Hatte das Kind Geschwister? Wieviele?

- Wenn ja, waren diese krank?

- Waren die Eltern krank?

- Wie alt sind die Eltern? Welchen Beruf üben sie aus?

- Rauchen die Eltern?

- Wie verlief die Schwangerschaft?

- In welcher Schwangerschaftswoche fand die Entbindung statt?

- Wurden bei dem Kind die erforderlichen Untersuchungen durchgeführt?

- Geburtsgewicht und Geburtsgröße des Kindes

- Zuletzt behandelnder Kinderarzt

- Welche Todesart hat der die Leichenschau durchführende Arzt in der Todesbescheinigung angekreuzt? 


\subsection{Histologische Untersuchungen}

Um eigenständig die umfassenden histologische Untersuchungen der einzelnen Fällen durchführen zu können, wurden bei der Obduktion bei allen Säuglingen Teile fast aller Organe entnommen, mikroskopische Präparate angefertigt und diese dann mikroskopisch untersucht.

Alle im Folgenden erwähnten technischen Geräte, Chemikalien und andere Materialien werden im Anhang detailliert aufgelistet.

\subsubsection{Entnommenes Gewebe und Fixation}

Die im Einzelnen bei der Obduktion entnommenen Teile waren: Teile aus weichem Gaumen mit Tonsilla palatina und Zungengrund, Trachea, Thyreoidea, Aorta, Ösophagus, Larynx, Halslymphknoten, allen Lungenlappen und zentralen Lungenanteilen, Herz, Thymus, Milz, Diaphragma, Glandula parotis, Glandula submandibularis, Bauchhaut, Magen mit Pylorus, Duodenum, Pankreas, Ileum und Coecum mit Appendix vermiformis, Colon ascendens, Leber, Niere, Nebennieren, Musculus iliopsoas, Nasenhöhle, Rippe, Hypophyse und Gehirn.

Die entnommenen Gewebe wurden zur Fixation sofort nach ihrer Entnahme für mindestens zwei bis maximal 14 Tage in 4\%iges gepuffertes Formaldehyd eingelegt. Eine Ausnahme bildeten die Teile des Gehirns, die für mindestens 14 Tage im Formaldehyd verblieben, um eine vollständige Durchfixation zu gewährleisten.

\subsubsection{Zuschnitt, Einbettung und Schnitt des Gewebes}

Die zugeschnittenen Gewebsstücke wurden in Einbettkapseln gelegt und in einen automatisch gesteuerten Einbettautomaten (Autotechnikon) gegeben. Dieser überführte innerhalb eines Zeitraums von 24 Stunden die Gewebeproben aus dem Medium Formaldehyd, über eine Alkoholreihe mit den Konzentrationen 70\%, 80\%, 90\% und 
100\% und Xylol, in das Medium Paraffin (flüssig). Das Gewebe wurde nun den Einbettkapseln wieder entnommen und in Paraffin eingebettet, das heißt es wurde in Ausgießförmchen gelegt und diese mit flüssigem, $70^{\circ} \mathrm{C}$ warmem Paraffin ausgegossen. Nach mehreren Stunden waren die Paraffinblöcke komplett ausgekühlt und konnten weiter verarbeitet werden. Sie wurden für einige Stunden in einem normalen Kühlschrank bei $+8^{\circ} \mathrm{C}$ gelagert, danach ebenfalls für mindestens einige Stunden bei $-24^{\circ} \mathrm{C}$ in einem Tiefkühlschrank tiefgefroren.

Von den tiefgefrorenen Paraffinblöcken wurden sodann mittels eines Schlittenmikrotoms Gewebsstreifen mit einer Schichtdicke von etwa drei $\mu$ heruntergeschnitten, welche auf einen Objektträger aufgelegt wurden. Die Objektträger wurden dann für etwa 24 Stunden in einem Wärmeschrank bei $68^{\circ} \mathrm{C}$ aufbewahrt, um das Gewebe fest auf den Objektträger zu binden. Danach wurden die Präparate auf den Objektträgern gefärbt.

Bei den Geweben, von denen eine Ölrotfärbung angefertigt werden sollte, unterschied sich die Vorgehensweise von der eben beschriebenen:

Diese Gewebeproben wurden nicht in Paraffin eingebettet, sondern gleich nach Entnahme aus dem Formaldehyd in einem Gefriermikrotom (Kryostat) bei $-30^{\circ} \mathrm{C}$ auf metallene Gefrierplättchen aufgefroren. Dann wurden mittels des Kryostaten fünf $\mu$ dicke Gewebsstreifen von den tiefgefrorenen Präparaten heruntergeschnitten, auf Objektträger gelegt und für etwa 24 Stunden an der Luft getrocknet, bevor sie einer Ölrotfärbung unterzogen wurden.

\subsubsection{Färbungen}

Die einzelnen Gewebe wurden zwecks Darstellung verschiedener Strukturen unterschiedlich gefärbt. Eine Übersicht gibt die folgende Tabelle (Tabelle 5). 
Tabelle 5: Obduktionsfälle BMBF-Studie, Institut $f$. Rechtsmedizin München 19992001: Übersicht über durchgeführte histologische Färbungen

\begin{tabular}{|l|l|}
\hline \multicolumn{1}{|c|}{ Färbung } & \multicolumn{1}{c|}{ Gefärbte Proben } \\
\hline Hämalaun-Eosin $(=\mathrm{HE})$ & alle Gewebe \\
\hline Alcianblau-PAS & Lunge \\
\hline Eisen (Berlinerblau-Reaktion = Fe) & Lunge, Milz \\
\hline Elastica-van-Giesson $(=\mathrm{EvG})$ & Larynx, Herz, Gehirn \\
\hline Giemsa & Rippe \\
\hline Ölrot & Herz, Leber, M. iliopsoas, Nebennieren \\
\hline Perjodsäure-Schiff-Reaktion $(=$ PAS $)$ & Niere \\
\hline
\end{tabular}

In der Regel, außer bei den Ölrot-Färbungen, wurden die Objektträger als erstes für 15 Minuten in Xylol entparaffiniert, dann in eine alkoholische Verdünnungsreihe mit Ethanol abnehmender Konzentration (erst 100\%, dann 97\% und 70\%) eingebracht, und schließlich dem eigentlichen Färbevorgang unterzogen. Die Rezepte für die jeweiligen Färbungen finden sich im Anhang.

Nach Abschluß des Färbevorganges wurden die Objektträger wieder in eine alkoholische Verdünnungsreihe, diesmal jedoch mit zunehmender Konzentration (erst 70\%, dann 97\% und 100\%) eingebracht und schließlich für zehn Minuten in Xylol entwässert.

Dann wurden die Präparate auf den Objektträgern mit einem Deckgläschen eingeschlossen, welches mittels eines speziellen Klebers aufgeklebt wurde.

Nach einer mindestens 24-stündigen Trocknungszeit konnten die Präparate mikroskopiert werden.

Die Ölrotfärbung unterschied sich dadurch von eben beschriebenem Vorgang, dass die Präparate nicht in Alkohol entwässert wurden, sondern in einem wäßrigen Medium verblieben und gefärbt wurden, um nicht das im Gewebe enthaltene Fett herauszulösen, sondern sichtbar zu machen. Der genaue Färbevorgang ist im Anhang dargestellt. 


\subsubsection{Mikroskopie}

Es folgt eine tabellarische Auflistung der von jedem Kind angefertigten Präparate mit Angabe der verwandten Färbungen. Die Präparate 27 bis 39 wurden ausschließlich für eine neuropathologische Begutachtung in der Studienzentrale angefertigt (Tabelle 6).

Tabelle 6: Obduktionsfälle BMBF-Studie, Institut f. Rechtsmedizin München 19992001: Übersicht über alle histologischen Präparate und ihre Färbungen

\begin{tabular}{|c|c|c|}
\hline Präparat Nr. & Material & Färbungen \\
\hline 1 & Tonsille, Gaumen, Uvula, Zunge & $\mathrm{HE}$ \\
\hline 2 & $\begin{array}{l}\text { Trachea, Glandula Thyreoidea, } \\
\text { Glandula Parathyreoidea, } \\
\text { Ösophagus }\end{array}$ & $\mathrm{HE}$ \\
\hline 3 & Bifurcatio & $\mathrm{HE}$ \\
\hline 4 & Larynx & $\mathrm{HE}, \mathrm{EvG}$ \\
\hline 5 & Lymphknoten (zervikal) & $\mathrm{HE}$ \\
\hline 6 & Lunge (alle fünf Lappen) & HE, Fe, Alzianblau-PAS \\
\hline 7 & Lunge, zentral & $\begin{array}{l}\text { HE, Fe, } \\
\text { Alzianblau-PAS }\end{array}$ \\
\hline 8 & Herz (Septumquerschnitt) & HE, EvG, Ölrot \\
\hline 9 & rechtes Herz (Längsschnitt) & $\mathrm{HE}, \mathrm{EvG}$ \\
\hline 10 & linkes Herz (Längsschnitt) & $\mathrm{HE}, \mathrm{EvG}$ \\
\hline 11 & Thymus & $\mathrm{HE}$ \\
\hline 12 & Milz & $\mathrm{HE}, \mathrm{Fe}$ \\
\hline 13 & Diaphragma & $\mathrm{HE}$ \\
\hline 14 & $\begin{array}{l}\text { Glandula Parotidea, } \\
\text { Glandula Submandibularis }\end{array}$ & $\mathrm{HE}$ \\
\hline 15 & Bauchhaut & $\mathrm{HE}$ \\
\hline 16 & Pylorus, Duodenum & $\mathrm{HE}$ \\
\hline 17 & $\begin{array}{l}\text { Duodenum, Pankreaskopf, } \\
\text { Pankreaskörper }\end{array}$ & $\mathrm{HE}$ \\
\hline 18 & Ileum, Caecum & $\mathrm{HE}$ \\
\hline 19 & Colon ascendens & $\mathrm{HE}$ \\
\hline 20 & Leber & HE, Ölrot \\
\hline 21 & Nieren, rechts und links & HE, PAS \\
\hline 22 & Nebennieren & HE, Ölrot \\
\hline 23 & Hypophyse & $\mathrm{HE}, \mathrm{EvG}$ \\
\hline 24 & Musculus iliopsoas & $\mathrm{HE}$ \\
\hline 25 & $\begin{array}{l}\text { Rippe mit } \\
\text { Knorpel-Knochen-Grenze }\end{array}$ & HE, Giemsa \\
\hline 26 & Conchae nasales & $\mathrm{HE}$ \\
\hline 27 & Gyrus frontalis & $\mathrm{HE}, \mathrm{EvG}$ \\
\hline 28 & Okzipitalpol & $\mathrm{HE}, \mathrm{EvG}$ \\
\hline
\end{tabular}




\begin{tabular}{|c|l|l|}
\hline Präparat Nr. & Material & Färbungen \\
\hline 29 & Großhirnmarklager periventrikulär & HE, EvG \\
\hline 30 & Hippocampus & HE, EvG \\
\hline 31 & Thalamus & HE, EvG \\
\hline 32 & Stammganglien & HE, EvG \\
\hline 33 & Epiphyse & HE, EvG \\
\hline 34 & Hypothalamus & HE, EvG \\
\hline 35 & Mesencephalon & HE, EvG \\
\hline 36 & Pons & HE, EvG \\
\hline 37 & Pyramide, Olive & HE, EvG \\
\hline 38 & Kleinhirn/ Nucleus dentatus & HE, EvG \\
\hline 39 & Rückenmark C1-C2 & HE, EvG \\
\hline
\end{tabular}

Alle Präparate, außer die des Gehirns, wurden auf jegliche pathologische Veränderung hin untersucht. Die erhobenen Befunde wurden auf einem Arbeitsblatt dokumentiert, um die histologischen Befunde der einzelnen Kinder vergleichen zu können.

\subsection{Bakteriologie}

Jedem verstorbenen Säugling wurden bei der Obduktion Abstriche, Gewebsproben und Körperflüssigkeiten für eine bakteriologische Untersuchung am Max-von-PettenkoferInstitut in München entnommen. Im einzelnen waren dies:

Abstriche von Nasopharynx, Trachea, Leptomeninx, Milz, Lunge peripher, eine abgebundene Dünndarmschlinge, Herzblut und eine Stuhlprobe.

\subsection{Virologie}

Eine virologische Untersuchung mittels PCR (Polymerase-Kettenreaktion) der Kinder wurde zentral in Erlangen durchgeführt, weshalb im Rahmen der Obduktion folgende Proben asserviert, tiefgefroren und in Trockeneis verpackt an die Studienzentrale weitergeleitet wurden: 
Herzblut, Liquor, Trachealabstrich, Nasopharynx, Leber, Lunge zentral und peripher, Rückenmark, Herz, Dünndarmschleimhaut, Dickdarmschleimhaut, Stuhlprobe und Herzbeutelflüssigkeit.

Routinemäßig erfolgte, sicherlich eine Schwäche des zentralen Studiendesigns, nur eine Untersuchung von Stuhlprobe und Trachealabstrich. Falls hier ein positiver Nachweis gelang, sollten auch die anderen Proben untersucht werden. Die Ergebnisse wurden uns nach Abschluß der Untersuchung zugesandt.

\subsection{Toxikologie, Ethanolbestimmung und Begleitstoffanalyse}

Von jedem Säugling wurden am hiesigen Institut im toxikologischen Labor anhand verschiedener Proben toxikologische Untersuchungen sowie eine Alkoholbestimmung und Begleitstoffanalyse mittels Head Space Gaschromatographie durchgeführt. Die asservierten Proben waren im Einzelnen:

Herzblut, Liquor, Blut aus der Oberschenkelvene, Urin, Augenkammerwasser, Gallenblase, Teile der Leber, Teile der Niere, Mageninhalt, Teile der Lunge und Unterhautfettgewebe.

\subsection{Weitere Untersuchungen}

Für eine geplante elektronenmikroskopische Untersuchung wurden folgende, ca. $3 \times 3 \times 3 \mathrm{~mm}$ große Proben asserviert, für 24 Stunden in Glutaraldehyd 2,5\% aufbewahrt, in einen Phosphatpuffer umgelagert und an die Studienzentrale verschickt: Teile von Herz, Niere, Leber, Thymus, Milz, zervikaler Lymphknoten und fünf Teile der Lunge.

Für eine Cotininbestimmung wurden dem verstorbenen Kind Haar, Herzbeutelflüssigkeit und Liquor entnommen und an die Studienzentrale weitergeleitet. 
Für die geplante Anlage einer Gewebebank wurde von jedem Kind periumbilikal eine Hautstanze entnommen, in ein Nährmedium gegeben und an das Institut für Humangenetik der Universität Münster weitergeleitet.

Teile der Leber und Unterhautfettgewebe wurden von der Studienzentrale für die geplante Anlage einer sogenannten „Umweltprobenbank“ angefordert.

\subsection{Abschließende Kategorisierung der Fälle}

Nach Abschluß sämtlicher Untersuchungen wurde für alle Kinder endgültig eine (Verdachts-)Diagnose für den Todeseintritt gestellt. Dazu wurden die durch die Anamneseerhebung gewonnenen Daten, die Befunde der Obduktion, der histologischen Untersuchung, der mikrobiologischen, virologischen und toxikologischen Untersuchungen abschließend begutachtet und die Kinder dann anhand eines von der Studienzentrale vorgegebenen Kategorisierungssystems beurteilt. Dazu wurden uns von der Studienzentrale vier Kategorien vorgegeben (Tabelle 7).

Tabelle 7: Obduktionsfälle BMBF-Studie, Institut f. Rechtsmedizin 1999-2001: Verwendete Kategorien für die abschließende Klassifikation der Todesfälle

\begin{tabular}{|c|ll|}
\hline Kategorie & $\begin{array}{l}\text { keine Auffälligkeiten in der Vorgeschichte } \\
1\end{array}$ & $\begin{array}{l}\text { - keine manifesten Krankheitszeichen in den letzten 48 Stunden } \\
\text { - keine frischen oder älteren pathologischen Autopsiebefunde }\end{array}$ \\
\hline $\begin{array}{c}\text { Kategorie } \\
2\end{array}$ & $\begin{array}{l}\text { leichte Auffälligkeiten/ Erkrankungszeichen während der letzten } \\
\text { achtundvierzig Stunden und/ oder: } \\
\text { - autoptisch nur pathologische Befunde bzw. Erkrankungen, die } \\
\text { üblicherweise nicht zum Tode führen }\end{array}$ \\
\hline $\begin{array}{c}\text { Kategorie } \\
3\end{array}$ & $\begin{array}{l}\text { pathologisch-anatomisch definierte Erkrankung mit möglicher } \\
\text { todesursächlicher Bedeutung }\end{array}$ \\
\hline $\begin{array}{l}\text { Kategorie } \\
4\end{array}$ & $\bullet \quad$ pathologisch-anatomisch gesicherte Todesursache \\
\hline
\end{tabular}




\subsection{Auswertung der gewonnenen Daten}

In Kapitel 3 erfolgt eine Auswertung der gesamten Daten aller obduzierten Kinder. Nach Durchführung der Kategorisierung in Kapitel 3.7. erfolgt eine Aufteilung der Fälle in SIDS-Fälle und Non-SIDS-Fälle.

In Kapitel 4 erfolgt dann eine Gegenüberstellung dieser in SIDS-Fälle und in NonSIDS-Fälle aufgetrennten Daten (Abb. 4).

Abb. 4: Obduktionsfälle BMBF-Studie, Institut f. Rechtsmedizin München 1999-2001: Bearbeitung der gewonnenen Daten

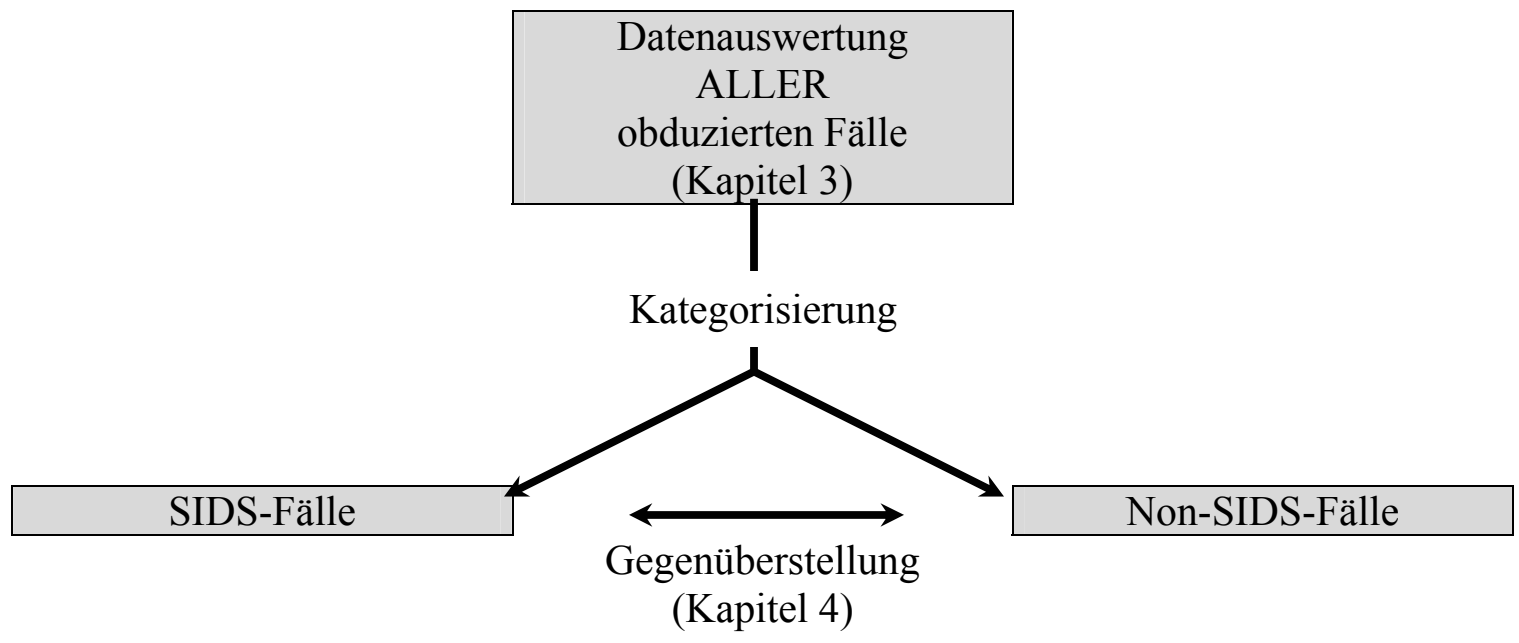




\section{ERGEBNISSE}

Am Institut für Rechtsmedizin der Universität München wurden im Studienzeitraum zwischen dem 01. Januar 1999 und dem 31. Oktober 2001 insgesamt 93 Säuglinge obduziert. Darunter befanden sich zwei von vornherein bekannte nicht-natürliche Todesfälle (Kohlenmonoxid-Intoxikation, Ersticken an einer Erdnuß), die in die folgenden Untersuchungen nicht aufgenommen wurden. Die anderen 91 Kinder wurden primär mit Verdacht auf SIDS von der Kriminalpolizei der Obduktion zugeführt. Die Kinder der Eltern, die der Studienteilnahme nicht zugestimmt hatten $(n=11)$, konnten nicht histologisch untersucht und somit auch nicht abschließend kategorisiert werden. Die epidemiologischen Daten dieser Kinder wurden jedoch in die entsprechenden Untersuchungen einbezogen, da es sich in allen Fällen um staatsanwaltschaftlich angeordnete Obduktionen handelte.

Da wir leider nicht in jedem Fall die kompletten Daten ermitteln konnten, wurde bei jeder der folgenden Untersuchungen die Anzahl (n) der einbezogenen Fälle innerhalb der Tabellen angegeben. Es handelt sich um eine zu geringe Fallzahl, um sinnvolle statistische Aussagen treffen zu können. Die Ergebnisse wurden daher entweder in absoluten Zahlen, in Prozent oder gegebenenfalls in beiden Formen angegeben. Die prozentualen Angaben wurden auf ganze Zahlen gerundet. Eine Tabelle mit sämtlichen gewonnen Falldaten findet sich im Anhang.

\subsection{Epidemiologische Untersuchungen}

In die folgenden Untersuchungen wurden alle mit Verdacht auf SIDS obduzierten Kinder einbezogen, auch die Kinder, die abschließend als Nicht-SIDS Fälle eingestuft werden mußten. 


\subsubsection{Studienteilnahme}

In die Studienteilnahme haben fast $88 \%$ der Eltern (80 von 91) eingewilligt, elf lehnten eine Studienteilnahme ab (Tabelle 8).

Tabelle 8: Obduktionsfälle BMBF-Studie, Institut f. Rechtsmedizin München 19992001: Studienteilnahme

\begin{tabular}{|l|c|}
\hline obduzierte Säuglinge & $91(100 \%)$ \\
\hline in Studienteilnahme eingewilligt & $80(88 \%)$ \\
\hline Studienteilnahme abgelehnt & $11(12 \%)$ \\
\hline
\end{tabular}

\subsubsection{Geschlecht}

Unter den 91 untersuchten Säuglingen fanden sich 39 Mädchen und 52 Jungen (Tabelle 9).

Tabelle 9: Obduktionsfälle BMBF-Studie, Institut f. Rechtsmedizin München 19992001: Geschlechtsverteilung

\begin{tabular}{|c|c|c|}
\cline { 2 - 3 } \multicolumn{1}{c|}{} & weiblich & männlich \\
\hline Anzahl & 39 & 52 \\
$(n=91)$ & $(43 \%)$ & $(57 \%)$ \\
\hline
\end{tabular}

\subsubsection{Alter}

Das folgende Diagramm gibt einen Überblick über das Alter der verstorbenen Kinder. Insgesamt waren Kinder im dritten und vierten Lebensmonat am häufigsten betroffen. Bei getrennter Betrachtung der Geschlechter fanden sich für die Mädchen Sterblichkeitsgipfel im zweiten/dritten und neunten/zehnten Lebensmonat. Bei den Jungen fanden sich die meisten Todesfälle im dritten/ vierten und siebten Lebensmonat (Abb. 5). 
Abb. 5: Obduktionsfälle BMBF-Studie, Institutf. Rechtsmedizin München 1999-2001: Altersverteilung

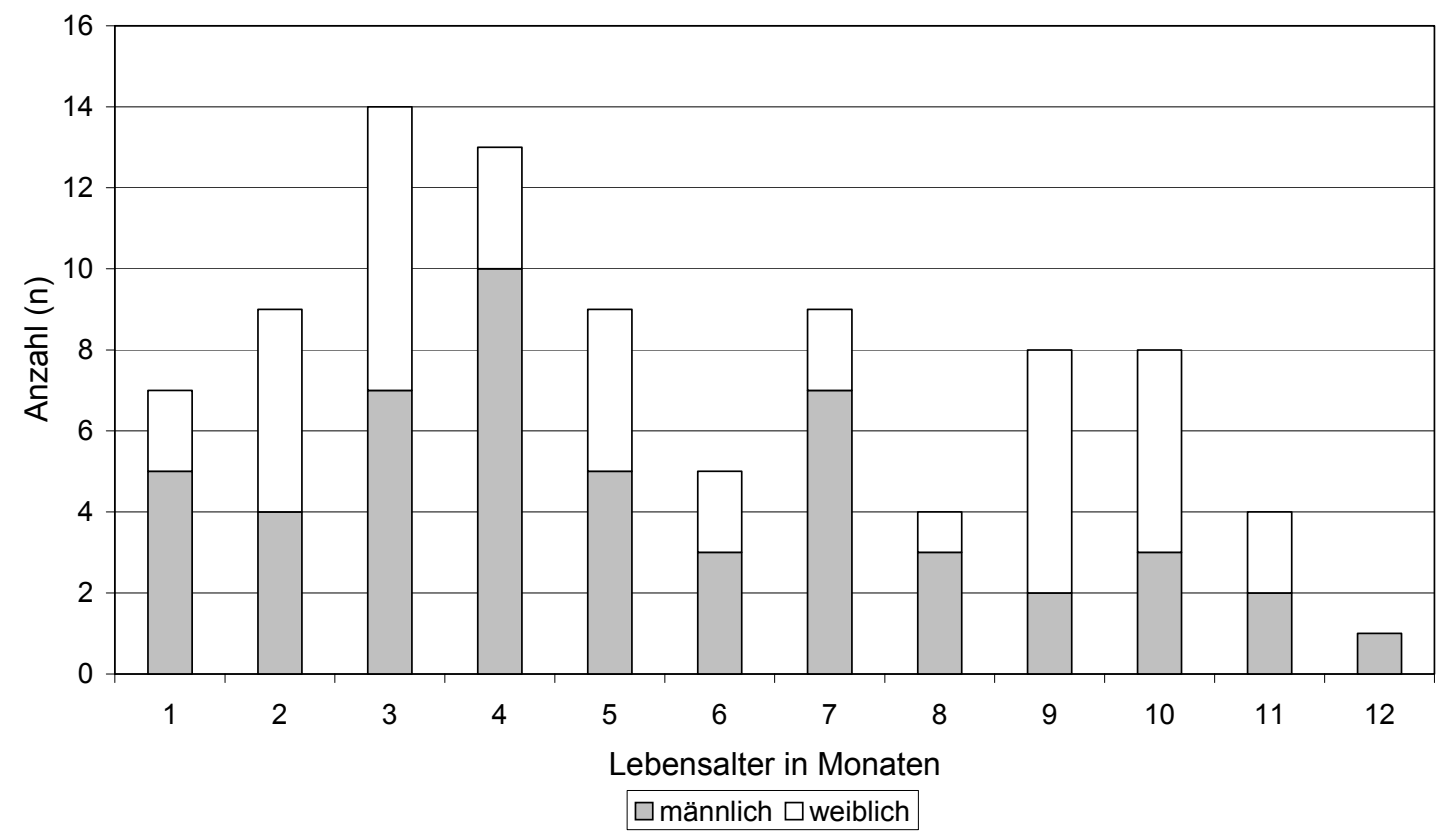

\subsubsection{Verteilung der Todesfälle auf die Wochentage}

Wir haben nur für wenige Kinder, die in direkter Anwesenheit der Eltern oder des Notarztes verstarben, eine genaue Sterbezeit. Bei den anderen Kindern liegen uns nur Daten dazu vor, wann sie tot aufgefunden wurden. Daher werden diese im Folgenden untersucht. Fast ein Drittel der Kinder verstarb an Wochenenden (33\%). Die meisten Todesfälle ereigneten sich an Samstagen (22\%) (Tabelle 10).

Tabelle 10: Obduktionsfälle BMBF-Studie, Institut f. Rechtsmedizin München 19992001: Wochentag des Todeseintritts

\begin{tabular}{|c|c|c|c|c|c|c|c|}
\cline { 2 - 7 } \multicolumn{1}{c|}{} & $\mathrm{Mo}$ & $\mathrm{Di}$ & $\mathrm{Mi}$ & $\mathrm{Do}$ & $\mathrm{Fr}$ & $\mathrm{Sa}$ & So \\
\hline $\begin{array}{c}\text { weiblich } \\
\left(\begin{array}{c}n=33= \\
100 \%)\end{array}\right.\end{array}$ & $\begin{array}{c}4 \\
(12 \%)\end{array}$ & $\begin{array}{c}7 \\
(21 \%)\end{array}$ & $\begin{array}{c}6 \\
(18 \%)\end{array}$ & $\begin{array}{c}2 \\
(7 \%)\end{array}$ & $\begin{array}{c}5 \\
(15 \%)\end{array}$ & $\begin{array}{c}4 \\
(12 \%)\end{array}$ & $\begin{array}{c}5 \\
(15 \%)\end{array}$ \\
\hline $\begin{array}{c}\text { männlich } \\
\left(\begin{array}{c}n=46= \\
100 \%)\end{array}\right.\end{array}$ & $\begin{array}{c}7 \\
(15 \%)\end{array}$ & $\begin{array}{c}5 \\
(11 \%)\end{array}$ & $\begin{array}{c}4 \\
(9 \%)\end{array}$ & $\begin{array}{c}7 \\
(15 \%)\end{array}$ & $\begin{array}{c}6 \\
(13 \%)\end{array}$ & $\begin{array}{c}13 \\
(28 \%)\end{array}$ & $\begin{array}{c}4 \\
(9 \%)\end{array}$ \\
\hline $\begin{array}{c}\text { gesamt } \\
(n=79= \\
100 \%)\end{array}$ & $\begin{array}{c}11 \\
(14 \%)\end{array}$ & $\begin{array}{c}12 \\
(15 \%)\end{array}$ & $\begin{array}{c}10 \\
(13 \%)\end{array}$ & $\begin{array}{c}9 \\
(11 \%)\end{array}$ & $\begin{array}{c}11 \\
(14 \%)\end{array}$ & $\begin{array}{c}17 \\
(22 \%)\end{array}$ & $\begin{array}{c}9 \\
(11 \%)\end{array}$ \\
\hline
\end{tabular}




\subsubsection{Jahreszeitliche Verteilung}

Da die Studie im Januar 1999 begann, aber im Oktober 2001, und nicht im Dezember, beendet wurde, konnten in diese Auswertung nur die Fälle aus den vollständigen Jahren 1999 und 2000, nicht aber aus dem unvollständigen Jahr 2001 aufgenommen werden, um eine Verzerrung der Ergebnisse zu vermeiden.

Insgesamt betrachtet zeigte sich eine Häufung der Todesfälle im Winter (Dezember bis Februar) und im Frühsommer (April bis Juli).

Betrachtet man jedoch die Geschlechter separat, so zeigt sich für Mädchen ein Sterblichkeitsgipfel im Februar gefolgt von den Monaten September und Dezember. Bei den Jungen hingegen verstarben besonders viele zwischen Mai und Juli, außerdem im Januar und Februar (Abb. 6).

Abb. 6: Obduktionsfälle BMBF-Studie, Institutf. Rechtsmedizin München 1999-2001: Jahreszeitliche Verteilung der Todesfälle

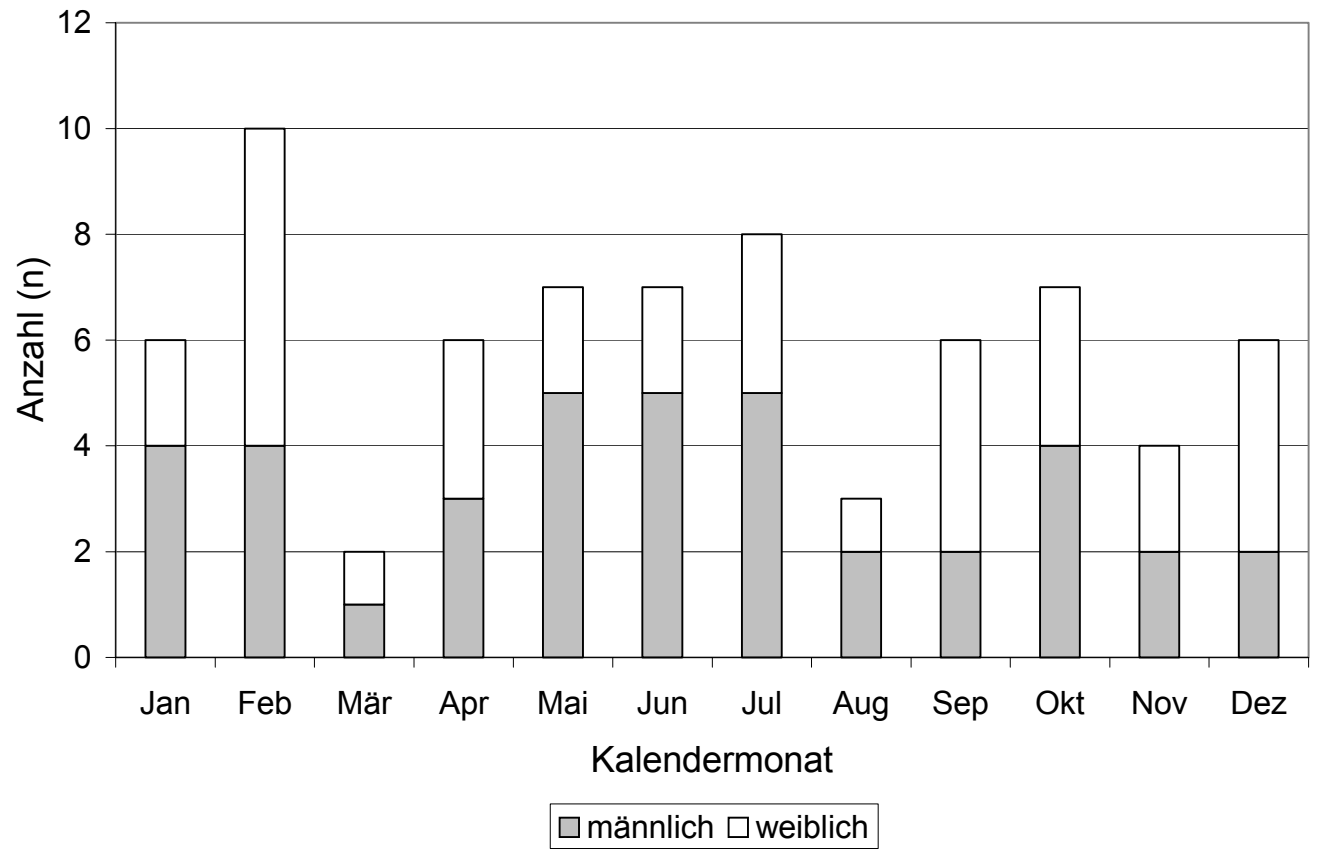




\subsubsection{Auffindesituation}

Der größte Teil der Säuglinge befand sich bei Auffindung in leblosem Zustand in Bauchlage (66\%). Am zweithäufigsten wurden die Kinder auf dem Rücken (27\%) und am dritthäufigsten in Seitenlage (7\%) aufgefunden.

Bei einem Teil der Kinder konnten wir die Auffindesituation nicht explorieren und in zwei Fällen waren die Kinder vermutlich, beziehungsweise angeblich auf dem Arm der Eltern verstorben, diese Fälle (insgesamt 15\%), wurden nicht in die folgende Tabelle aufgenommen (Tabelle 11).

Tabelle 11: Obduktionsfälle BMBF-Studie, Institut f. Rechtsmedizin München 1999_ 2001: Auffindesituation

\begin{tabular}{|c|c|c|c|}
\cline { 2 - 4 } \multicolumn{1}{c|}{} & Bauchlage & Rückenlage & Seitenlage \\
\hline weiblich & 23 & 10 & 2 \\
$(n=35=100 \%)$ & $(66 \%)$ & $(29 \%)$ & $(5 \%)$ \\
\hline männlich & 28 & 11 & 3 \\
$(n=42=100 \%)$ & $(67 \%)$ & $(26 \%)$ & $(7 \%)$ \\
\hline gesamt & 51 & 21 & 5 \\
$(n=77=100 \%)$ & $(66 \%)$ & $(27 \%)$ & $(7 \%)$ \\
\hline
\end{tabular}

\subsubsection{Schwangerschaftsdauer}

17\% der verstorbenen Kinder waren als Frühgeburten einzustufen, wurden also nach einer Schwangerschaftsdauer von weniger als 37 vollendete Schwangerschaftswochen geboren.

Bei getrennter Betrachtung der Geschlechter zeigte sich, dass von den verstorbenen Mädchen 10\% Frühgeburten waren, von den Jungen hingegen 23\%, also mehr als doppelt so viele (Tabelle 12). 
Tabelle 12: Obduktionsfälle BMBF-Studie, Institutf. Rechtsmedizin München 19992001: Schwangerschaftsdauer, geschlechtsbezogen

\begin{tabular}{|c|c|c|}
\hline & \multicolumn{2}{|c|}{ Schwangerschaftsdauer } \\
\hline & weniger als 37 Wochen & 37 und mehr Wochen \\
\hline $\begin{array}{c}\text { weiblich } \\
(n=21=100 \%)\end{array}$ & $\begin{array}{c}2 \\
(10 \%)\end{array}$ & $\begin{array}{c}19 \\
(90 \%)\end{array}$ \\
\hline $\begin{array}{c}\text { männlich } \\
(n=31=100 \%)\end{array}$ & $\begin{array}{c}7 \\
(23 \%)\end{array}$ & $\begin{array}{c}24 \\
(77 \%)\end{array}$ \\
\hline
\end{tabular}

\subsubsection{Größe und Gewicht zum Zeitpunkt der Geburt und der Obduktion}

Es wurden die Körpergewichte und -größen der verstorbenen Kinder zum Zeitpunkt der Geburt und der Obduktion ermittelt. Anhand von Somatogrammen, die in den von Pädiatern verwendeten Untersuchungsheften für Säuglinge enthalten sind, haben wir die Gewichts- und Größenangaben der Kinder in Bezug zu den üblicherweise verwendeten Perzentilen $(3 \%, 50 \%$ und $97 \%)$ gesetzt.

Zum Zeitpunkt der Geburt zeigt sich eine relativ gleichmäßige Verteilung, auch geschlechtsbezogen, im Bereich um die 50\%-Perzentile. Insgesamt waren sechs Kinder (drei Mädchen und drei Jungen) oberhalb der 3\%-Perzentile angeordnet und damit für die bestehende Körpergröße untergewichtig. Fünf Kinder (zwei Jungen und drei Mädchen) waren unterhalb der 97\%-Perzentile angesiedelt, und damit für ihre Größe übergewichtig (Abb. 7). Insgesamt waren 67\% Prozent der Kinder auf oder oberhalb der $50 \%$-Perzentile einzuordnen. 
Abb. 7: Obduktionsfälle BMBF-Studie, Institut f. Rechtsmedizin München 1999-2001: Perzentilen zum Zeitpunkt der Geburt

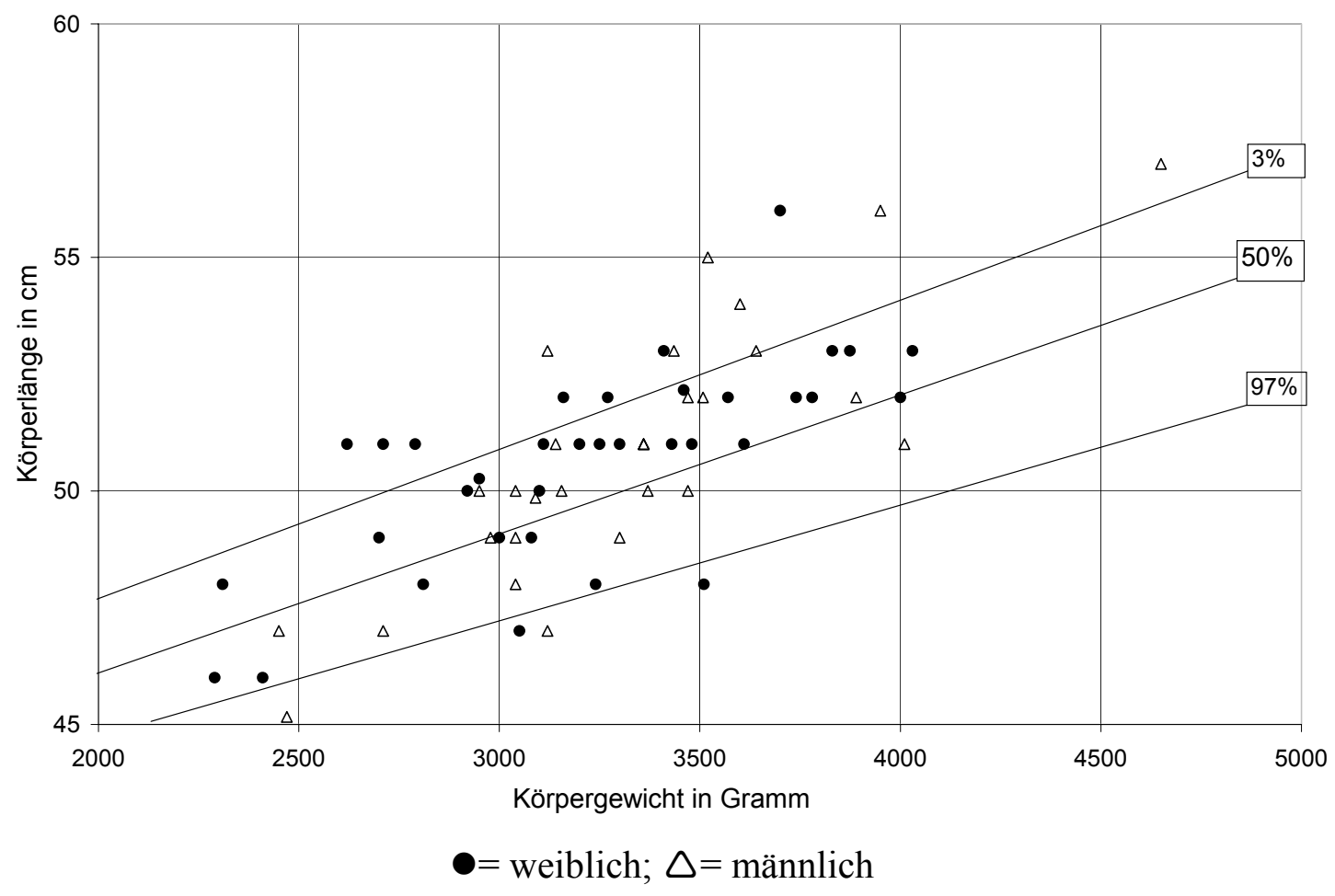

Auch unmittelbar vor der Obduktion wurden die Säuglinge gewogen und die Körperlänge gemessen. Trägt man diese Meßwerte in ein Somatogramm ein, so zeigt sich, dass über 90\% der Kinder (66 von insgesamt 73) zum Obduktionszeitpunkt auf oder oberhalb der 50\%-Perzentile anzuordnen waren, also relativ oder sogar absolut untergewichtig waren.

Bei genauerer Betrachtung zeigte sich, dass insgesamt 17 Säuglinge (acht Mädchen und neun Jungen) oberhalb der 3\%-Perzentile lagen, also absolut untergewichtig waren (insgesamt 23\%).

Nur ein einziges Kind (Mädchen) lag unterhalb der 97\%-Perzentile und war somit für seine Körpergröße übergewichtig. 97\% der Kinder waren auf oder oberhalb der 50\%Perzentile einzuordnen. Insgesamt schien kein relevanter Unterschied zwischen den beiden Geschlechtern zu bestehen (Abb. 8). 
Abb. 8: Obduktionsfälle BMBF-Studie, Institut f. Rechtsmedizin München 1999-2001: Perzentilen zum Zeitpunkt der Obduktion

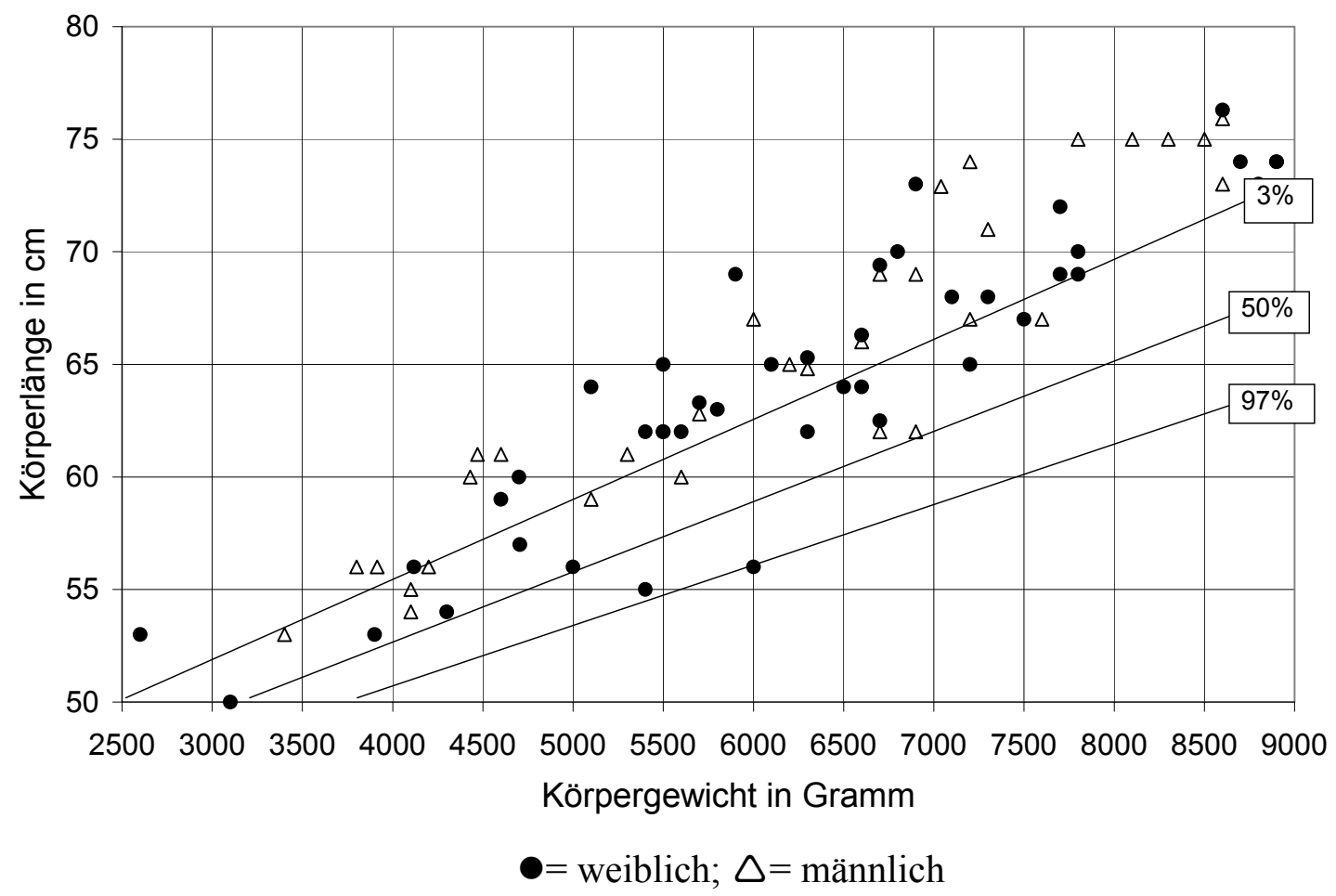

\subsubsection{Gesundheitszustand vor Todeseintritt}

Alle Eltern wurden durch die zuständigen Kriminalbeamten zum Gesundheitszustand des verstorbenen Kindes unmittelbar vor Todeseintritt befragt. Der größte Teil der Verstorbenen (53\%) war zum Zeitpunkt des Todeseintritts augenscheinlich gesund: den Eltern waren keine Anzeichen einer Erkrankung aufgefallen. An zweiter Stelle folgten mit $41 \%$ die Kinder, die zum Todeszeitpunkt an einer akuten Erkrankung (meist Schnupfen oder Erkältung) gelitten hatten. Am seltensten (7\%) waren Kinder, die zwar zum Todeszeitpunkt wieder gesund, jedoch noch kurz zuvor krank gewesen waren (Tabelle 13). 
Tabelle 13: Obduktionsfälle BMBF-Studie, Institut f. Rechtsmedizin München 19992001: Gesundheitszustand vor Todeseintritt

\begin{tabular}{|c|c|c|c|}
\cline { 2 - 4 } \multicolumn{1}{c|}{} & $\begin{array}{c}\text { krank unmittelbar } \\
\text { vor Todeseintritt }\end{array}$ & $\begin{array}{c}\text { krank in der letzten } \\
\text { Woche vor } \\
\text { Todeseintritt }\end{array}$ & $\begin{array}{c}\text { gesund seit } \\
\text { mindestens einer } \\
\text { Woche }\end{array}$ \\
\hline weiblich & 10 & 4 & 19 \\
\hline männlich & $(30 \%)$ & $(12 \%)$ & $(58 \%)$ \\
\hline$(n=41=100 \%)$ & 20 & 1 & 20 \\
gesamt & $(49 \%)$ & $(2 \%)$ & $(49 \%)$ \\
$(n=74=100 \%)$ & 30 & 5 & 39 \\
& $(40 \%)$ & $(7 \%)$ & $(53 \%)$ \\
\hline
\end{tabular}

Untersucht man den Gesundheitszustand für jedes Geschlecht separat, so scheinen Jungen (49\%) häufiger als Mädchen (30\%) aus akut krankem Zustand heraus verstorben zu sein (Tabelle 14).

Tabelle 14: Obduktionsfälle BMBF-Studie, Institut f. Rechtsmedizin München 19992001: Gesundheitszustand vor Todeseintritt, geschlechtsbezogen

\begin{tabular}{|c|c|c|c|}
\cline { 2 - 4 } & $\begin{array}{c}\text { krank unmittelbar } \\
\text { vor Todeseintritt }\end{array}$ & $\begin{array}{c}\text { krank in der letzten } \\
\text { Woche vor } \\
\text { Todeseintritt }\end{array}$ & $\begin{array}{c}\text { gesund seit mind. } \\
\text { einer Woche }\end{array}$ \\
\hline $\begin{array}{c}\text { weiblich } \\
(n=33=100 \%)\end{array}$ & 10 & 4 & 19 \\
\hline männlich & $(30 \%)$ & 1 & $(58 \%)$ \\
$(n=41=100 \%)$ & 20 & $(2 \%)$ & 20 \\
& $(49 \%)$ & & \\
\hline
\end{tabular}

\subsubsection{Geschwister}

Fünf der untersuchten Kinder waren Zwillinge (7\%), dabei handelte es sich um vier verstorbene Jungen und ein Mädchen. Bei 18 Kindern (26\%) handelte es sich um Einzelkinder, bzw. Erstgeborene. Alle anderen Kinder (74\%) hatten ältere Geschwister oder Zwillingsgeschwister (Tabelle 15). 
Tabelle 15: Obduktionsfälle BMBF-Studie, Institut f. Rechtsmedizin München 19992001: Anzahl der Geschwisterkinder

\begin{tabular}{|c|c|c|c|c|c|}
\cline { 2 - 6 } \multicolumn{1}{c|}{} & 0 & 1 & 2 & 3 & 4 und mehr \\
\hline $\begin{array}{c}\text { weiblich } \\
(n=32=100 \%)\end{array}$ & 9 & 17 & 4 & 2 & - \\
\hline männlich & $(28 \%)$ & $(53 \%)$ & $(13 \%)$ & $(6 \%)$ & \\
$(n=36=100 \%)$ & $(25 \%)$ & $(42 \%)$ & $(22 \%)$ & $(8 \%)$ & $(3 \%)$ \\
\hline gesamt & 18 & 32 & 12 & 5 & 1 \\
$(n=68=100 \%)$ & $(26 \%)$ & $(47 \%)$ & $(18 \%)$ & $(7 \%)$ & $(2 \%)$ \\
\hline
\end{tabular}

\subsubsection{Alter der Eltern bei der Geburt des verstorbenen Kindes}

In 88 Fällen konnten wir das Alter der Mutter, in 53 Fällen das des Vaters des verstorbenen Kindes bei dessen Geburt ermitteln. Junge Mütter waren wesentlich öfter vertreten als junge Väter. Insgesamt zeigt sich eine deutliche „Linksverschiebung“ des Alters der Mütter bezogen auf die Väter in den Altersgruppen bis zu 30 Jahren (Abb. 9).

Abb. 9: Obduktionsfälle BMBF-Studie, Institutf. Rechtsmedizin München 1999-2001: Alter der Eltern

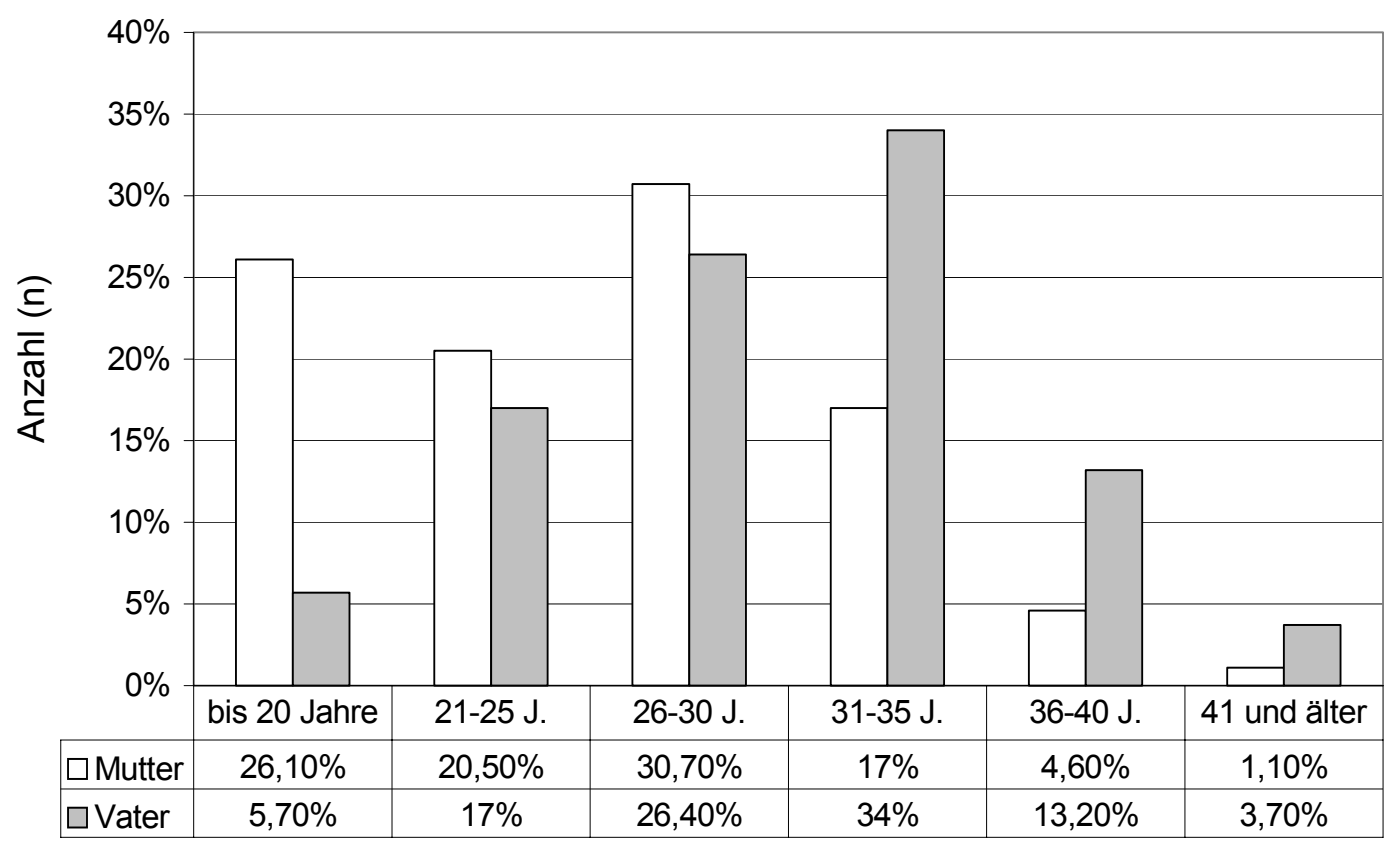

$\square$ Mutter $\square$ Vater 


\subsubsection{Soziale Stellung der Familie}

Der soziale Status einer Familie ist immer eng mit deren Einkommen, und dieses meist, natürlich nicht immer, mit dem Beruf der Eltern verknüpft.

Für die folgende Untersuchung wurden, falls vorhanden, beide Eltern als "Familie" zusammengefaßt und pro betroffener Familie jeweils der Elternteil mit dem höchsten Ausbildungsniveau betrachtet. Für dieses wurden vier verschiedene Unterteilungen angewandt (Gruppe 1-4):

Gruppe 1: Hausfrauen/-männer, Arbeitslose, Nichterwerbstätige

Gruppe 2: Hilfsarbeiter, Tätigkeiten ohne erfolgte Berufsausbildung

Gruppe 3: abgeschlossene Lehre

Gruppe 4: abgeschlossenes Studium an der Universität oder Fachhochschule

In Gruppe 1 bereiten die "Hausfrauen-/ männer" Schwierigkeiten, da wir nicht über Angaben verfügen, welche Berufsausbildung dieser Elternteil zuvor genossen hat. Es kann sich also beispielsweise um eine(n) arbeitslose(n) Sozialhilfeempfänger handeln, aber auch um eine Ärztin im Mutterschutz. Daher wurde die Gruppe 1 zwar in die folgenden Auswertungen der Vollständigkeit halber einbezogen, man kann ihr jedoch aufgrund der eingeschränkten Datenvorgaben nur beschränkte Bedeutung zukommen lassen.

Es zeigt sich, dass in den Familien der verstorbenen Kinder zu etwa einem Viertel (25\%) keiner der Eltern berufstätig war. In insgesamt 53\% (Spalte 1 und 2) der Fälle, also mehr als der Hälfte, handelte es sich um Familien, in denen einer oder beide Elternteile nicht berufstätig oder Hilfsarbeiter waren, also in der Regel eher als sozial schwach einzustufende Familien. Der größte Anteil der verstorbenen Kinder (39\%) stammte aus Familien, die der Gruppe drei zuzuordnen waren, also verfügte mindestens ein Elternteil über eine abgeschlossene Berufsausbildung und war auch berufstätig. In 8\% der Fälle war mindestens ein berufstätiger Akademiker vorhanden (Tabelle 16). 
Tabelle 16: Obduktionsfälle BMBF-Studie, Institut f. Rechtsmedizin München 19992001: Höchstes Ausbildungsniveau zumindest eines Elternteils

\begin{tabular}{|c|c|c|c|c|}
\cline { 2 - 5 } \multicolumn{1}{c|}{} & Gruppe 1 & Gruppe 2 & Gruppe 3 & Gruppe 4 \\
\hline weiblich & 8 & 9 & 8 & 3 \\
$(n=28=100 \%)$ & $(29 \%)$ & $(32 \%)$ & $(29 \%)$ & $(10 \%)$ \\
\hline männlich & 7 & 8 & 16 & 2 \\
$(n=33=100 \%)$ & $(21 \%)$ & $(24 \%)$ & $(48 \%)$ & $(7 \%)$ \\
\hline gesamt & 15 & 17 & 24 & 5 \\
$(n=61=100 \%)$ & $(25 \%)$ & $(28 \%)$ & $(39 \%)$ & $(8 \%)$ \\
\hline
\end{tabular}

Weiter wurde untersucht, ob sich ein Zusammenhang zwischen dem Ausbildungsniveau der Eltern und der Anzahl der vorhandenen Geschwister ergab.

In Familien mit einem oder zwei nicht berufstätigen Elternteilen (Gruppe 1) waren am häufigsten $(n=6)$ die Erstgeborenen verstorben (keine Geschwisterkinder). Die Todesfälle nahmen dann mit der Anzahl der Geschwister kontinuierlich ab.

Auch in Gruppe 2 verstarben bevorzugt Erstgeborene $(n=5)$ oder Kinder, die ein Geschwister hatten, also in der Regel wohl die Zweitgeborenen $(\mathrm{n}=7)$.

In Gruppe drei und vier waren in erster Linie die Kinder betroffen, die ein Geschwisterkind hatten ( $n=13$ beziehungsweise $n=4$ ), und zwar noch häufiger als die Kinder mit zwei oder drei Geschwistern (Tabelle 17).

Tabelle 17: Obduktionsfälle BMBF-Studie, Institut f. Rechtsmedizin München 19992001: Anzahl der Geschwisterkinder

\begin{tabular}{|c|c|c|c|c|c|}
\cline { 2 - 6 } \multicolumn{1}{c|}{} & \multicolumn{5}{c|}{ Anzahl der Geschwisterkinder } \\
\hline $\begin{array}{c}\text { höchstes } \\
\begin{array}{c}\text { Ausbildungs- } \\
\text { niveau eines } \\
\text { Elternteils }\end{array}\end{array}$ & 0 & 1 & 2 & 3 & 4 und mehr \\
\hline 1 & 6 & 4 & 3 & 1 & 1 \\
\hline 2 & 5 & 7 & 2 & 2 & - \\
\hline 3 & 4 & 13 & 4 & 1 & - \\
\hline 4 & 1 & 4 & 1 & - & - \\
\hline
\end{tabular}


Weiter wurde untersucht, ob die Kinder aus Familien, in denen beide Eltern berufstätig waren an anderen Wochentagen verstarben, als die Kinder aus Familien, in denen ein oder beide Elternteile nicht berufstätig waren.

In den Familien mit zwei Berufstätigen verstarben die Kinder gehäuft zu Wochenanfang (Montag und Dienstag). Bevorzugt an Donnerstagen stellten sich Todesfälle in den Familien ein, in denen nur ein Elternteil berufstätig war. Bei den Familien mit zwei Nichtberufstätigen zeigt sich keine Häufung an bestimmten Wochentagen (Tabelle 18).

Tabelle 18: Obduktionsfälle BMBF-Studie, Institut f. Rechtsmedizin München 19992001: Wochentag bei Totauffindung

\begin{tabular}{|c|c|c|c|c|c|c|c|}
\cline { 2 - 7 } \multicolumn{1}{c|}{} & Mo & Di & Mi & Do & Fr & Sa & So \\
\hline $\begin{array}{c}\text { beide } \\
\text { Eltern } \\
\text { arbeiten } \\
(n=18)\end{array}$ & $\begin{array}{c}6 \\
(33 \%)\end{array}$ & $\begin{array}{c}4 \\
(22 \%)\end{array}$ & $\begin{array}{c}2 \\
(11 \%)\end{array}$ & $\begin{array}{c}1 \\
(6 \%)\end{array}$ & $\begin{array}{c}1 \\
(6 \%)\end{array}$ & $\begin{array}{c}3 \\
(17 \%)\end{array}$ & $\begin{array}{c}1 \\
(5 \%)\end{array}$ \\
\hline $\begin{array}{c}\text { mind. ein } \\
\text { Elternteil } \\
\text { zu Hause } \\
(n=35)\end{array}$ & 4 & 5 & 4 & 6 & 8 & 5 & 3 \\
$(11 \%)$ & $(14 \%)$ & $(11 \%)$ & $(17 \%)$ & $(23 \%)$ & $(14 \%)$ & $(9 \%)$ \\
$\begin{array}{c}\text { beide } \\
\text { Eltern zu } \\
\text { Hause } \\
(n=7)\end{array}$ & $\begin{array}{c}1 \\
(14 \%)\end{array}$ & $\begin{array}{c}2 \\
(29 \%)\end{array}$ & $\begin{array}{c}2 \\
(29 \%)\end{array}$ & - & $\begin{array}{c}1 \\
(14 \%)\end{array}$ & $\begin{array}{c}1 \\
(14 \%)\end{array}$ & - \\
\hline
\end{tabular}

\subsubsection{3. „Postvitales“ Intervall}

Betrachtet man die Zeitspanne zwischen dem Moment, in dem das Kind letztmalig lebend gesehen wurde, und dem Zeitpunkt der Auffindung in leblosem Zustand (im folgenden als postvitales Intervall bezeichnet), so stellt man fest, dass der Tod des Kindes überwiegend innerhalb der ersten fünf Stunden nach dem „Zuletzt-lebendgesehen“ entdeckt wurde (58\%). 30\% der Eltern bemerkten das Ableben nach einem Zeitraum von sechs bis zehn Stunden und 6\% der Eltern erhielten über mindestens zehn Stunden kein Lebenszeichen ihres Kindes, bis sie dessen Ableben bemerkten (Tabelle 19). 
Tabelle 19: Obduktionsfälle BMBF-Studie, Institut f. Rechtsmedizin München 19992001: „Postvitales“ Intervall

\begin{tabular}{|c|c|c|c|c|}
\cline { 2 - 5 } \multicolumn{1}{c|}{} & weniger als $1 \mathrm{~h}$ & 1 bis $5 \mathrm{~h}$ & 6 bis $10 \mathrm{~h}$ & mehr als $10 \mathrm{~h}$ \\
\hline weiblich & 1 & 16 & 13 & 3 \\
$(n=33=100 \%)$ & $(3 \%)$ & $(49 \%)$ & $(39 \%)$ & $(9 \%)$ \\
\hline männlich & 3 & 23 & 7 & 1 \\
$(n=34=100 \%)$ & $(9 \%)$ & $(68 \%)$ & $(20 \%)$ & $(3 \%)$ \\
\hline gesamt & 4 & 39 & 20 & 4 \\
$(n=67=100 \%)$ & $(6 \%)$ & $(58 \%)$ & $(30 \%)$ & $(6 \%)$ \\
\hline
\end{tabular}

Betrachtet man jedoch die Geschlechter der Kinder getrennt voneinander, so zeigt sich, dass die Jungen früher, das heißt nach einem kürzeren Intervall, tot aufgefunden wurden als die Mädchen: Bei den Mädchen wurden 3\% innerhalb der ersten Stunde gefunden, bei den Jungen hingegen 8\%. Innerhalb von einer bis zu fünf Stunden wurden 49\% der Mädchen leblos entdeckt, aber 68\% der Jungen. Insgesamt blieb der Tod von Jungen kürzer unentdeckt als der von Mädchen, wie auch folgendes Diagramm zeigt (Abb. 10).

Abb. 10: Obduktionsfälle BMBF-Studie, Institut f. Rechtsmedizin München 1999-2001: „Postvitales" Intervall, geschlechtsbezogen

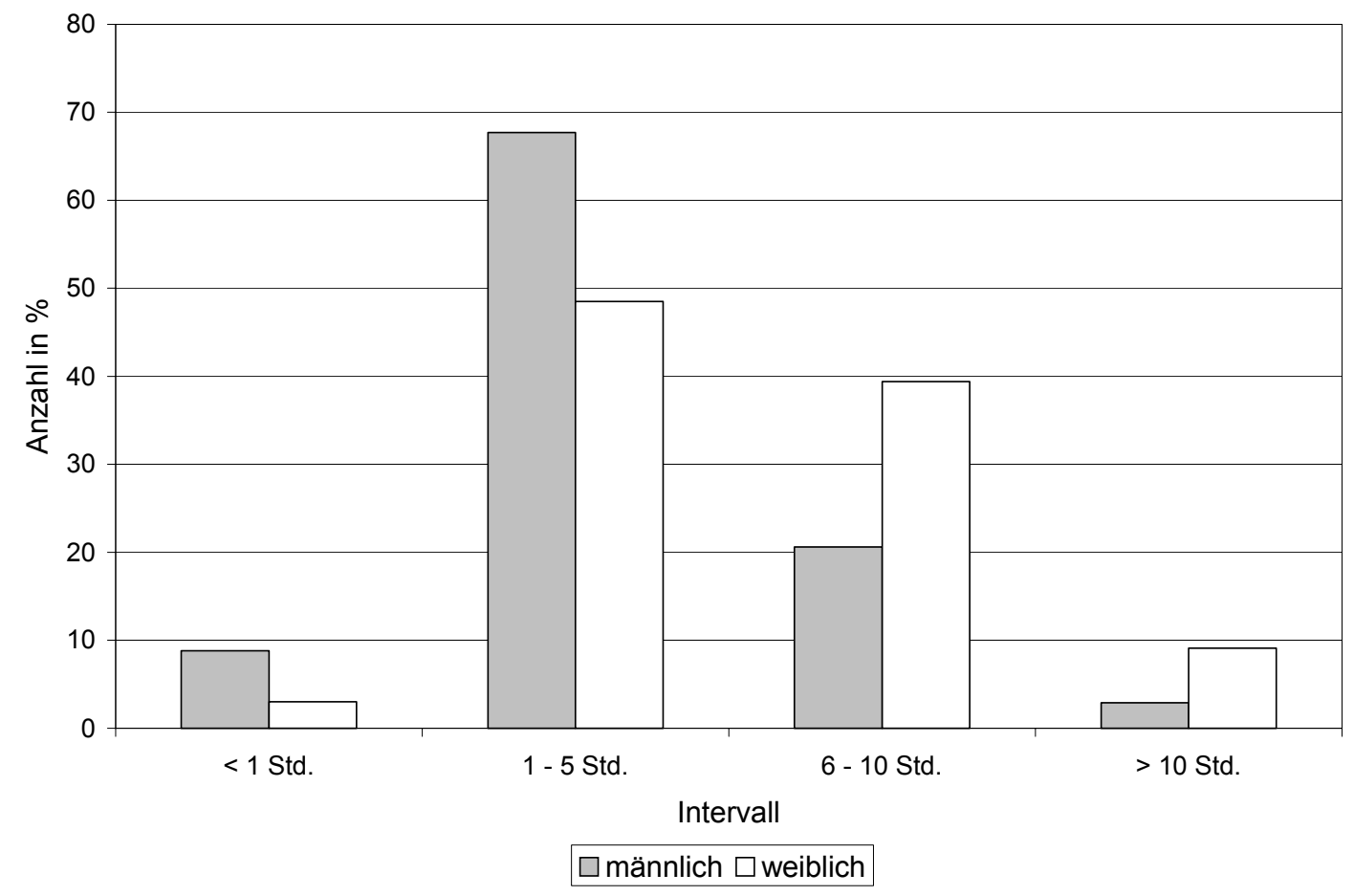


Dass sich das Alter der Mutter oder der soziale Status der Eltern, für den hier vereinfacht das Ausbildungsniveau herangezogen wurde, auf das „postvitale“ Intervall auswirken, zeigen die folgenden zwei Tabellen.

Die Mütter im Alter von bis zu 20 Jahren (die jüngste Mutter war 17 Jahre alt) fanden in $73 \%$ ihr verstorbenes Kind innerhalb der ersten fünf Stunden auf, also häufiger als die Mütter aller anderen Altersgruppen, wenn man von der einen Mutter zwischen 36 und 40 Jahren absieht. Mehr als zehn Stunden haben vor allem die Mütter der Altersgruppe zwischen 21 und 25 Jahren gebraucht, um den Tod des Kindes zu registrieren (Tabelle 20).

Tabelle 20: Obduktionsfälle BMBF-Studie, Institut f. Rechtsmedizin München 19992001: „Postvitales Intervall“ bezogen auf das Alter der Mutter bei der Geburt des Kindes

\begin{tabular}{|c|c|c|c|c|}
\cline { 2 - 5 } \multicolumn{1}{c|}{} & \multicolumn{4}{c|}{ Zeit bis zur Auffindung } \\
\hline Alter der Mutter & weniger als 1h & 1 bis $5 \mathrm{~h}$ & 6 bis $10 \mathrm{~h}$ & mehr als $10 \mathrm{~h}$ \\
\hline $\begin{array}{c}\text { bis 20 Jahre } \\
(n=15=100 \%)\end{array}$ & - & 11 & 3 & 1 \\
$21-25 \mathrm{Jahre}$ & $(73 \%)$ & $(20 \%)$ & $(7 \%)$ \\
$(n=14=100 \%)$ & $(7 \%)$ & $(50 \%)$ & $(29 \%)$ & 2 \\
\hline $26-30 \mathrm{Jahre}$ & 1 & 11 & 6 & 1 \\
$(n=19=100 \%)$ & $(5 \%)$ & $(58 \%)$ & $(32 \%)$ & $(5 \%)$ \\
\hline $31-35 \mathrm{Jahre}$ & 1 & 6 & 5 & - \\
$(n=12=100 \%)$ & $(8 \%)$ & $(50 \%)$ & $(42 \%)$ & - \\
\hline $\begin{array}{c}36 \text { bis } 40 \mathrm{Jahre} \\
(n=1=100 \%)\end{array}$ & - & 1 & - & - \\
\hline $\begin{array}{c}\text { allter als } 40 \mathrm{~J} . \\
(n=1=100 \%)\end{array}$ & - & $(100 \%)$ & & 1 \\
\hline
\end{tabular}

Betrachtet man das postvitale Intervall in Bezug auf das Ausbildungsniveau der Eltern, so erkannten die Eltern aus Gruppe 2 am häufigsten den Tod ihres Kindes innerhalb der ersten fünf Stunden (86\%), es folgten die Eltern der Gruppe 4 (67\%), Gruppe 3 (58\%) und schließlich die der Gruppe 1 (57\%) (Tabelle 21). 
Tabelle 21: Obduktionsfälle BMBF-Studie, Institut f. Rechtsmedizin München 19992001: „Postvitales “ Intervall bezogen auf das Ausbildungsniveau der Eltern

\begin{tabular}{|c|c|c|c|c|}
\cline { 2 - 5 } \multicolumn{1}{c|}{} & \multicolumn{4}{c|}{ Zeit bis zur Auffindung } \\
\hline $\begin{array}{c}\text { höchstes Aus- } \\
\text { bildungsniveau } \\
\text { der Eltern }\end{array}$ & weniger als 1h & 1 bis $5 \mathrm{~h}$ & 6 bis $10 \mathrm{~h}$ & mehr als $10 \mathrm{~h}$ \\
\hline $\begin{array}{c}\text { Gruppe } 1 \\
(n=14=100 \%)\end{array}$ & 1 & 7 & 6 & - \\
\hline Gruppe 2 & 2 & $(50 \%)$ & $(43 \%)$ & 1 \\
$(n=14=100 \%)$ & $(14 \%)$ & $(71 \%)$ & $(7 \%)$ & $(7 \%)$ \\
\hline $\begin{array}{c}\text { Gruppe } 3 \\
(n=24=100 \%)\end{array}$ & - & 14 & 8 & 2 \\
\hline $\begin{array}{c}\text { Gruppe } 4 \\
(n=3=100 \%)\end{array}$ & - & $(58 \%)$ & $(33 \%)$ & $(8 \%)$ \\
\hline
\end{tabular}

\subsubsection{Reanimation durch einen Notarzt}

Bei $56 \%$ der verstorbenen Kinder fand noch eine vom Notarzt durchgeführte Reanimation statt, 44\% wurden nicht mehr reanimiert.

Auf das jeweilige Geschlecht bezogen ergibt sich, dass bei $46 \%$ der verstorbenen Mädchen noch eine Reanimation versucht wurde, hingegen bei 63\% der verstorbenen Jungen (Tabelle 22).

Tabelle 22: Obduktionsfälle BMBF-Studie, Institut f. Rechtsmedizin München 19992001: Reanimation durch Notarzt

\begin{tabular}{|c|c|c|}
\cline { 2 - 3 } \multicolumn{1}{c|}{} & reanimiert & nicht reanimiert \\
\hline weiblich & 11 & 13 \\
$(n=24=100 \%)$ & $(46 \%)$ & $(54 \%)$ \\
\hline männlich & 19 & 11 \\
$(n=30=100 \%)$ & $(63 \%)$ & $(37 \%)$ \\
\hline gesamt & 30 & 24 \\
$(n=54=100 \%)$ & $(56 \%)$ & $(44 \%)$ \\
\hline
\end{tabular}




\subsection{Obduktionsbefunde}

Die in der folgenden Tabelle dargestellten Befunde der in die Studie eingeschlossenen Kinder wurden nach der Obduktion aus den Obduktionsprotokollen zusammengestellt (Tabelle 23). Petechien wurden mit einer Operations-Stereolupe beurteilt.

Tabelle 23: Obduktionsfälle BMBF-Studie, Institut f. Rechtsmedizin München 19992001: Obduktionsbefunde

\begin{tabular}{|c|c|c|c|c|c|c|c|c|c|c|c|c|c|c|c|c|c|c|c|c|}
\hline & \multicolumn{20}{|c|}{ Obduktionsbefunde } \\
\hline $\begin{array}{l}\text { Sektions- } \\
\text { Nummer }\end{array}$ & 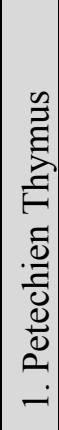 & 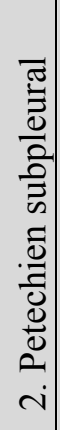 & 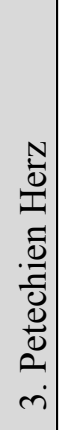 & 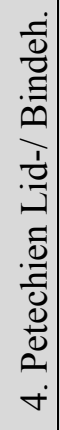 & 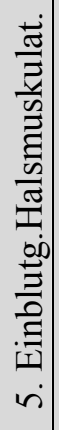 & 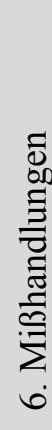 & 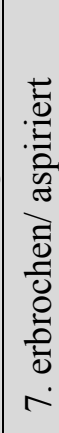 & 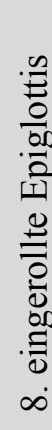 & $\begin{array}{l}0 \\
0 \\
0 \\
0 \\
3 \\
3 \\
0 \\
0 \\
0 \\
: 0 \\
0 \\
0 \\
0 \\
0\end{array}$ & 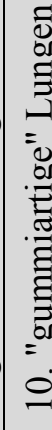 & 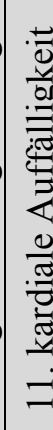 & $\begin{array}{l}0 \\
0 \\
2 \\
2\end{array}$ & & & 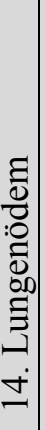 & 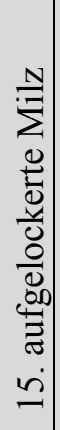 & 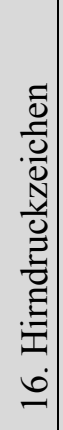 & 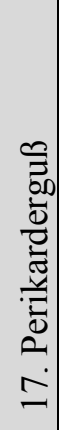 & 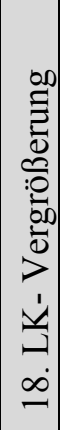 & 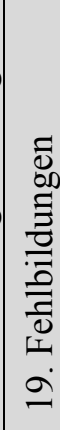 \\
\hline GS 171/99 & $\mathrm{x}$ & $\mathrm{x}$ & $\mathrm{x}$ & & & & $\mathrm{x}$ & & & & & & & & & & & & & \\
\hline GS $175 / 99$ & $\mathrm{x}$ & & & & & & $\mathrm{x}$ & & & & & & & & & $\mathrm{X}$ & $\mathrm{x}$ & & & \\
\hline GS 181/99 & $\mathrm{x}$ & $\mathrm{x}$ & $\mathrm{x}$ & $\mathrm{x}$ & & & & $\mathrm{X}$ & $\mathrm{x}$ & & & & & & & & $\mathrm{x}$ & & & \\
\hline GS 262/99 & $\mathrm{x}$ & $\mathrm{x}$ & $\mathrm{x}$ & $\mathrm{x}$ & & & & & & & & & & & & & $\mathrm{x}$ & & $\mathrm{x}$ & \\
\hline GS 284/99 & $\mathrm{x}$ & & $\mathrm{x}$ & & & & & & $\mathrm{x}$ & & & $\mathrm{x}$ & & & & & $\mathrm{x}$ & & $\mathrm{x}$ & \\
\hline GS 511/99 & $\mathrm{x}$ & $\mathrm{x}$ & & & & & & & & & & & & & & & & & $\mathrm{x}$ & \\
\hline GS 602/99 & $\mathrm{x}$ & $\mathrm{x}$ & $\mathrm{x}$ & & & & & & $\mathrm{x}$ & $\mathrm{x}$ & & $\mathrm{x}$ & & & & & $\mathrm{x}$ & & $\mathrm{x}$ & \\
\hline GS 760/99 & $\mathrm{x}$ & $\mathrm{x}$ & $\mathrm{x}$ & & & & & & $\mathrm{x}$ & $\mathrm{X}$ & & & & & & & $\mathrm{x}$ & & $\mathrm{x}$ & $\mathrm{x}$ \\
\hline GS 781/99 & $\mathrm{x}$ & $\mathrm{x}$ & & & & & & & & $\mathrm{X}$ & & & & & & & & & $\mathrm{x}$ & \\
\hline GS 852/99 & & & & $\mathrm{x}$ & & $\mathrm{x}$ & & & & & & & & & & & & & & \\
\hline GS 914/99 & $\mathrm{x}$ & $\mathrm{x}$ & $\mathrm{x}$ & & & & & & & $\mathrm{x}$ & & & & & & & $\mathrm{x}$ & & $\mathrm{x}$ & \\
\hline GS 950/99 & $\mathrm{x}$ & & $\mathrm{x}$ & & & & & & & & & & & & & $\mathrm{x}$ & $\mathrm{x}$ & & $\mathrm{x}$ & \\
\hline GS 981/99 & $\mathrm{x}$ & & $\mathrm{x}$ & & & & & & $\mathrm{x}$ & $\mathrm{X}$ & & & & & $\mathrm{x}$ & & & & & \\
\hline GS 1018/99 & $\mathrm{x}$ & $\mathrm{X}$ & $\mathrm{x}$ & & & & & $\mathrm{X}$ & $\mathrm{x}$ & & & $\mathrm{x}$ & & & $\mathrm{X}$ & & $\mathrm{x}$ & & $\mathrm{x}$ & \\
\hline GS 1079/99 & & $\mathrm{x}$ & & & & & & & & $\mathrm{X}$ & & & & & & & & & & \\
\hline GS 1080/99 & & & & & & & & & & & $\mathrm{X}$ & & & & & & & $\mathrm{x}$ & & $\mathrm{x}$ \\
\hline GS 1155/99 & & & $\mathrm{x}$ & & & & & & $\mathrm{x}$ & & & & & & & $\mathrm{x}$ & $\mathrm{x}$ & & & \\
\hline GS 1184/99 & $\mathrm{x}$ & $\mathrm{x}$ & & $\mathrm{x}$ & & & & & & $\mathrm{X}$ & & $\mathrm{x}$ & & & & $\mathrm{x}$ & & & $\mathrm{x}$ & \\
\hline GS 1195/99 & $\mathrm{x}$ & & $\mathrm{x}$ & & & & & & & $\mathrm{X}$ & & $\mathrm{x}$ & & & & $\mathrm{x}$ & $\mathrm{x}$ & & $\mathrm{x}$ & \\
\hline GS 1258/99 & $\mathrm{x}$ & & $\mathrm{x}$ & & & & & & $\mathrm{x}$ & $\mathrm{X}$ & & $\mathrm{X}$ & & & & $\mathrm{x}$ & $\mathrm{x}$ & & $\mathrm{x}$ & \\
\hline GS 1459/99 & $\mathrm{x}$ & & & & & & & & & & & $\mathrm{X}$ & & & & & & & & \\
\hline GS 1537/99 & $\mathrm{x}$ & & $\mathrm{x}$ & & & & & & & & $\mathrm{X}$ & & & & & $\mathrm{x}$ & & & & $\mathrm{x}$ \\
\hline GS 1624/99 & $\mathrm{x}$ & & & & $\mathrm{x}$ & & & $\mathrm{x}$ & $\mathrm{x}$ & $\mathrm{X}$ & & & & & $X$ & & & & & \\
\hline GS 1625/99 & $\mathrm{x}$ & & $\mathrm{x}$ & $\mathrm{X}$ & & & & & & $\mathrm{X}$ & & & & & & & & $\mathrm{x}$ & $\mathrm{x}$ & \\
\hline
\end{tabular}




\begin{tabular}{|c|c|c|c|c|c|c|c|c|c|c|c|c|c|c|c|c|c|c|c|}
\hline & 1 & 2 & 3 & 4 & 5 & 6 & 7 & 8 & 9 & 10 & 11 & 12 & 13 & 14 & 15 & 16 & 17 & 18 & 19 \\
\hline GS 1645/99 & $\mathrm{x}$ & $\mathrm{x}$ & $\mathrm{x}$ & $\mathrm{X}$ & & & & & $\mathrm{X}$ & & & $\mathrm{X}$ & & & & & & $\mathrm{X}$ & \\
\hline GS 1685/99 & $\mathrm{x}$ & $\mathrm{x}$ & $\mathrm{x}$ & & & & & & & & & & & $\mathrm{x}$ & & & & & \\
\hline GS 1821/99 & $\mathrm{x}$ & & $\mathrm{X}$ & & & & $\mathrm{x}$ & & $\mathrm{x}$ & & & $\mathrm{x}$ & & & & $\mathrm{x}$ & & & \\
\hline GS 1862/99 & $\mathrm{x}$ & & $\mathrm{x}$ & & & & & & $\mathrm{x}$ & $\mathrm{x}$ & & $\mathrm{x}$ & & & & & & & \\
\hline GS 1891/99 & $\mathrm{x}$ & & $\mathrm{x}$ & & & & $\mathrm{x}$ & & $\mathrm{x}$ & & & & & & $\mathrm{x}$ & $\mathrm{x}$ & & & \\
\hline GS 1961/99 & $\mathrm{x}$ & & & $\mathrm{X}$ & & & & & $\mathrm{x}$ & $\mathrm{X}$ & & & & & $\mathrm{X}$ & $\mathrm{x}$ & & $\mathrm{X}$ & \\
\hline GS 2133/99 & $\mathrm{x}$ & & & & & & & & & & & & & & & & & & \\
\hline GS 2139/99 & $\mathrm{x}$ & & & $\mathrm{x}$ & $\mathrm{x}$ & & $\mathrm{x}$ & & $\mathrm{x}$ & & & & & & & $\mathrm{x}$ & $\mathrm{x}$ & $\mathrm{x}$ & \\
\hline GS 2160/99 & & & & & & & & & & & & & & $\mathrm{x}$ & & & $\mathrm{x}$ & & \\
\hline GS 59/00 & $\mathrm{x}$ & $\mathrm{x}$ & $\mathrm{x}$ & & & & & & & $\mathrm{x}$ & & & & & $\mathrm{x}$ & & & & \\
\hline GS 133/00 & $\mathrm{x}$ & $\mathrm{x}$ & $\mathrm{x}$ & $\mathrm{X}$ & & & & & & & & & & & & $\mathrm{X}$ & & $\mathrm{X}$ & \\
\hline GS 161/00 & $\mathrm{x}$ & $\mathrm{x}$ & & & & & & & $\mathrm{x}$ & & & $\mathrm{x}$ & & $\mathrm{x}$ & & $\mathrm{x}$ & & $\mathrm{X}$ & \\
\hline GS 232/00 & $\mathrm{x}$ & & $\mathrm{x}$ & & & & & & $\mathrm{x}$ & & & & & & & & & & \\
\hline GS 275/00 & & & & & & & & & & & & & & & & & & & \\
\hline GS 381/00 & $\mathrm{x}$ & $\mathrm{x}$ & $\mathrm{X}$ & & & & & & & & & & & $\mathrm{X}$ & & & & $\mathrm{X}$ & \\
\hline GS 393/00 & $\mathrm{x}$ & $\mathrm{x}$ & $\mathrm{x}$ & & & & & & & & & $\mathrm{x}$ & & & & $\mathrm{x}$ & & & \\
\hline GS 413/00 & & & & & & & & & & & & & & & & $\mathrm{x}$ & & $\mathrm{x}$ & \\
\hline GS 442/00 & & & & $\mathrm{x}$ & & $\mathrm{x}$ & & & & & & & & & & & & & \\
\hline GS 500/00 & $\mathrm{x}$ & & $\mathrm{x}$ & & & & $\mathrm{X}$ & & & & & $\mathrm{x}$ & & & & & & & \\
\hline GS 759/00 & $\mathrm{x}$ & $\mathrm{x}$ & $\mathrm{x}$ & $\mathrm{x}$ & & & & & $\mathrm{x}$ & $\mathrm{x}$ & & $\mathrm{x}$ & & & & & & $\mathrm{X}$ & \\
\hline GS 824/00 & $\mathrm{x}$ & $\mathrm{x}$ & $\mathrm{x}$ & & & & & & $\mathrm{x}$ & & & & & $\mathrm{x}$ & & & & & \\
\hline GS 896/00 & $\mathrm{x}$ & $\mathrm{x}$ & $\mathrm{x}$ & & & & & & & & & $\mathrm{x}$ & & $\mathrm{x}$ & & & & $\mathrm{x}$ & \\
\hline GS 986/00 & $\mathrm{x}$ & & $\mathrm{x}$ & & & & & & & & $\mathrm{x}$ & & & & & & $\mathrm{x}$ & $\mathrm{X}$ & \\
\hline GS 1015/00 & $\mathrm{x}$ & & $\mathrm{X}$ & & & & & & $\mathrm{x}$ & $\mathrm{X}$ & & & & & & $\mathrm{X}$ & & $\mathrm{X}$ & \\
\hline GS 1069/00 & & & & & & & & & & $\mathrm{x}$ & $\mathrm{x}$ & & & & & & & & $\mathrm{x}$ \\
\hline GS 1134/00 & & & & $\mathrm{x}$ & & & & & & $\mathrm{X}$ & & & & & & $\mathrm{x}$ & & & \\
\hline GS $1331 / 00$ & $\mathrm{x}$ & $\mathrm{x}$ & $\mathrm{X}$ & $\mathrm{X}$ & & & & & & & & & & & & & & & \\
\hline GS $1333 / 00$ & $\mathrm{x}$ & & & & & & & & & & & $\mathrm{x}$ & & $\mathrm{x}$ & $\mathrm{X}$ & & & & \\
\hline GS 1371/00 & $\mathrm{x}$ & $\mathrm{x}$ & & $\mathrm{x}$ & & & & & & & & & & & & $\mathrm{x}$ & & $\mathrm{X}$ & \\
\hline GS 1687/00 & $\mathrm{x}$ & & & & $\mathrm{x}$ & & & & $\mathrm{x}$ & & & & & $\mathrm{x}$ & $\mathrm{x}$ & & & $\mathrm{x}$ & \\
\hline GS 1768/00 & $\mathrm{x}$ & $\mathrm{x}$ & & & & & & & $\mathrm{x}$ & & & & & $\mathrm{x}$ & & $\mathrm{X}$ & & & \\
\hline GS $1789 / 00$ & & & & & & & & $\mathrm{X}$ & & & & & & & & $\mathrm{x}$ & & $\mathrm{x}$ & $\mathrm{x}$ \\
\hline GS 1814/00 & & & & & & & $\mathrm{X}$ & & & & $\mathrm{x}$ & & & & & & $\mathrm{x}$ & & \\
\hline GS 1857/00 & $\mathrm{x}$ & $\mathrm{x}$ & $\mathrm{x}$ & $\mathrm{x}$ & $\mathrm{x}$ & & $\mathrm{X}$ & & & & & & & & & & & $\mathrm{x}$ & \\
\hline GS 2116/00 & $\mathrm{x}$ & & & $\mathrm{x}$ & & & & & & & & & & $\mathrm{X}$ & $\mathrm{X}$ & & & & \\
\hline GS 2180/00 & $\mathrm{x}$ & & $\mathrm{x}$ & $\mathrm{x}$ & & & & & & & & & & $\mathrm{x}$ & & & & $\mathrm{x}$ & \\
\hline GS 2201/00 & $\mathrm{x}$ & & $\mathrm{x}$ & & & & & & $\mathrm{x}$ & & & & & $\mathrm{x}$ & $\mathrm{x}$ & & & $\mathrm{X}$ & \\
\hline GS 138/01 & $\mathrm{x}$ & & & & & & & & $\mathrm{x}$ & & & $\mathrm{x}$ & & & & & & $\mathrm{x}$ & \\
\hline GS 145/01 & & & & $\mathrm{X}$ & & $\mathrm{X}$ & & & & $\mathrm{X}$ & & & & & & $\mathrm{x}$ & & & \\
\hline GS 309/01 & $\mathrm{x}$ & $\mathrm{x}$ & $\mathrm{x}$ & & & & & & & & & $\mathrm{x}$ & & & & & & & \\
\hline GS 381/01 & & & & $\mathrm{X}$ & & $\mathrm{X}$ & & & & & & & & & & $\mathrm{X}$ & & $\mathrm{x}$ & \\
\hline GS 493/01 & $\mathrm{x}$ & & $\mathrm{x}$ & $\mathrm{x}$ & $\mathrm{X}$ & & & & & $\mathrm{X}$ & & & & & & & & $\mathrm{x}$ & \\
\hline GS 565/01 & $\mathrm{x}$ & $\mathrm{x}$ & $\mathrm{x}$ & & $\mathrm{x}$ & & $\mathrm{X}$ & & & & & & & & & & & & \\
\hline GS 576/01 & $\mathrm{x}$ & & & & & & & & & & & & & & & & & $\mathrm{x}$ & \\
\hline GS 801/01 & & & & $\mathrm{X}$ & & & & & $\mathrm{X}$ & & & $\mathrm{X}$ & & & & & & $\mathrm{x}$ & \\
\hline
\end{tabular}




\begin{tabular}{|c|c|c|c|c|c|c|c|c|c|c|c|c|c|c|c|c|c|c|c|}
\hline & 1 & 2 & 3 & 4 & 5 & 6 & 7 & 8 & 9 & 10 & 11 & 12 & 13 & 14 & 15 & 16 & 17 & 18 & 19 \\
\hline GS 889/01 & $\mathrm{x}$ & & $\mathrm{x}$ & & & & & & $\mathrm{x}$ & & $\mathrm{x}$ & & & & & $\mathrm{x}$ & & $\mathrm{x}$ & \\
\hline GS 961/01 & & $\mathrm{x}$ & $\mathrm{x}$ & $\mathrm{x}$ & & & & & & & & & & & & & & & \\
\hline GS 980/01 & $\mathrm{x}$ & $\mathrm{x}$ & $\mathrm{x}$ & & & & & & $\mathrm{x}$ & $\mathrm{x}$ & & & & & & $\mathrm{x}$ & & $\mathrm{x}$ & \\
\hline GS 1096/01 & $\mathrm{x}$ & $\mathrm{x}$ & $\mathrm{x}$ & & & & & & & & & & & & & $\mathrm{x}$ & & & \\
\hline GS 1171/01 & & & $\mathrm{x}$ & & & & $\mathrm{x}$ & & & & $\mathrm{x}$ & & & & $\mathrm{x}$ & & & & \\
\hline GS 1355/01 & & & & & & & & & & & & & & $\mathrm{x}$ & $\mathrm{x}$ & & & $\mathrm{x}$ & \\
\hline GS 1456/01 & & & & & & & & & & & & & & & & & & $\mathrm{x}$ & \\
\hline GS 1554/01 & $\mathrm{x}$ & & & $\mathrm{x}$ & & & & & & $\mathrm{x}$ & & & & & & & & & \\
\hline GS $1746 / 01$ & $\mathrm{x}$ & & & & & & & & $\mathrm{x}$ & & & & & & & & & & \\
\hline GS 1923/01 & & & & & & & & & $\mathrm{x}$ & & & & & & & & & & \\
\hline
\end{tabular}

Hier alle erhobenen Befunde, jedoch geordnet nach Häufigkeit des Auftretens (Tabelle 24).

Tabelle 24: Obduktionsfälle BMBF-Studie, Institut f. Rechtsmedizin München 19992001: Häufigkeit der einzelnen Obduktionsbefunde

\begin{tabular}{|l|c|}
\hline \multicolumn{1}{|c|}{ Befund } & Anzahl (n) \\
\hline Petechien: Thymus & $60(75 \%)$ \\
\hline Petechien: am Herz(-beutel) & $44(55 \%)$ \\
\hline Vergrößerte Hals-/ Gekröselymphknoten & $39(49 \%)$ \\
\hline Petechien: subpleural & $31(39 \%)$ \\
\hline Hirndruck & $31(39 \%)$ \\
\hline gerötete Luftwege & $29(36 \%)$ \\
\hline Petechien: Lid- und/ oder Bindehäute & $24(30 \%)$ \\
\hline "gummiartige" Beschaffenheit der Lungen & $22(28 \%)$ \\
\hline Mittelohrentzündung eitrig/ serös & $20(25 \%)$ \\
\hline aufgelockerte Milz & $17(21 \%)$ \\
\hline Lungenödem & $17(21 \%)$ \\
\hline agonales Erbrechen / Aspiration & $10(13 \%)$ \\
\hline pathologische kardiale Befunde & $7(9 \%)$ \\
\hline Einblutungen in die Halsmuskulatur & $6(8 \%)$ \\
\hline Perikarderguß & $6(8 \%)$ \\
\hline Fehlbildungen & $5(6 \%)$ \\
\hline Anzeichen für Mißhandlungen & $4(5 \%)$ \\
\hline eingerollte Epiglottis & $4(5 \%)$ \\
\hline nekrotisierende Enteritis & $1(1 \%)$ \\
\hline
\end{tabular}




\subsection{Histologie}

Das Mikroskopieren der Präparate erfolgte „blind“, das heißt es lagen keine Obduktionsprotokolle oder anamnestische Angaben vor, um die Befunderhebung nicht zu beeinflussen. Um die bei den Kindern mikroskopisch erhobenen relevanten Befunde vergleichen zu können, wurden diese tabellarisiert. Insgesamt waren 15 verschiedene pathologische Befunde von Bedeutung.

Es gab sechs Kinder, bei denen sich histologisch gar keine Auffälligkeiten nachweisen ließen, alle anderen wiesen krankhafte Befunde auf, die unterschiedlich stark ausgeprägt waren. So gab es beispielsweise leichte interstitielle Pneumonien, aber auch schwerere Formen. Diese Abstufungen wurden bei der individuellen Fallkategorisierung in Kapitel 3.8. berücksichtigt, jedoch nicht in der folgenden Tabelle (Tabelle 25).

Tabelle 25: : Obduktionsfälle BMBF-Studie, Institut f. Rechtsmedizin München 19992001: Histologische Befunde

\begin{tabular}{|c|c|c|c|c|c|c|c|c|c|c|c|c|c|c|c|}
\hline $\begin{array}{l}\text { Sektions- } \\
\text { Nummer }\end{array}$ & 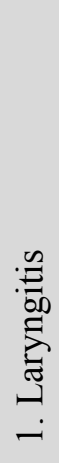 & 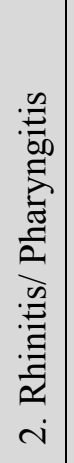 & 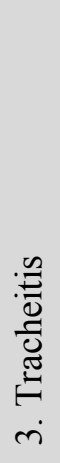 & 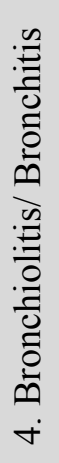 & 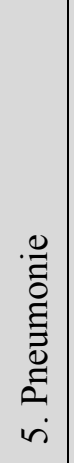 & 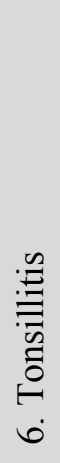 & 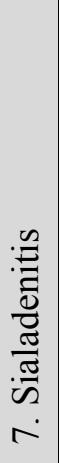 & 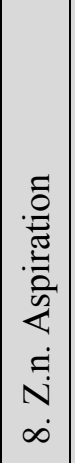 & 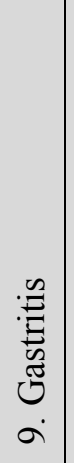 & 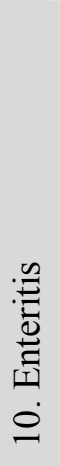 & \begin{tabular}{l}
$: \mathscr{\Xi}$ \\
\hdashline $\bar{\Xi}$ \\
$\mathscr{1}$ \\
$\doteq$ \\
$\beth$
\end{tabular} & 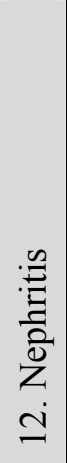 & 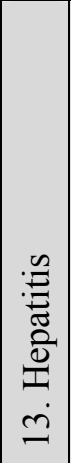 & 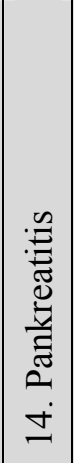 & 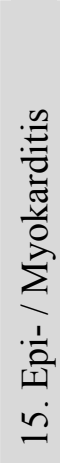 \\
\hline GS 171/99 & $\mathrm{x}$ & $\mathrm{X}$ & & & & & & & & $\mathrm{x}$ & $\mathrm{x}$ & & & & \\
\hline GS 175/99 & $\mathrm{x}$ & $\mathrm{x}$ & $\mathrm{x}$ & & & & & & & & & & & & \\
\hline GS 181/99 & $\mathrm{x}$ & $\mathrm{x}$ & $\mathrm{x}$ & & & & & & & & & & & & \\
\hline GS 262/99 & $\mathrm{x}$ & & $\mathrm{x}$ & $\mathrm{x}$ & & $\mathrm{x}$ & & & & $\mathrm{x}$ & $\mathrm{x}$ & & & & \\
\hline GS 284/99 & $\mathrm{x}$ & $\mathrm{x}$ & $\mathrm{x}$ & & & & & & & $\mathrm{x}$ & $\mathrm{x}$ & $\mathrm{x}$ & & & \\
\hline GS 511/99 & $\mathrm{x}$ & $\mathrm{x}$ & $\mathrm{x}$ & & & & & & & $\mathrm{x}$ & & & & & \\
\hline GS 602/99 & $\mathrm{x}$ & $\mathrm{x}$ & $\mathrm{x}$ & & & $\mathrm{x}$ & & & & $\mathrm{x}$ & & & & & \\
\hline GS 760/99 & & & & & & & & & & & & & & & \\
\hline GS 781/99 & & & & & & & & & & $\mathrm{x}$ & $\mathrm{x}$ & & & & \\
\hline GS 852/99 & & & & $\mathrm{x}$ & $\mathrm{x}$ & & & & & & & & & & \\
\hline GS 914/99 & & & $\mathrm{x}$ & & & & & & & & & & & & \\
\hline GS 950/99 & & & $\mathrm{x}$ & $\mathrm{x}$ & $\mathrm{x}$ & & $\mathrm{x}$ & & & $\mathrm{x}$ & $\mathrm{x}$ & & & & \\
\hline GS 981/99 & & $\mathrm{x}$ & & & $\mathrm{x}$ & & & & & $\mathrm{x}$ & $\mathrm{x}$ & & & & \\
\hline GS 1018/99 & $\mathrm{x}$ & & & $\mathrm{x}$ & $\mathrm{x}$ & & $\mathrm{x}$ & & & $\mathrm{x}$ & $\mathrm{x}$ & & & & \\
\hline GS 1079/99 & $\mathrm{x}$ & & $\mathrm{x}$ & & & & & & & & & & & & \\
\hline
\end{tabular}




\begin{tabular}{|c|c|c|c|c|c|c|c|c|c|c|c|c|c|c|c|}
\hline & 1 & 2 & 3 & 4 & 5 & 6 & 7 & 8 & 9 & 11 & 12 & 14 & 16 & 17 & 19 \\
\hline GS 1080/99 & & & & & & & & & & & & & & & \\
\hline GS 1155/99 & & & & & & & & $\mathrm{x}$ & & & & & & & \\
\hline GS 1184/99 & & & $\mathrm{x}$ & $\mathrm{x}$ & & $x$ & & & & & & & & & \\
\hline GS 1195/99 & & & & & & & & & & & & & & & \\
\hline GS 1258/99 & $\mathrm{x}$ & & $\mathrm{x}$ & & & $\mathrm{x}$ & & & & & & & & & \\
\hline GS 1459/99 & $\mathrm{x}$ & & $\mathrm{X}$ & & & & & & & & & & & & \\
\hline GS 1537/99 & $\mathrm{x}$ & $\mathrm{x}$ & $\mathrm{x}$ & & & & & & & $\mathrm{x}$ & & & & & \\
\hline GS 1624/99 & $\mathrm{x}$ & $\mathrm{x}$ & $\mathrm{x}$ & $\mathrm{x}$ & $\mathrm{x}$ & & & & & & & & & & \\
\hline GS 1625/99 & & & & & & & & & & $\mathrm{x}$ & $\mathrm{x}$ & & & & \\
\hline GS 1645/99 & & & $\mathrm{X}$ & $\mathrm{x}$ & & & & & & & & & & & \\
\hline GS $1685 / 99$ & $\mathrm{x}$ & & & & & & & & & & & & & & \\
\hline GS 1821/99 & & $\mathrm{x}$ & $\mathrm{x}$ & $\mathrm{x}$ & $\mathrm{x}$ & & $\mathrm{x}$ & $\mathrm{x}$ & & $\mathrm{x}$ & $\mathrm{x}$ & & $\mathrm{x}$ & & \\
\hline GS 1862/99 & $\mathrm{x}$ & & & & & & & & & & & & & & \\
\hline GS 1891/99 & $\mathrm{x}$ & & $\mathrm{x}$ & & & & & & & & & & & & \\
\hline GS 1961/99 & $\mathrm{x}$ & & & $\mathrm{x}$ & & & & & & & & & & & \\
\hline GS 2133/99 & & & & & & & & & & $\mathrm{x}$ & & & & & \\
\hline GS 2139/99 & $\mathrm{x}$ & $\mathrm{x}$ & $\mathrm{x}$ & & & & & $\mathrm{x}$ & & $\mathrm{x}$ & & & & & \\
\hline GS 2160/99 & & & & & & & & $\mathrm{x}$ & & & & & & & \\
\hline GS 59/00 & & & & & & & $\mathrm{x}$ & $\mathrm{x}$ & & & & & & & \\
\hline GS $133 / 00$ & $\mathrm{x}$ & & $\mathrm{x}$ & $\mathrm{x}$ & $\mathrm{x}$ & $\mathrm{x}$ & & & & & & & & & \\
\hline GS 161/00 & $\mathrm{x}$ & $\mathrm{x}$ & $\mathrm{x}$ & $\mathrm{x}$ & & & & & & & & & & & \\
\hline GS 232/00 & $\mathrm{x}$ & $\mathrm{x}$ & $\mathrm{x}$ & $\mathrm{x}$ & $\mathrm{x}$ & & & & & $\mathrm{x}$ & & & & & \\
\hline GS 275/00 & & & & & & & & & & & & & & & \\
\hline GS 381/00 & $\mathrm{x}$ & $\mathrm{x}$ & $\mathrm{x}$ & $\mathrm{x}$ & & & $\mathrm{x}$ & & & & & & & & \\
\hline GS 393/00 & $\mathrm{x}$ & & $\mathrm{X}$ & $\mathrm{x}$ & & $\mathrm{X}$ & & & $\mathrm{X}$ & $\mathrm{x}$ & & & & & \\
\hline GS 413/00 & & & & & & & & & & & & & & & \\
\hline GS 442/00 & & & & & & & & & & & $\mathrm{x}$ & & $\mathrm{x}$ & $\mathrm{x}$ & \\
\hline GS 500/00 & & & & & $\mathrm{x}$ & & & & & $\mathrm{x}$ & $\mathrm{x}$ & & & & \\
\hline GS 759/00 & $\mathrm{x}$ & $\mathrm{x}$ & $\mathrm{x}$ & $\mathrm{x}$ & $\mathrm{x}$ & & & & $\mathrm{x}$ & $\mathrm{x}$ & & & & & \\
\hline GS 824/00 & & $\mathrm{x}$ & & $\mathrm{x}$ & $\mathrm{x}$ & & & & & & & & $\mathrm{x}$ & & \\
\hline GS 896/00 & & & & & & & & & & & & & & & \\
\hline GS 986/00 & $\mathrm{x}$ & $\mathrm{x}$ & $\mathrm{x}$ & & $\mathrm{x}$ & & & & & & & & $\mathrm{x}$ & & \\
\hline GS $1015 / 00$ & & & $\mathrm{x}$ & $\mathrm{x}$ & $\mathrm{x}$ & & & & & & & & & & \\
\hline GS 1069/00 & $\mathrm{x}$ & & $\mathrm{X}$ & & & & & & & & $\mathrm{x}$ & & $\mathrm{x}$ & & \\
\hline GS 1134/00 & $\mathrm{x}$ & & & & & $\mathrm{x}$ & & & & & $\mathrm{x}$ & & $\mathrm{x}$ & & \\
\hline GS $1331 / 00$ & & & & & $\mathrm{x}$ & & $\mathrm{x}$ & & & $\mathrm{x}$ & & & $\mathrm{x}$ & & \\
\hline GS $1333 / 00$ & & & & & $\mathrm{x}$ & & $\mathrm{x}$ & & & & $\mathrm{x}$ & & & & \\
\hline GS 1687/00 & $\mathrm{x}$ & & $\mathrm{X}$ & & & & & & & $\mathrm{x}$ & & & & & \\
\hline GS 1768/00 & $\mathrm{x}$ & & & & $\mathrm{x}$ & $\mathrm{x}$ & & & $\mathrm{X}$ & $\mathrm{x}$ & & & $\mathrm{x}$ & & \\
\hline GS $1789 / 00$ & & & & & $\mathrm{x}$ & & & & & & & $\mathrm{x}$ & $\mathrm{x}$ & & $\mathrm{x}$ \\
\hline GS $1814 / 00$ & & & & & $\mathrm{x}$ & $\mathrm{x}$ & & & & $\mathrm{x}$ & & & & & \\
\hline GS $1857 / 00$ & $\mathrm{x}$ & & $\mathrm{x}$ & $\mathrm{x}$ & & $\mathrm{x}$ & & & & & & & $\mathrm{x}$ & & \\
\hline GS $2116 / 00$ & & & & & & & $\mathrm{x}$ & $\mathrm{x}$ & & & & & & & \\
\hline GS $2180 / 00$ & $\mathrm{x}$ & $\mathrm{x}$ & $\mathrm{x}$ & & & & & & & $\mathrm{x}$ & & & & & \\
\hline GS $2201 / 00$ & $\mathrm{x}$ & & & & & & & & & & & & $\mathrm{x}$ & & \\
\hline
\end{tabular}




\begin{tabular}{|c|c|c|c|c|c|c|c|c|c|c|c|c|c|c|c|}
\hline & 1 & 2 & 3 & 4 & 5 & 6 & 7 & 8 & 9 & 11 & 12 & 14 & 16 & 17 & 19 \\
\hline GS $138 / 01$ & $\mathrm{x}$ & & $\mathrm{x}$ & $\mathrm{x}$ & & $\mathrm{X}$ & $\mathrm{x}$ & & & & & & & & \\
\hline GS 145/01 & $\mathrm{x}$ & & $\mathrm{x}$ & & & & $\mathrm{x}$ & & & & & & & $\mathrm{x}$ & \\
\hline GS 309/01 & & & $\mathrm{x}$ & $\mathrm{x}$ & & & & & & $\mathrm{x}$ & & & & & \\
\hline GS 381/01 & & & & & & & & & & $\mathrm{x}$ & & & & & \\
\hline GS 493/01 & & & & & & & $\mathrm{x}$ & & & & & & $\mathrm{x}$ & & \\
\hline GS 565/01 & $\mathrm{x}$ & & & & & & & & & & & & & & \\
\hline GS 576/01 & $\mathrm{x}$ & & $\mathrm{x}$ & & $\mathrm{x}$ & $\mathrm{x}$ & & & & & & & & & \\
\hline GS 801/01 & $\mathrm{x}$ & $\mathrm{X}$ & $\mathrm{x}$ & & & & & & & & & & & & \\
\hline GS 889/01 & $\mathrm{x}$ & & $\mathrm{x}$ & & & $\mathrm{x}$ & $\mathrm{X}$ & & & $\mathrm{x}$ & $\mathrm{x}$ & & & & \\
\hline GS 961/01 & & & & & & $\mathrm{x}$ & & & & & & & & & \\
\hline GS 980/01 & & $\mathrm{x}$ & $\mathrm{x}$ & & & & & & & & & & & & \\
\hline GS 1096/01 & & $\mathrm{x}$ & & & & & & & & & $\mathrm{x}$ & & & & \\
\hline GS 1154/01 & $\mathrm{x}$ & $\mathrm{x}$ & $\mathrm{x}$ & & & & $\mathrm{X}$ & & & $\mathrm{X}$ & $\mathrm{x}$ & & & & $\mathrm{x}$ \\
\hline GS $1171 / 01$ & & $\mathrm{x}$ & & & & & & & & & $\mathrm{x}$ & & & & \\
\hline GS 1355/01 & $\mathrm{x}$ & & & & $\mathrm{x}$ & $\mathrm{x}$ & & $\mathrm{x}$ & & & & & & & \\
\hline GS $1456 / 01$ & $\mathrm{x}$ & & & & & & & & & $\mathrm{X}$ & & & & & \\
\hline GS 1554/01 & $\mathrm{X}$ & & & & $\mathrm{X}$ & & & & & & & & & & \\
\hline GS 1746/01 & $\mathrm{x}$ & $\mathrm{x}$ & $\mathrm{x}$ & & & $\mathrm{x}$ & & & & & & & & & \\
\hline GS 1923/01 & $\mathrm{x}$ & $\mathrm{x}$ & $\mathrm{x}$ & & & $\mathrm{X}$ & & & & & $\mathrm{x}$ & & & & $\mathrm{x}$ \\
\hline
\end{tabular}

Eine Laryngitis fand sich bei 55\% der untersuchten Kinder, es folgte die Tracheitis mit 50\% und die Enteritis, die bei 35\% der Kinder nachweisbar war. Im allgemeinen handelte es sich bis auf relativ wenige Ausnahmen um lymphozytäre Entzündungsreaktionen, Granulozyten fanden sich nur selten, wenn überhaupt dann in Kombination mit einer um ein vielfaches höheren Anzahl Lymphozyten, und nur in den Präparaten der Lunge und der Leber.

Insgesamt ergaben sich folgende Häufigkeiten der einzelnen pathologischen Befunde, geordnet nach deren Häufigkeit (Tabelle 26). 
Tabelle 26: Obduktionsfälle BMBF-Studie, Institut f. Rechtsmedizin München 19992001: Histologische Befunde geordnet nach deren Häufigkeit

\begin{tabular}{|l|c|}
\hline \multicolumn{1}{|c|}{ Befunde } & Häufigkeit der einzelnen Befunde \\
\hline Laryngitis & $44(55 \%)$ \\
\hline Tracheitis & $40(50 \%)$ \\
\hline Enteritis & $28(35 \%)$ \\
\hline Rhinitis/ Pharyngitis & $25(31 \%)$ \\
\hline Pneumonie & $21(26 \%)$ \\
\hline Bronchiolitis/ Bronchitis & $20(25 \%)$ \\
\hline Kolitis & $19(24 \%)$ \\
\hline Tonsillitis & $17(21 \%)$ \\
\hline Sialadenitis & $13(16 \%)$ \\
\hline Hepatitis & $12(15 \%)$ \\
\hline Z.n. Aspiration & $7(9 \%)$ \\
\hline Gastritis & $3(4 \%)$ \\
\hline Epikarditis/ Myokarditis & $3(4 \%)$ \\
\hline Nephritis & $2(3 \%)$ \\
\hline Pankreatitis & $2(3 \%)$ \\
\hline
\end{tabular}

\subsection{Bakteriologie}

Zur bakteriologischen Untersuchung auf pathogene Keime wurden Proben von Nasopharynx, Trachea, Leptomeninx, Milz, Lunge, Herzblut, eine Stuhlprobe und eine abgebundene Dünndarmschlinge asserviert und zur Untersuchung weitergeleitet.

Es wurden aufgrund der Eilbedürftigkeit auch die Kinder untersucht, deren Eltern nicht in eine Studienteilnahme eingewilligt hatten.

Insgesamt fanden sich 29 positive Keimnachweise, also bei 32\% der verstorbenen Kinder. In drei Fällen waren sogar zwei verschieden Keime in relevanter Konzentration nachweisbar. Das Keimspektrum beschränkte sich dabei auf fünf Erregertypen. Es fanden sich folgende pathogene Keime (Tabelle 27). 
Tabelle 27: Obduktionsfälle BMBF-Studie, Institut f. Rechtsmedizin München 19992001: Pathogene Keime der bakteriologischen Untersuchung

\begin{tabular}{|c|c|c|c|c|c|}
\cline { 2 - 6 } \multicolumn{1}{c|}{} & $\begin{array}{c}\text { Staphylo- } \\
\text { kokkus aureus }\end{array}$ & $\begin{array}{c}\text { Streptokokkus } \\
\text { pneumoniae }\end{array}$ & $\begin{array}{c}\text { andere Strep- } \\
\text { tokokkenarten }\end{array}$ & $\begin{array}{c}\text { Klebsiella } \\
\text { pneumoniae }\end{array}$ & $\begin{array}{c}\text { Neisseria } \\
\text { meningitidis }\end{array}$ \\
\hline $\begin{array}{c}\text { weiblich } \\
n=38=100 \%)\end{array}$ & $\begin{array}{c}4 \\
(11 \%)\end{array}$ & - & $\begin{array}{c}4 \\
(11 \%)\end{array}$ & - & - \\
\hline männlich & 9 & 2 & 5 & 2 & 2 \\
$(n=52=100 \%)$ & $(17 \%)$ & $(4 \%)$ & $(10 \%)$ & $(4 \%)$ & $(4 \%)$ \\
\hline $\begin{array}{c}\text { gesamt } \\
(n=90=100 \%)\end{array}$ & $\begin{array}{c}13 \\
(14 \%)\end{array}$ & $\begin{array}{c}2 \\
(2 \%)\end{array}$ & $\begin{array}{c}9 \\
(10 \%)\end{array}$ & $\begin{array}{c}2 \\
(2 \%)\end{array}$ & $(2 \%)$ \\
\hline
\end{tabular}

Fälle mit zweifach positivem Erregernachweis (Tabelle 28):

Tabelle 28: Obduktionsfälle BMBF-Studie, Institut f. Rechtsmedizin München 19992001: Fälle mit mehrfachem Keimnachweis

\begin{tabular}{|c|c|c|}
\hline Fall: & 1. Erreger & 2. Erreger \\
\hline GS-Nr. 1768/00 (männl.) & Streptokokken & Neisseria meningitidis \\
\hline GS-Nr. 565/01 (männl.) & Staphylokokkus aureus & Streptokokken \\
\hline GS-Nr. 1154/01 (weibl.) & Staphylokokkus aureus & Streptokokken \\
\hline
\end{tabular}

\subsection{Virologie}

Die bei der Obduktion asservierten Proben wurden zur virologischen Untersuchung an die Studienzentrale verschickt. Von dort erhielten wir eine Rückmeldung über positive Virennachweise. Allerdings wurden von der Studienzentrale nur Stuhlproben und Trachealabstriche untersucht. Wir erhielten Untersuchungsbefunde von allen 80 Kindern, unter denen sich folgende positive Virennachweise fanden (Tabellen 29 und 30). 
Tabelle 29: Obduktionsfälle BMBF-Studie, Institut f. Rechtsmedizin München 19992001: Virusnachweis in den Trachealabstrichen

\begin{tabular}{|c|c|c|c|c|}
\cline { 2 - 5 } \multicolumn{1}{c|}{} & \multicolumn{5}{c|}{ Virus } \\
\hline GS-Nr. & $\begin{array}{c}\text { Parainfluenza- } \\
\text { Viren }\end{array}$ & RS-Viren & Adenoviren & $\begin{array}{c}\text { Zytomegalie- } \\
\text { Viren }\end{array}$ \\
\hline $171 / 99$ & - & - & - & + \\
\hline $914 / 99$ & - & - & - & + \\
\hline $950 / 99$ & + & - & - & + \\
\hline $1258 / 99$ & + & - & - & - \\
\hline $1624 / 99$ & - & - & - & + \\
\hline $1645 / 99$ & - & - & + & - \\
\hline $1891 / 99$ & - & - & + & - \\
\hline $759 / 00$ & + & - & - & + \\
\hline $1857 / 00$ & - & - & - & + \\
\hline $145 / 01$ & - & - & - & + \\
\hline $309 / 01$ & - & + & - & + \\
\hline $381 / 01$ & - & - & - & + \\
\hline $493 / 01$ & - & - & - & + \\
\hline $1154 / 01$ & - & - & - & + \\
\hline $1355 / 01$ & - & - & - & + \\
\hline $1923 / 01$ & - & - & - & + \\
\hline
\end{tabular}

Tabelle 30: Obduktionsfälle BMBF-Studie, Institut f. Rechtsmedizin München 19992001: Virusnachweis in den Stuhlproben bei Untersuchung auf Entero-, Adeno- und Rota-Viren

\begin{tabular}{|c|c|c|c|}
\cline { 2 - 4 } \multicolumn{1}{c|}{} & \multicolumn{3}{c|}{ Virus } \\
\hline GS-Nr. & Enteroviren & Adenoviren & Rotaviren \\
\hline $1184 / 99$ & - & + & - \\
\hline $1645 / 99$ & + & - & - \\
\hline $1891 / 99$ & - & + & - \\
\hline
\end{tabular}

In den Stuhlproben waren insgesamt nur drei positive Virennachweise vorhanden. Bei Untersuchung der Trachealabstriche wurden bei insgesamt 18 Kindern (22,5\%) Viren nachgewiesen. Insgesamt, bei Zusammenfassung der Befunde von Trachealabstrich und Stuhlprobe, wiesen 20 Kinder eine Virusinfektion auf (25\%). Drei Kinder wiesen eine Doppelinfektion (ZMV- und RS-Viren; ZMV- und Parainfluenzaviren, Adeno- und Enteroviren, Influenza- und HHV-6-Viren) auf. Bei 15\% Prozent der Kinder konnte das Zytomegalievirus nachgewiesen werden. 


\subsection{Toxikologie}

Bei den verstorbenen Kindern wurde eine toxikologische Untersuchung durchgeführt. Im Rahmen der Obduktion wurden für die toxikologische Untersuchung von jedem Säugling Herzblut, Liquor, Blut aus der Oberschenkelvene, Urin, Augenkammerwasser, Gallenblase, Teile der Leber, je eine halbe Niere, Mageninhalt, Teile der Lunge und Unterhautfettgewebe asserviert. Es wurden die Proben von 66 Kindern untersucht, und zwar primär auf Benzodiazepine, Opiate, Trizyklische Antidepressiva, Amphetamine,

Kokain, Tetrahydrocannabinol, Methadon, Lysergsäugediäthylamid und Kohlenmonoxid.

In sechs Fällen erfolgte ein positiver Nachweis für bestimmte Pharmaka (Tabelle 31).

Tabelle 31: Obduktionsfälle BMBF-Studie, Institut f. Rechtsmedizin München 19992001: Nachweis von Pharmaka

\begin{tabular}{|l|l|}
\hline \multicolumn{1}{|c|}{ Sektions-Nummmer } & \multicolumn{1}{c|}{ nachweisbare Substanz } \\
\hline GS 175/99 & Lidocain (Spur) \\
\hline GS 2160/99 & Morphin 0,002 mg\% \\
\hline GS 381/00 & Carbamazepin (Spur) \\
\hline GS 1789/00 & Paracetamol 7,5mg/l \\
\hline GS 1456/01 & Morphin (wenig) \\
\hline GS 1923/01 & $\begin{array}{l}\text { Phenobarbital 44,9 mg/l; Phenytoin } 14,45 \\
\text { mg/l; Thiopental 3,9 mg/l; Diazepam } 0,225 \\
\text { mg/l }\end{array}$ \\
\hline
\end{tabular}

Das Kind mit bekannter nicht natürlicher Todesart, welches an einer KohlenmonoxidIntoxikation verstarb, wies einen CO- Wert von $41,75 \%$ auf, ansonsten lagen alle COWerte im Normbereich.

\subsection{Ethanolbestimmung und Begleitstoffanalyse}

Begleitstoffe sind -neben Ethanol- in alkoholischen Getränken vorkommende höherkettige Alkohole, in meist für das jeweilige Getränk charakteristischer Konzentration. Sie sind verantwortlich für das Aroma, aber auch für psychophysische 
Störungen, das Intoxikationsausmaß, Nystagmusveränderungen, EEG-Veränderungen und die „Katersymptomatik“ [Gilg in Soyka et al.1995].

Unsere Begleitstoffanalysen erfolgten mittels Head-Space Gaschromatographie (GC) an 51 Blutproben, wobei nicht in jedem Fall alle Begleitstoffe bestimmt werden konnten. Das durchschnittliche Alter der Kinder lag bei 9,7 Monaten, das postmortale Intervall bis zur Obduktion und damit Blutentnahme lag im Durchschnitt bei 39,2 Stunden. Bei 26 der verstorbenen Kinder waren Reanimationsmaßnahmen durchgeführt worden. Teilweise erfolgte die Untersuchung an Serum, teilweise an -oft hämolytischemVollblut. Eine Übersicht über die Befunde dieser Untersuchungen findet sich im Anhang.

Für die Beurteilung einer Alkoholaufnahme relevante Substanzen sind Ethanol, Acetaldehyd, Methanol, Aceton, Isopropanol, Propanol-1, Isobutanol, Butanol-2, Methylethylketon, Ethylacetat und Isoamylalkohole. Weitere nachweisbare flüchtige Substanzen sind beispielsweise Isopentan, HCN, Propionaldeyd, n-Hexan, Methylacetat, Dichlormethan, Halothan, Cyclohexan, Chloroform, Benzol, Toluol, Xylol oder Amylnitrit [Gilg in Soyka et al.1995].

\subsubsection{Ethanol}

Nach Umrechnung der Vollblutwerte in Serumwerte mittels eines in der Begleitstoffdiagnostik üblichen Quotienten von 1,4 [Gilg in Soyka et al.1995] ergab sich ein Mittelwert von 0,045 $\pm 0,012 \mathrm{~g} / \mathrm{l}$ beziehungsweise Promille. Die untere Grenze lag bei 0,0021 , die obere bei $0,360 \%$.

In insgesamt neun Fällen lag der Serumethanolspiegel bei mehr als 0,1\%o (Abb.11). 
Abb.11: Obduktionsfälle BMBF-Studie, Institut f. Rechtsmedizin München 1999-2001: Serumethanolspiegel bezogen auf das Alter der Kinder

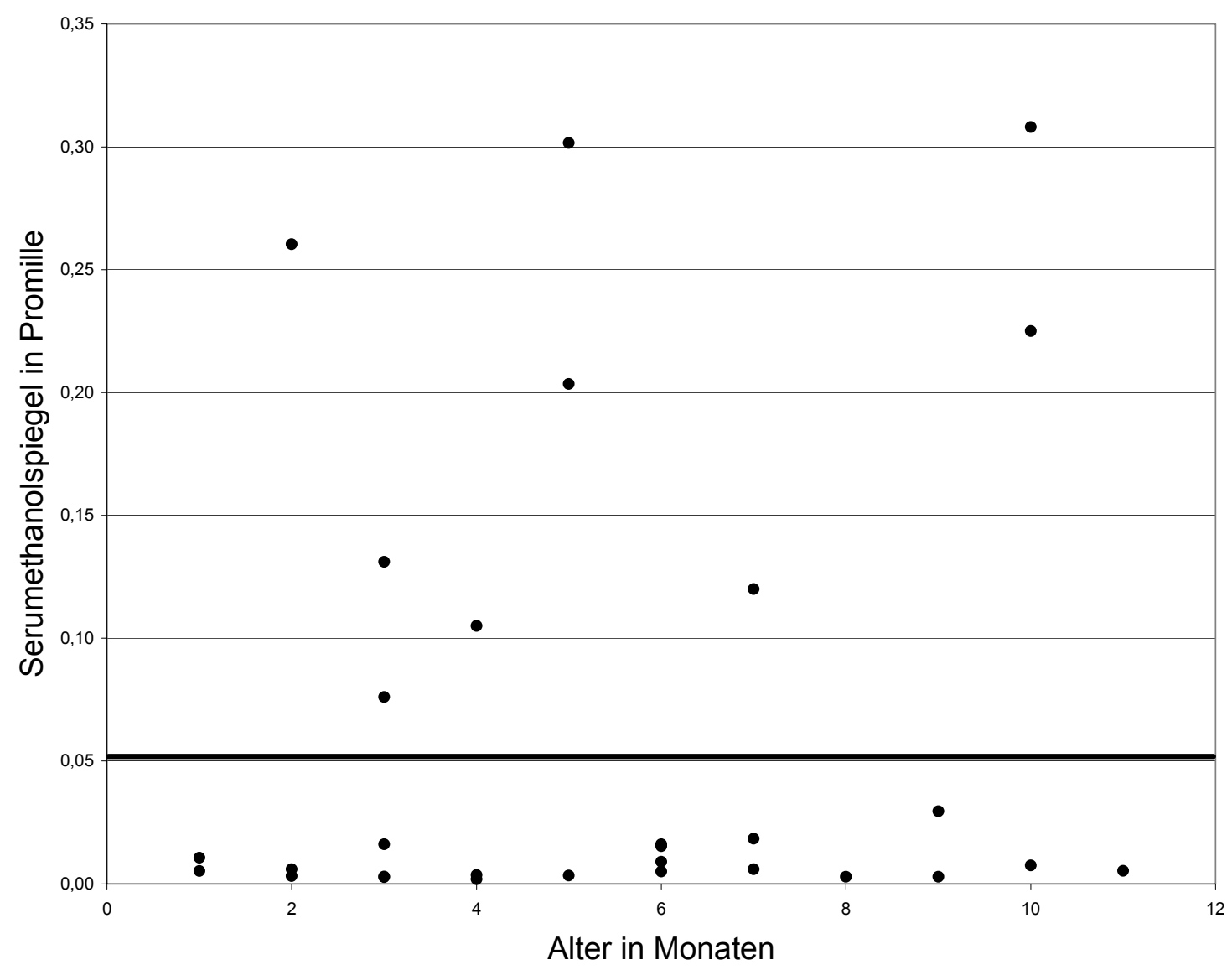

Es waren Kinder aus allen Altersgruppen betroffen. Es ergab sich kein Zusammenhang mit der Leichenliegezeit, die zwischen 8 und 95 Stunden lag. Auch durchgeführte Reanimationsmaßnahmen spielten keine Rolle.

\subsubsection{Acetaldehyd}

Ethanol wird im Carbohydrat- und Aminosäurestoffwechsel durch die Alkoholdehydrogenase (ADH) zu Acetaldehyd reduziert. Bei postmortalen Blutproben kann es im Rahmen von Hämolyse, Autolyse oder Fäulnisveränderungen zur Bildung oder Freisetzung von hämoglobin- bzw. erythrozytär gebundenem Acetaldehyd, und damit zu einer Erhöhung der Acetaldehydspiegel, kommen [Gilg in Soyka et al. 1995]. 
Der Mittelwert der von uns gemessenen Acetaldehydspiegel lag bei 2,74 $\pm 0,32 \mathrm{mg} / \mathrm{l}$, und damit etwa im Normbereich. Es ergab sich kein Zusammenhang mit dem postmortalen Intervall (Abb.12).

Abb.12: Obduktionsfälle BMBF-Studie, Institut f. Rechtsmedizin München 1999-2001: Serumacetaldehydspiegel bezogen auf die Leichenliegezeit

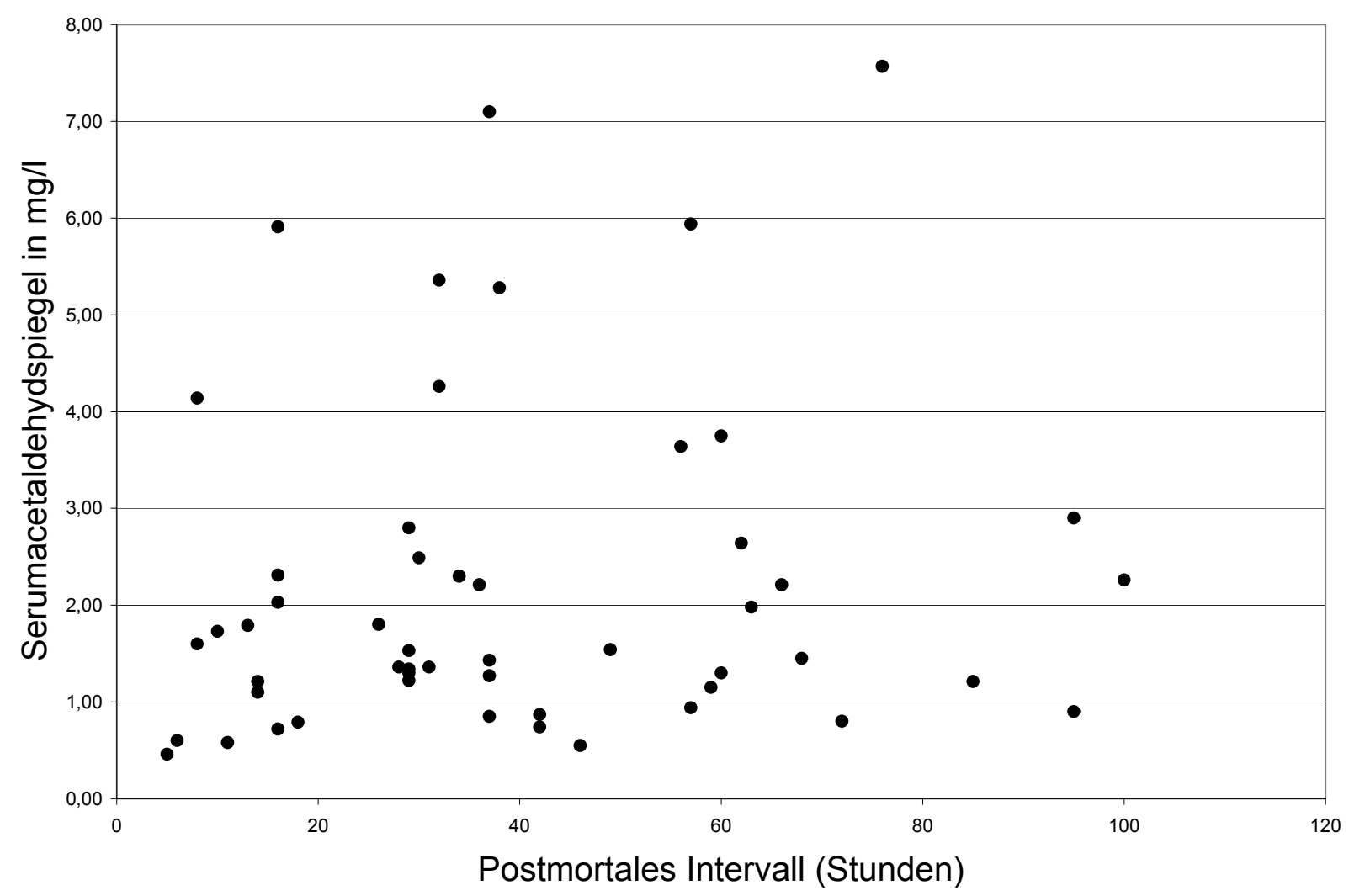

Ebenso ergab sich keine Korrelation zwischen Serumethanol- und Serumacetaldehydspiegeln (Abb.13), wie beispielsweise hohe Acetaldehydspiegel nach Ethanolabbau oder niedrige Spiegel nach Ethanolbildung [Gilg in Soyka et al. 1995]. 
Abb.13: Obduktionsfälle BMBF-Studie, Institut f. Rechtsmedizin München 1999-2001: Serumethanolspiegel in Bezug auf die Serumacetaldehydspiegel

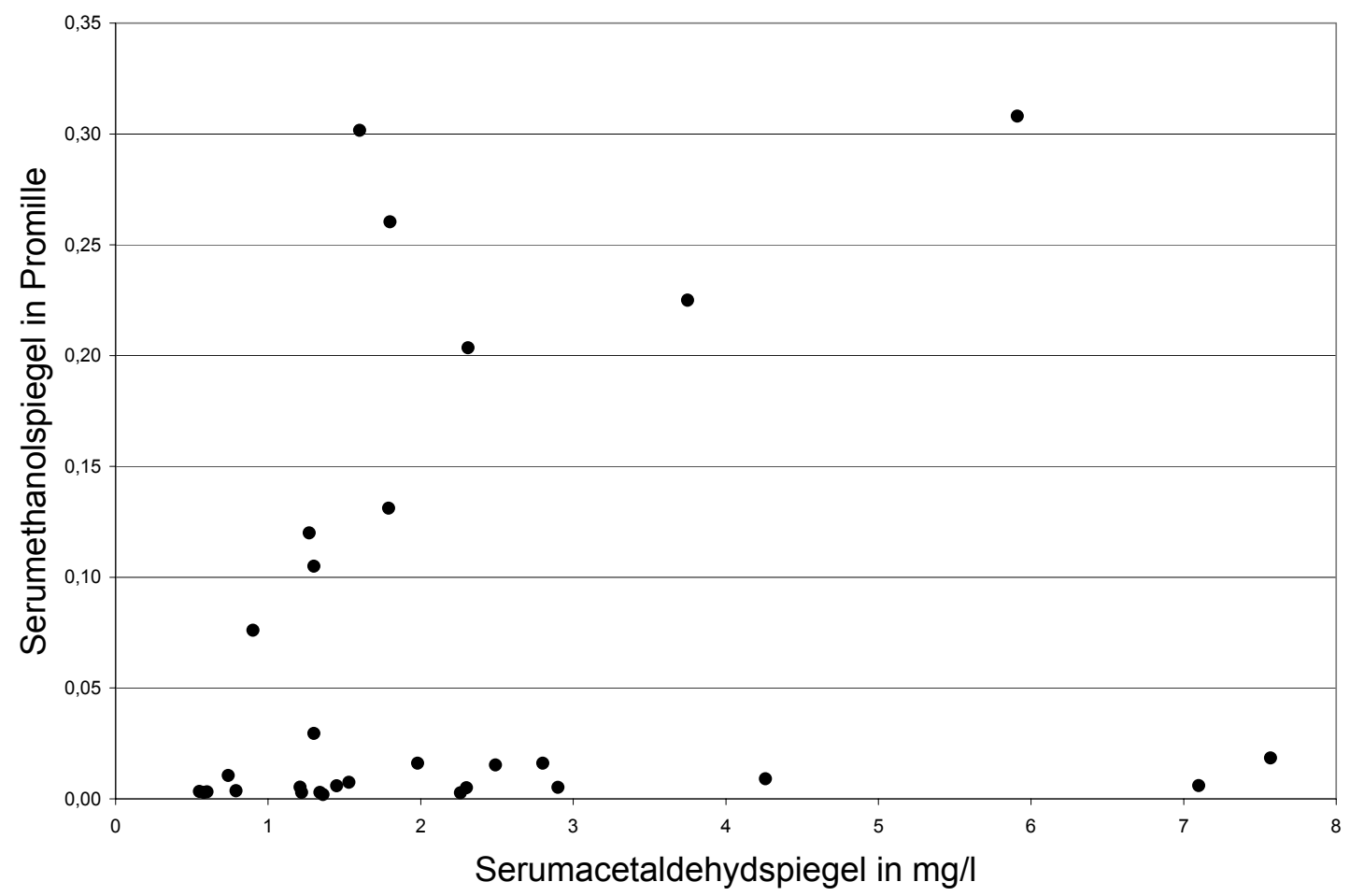

\subsubsection{Methanol}

Es fanden sich mittlere Serummethanolspiegel von 1,790 $\pm 0,128 \mathrm{mg} / \mathrm{l}$. $11 \mathrm{Kinder}$ wiesen unphysiologisch hohe Methanolspiegel [Gilg in Soyka et al. 1995] von $2 \mathrm{mg} / 1$ oder mehr auf. Es waren 6 Jungen und 9 Mädchen betroffen, das Alter lag zwischen vier und elf Monaten (Abb.14). 
Abb.14: Obduktionsfälle BMBF-Studie, Institut f. Rechtsmedizin München 1999-2001: Serummethanolspiegel bezogen auf das Alter der Kinder

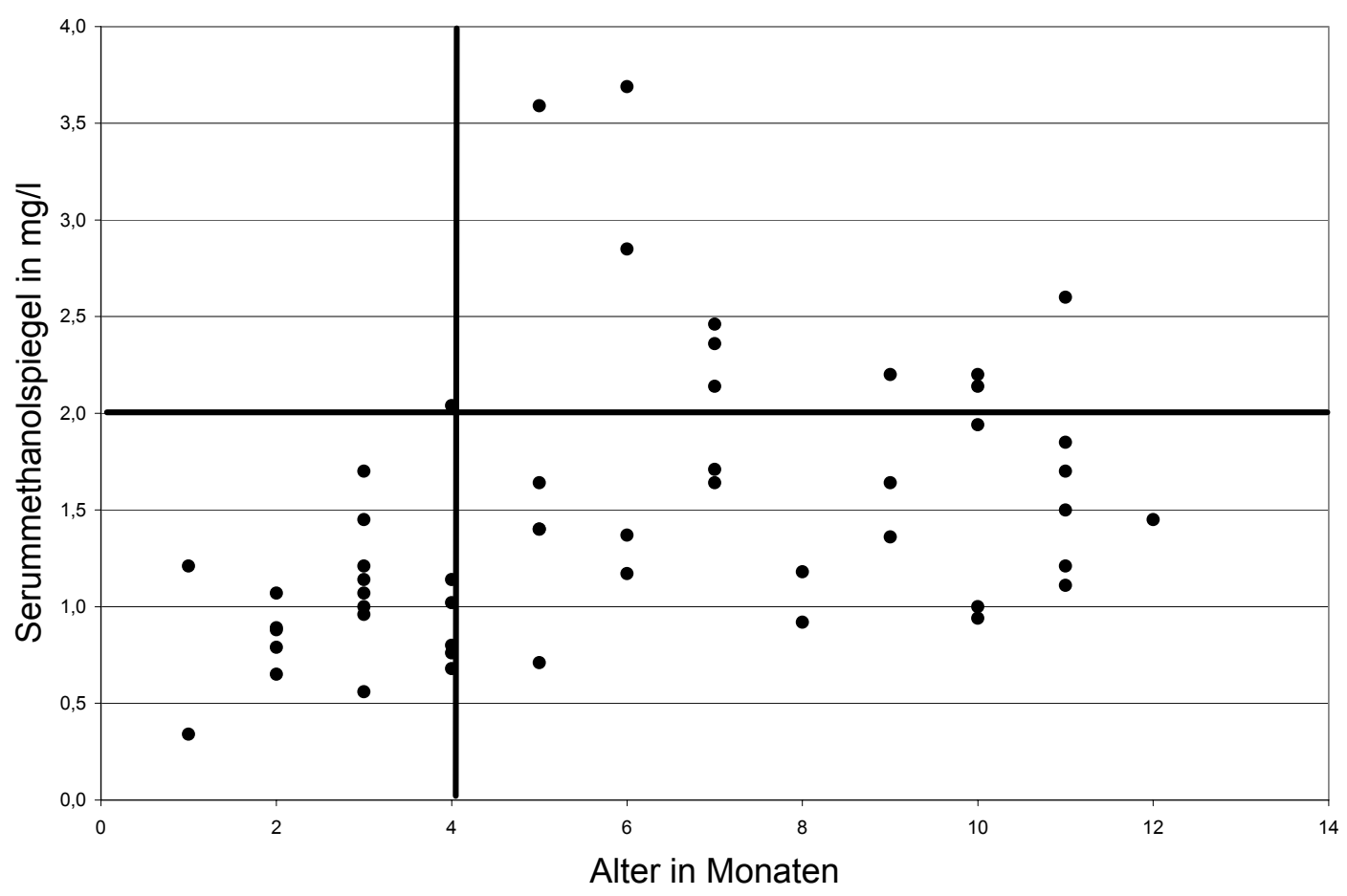

Bei Betrachtung des durchschnittlichen Serummethanolspiegels bezogen auf ein bestimmtes Lebensalter ergab sich folgende Verteilung (Abb.15). 


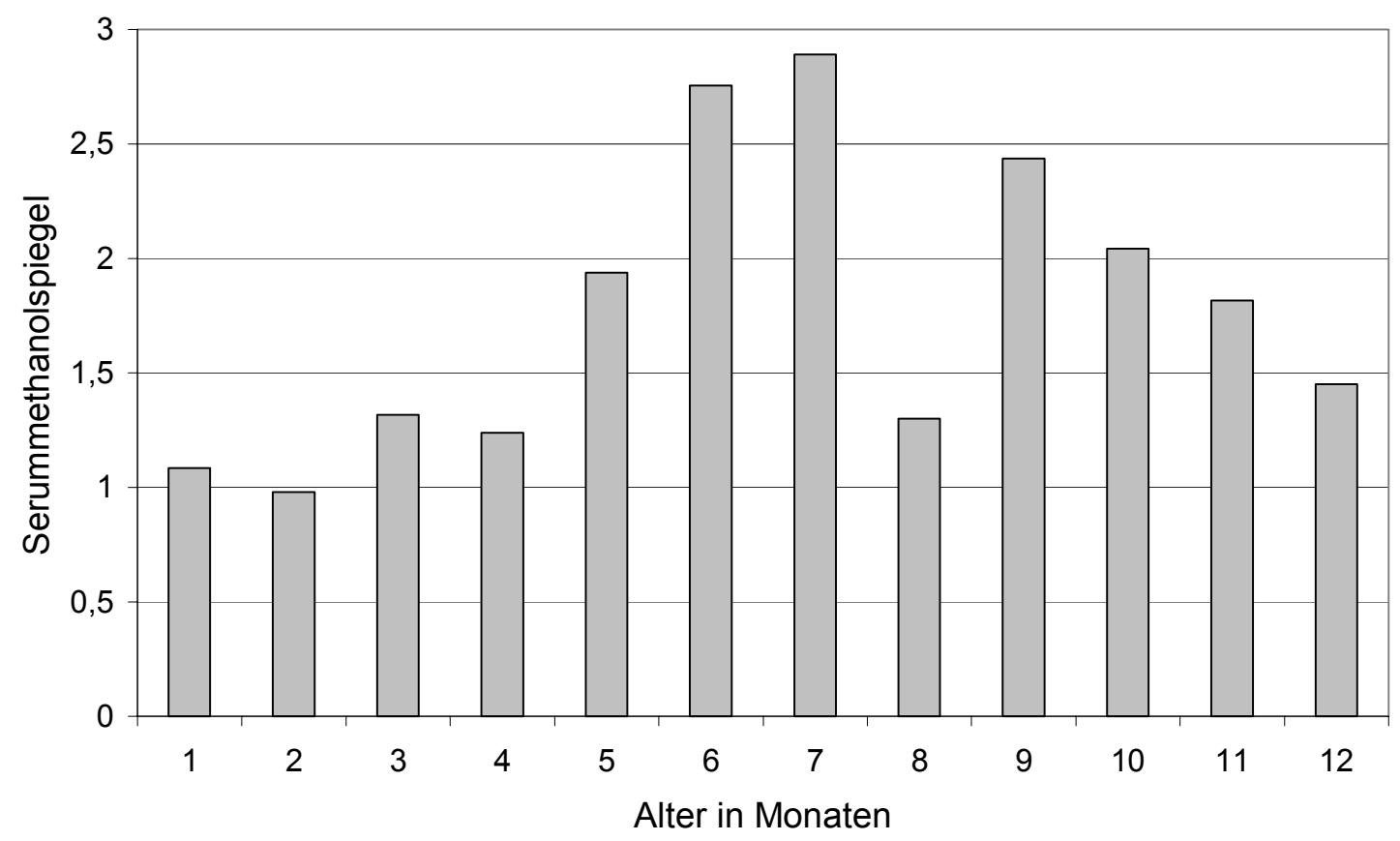

Bei Betrachtung des Methanolspiegels bezogen auf den Acetonspiegel ergab sich keine Korrelation zwischen den beiden Begleitstoffen.

\subsubsection{Aceton und Isopropanol}

Die Acetonspiegel lagen im Mittel bei 2,497 $\pm 2,488 \mathrm{mg} / \mathrm{l}$, die Grenzen lagen bei 0,37 $\mathrm{mg} / \mathrm{l}$ und 14,6 mg/l, und waren in einem Fall auffallend erhöht. Physiologische Spiegel lägen bei 0,5 bis zu $7 \mathrm{mg} / 1$ [Gilg in Soyka et al. 1995].

Für Isopropanol ergab sich ein mittlerer Spiegel von 1,3306 \pm 1,6641 mg/l, die Grenzen lagen hier bei $0,2 \mathrm{mg} / \mathrm{l}$ und 10,8 mg/l. Der physiologische Spiegel liegt hier bei bis zu 0,1 mg/l [Gilg in Soyka et al. 1995], die von uns gemessen Spiegel lagen in allen Fällen bei mehr als $0,1 \mathrm{mg} / 1$.

Eine angedeutete Korrelation zwischen den beiden biochemischen Reaktionspartnern [Gilg in Soyka et al. 1995] ließ sich anhand eines Punktediagrammes feststellen (Abb.16). 
Abb.16: Obduktionsfälle BMBF-Studie, Institut f. Rechtsmedizin München 1999-2001: Isopropanol und Aceton im Serum

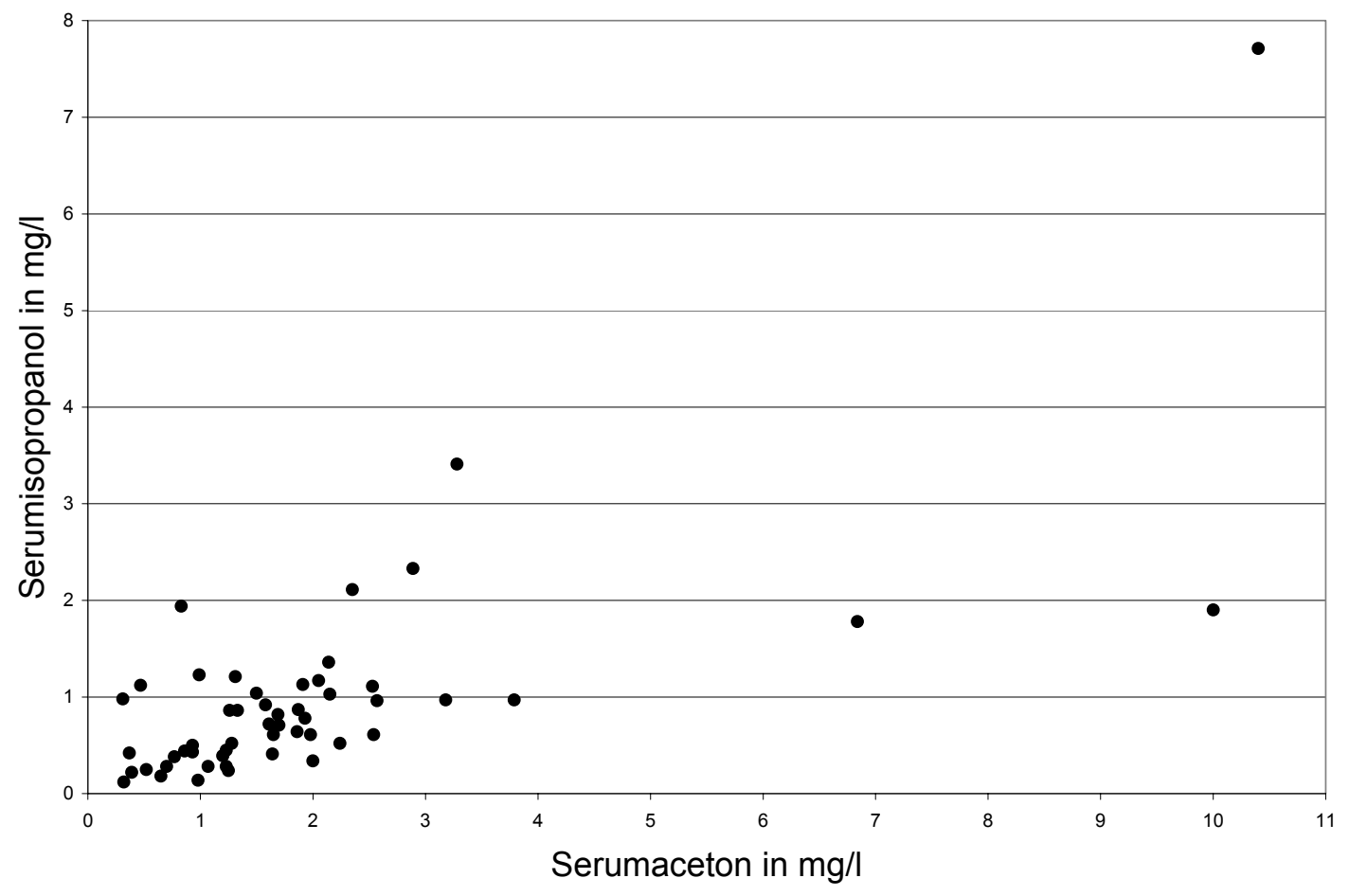

Er ergab sich kein Bezug zu einer durchgeführten Reanimation (Abb.17), zu eventueller großflächiger Hautdesinfektion, zur Leichenliegezeit, zum Alter oder Geschlecht der Kinder. 
Abb.17: Obduktionsfälle BMBF-Studie, Institut f. Rechtsmedizin München 1999-2001: Isopropanolspiegel und Reanimation

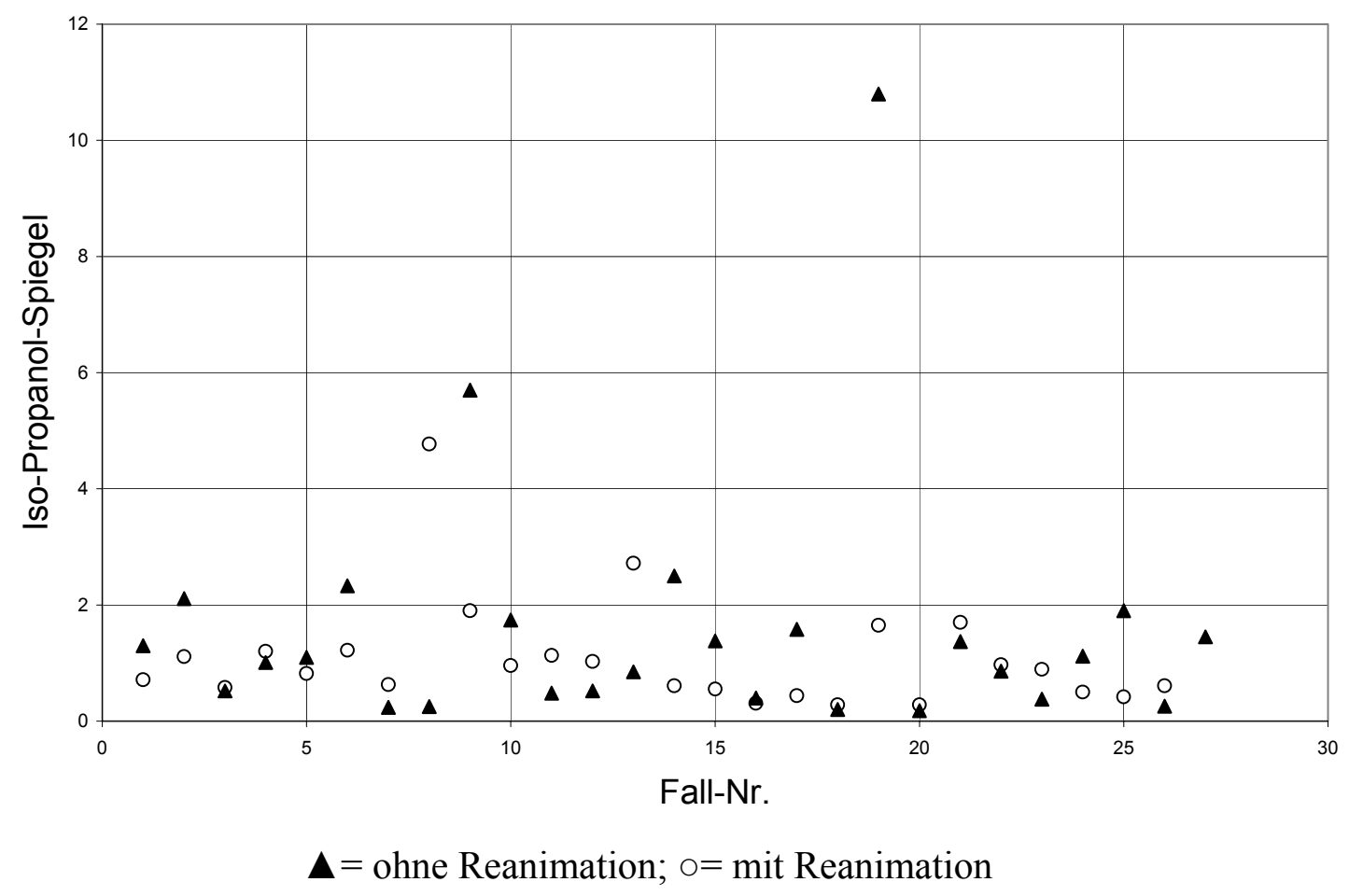

\subsection{Abschließende Kategorisierung}

Alle in die Studie eingeschlossenen Kinder wurden im Rahmen von Fallkonferenzen (mit den drei in München an der Studie beteiligten Personen) abschließend beurteilt. Dazu wurden zuerst die Ergebnisse der Obduktion betrachtet. Hinzugezogen wurden dann die histologischen Befunde unter Berücksichtigung des Schweregrads der Erkrankung und es wurde eine Einstufung in eine der Kategorien von eins bis vier vorgenommen. Schließlich wurden die Ergebnisse der bakteriologischen, virologischen und toxikologischen Untersuchungen zusammen mit der Anamnese einbezogen und die Kinder abschließend kategorisiert.

Es bleibt zu betonen, dass alle diese Kinder mit Verdacht auf Vorliegen eines Plötzlichen Säuglingstodes zur Obduktion gelangten, und erst durch unsere Untersuchungen in vielen Fällen eine andere Todesursache festgestellt werden konnte. 
Die folgende Tabelle gibt die Kategorieeinstufung der einzelnen Kinder und gegebenenfalls Besonderheiten der einzelnen Fälle wieder (Tabelle 32):

Tabelle 32: Obduktionsfälle BMBF-Studie, Institut f. Rechtsmedizin München 19992001: Kategorisierung und Auffälligkeiten

\begin{tabular}{|c|c|c|}
\hline Sektions-Nr. & Kategorie & Anmerkungen \\
\hline GS 171/99 & 2 & fragliche Blutneubildung im Schädelknochen \\
\hline GS 175/99 & 4 & Sepsis (Staph. aureus) \\
\hline GS 181/99 & 2 & - \\
\hline GS 262/99 & 4 & nekrotisierende Enterokolitis \\
\hline GS 284/99 & 2 & Pneumothorax beidseits \\
\hline GS 511/99 & 2 & - \\
\hline GS 602/99 & 2 & - \\
\hline GS 760/99 & 1 & Pierre-Robin-Syndrom, sonst unauffällig \\
\hline GS 781/99 & 2 & - \\
\hline GS 852/99 & 4 & $\begin{array}{l}\text { subdurale Blutung, etliche Weichteilblutungen im } \\
\text { Halsbereich, alte Oberarmfraktur, Tötung }\end{array}$ \\
\hline GS 914/99 & 2 & - \\
\hline GS 950/99 & 3 & - \\
\hline GS 981/99 & 2 & - \\
\hline GS $1018 / 99$ & 2 & - \\
\hline GS 1079/99 & 2 & - \\
\hline GS 1080/99 & 4 & $\begin{array}{l}\text { Williams-Beuren-Syndrom, Ergußflüssigkeit in } \\
\text { Brust- und Bauchhöhle und Perikard }\end{array}$ \\
\hline GS $1155 / 99$ & 2 & - \\
\hline GS 1184/99 & 2 & Windelekzem \\
\hline GS 1195/99 & 1 & - \\
\hline GS 1258/99 & 4 & $\begin{array}{l}\text { Sepsis (Pneumokokken), eingeblutetes } \\
\text { Nebennierenmark, Harnröhrenentzündung }\end{array}$ \\
\hline GS 1459/99 & 2 & - \\
\hline GS 1537/99 & 2 & bekannter Vorhofseptumdefekt \\
\hline GS $1624 / 9$ & 2 & - \\
\hline GS $1625 / 99$ & 2 & - \\
\hline GS $1645 / 99$ & 2 & - \\
\hline GS 1685/99 & 2 & - \\
\hline GS $1821 / 99$ & 3 & - \\
\hline GS $1862 / 99$ & 2 & - \\
\hline GS 1891/99 & 2 & beidseits zweigelappte Lungen \\
\hline GS 1961/99 & 2 & - \\
\hline GS 2133/99 & 2 & $\begin{array}{l}\text { im Harn und Liquor stark positiver Zuckertest, } \\
\text { vermutlich reanimationsbedingt }\end{array}$ \\
\hline GS 2139/99 & 2 & - \\
\hline
\end{tabular}




\begin{tabular}{|c|c|c|}
\hline Sektions-Nr. & Kategorie & Anmerkungen \\
\hline GS 2160/99 & 4 & $\begin{array}{l}\text { Ersticken durch Blutaspiration, positiver } \\
\text { Morphinnachweis, Blut im Magen }\end{array}$ \\
\hline GS 59/00 & 2 & - \\
\hline GS $133 / 00$ & 2 & bekannte abgeheilte Windpocken-Erkrankung \\
\hline GS $161 / 00$ & 2 & - \\
\hline GS 232/00 & 4 & Influenza A \\
\hline GS $275 / 00$ & 1 & $\begin{array}{l}\text { Verlauf der Vena subclavia über den Thymus } \\
\text { hinweg mit isthmusartiger Einengung }\end{array}$ \\
\hline GS 381/00 & 2 & - \\
\hline GS 393/00 & 2 & auffällig weite Aorta mit blasser Wand \\
\hline GS 413/00 & 4 & Sepsis (Streptokokken), Windeldermatitis \\
\hline GS 442/00 & 4 & $\begin{array}{l}\text { viele Petechien, alte Schädelfraktur und ältere } \\
\text { subdurale Blutung, angeblich Auffindung in } \\
\text { Rückenlage, aber Livores ventral; Tötung }\end{array}$ \\
\hline GS 500/00 & 2 & $\begin{array}{l}\text { fleckig-rötliche, fast wie eingeblutet aussehende } \\
\text { Lungen }\end{array}$ \\
\hline GS 759/00 & 3 & $\begin{array}{l}\text { Petechien an Lid-/ Bindehäuten, Augenwinkel, } \\
\text { Jochbeinregion, Vorder- und Hinterohrregion, } \\
\text { Halshaut, Nacken, Brustkorb und Oberarmen }\end{array}$ \\
\hline GS 824/99 & 3 & - \\
\hline GS 896/00 & 1 & einzelner Gallenblasenbettlymphknoten \\
\hline GS 986/00 & 4 & $\begin{array}{l}\text { durchlöcherte Membran des Foramen ovale, } \\
\text { Rechtsherzhypertrophie, verfestige Lungenober- } \\
\text { lappen, Perikarderguß }(30 \mathrm{ml})\end{array}$ \\
\hline GS $1015 / 00$ & 2 & - \\
\hline GS 1069/00 & 4 & $\begin{array}{l}\text { Williams-Beuren-Syndrom mit Myokardinfarkt, } \\
\text { maximal positiver Harnzuckertest }\end{array}$ \\
\hline GS 1134/00 & 4 & Waterhouse-Friderichsen-Syndrom \\
\hline GS 1331/00 & 2 & - \\
\hline GS $1333 / 00$ & 2 & - \\
\hline GS $1371 / 00$ & --- & $\begin{array}{l}\text { Histologie nicht mehr beurteilbar bei } \\
\text { fortgeschrittener Autolyse }\end{array}$ \\
\hline GS $1687 / 00$ & 2 & - \\
\hline GS $1768 / 00$ & 3 & - \\
\hline GS 1789/00 & 4 & $\begin{array}{l}\text { Sepsis (Streptokokken), Adrenogenitales Syndrom } \\
\text { (AGS), Mittelketten- Acyl-CoA-Dehydrogenase- } \\
\text { Defekt (MCAD) }\end{array}$ \\
\hline GS $1814 / 00$ & 4 & $\begin{array}{l}\text { Sepsis (Streptokokken), großes ausgeweitetes } \\
\text { Herz, Ergußflüssigkeit in Brust- und Bauchhöhle } \\
\text { und Perikard }\end{array}$ \\
\hline GS $1857 / 00$ & 3 & $\begin{array}{l}\text { sehr großer Schädel (Hirngewicht } 1235 \mathrm{~g} \text { ), fragliche } \\
\text { Ausweitung des rechten Herzens }\end{array}$ \\
\hline GS $2116 / 00$ & 2 & - \\
\hline GS 2180/00 & 3 & - \\
\hline GS 2201/00 & 2 & - \\
\hline GS 138/01 & 2 & - \\
\hline
\end{tabular}




\begin{tabular}{|c|c|c|}
\hline Sektions-Nr. & Kategorie & Anmerkungen \\
\hline GS 145/01 & 4 & $\begin{array}{l}\text { große subdurale und subarachnoidale Blutung } \\
\text { rechts, Sinusvenenthrombose, Tötung }\end{array}$ \\
\hline GS 309/01 & 3 & - \\
\hline GS 381/01 & 4 & $\begin{array}{l}\text { viele Petechien, Blutung in das Augenweiß rechts, } \\
\text { zwei Tage alte Rippenfrakturen, frische } \\
\text { Oberschenkel- und Oberarmfraktur, } \\
\text { Schädeldachbruchsystem rechtes Hinterhaupt, } \\
\text { Tötung (Todesursache: Ersticken) }\end{array}$ \\
\hline GS 493/01 & 2 & - \\
\hline GS 565/01 & 2 & - \\
\hline GS 576/01 & 2 & - \\
\hline GS 801/01 & 2 & $\begin{array}{l}\text { fraglich vergröberte Struktur und derbe Konsistenz } \\
\text { von Lungenanteilen wie bei } \\
\text { Bindegewebsvermehrung }\end{array}$ \\
\hline GS 889/01 & 4 & $\begin{array}{l}\text { Herpesvirus-Infektion, Bluteinatmungsbezirke in } \\
\text { der Lunge, Ausweitung des rechten Vorhofs } \\
\text { (Tricuspidalklappe: } 6 \mathrm{~cm} \text { ), Mutter: aktuell Fazialis- } \\
\text { parese, Vater: aktuell Guillan-Barré-Syndrom }\end{array}$ \\
\hline GS 961/01 & 2 & $\begin{array}{l}\text { durch Reanimation/ iatrogen bedingter Hämato- } \\
\text { thorax rechts }\end{array}$ \\
\hline GS 980/01 & 2 & - \\
\hline GS 1096/01 & 2 & offenes Foramen ovale \\
\hline GS $1154 / 01$ & 3 & $\begin{array}{l}\text { möglicherweise Sepsis (Staph. aureus und } \\
\text { Streptokokken), angetrockneter Schaumpilz vor } \\
\text { der Nase }\end{array}$ \\
\hline GS 1171/01 & 2 & $\begin{array}{l}\text { an drei Stellen nicht gedecktes Foramen ovale, } \\
\text { Rechtsherzhypertrophie, insgesamt vergrößertes } \\
\text { Herz }\end{array}$ \\
\hline GS $1355 / 01$ & 2 & - \\
\hline GS $1456 / 01$ & 2 & - \\
\hline GS $1554 / 01$ & 3 & Riesenzellpneumonie unklarer Genese \\
\hline GS $1746 / 01$ & 2 & $\begin{array}{l}\text { Ausweitung der Aorta ascendens mit } \\
\text { Narbenbildung im Bogenbereich }\end{array}$ \\
\hline GS $1923 / 01$ & 4 & Myokarditis \\
\hline
\end{tabular}

Nur 5\% der Kinder konnten in Kategorie 1 eingeteilt werden, da sie in jeder Untersuchung unauffällig erschienen, und man keinen Anhalt für die Todesursache fand. Der Großteil der Kinder wurde Kategorie 2 zugeordnet (60\%). Bei 13\% konnten wir eine mögliche (Kategorie 3) und bei 23\% eine sichere Todesursache nachweisen (Kategorie 4), insgesamt also bei über einem Drittel der Kinder (Tabelle 33). 
Tabelle 33: Obduktionsfälle BMBF-Studie, Institut f. Rechtsmedizin München 19992001: Kategorisierung

\begin{tabular}{|c|c|c|c|c|}
\cline { 2 - 5 } \multicolumn{1}{c|}{} & \multicolumn{4}{c|}{ Kategorie } \\
\cline { 2 - 5 } \multicolumn{1}{c|}{} & 1 & 2 & 3 & 4 \\
\hline weiblich & 3 & 20 & 5 & 6 \\
$(n=34=100 \%)$ & $(9 \%)$ & $(59 \%)$ & $(15 \%)$ & $(18 \%)$ \\
\hline männlich & 1 & 27 & 5 & 12 \\
$(n=45=100 \%)$ & $(2 \%)$ & $(60 \%)$ & $(11 \%)$ & $(27 \%)$ \\
\hline gesamt & 4 & 47 & 10 & $(23 \%)$ \\
$(n=79=100 \%)$ & $(5 \%)$ & $(60 \%)$ & $(13 \%)$ & $(8 \%)$ \\
\hline
\end{tabular}

Unter den gesicherten Todesursachen fanden sich vier Tötungen (5\%), in allen vier Fällen handelte es sich um Jungen. Drei davon waren als "battered child" anzusehen, ein Junge verstarb an einem Schütteltrauma. In sechs Fällen (8\%) ließ sich eine Sepsis als Todesursache nachweisen (vier Jungen, zwei Mädchen). Es fanden sich zwei WilliamsBeuren-Syndrome, in einem Fall mit Myokardinfarkt. Die anderen Kinder verstarben an Influenza A, Herpesvirus-Infektion (HHV-6), Blutaspiration letztlich ungeklärter Ursache bei positivem Morphinnachweis, nekrotisierender Enterokolitis und in zwei Fällen an kardialen Erkrankungen (Myokarditis) beziehungsweise Defekten. 


\section{ERGEBNISSE - SIDS IM VERGLEICH ZU NON-SIDS}

Wie im vorigen Kapitel dargestellt, waren unter allen 79 von uns untersuchten Kinder nur vier SIDS-Fälle im engeren Sinne (entspricht 5\%) vorhanden. Bei 95\% der Fälle (!) konnten pathologische Befunde nachgewiesen werden.

Wir gehen abschließend -im Übereinstimmung mit dem Vorgehen der Studienzentraledavon aus, dass die Kinder der Kategorie 2 als SIDS-Fälle im weiteren Sinne anzusehen sind: Bei ihnen fanden sich geringe pathologische Befunde, Hinweise in der Anamnese oder mikrobiologischen Untersuchung auf eine leichte Erkrankung. Jedoch handelte es sich nicht um Erkrankungen, die -zumindest nach heutigem Wissensstand- in irgendeiner Weise den Todeseintritt erklären könnten.

Im Gegensatz zu Kapitel 3, werden im Folgenden die Untersuchungsbefunde der eindeutigen SIDS-Fälle (Kategorie 1) und der Kinder aus Kategorie 2 (SIDS-Fälle im weiteren Sinne) den Untersuchungsbefunden der im Folgenden als „Non-SIDS-Fälle“ bezeichneten (Kategorie 3 und 4) gegenübergestellt, um die Differenzen später diskutieren zu können.

\subsection{Epidemiologische Untersuchungen}

\subsubsection{Geschlecht}

Unter den SIDS-Fällen fand sich ein Verhältnis von Mädchen zu Jungen von 45\% zu $55 \%$, bei den Non-SIDS-Fällen waren es 39\% zu 61\% (Tabelle 34).

Tabelle 34: Obduktionsfälle BMBF-Studie, Institut f. Rechtsmedizin München 19992001: Geschlechtsverteilung bei SIDS- und Non-SIDS-Fällen

\begin{tabular}{|c|c|c|}
\cline { 2 - 3 } \multicolumn{1}{c|}{} & weiblich & männlich \\
\hline SIDS & 23 & 28 \\
$(n=51=100 \%)$ & $(45 \%)$ & $(55 \%)$ \\
\hline Non-SIDS & 11 & 17 \\
$(n=28=100 \%)$ & $(39 \%)$ & $(61 \%)$ \\
\hline
\end{tabular}


In der Non-SIDS-Gruppe waren die Jungen noch stärker überrepräsentiert als in der SIDS-Gruppe.

\subsubsection{Alter}

Bei den SIDS-Fällen zeigten sich zwei Altersgipfel: der dritte bis fünfte und der neunte Lebensmonat.

Bei den Non-SIDS-Fällen ließ sich eine Häufung der Todesfälle im zweiten bis fünften und im zehnten Lebensmonat feststellen (Abb. 18).

Abb. 18: Obduktionsfälle BMBF-Studie, Institut f. Rechtsmedizin München 1999-2001: Altersverteilung der Non-SIDS-Fälle im Vergleich zu SIDS-Fällen

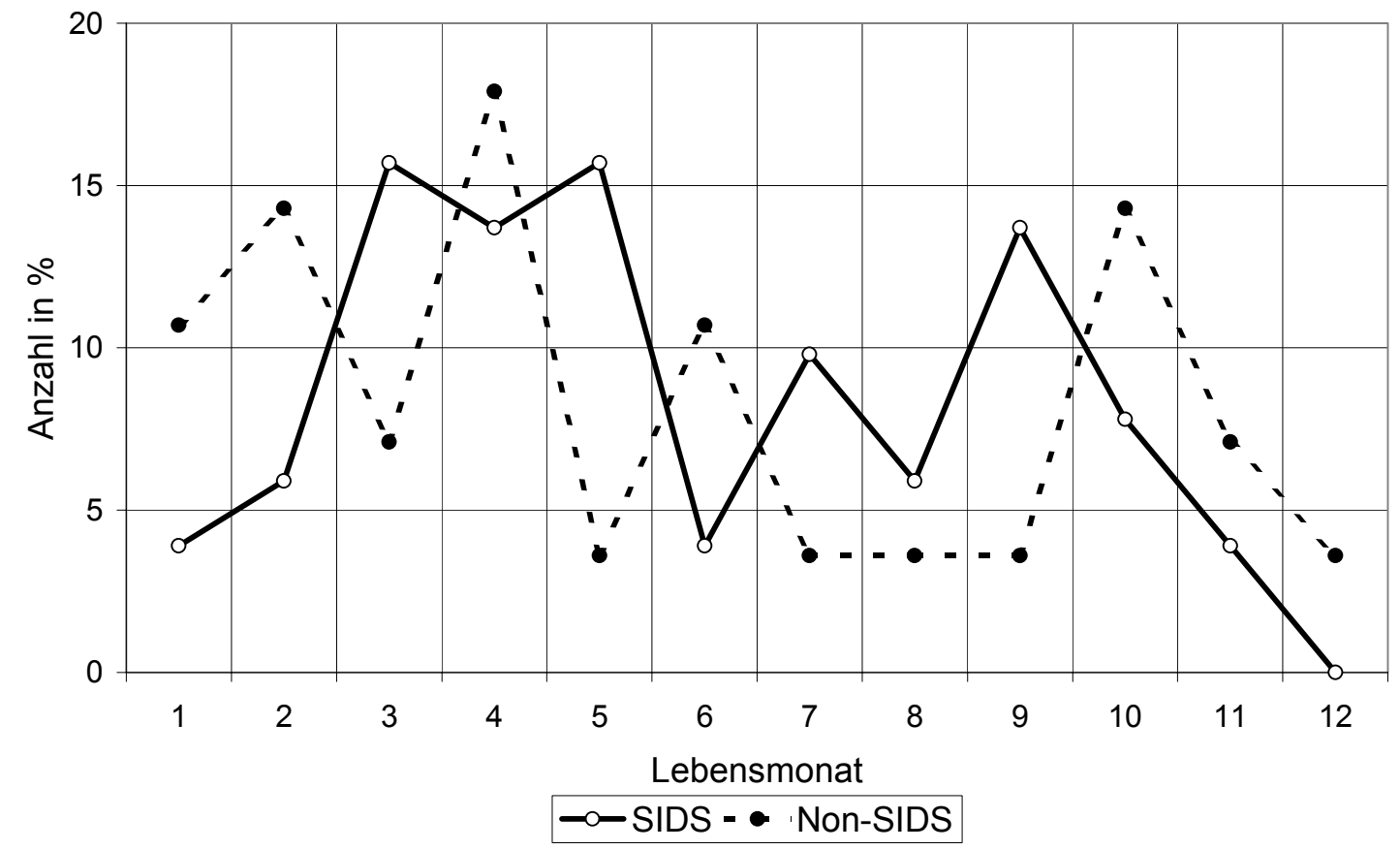

\subsubsection{Verteilung der Todesfälle auf die Wochentage}

Von den SIDS-Fällen verstarben besonders viele Kinder an Dienstagen und Samstagen. Unter den Non-SIDS-Fällen war kein Wochentag besonders häufig vertreten (Tabelle $35)$. 
Tabelle 35: Obduktionsfälle BMBF-Studie, Institut f. Rechtsmedizin München 19992001: Verteilung der Todesfälle auf die Wochentage bei SIDS- und Non-SIDS-Fällen

\begin{tabular}{|c|c|c|c|c|c|c|c|}
\cline { 2 - 7 } \multicolumn{1}{c|}{} & Mo & Di & Mi & Do & Fr & Sa & So \\
\hline $\begin{array}{c}\text { SIDS } \\
(n=50= \\
100 \%)\end{array}$ & 7 & 10 & 5 & 4 & 6 & 13 & 5 \\
$(14 \%)$ & $(20 \%)$ & $(10 \%)$ & $(8 \%)$ & $(12 \%)$ & $(26 \%)$ & $(10 \%)$ \\
\hline $\begin{array}{c}\text { Non- } \\
\text { SIDS }\end{array}$ & 4 & 2 & 5 & 5 & 4 & 5 & 3 \\
$\left(\begin{array}{c}n=28= \\
100 \%)\end{array}\right.$ & $(14 \%)$ & $(7 \%)$ & $(18 \%)$ & $(18 \%)$ & $(14 \%)$ & $(18 \%)$ & $(11 \%)$ \\
\hline
\end{tabular}

Bei Trennung der Geschlechter zeigt sich unter den SIDS-Fällen erneut, dass samstags (32\%) mehr als doppelt so viele Jungen verstarben wie an anderen Wochentagen. Bei den Mädchen war dies dienstags (32\%) der Fall. Auch bei der Gesamtschau aller SIDSFälle zeigt sich, dass der Dienstag und Samstag die häufigsten Sterbetage waren.

Bei den Non-SIDS-Fällen zeigt sich unter den Mädchen eine leichte Häufung der Todesfälle in der Wochenmitte (Mittwoch bis Freitag), bei den Jungen hebt sich der Samstag mit $24 \%$ der Todesfälle hervor.

\subsubsection{Jahreszeitliche Verteilung}

In diese Tabellen wurden, wie in Kapitel 3.1.5., nur die Fälle aus den Jahren 1999 und 2000, nicht aber aus dem unvollständigen Jahr 2001 aufgenommen.

Es verstarben die meisten SIDS-Fällen in den Monaten November bis Januar, Mai bis Juli und im September. Unter den Non-SIDS-Fällen ereigneten sich die meisten Todesfälle im Februar, April bis Juni und im Oktober (Abb. 19). 
Abb. 19: Obduktionsfälle BMBF-Studie, Institut f. Rechtsmedizin München 1999-2001: Jahreszeitliche Verteilung der SIDS- und Non-SIDS-Fälle

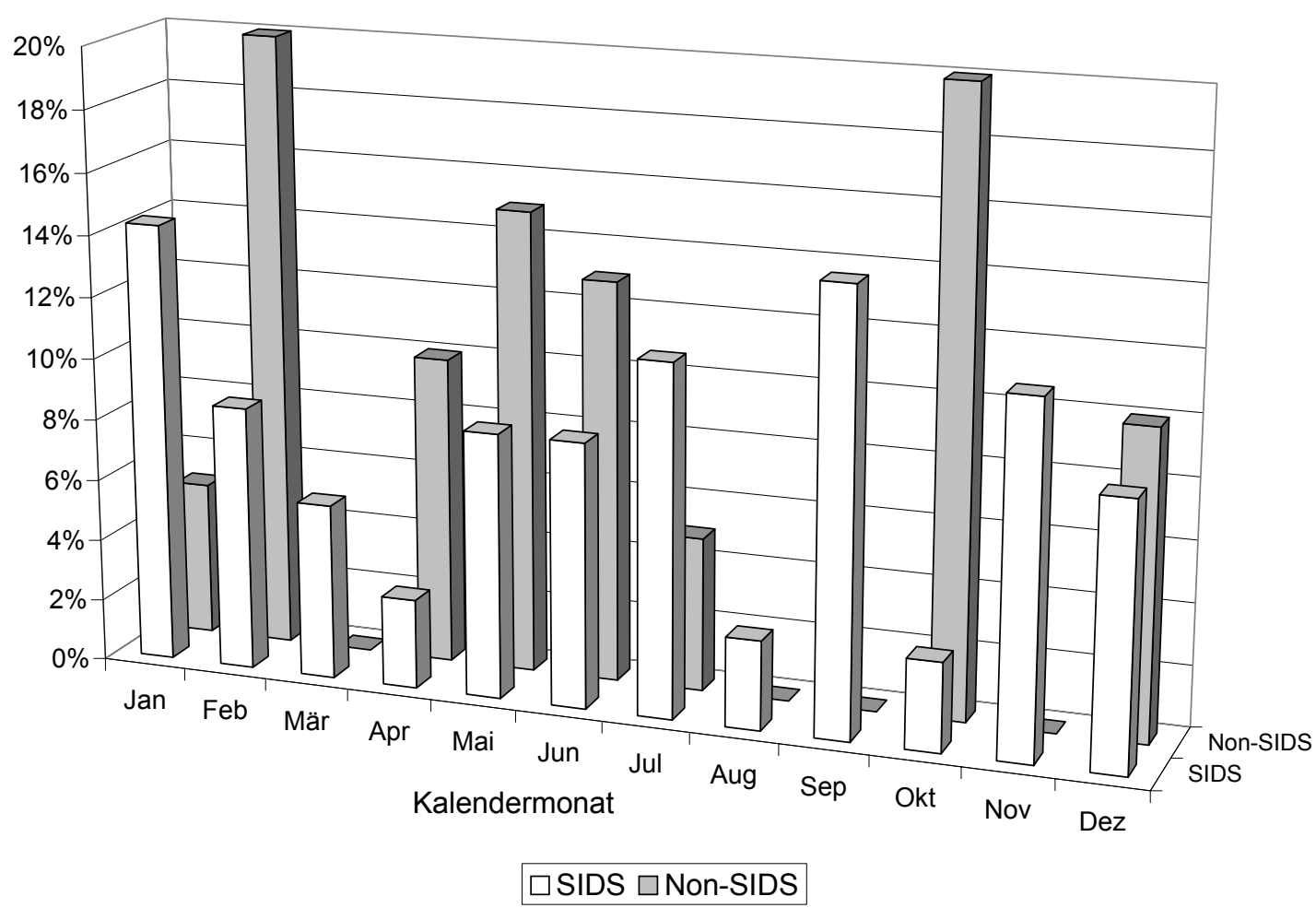

Dann wurde überprüft, ob in der SIDS- und Non-SIDS-Gruppe bestimmte Altersgruppen gehäuft in bestimmten Jahreszeiten verstarben.

Es zeigte sich, dass unter den SIDS-Fällen in den Sommermonaten eher die jüngeren Kinder verstarben (Durchschnittsalter 4,7 Monate), die älteren Kinder (Durchschnittsalter 7,1 Monat) verstarben häufig im Herbst. In der Non-SIDS-Gruppe verstarben die jüngsten Kinder (Durchschnittsalter 4,4 Monate) oft in den Wintermonaten, die ältesten mit einem durchschnittliche Alter von 6,2 Monaten eher im Herbst (Tabelle 36).

Es fanden sich hingegen keine Häufungen bestimmter Geburtsmonate unter den Todesfällen, sondern eine relativ gleichmäßige Verteilung der Geburtstage der verstorbenen Kinder über den gesamten Jahresverlauf hinweg. 
Tabelle 36: Obduktionsfälle BMBF-Studie, Institut f. Rechtsmedizin München 19992001: Jahreszeitliche Verteilung der SIDS- und Non-SIDS-Fälle, altersbezogen

\begin{tabular}{|c|c|c|c|c|}
\cline { 2 - 5 } \multicolumn{1}{c|}{} & $\begin{array}{c}\text { Frühling } \\
\text { (Mär, Apr, Mai) }\end{array}$ & $\begin{array}{c}\text { Sommer } \\
\text { (Jun, Jul, Aug) }\end{array}$ & $\begin{array}{c}\text { Herbst } \\
\text { (Sep, Okt, Nov) }\end{array}$ & $\begin{array}{c}\text { Winter } \\
\text { (Dez, Jan, Feb) }\end{array}$ \\
\hline $\begin{array}{c}\text { Durchschnitts- } \\
\text { alter SIDS }\end{array}$ & 5,8 & 4,7 & 7,1 & 5,9 \\
\hline $\begin{array}{c}\text { Durchschnitts- } \\
\text { alter Non-SIDS }\end{array}$ & 5,8 & 5,2 & 6,2 & 4,4 \\
\hline
\end{tabular}

\subsubsection{Auffindesituation}

Die SIDS-Fälle wurden zu 67\% auf dem Bauch liegend aufgefunden, die Non-SIDSFälle zu 62\%. Sowohl bei den SIDS- als auch bei den Non-SIDS-Fällen überwog die Bauchlage, es folgte die Rücken- und Seitenlage (Tabelle 37).

Tabelle 37: Obduktionsfälle BMBF-Studie, Institut f. Rechtsmedizin München 19992001: Auffindesituation bei SIDS- und Non-SIDS-Fällen

\begin{tabular}{|c|c|c|c|}
\cline { 2 - 4 } \multicolumn{1}{c|}{} & Bauch & Rücken & Seite \\
\hline SIDS & 31 & 12 & 3 \\
$(n=46=100 \%)$ & $(67 \%)$ & $(26 \%)$ & $(7 \%)$ \\
\hline Non-SIDS & 13 & 7 & 1 \\
$(n=21=100 \%)$ & $(62 \%)$ & $(33 \%)$ & $(4 \%)$ \\
\hline
\end{tabular}

Bei Untersuchung der Auffindesituation getrennt nach den Geschlechtern zeigt sich, dass unter den SIDS-Fällen 68\% der Mädchen und 67\% der Jungen in Bauchlage tot aufgefunden wurden (Tabelle 38).

Tabelle 38: Obduktionsfälle BMBF-Studie, Institut f. Rechtsmedizin München 19992001: SIDS-Auffindesituation, geschlechtsbezogen

\begin{tabular}{|c|c|c|c|}
\cline { 2 - 4 } \multicolumn{1}{c|}{} & Bauch & Rücken & Seite \\
\hline weiblich & 15 & 5 & 2 \\
$(n=22=100 \%)$ & $(68 \%)$ & $(23 \%)$ & $(9 \%)$ \\
\hline männlich & 16 & 7 & 1 \\
$(n=24=100 \%)$ & $(67 \%)$ & $(29 \%)$ & $(4 \%)$ \\
\hline
\end{tabular}


Unter den Non-SIDS-Fällen wurden 63\% der Mädchen und 62\% der Jungen in Bauchlage tot aufgefunden(Tabelle 39).

Tabelle 39: Obduktionsfälle BMBF-Studie, Institut f. Rechtsmedizin München 19992001: Non-SIDS-Auffindesituation, geschlechtsbezogen

\begin{tabular}{|c|c|c|c|}
\cline { 2 - 4 } \multicolumn{1}{c|}{} & Bauch & Rücken & Seite \\
\hline weiblich & 5 & 3 & - \\
$(\mathrm{n}=8=100 \%)$ & $(63 \%)$ & $(38 \%)$ & 1 \\
\hline männlich & 8 & 4 & $(7 \%)$ \\
\hline
\end{tabular}

\subsubsection{Schwangerschaftsdauer}

Von den SIDS-Fällen wurden 19\% nach einer Schwangerschaftsdauer von weniger als 37 Wochen geboren, unter den Non-SIDS-Fällen waren es 15\% (Tabelle 40).

Tabelle 40: Obduktionsfälle BMBF-Studie, Institut f. Rechtsmedizin München 19992001: Schwangerschaftsdauer bei SIDS- und Non-SIDS-Fällen

\begin{tabular}{|c|c|c|}
\cline { 2 - 3 } \multicolumn{1}{c|}{} & weniger als 37 Wochen & 37 und mehr Wochen \\
\hline SIDS & 6 & 26 \\
$(\mathrm{n}=32=100 \%)$ & $(19 \%)$ & $(81 \%)$ \\
\hline Non-SIDS & 3 & 17 \\
$(\mathrm{n}=20=100 \%)$ & $(15 \%)$ & $(85 \%)$ \\
\hline
\end{tabular}

Trennt man die Fälle nach Geschlechtern, so zeigt sich, dass von den SIDS-Fällen 13\% der Mädchen und 24\% der Jungen Frühgeborene waren (Tabelle 41).

Tabelle 41: Obduktionsfälle BMBF-Studie, Institut f. Rechtsmedizin München 19992001: Schwangerschaftsdauer bei SIDS-Fällen, geschlechtsbezogen

\begin{tabular}{|c|c|c|}
\cline { 2 - 3 } \multicolumn{1}{c|}{} & weniger als 37 Wochen & 37 und mehr Wochen \\
\hline weiblich & 2 & 13 \\
$(n=15=100 \%)$ & $(13 \%)$ & $(87 \%)$ \\
\hline männlich & 4 & 13 \\
$(n=17=100 \%)$ & $(24 \%)$ & $(76 \%)$ \\
\hline
\end{tabular}


Unter den Non-SIDS-Fällen gab es keine weiblichen Frühgeborenen, aber 21\% der Jungen wurden nach einer Schwangerschaftsdauer von weniger als 37 Wochen geboren (Tabelle 42).

Tabelle 42: Obduktionsfälle BMBF-Studie, Institut f. Rechtsmedizin München 19992001: Schwangerschaftsdauer bei Non-SIDS-Fällen, geschlechtsbezogen

\begin{tabular}{|c|c|c|}
\hline & weniger als 37 Wochen & 37 und mehr Wochen \\
\hline $\begin{array}{c}\text { weiblich } \\
(n=6=100 \%)\end{array}$ & 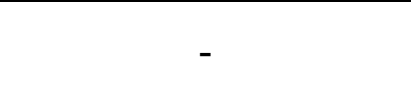 & $\begin{array}{c}6 \\
(100 \%)\end{array}$ \\
\hline $\begin{array}{c}\text { männlich } \\
(n=14=100 \%)\end{array}$ & $\begin{array}{c}3 \\
(21 \%)\end{array}$ & $\begin{array}{c}11 \\
(79 \%)\end{array}$ \\
\hline
\end{tabular}

\subsubsection{Größe und Gewicht zum Zeitpunkt der Geburt und der Obduktion}

Bei Betrachtung des Geburtsgewichtes zeigt sich, dass von den SIDS-Fällen 18\% weniger als 2500 Gramm wogen, bei den Non-SIDS-Fällen waren es 19\% (Tabelle 43).

Tabelle 43: Obduktionsfälle BMBF-Studie, Institut f. Rechtsmedizin München 19992001: Geburtsgewicht bei SIDS- und Non-SIDS-Fällen

\begin{tabular}{|c|c|c|}
\cline { 2 - 3 } \multicolumn{1}{c|}{} & $<2500 \mathrm{~g}$ & $>2500 \mathrm{~g}$ \\
\hline SIDS & 8 & 36 \\
$(n=44=100 \%)$ & $(18 \%)$ & $(82 \%)$ \\
\hline Non-SIDS & 5 & 22 \\
$(n=27=100 \%)$ & $(19 \%)$ & $(81 \%)$ \\
\hline
\end{tabular}

Das folgende Diagramm zeigt das Gewicht im Verhältnis zur Körpergröße zum Zeitpunkt der Geburt. Wir konnten vierzig SIDS-Fälle und dreiundzwanzig Non-SIDSFälle in diese Auswertung einbeziehen. Sieben SIDS-Kinder (18\%) und keines der NonSIDS-Kinder waren nach der Geburt oberhalb der 3\%-Perzentile einzuordnen, waren also für ihre Größe untergewichtig. Zu schwer hingegen waren zwei SIDS-Fälle (5\%) und drei Non-SIDS-Fälle (13\%) (Abb. 20). 
Abb. 20: Obduktionsfälle BMBF-Studie, Institut f. Rechtsmedizin München 1999-2001: Perzentilen zum Zeitpunkt der Geburt bei SIDS- und Non-SIDS-Fällen

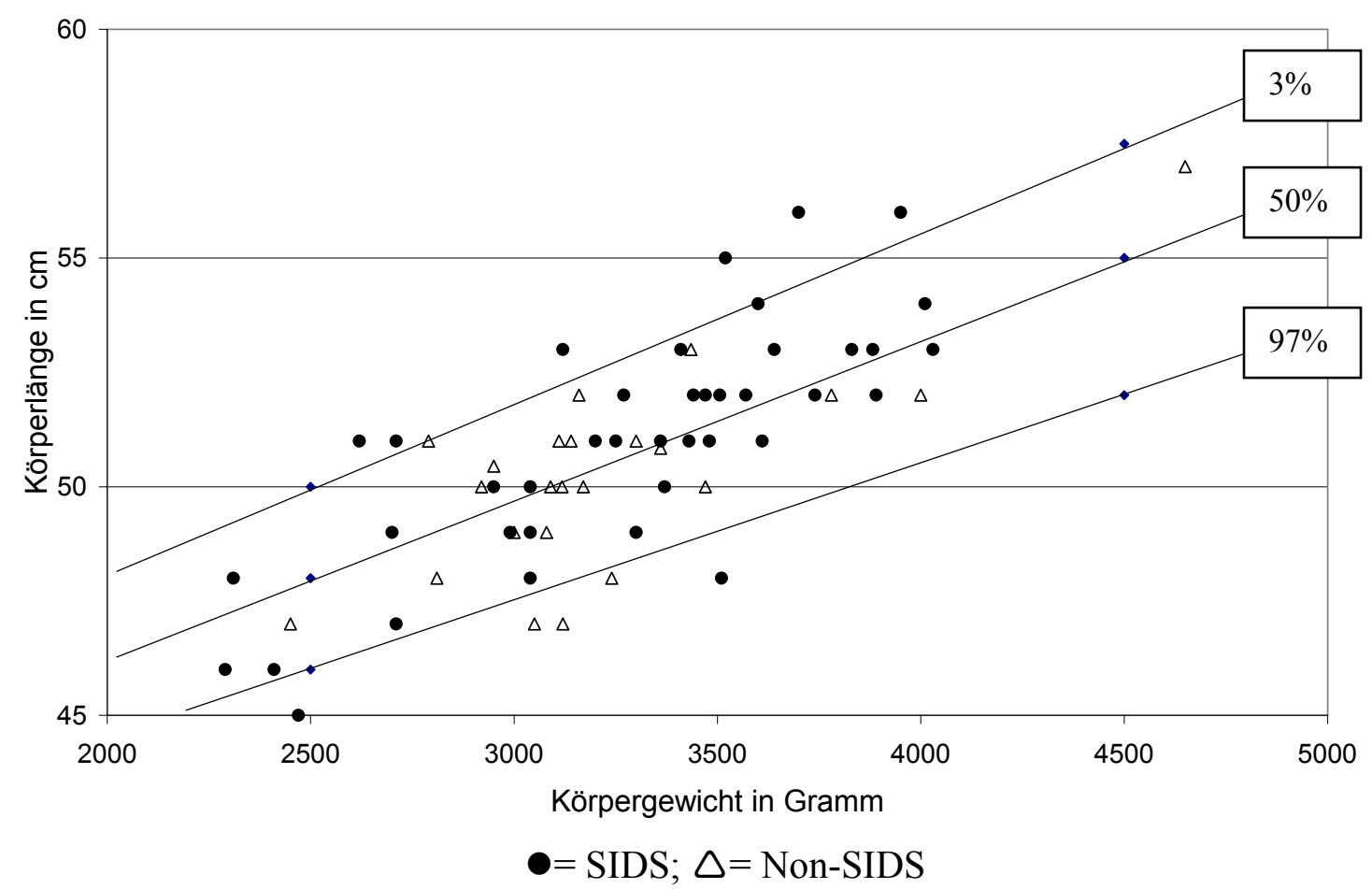

Zum Zeitpunkt der Obduktion zeigt sich eine generelle Verschiebung des Körpergewichts in Richtung der 3\%-Perzentile, die Kinder waren also im Allgemeinen zu leicht. Wir konnten in diese Auswertung 46 SIDS-Fälle und 23 Non-SIDS-Fälle aufnehmen. Genauer betrachtet zeigt sich, dass zwölf der SIDS-Fälle (26\%) und sechs der Non-SIDS-Fälle (ebenfalls 26\%) zu leicht waren. Zu schwer war ein SIDS-Kind (2\%) und ein Non-SIDS-Kind (4\%) (Abb. 21). 
Abb. 21: Obduktionsfälle BMBF-Studie, Institut f. Rechtsmedizin München 1999-2001: Perzentilen zum Zeitpunkt der Obduktion bei SIDS- und Non-SIDS-Fällen

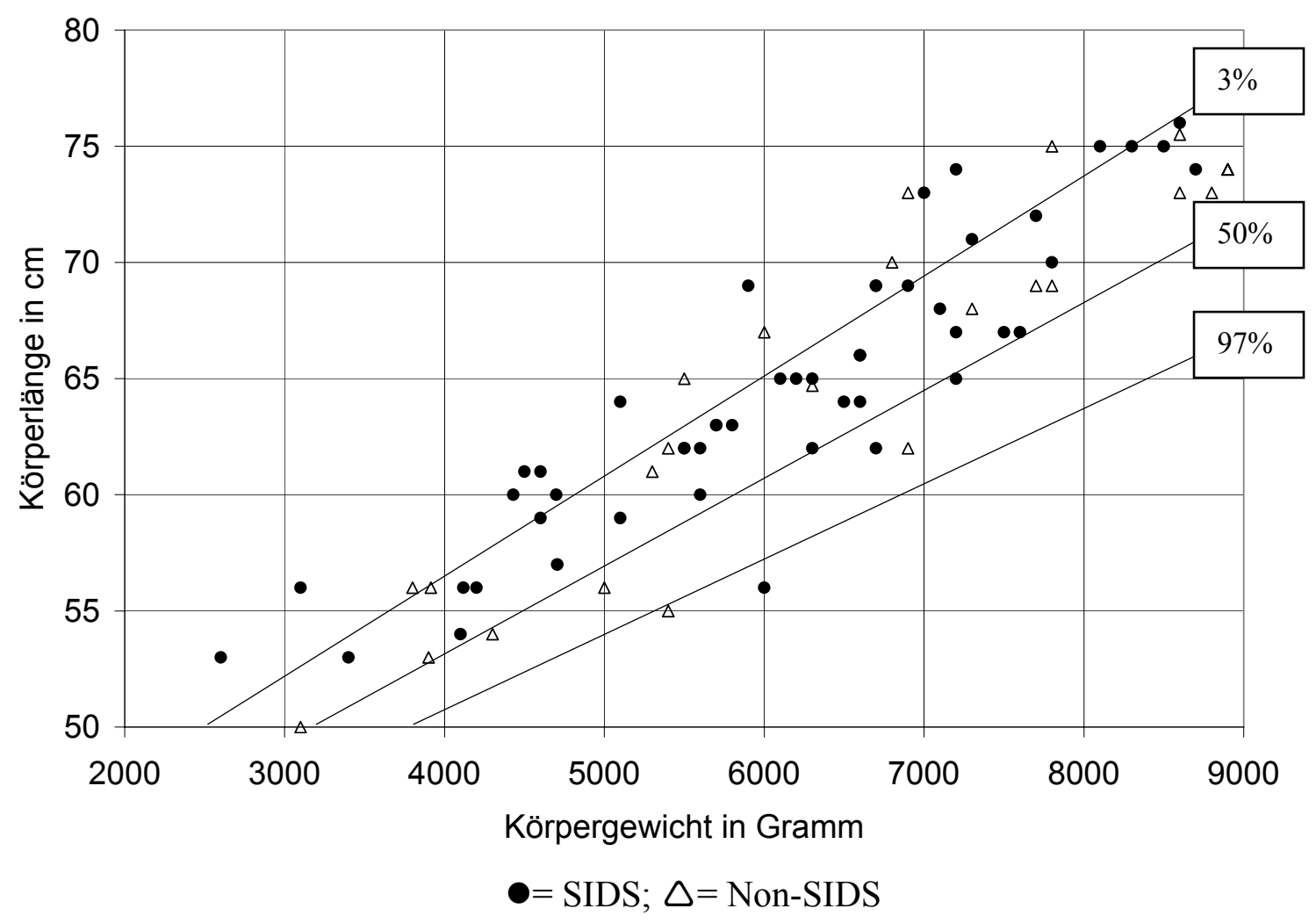

\subsubsection{Gesundheitszustand vor Todeseintritt}

Von den SIDS-Fällen verstarben 39\% aus akut anamnestisch erkranktem Zustand, bei den Non-SIDS-Fällen waren es 48\%. Aus anamnestisch gesundem Zustand heraus verstarben 51\% der SIDS-Fälle und 52\% der Non-SIDS-Fälle. Unter den SIDS-Fällen gab es fünf Kinder (10\%), die wenige Tage vor Todeseintritt krank waren, zum Todeszeitpunkt jedoch eigentlich wieder gesund. Solche Fälle fanden wir unter den Non-SIDS-Kindern nicht (Tabelle 44). 
Tabelle 44: Obduktionsfälle BMBF-Studie, Institut f. Rechtsmedizin München 19992001: Gesundheitszustand vor Todeseintritt bei SIDS- und Non-SIDS-Fällen (anamnestisch)

\begin{tabular}{|c|c|c|c|}
\cline { 2 - 4 } \multicolumn{1}{c|}{} & $\begin{array}{c}\text { krank unmittelbar } \\
\text { vor Todeseintritt }\end{array}$ & $\begin{array}{c}\text { krank in der letzten } \\
\text { Woche vor } \\
\text { Todeseintritt }\end{array}$ & $\begin{array}{c}\text { gesund seit } \\
\text { mindestens einer } \\
\text { Woche }\end{array}$ \\
\hline $\begin{array}{c}\text { SIDS } \\
(n=49=100 \%)\end{array}$ & 19 & 5 & 25 \\
\hline Non-SIDS & $(39 \%)$ & - & $(51 \%)$ \\
$(n=25=100 \%)$ & 12 & $(52 \%)$ \\
\hline
\end{tabular}

Untersucht man den Gesundheitszustand getrennt für Jungen und Mädchen, so zeigt sich bei den SIDS-Fällen, dass etwa doppelt so viele Jungen (50\%) wie Mädchen (26\%) aus akut erkranktem Zustand heraus verstarben (Tabelle 45).

Tabelle 45: Obduktionsfälle BMBF-Studie, Institut f. Rechtsmedizin München 19992001: Gesundheitszustand vor Todeseintritt bei den SIDS-Fällen

\begin{tabular}{|c|c|c|c|}
\cline { 2 - 4 } \multicolumn{1}{c|}{} & $\begin{array}{c}\text { krank unmittelbar } \\
\text { vor Todeseintritt }\end{array}$ & $\begin{array}{c}\text { krank in der letzten } \\
\text { Woche vor } \\
\text { Todeseintritt }\end{array}$ & $\begin{array}{c}\text { gesund seit } \\
\text { mindestens einer } \\
\text { Woche }\end{array}$ \\
\hline $\begin{array}{c}\text { weiblich } \\
(n=23=100 \%)\end{array}$ & 6 & 4 & 13 \\
\hline männlich & $(26 \%)$ & $17 \%)$ & $(57 \%)$ \\
$(n=26=100 \%)$ & 13 & $(4 \%)$ & 12 \\
\hline
\end{tabular}

Bei den Non-SIDS-Fällen zeigte sich kein solcher Unterschied zwischen den Geschlechtern (Tabelle 46).

Tabelle 46: Obduktionsfälle BMBF-Studie, Institut f. Rechtsmedizin München 19992001: Gesundheitszustand vor Todeseintritt bei den Non-SIDS-Fällen

\begin{tabular}{|c|c|c|c|}
\cline { 2 - 4 } & $\begin{array}{c}\text { krank unmittelbar } \\
\text { vor Todeseintritt }\end{array}$ & $\begin{array}{c}\text { krank in der letzten } \\
\text { Woche vor } \\
\text { Todeseintritt }\end{array}$ & $\begin{array}{c}\text { gesund seit } \\
\text { mindestens einer } \\
\text { Woche }\end{array}$ \\
\hline $\begin{array}{c}\text { weiblich } \\
(n=10=100 \%)\end{array}$ & 5 & - & 5 \\
männlich & $(50 \%)$ & - & $(50 \%)$ \\
\hline$n=15=100 \%)$ & 7 & $(53 \%)$ \\
\hline
\end{tabular}


Die Untersuchung, ob Kinder eines bestimmten Lebensalters gehäuft aus einem bestimmten Gesundheitszustand heraus verstarben, zeigte in der SIDS-Gruppe die meisten Todesfälle in akut krankem Zustand bei Kindern im vierten und fünften Lebensmonat (drei bzw. vier Todesfälle). Von den gesunden Kindern verstarben die meisten im dritten und vierten Lebensmonat (fünf bzw. vier Todesfälle) (Tabelle 47).

Tabelle 47: Obduktionsfälle BMBF-Studie, Institut f. Rechtsmedizin München 19992001: Gesundheitszustand vor Todeseintritt bezogen auf das Alter der SIDS-Kinder

\begin{tabular}{|c|c|c|c|c|c|c|c|c|c|c|c|c|}
\cline { 2 - 12 } \multicolumn{1}{c|}{} & \multicolumn{10}{c|}{ Alter der Kinder in Lebensmonaten } \\
\cline { 2 - 13 } \multicolumn{1}{c|}{} & 1 & 2 & 3 & 4 & 5 & 6 & 7 & 8 & 9 & 10 & 11 & 12 \\
\hline $\begin{array}{c}\text { krank unmittelbar } \\
\text { vor Todeseintritt } \\
(n=19=100 \%)\end{array}$ & 2 & - & - & 3 & 4 & 1 & 2 & 1 & 2 & 3 & 1 & - \\
\hline $\begin{array}{c}\text { krank in der } \\
\text { letzten Woche vor } \\
\text { Todeseintritt } \\
(n=5=100 \%)\end{array}$ & - & - & 2 & - & 1 & - & - & - & 2 & - & - & - \\
\hline $\begin{array}{c}\text { gesund seit } \\
\text { mindestens einer } \\
\begin{array}{c}\text { Woche } \\
(n=25=100 \%)\end{array}\end{array}$ & - & 3 & 5 & 4 & 3 & 1 & 3 & 1 & 3 & 1 & 1 & - \\
\hline
\end{tabular}

Bei den Non-SIDS-Fällen war die Beurteilung aufgrund der geringen Fallzahl schwierig. Bei den akut krank verstorbenen Kindern findet man keine Häufung in einem bestimmten Lebensalter. Es scheint jedoch, als wären besonders Kinder in den ersten vier Lebensmonaten aus gesundem Zustand heraus verstorben (Tabelle 48). 
Tabelle 48: Obduktionsfälle BMBF-Studie, Institut f. Rechtsmedizin München 19992001: Gesundheitszustand vor Todeseintritt bezogen auf das Alter der Non-SIDSKinder

\begin{tabular}{|c|c|c|c|c|c|c|c|c|c|c|c|c|}
\cline { 2 - 11 } \multicolumn{1}{c|}{} & \multicolumn{10}{c|}{ Alter der Kinder in Lebensmonaten } \\
\cline { 2 - 12 } \multicolumn{1}{c|}{} & 1 & 2 & 3 & 4 & 5 & 6 & 7 & 8 & 9 & 10 & 11 & 12 \\
\hline $\begin{array}{c}\text { krank unmittelbar } \\
\text { vor Todeseintritt } \\
(n=12=100 \%)\end{array}$ & 1 & 1 & 1 & 2 & 1 & - & - & 1 & - & 2 & 2 & 1 \\
\hline $\begin{array}{c}\text { krank in der } \\
\text { letzten Woche vor } \\
\text { Todeseintritt } \\
(n=0)\end{array}$ & - & - & - & - & - & - & - & - & - & - & - & - \\
\hline $\begin{array}{c}\text { gesund seit } \\
\text { mindestens einer } \\
\begin{array}{c}\text { Woche } \\
(n=13=100 \%)\end{array}\end{array}$ & 2 & 3 & 1 & 3 & - & 1 & 1 & - & 1 & 1 & - & - \\
\hline
\end{tabular}

Schließlich ließ sich feststellen, dass von den SIDS-Fällen, die aus akut krankem Zustand heraus verstarben, die meisten (37\%) in den Wintermonaten verstarben. Unter den aus gesundem Zustand heraus verstorbenen Kindern zeigte sich keine solche Bevorzugung einer Jahreszeit (Tabelle 49).

Tabelle 49: Obduktionsfälle BMBF-Studie, Institut f. Rechtsmedizin München 19992001: Gesundheitszustand vor Todeseintritt bezogen auf die Jahreszeit des Ablebens bei den SIDS-Fällen

\begin{tabular}{|c|c|c|c|c|}
\cline { 2 - 5 } \multicolumn{1}{c|}{} & $\begin{array}{c}\text { Frühling } \\
\text { (Mär, Apr, Mai) }\end{array}$ & $\begin{array}{c}\text { Sommer } \\
\text { (Jun, Jul, Aug) }\end{array}$ & $\begin{array}{c}\text { Herbst } \\
\text { (Sep, Okt, Nov) }\end{array}$ & $\begin{array}{c}\text { Winter } \\
\text { (Dez, Jan, Feb) }\end{array}$ \\
\hline $\begin{array}{c}\text { krank } \\
\text { unmittelbar vor } \\
\text { Todeseintritt } \\
(n=19=100 \%)\end{array}$ & 5 & 4 & 3 & 7 \\
\hline $\begin{array}{c}\text { krank in der } \\
\text { letzten Woche }\end{array}$ & 1 & $(21 \%)$ & $(16 \%)$ & $(37 \%)$ \\
$\begin{array}{c}\text { v. Todeseintritt } \\
(n=5=100 \%)\end{array}$ & $(20 \%)$ & $(40 \%)$ & $(20 \%)$ & $(20 \%)$ \\
\hline $\begin{array}{c}\text { gesund seit } \\
\text { mindestens } \\
\text { einer Woche } \\
(n=25=100 \%)\end{array}$ & $(24 \%)$ & $(28 \%)$ & $\begin{array}{c}7 \\
(28 \%)\end{array}$ & $\begin{array}{c}5 \\
(20 \%)\end{array}$ \\
\hline
\end{tabular}


Bei den Kindern aus der Non-SIDS-Gruppe verstarben aus akut krankem Zustand die Hälfte (50\%) in den Wintermonaten. Bei Kindern, die in gesundem Zustand verstarben, waren die Herbst- und Wintermonate die am häufigsten betroffene Jahreszeit (insgesamt 70\%, Tabelle 50).

Tabelle 50: Obduktionsfälle BMBF-Studie, Institut f. Rechtsmedizin München 19992001: Gesundheitszustand vor Todeseintritt bezogen auf die Jahreszeit des Ablebens bei den Non-SIDS-Fällen

\begin{tabular}{|c|c|c|c|c|}
\cline { 2 - 5 } \multicolumn{1}{c|}{} & $\begin{array}{c}\text { Frühling } \\
\text { (Mär, Apr, Mai) }\end{array}$ & $\begin{array}{c}\text { Sommer } \\
\text { (Jun, Jul, Aug) }\end{array}$ & $\begin{array}{c}\text { Herbst } \\
\text { (Sep, Okt, Nov) }\end{array}$ & $\begin{array}{c}\text { Winter } \\
\text { (Dez, Jan, Feb) }\end{array}$ \\
\hline $\begin{array}{c}\text { krank } \\
\text { unmittelbar vor } \\
\text { Todeseintritt } \\
(n=12=100 \%)\end{array}$ & 3 & 2 & 1 & 6 \\
\hline $\begin{array}{c}\text { krank in der } \\
\text { letzten Woche } \\
\text { vor Todes- } \\
\text { eintritt }(n=0)\end{array}$ & - & $(17 \%)$ & $(8 \%)$ & $(50 \%)$ \\
\hline $\begin{array}{c}\text { gesund seit } \\
\text { mindestens } \\
\text { einer Woche } \\
(n=13=100 \%)\end{array}$ & $(15 \%)$ & - & - & - \\
\hline
\end{tabular}

\subsubsection{Geschwister}

Von den verstorbenen SIDS-Fällen waren 20\% Einzelkinder, bzw. Erstgeborene. Unter den Non-SIDS-Fällen waren dies mehr als doppelt so viele, nämlich $42 \%$.

Die SIDS-Fällen betrafen insgesamt am häufigsten Kinder mit einem Geschwisterkind (54\%), dann nahm die Anzahl der Todesfälle mit der Anzahl der Geschwister ab.

Unter den Non-SIDS-Fällen waren am häufigsten (42\%) Einzelkinder/ Erstgeborene betroffen, es folgten Kinder mit einem (32\%), zwei (21\%) und schließlich mit drei (5\%) Geschwistern (Tabelle 51). 
Tabelle 51: Obduktionsfälle BMBF-Studie, Institut f. Rechtsmedizin München 19992001: Anzahl der Geschwisterkinder von SIDS- und Non-SIDS-Fällen

\begin{tabular}{|c|c|c|c|c|c|}
\cline { 2 - 6 } \multicolumn{1}{c|}{} & 0 & 1 & 2 & 3 & 4 und mehr \\
\hline $\begin{array}{c}\text { SIDS } \\
(n=46= \\
100 \%)\end{array}$ & $\begin{array}{c}9 \\
(20 \%)\end{array}$ & $\begin{array}{c}25 \\
(54 \%)\end{array}$ & $\begin{array}{c}8 \\
(17 \%)\end{array}$ & $\begin{array}{c}3 \\
(7 \%)\end{array}$ & $\begin{array}{c}1 \\
(2 \%)\end{array}$ \\
\hline $\begin{array}{c}\text { Non-SIDS } \\
(n=19= \\
100 \%)\end{array}$ & $\begin{array}{c}8 \\
(42 \%)\end{array}$ & $\begin{array}{c}6 \\
(32 \%)\end{array}$ & $\begin{array}{c}4 \\
(21 \%)\end{array}$ & $\begin{array}{c}1 \\
(5 \%)\end{array}$ & - \\
\hline
\end{tabular}

\subsubsection{Alter der Eltern bei der Geburt des verstorbenen Kindes}

Das Durchschnittsalter der SIDS-Mütter lag bei 26,5 Jahren, das der Non-SIDS-Mütter bei 26,1 Jahren und das durchschnittliche Alter einer Mutter bei der Geburt des Kindes laut Statistischem Bundesamt bei 29,3 Jahren [Statistisches Bundesamt 2000]. Die Väter der SIDS-Kinder waren im Durchschnitt 30,8 Jahre alt, die der Non-SIDS-Fälle 29,6 Jahre. Das Alter der Mutter bei der Geburt des verstorbenen Kindes ist im folgenden Diagramm dargestellt. Der Verlauf der Alterskurven der SIDS- und NonSIDS-Mütter verhält sich prinzipiell gleichsinnig, nur die prozentualen Anteile der jeweiligen Altersgruppe unterscheiden sich etwas (Abb. 22). 
Abb. 22: Obduktionsfälle BMBF-Studie, Institut f. Rechtsmedizin München 1999-2001: Alter der Mutter bei der Geburt des SIDS- bzw. Non-SIDS-Kindes

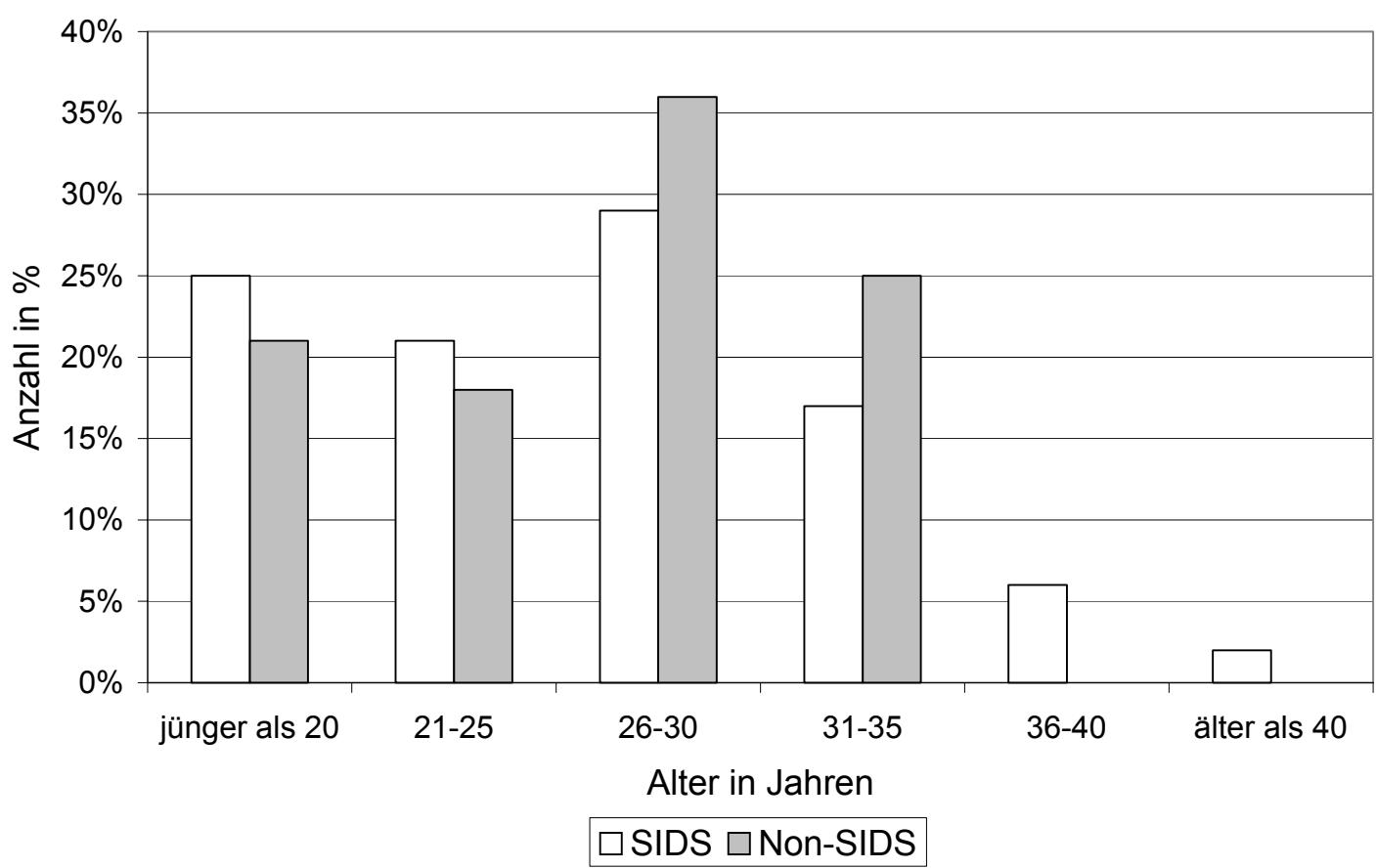

Betrachtet man das Alter der Väter so zeigt sich auch hier ein ähnliches Altersprofil sowohl bei der SIDS- als auch bei der Non-SIDS-Gruppe (Abb. 23). 
Abb. 23: Obduktionsfälle BMBF-Studie, Institut f. Rechtsmedizin München 1999-2001: Alter des Vaters bei der Geburt des SIDS- bzw. Non-SIDS-Kindes

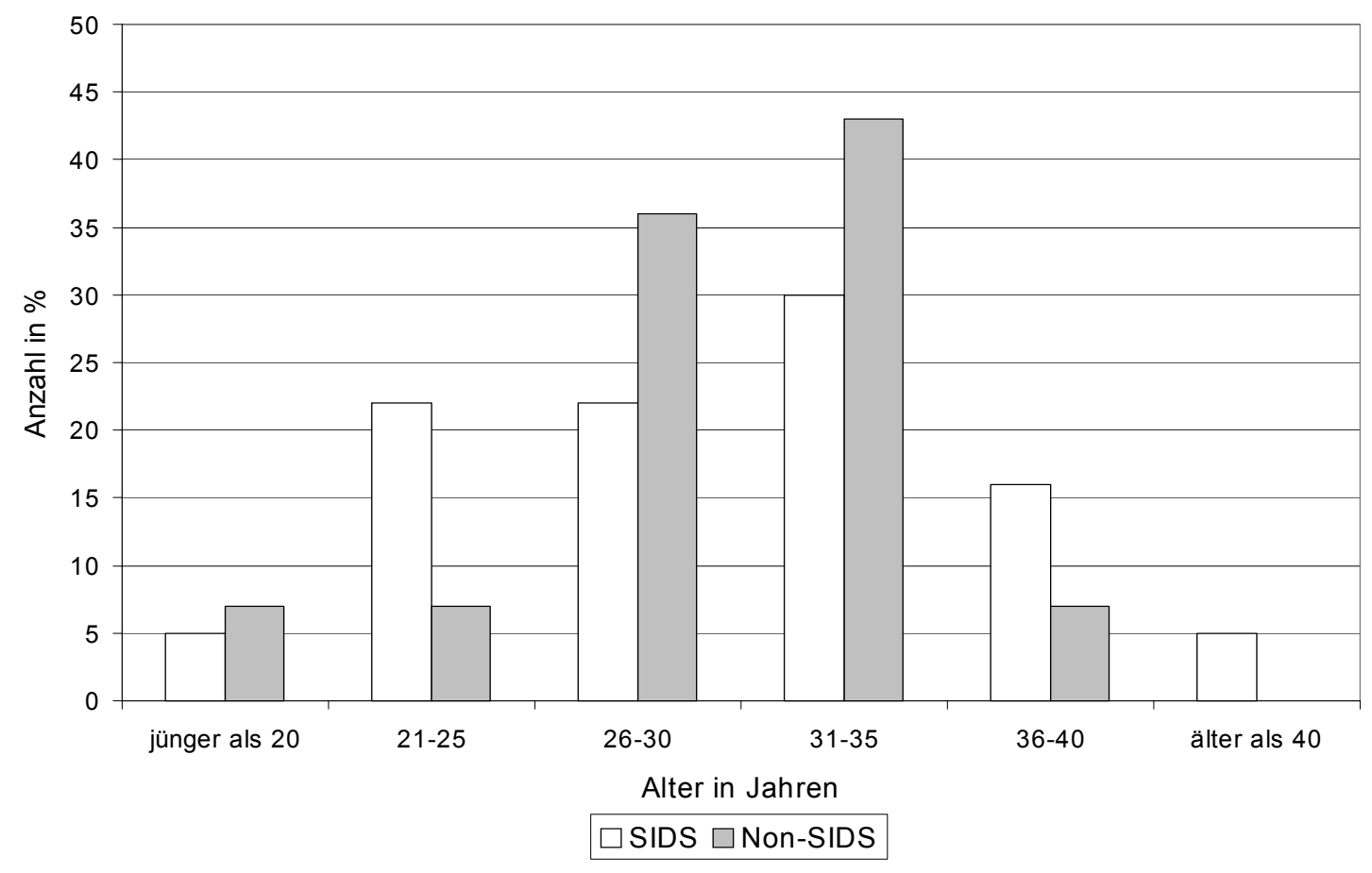

\subsubsection{Soziale Stellung der Familie}

Wie schon in Kapitel 3.1.12. wurde das jeweils höchste Ausbildungsniveau der Eltern der Gruppe 1 bis 4 zugeordnet, wobei die Gruppe 1, wie schon in Kapitel 3.1.12. erläutert, in die Beurteilung nur eingeschränkt einbezogen werden kann:

Gruppe 1: Hausfrauen/ -männer, Arbeitslose, Nichterwerbstätige

Gruppe 2: Hilfsarbeiter, Tätigkeiten ohne erfolgte Berufsausbildung

Gruppe 3: abgeschlossene Berufsausbildung

Gruppe 4: abgeschlossenes Studium an der Universität oder Fachhochschule

In der SIDS-Gruppe waren bei einem Viertel (25\%) der Kinder der bestausgebildete oder beide Elternteile der Gruppe 1 zuzuordnen. In der Non-SIDS-Gruppe war dies nur bei 18,7\% der Fall. Der Großteil (43,2\%) der Familien der SIDS-Fälle wurde jedoch der Ausbildungsgruppe 3 zugeordnet, in der Non-SIDS-Gruppe war am häufigsten (37,5\%) das Ausbildungsniveau 2 zu finden (Tabelle 52). 
Tabelle 52: Obduktionsfälle BMBF-Studie, Institut f. Rechtsmedizin München 19992001: Höchstes Ausbildungsniveau der Eltern

\begin{tabular}{|c|c|c|c|c|}
\cline { 2 - 5 } \multicolumn{1}{c|}{} & 1 & 2 & 3 & 4 \\
\hline SIDS & 11 & 10 & 19 & 4 \\
$(n=44=100 \%)$ & $(25 \%)$ & $(23 \%)$ & $(43 \%)$ & $(9 \%)$ \\
\hline Non-SIDS & 3 & 6 & 5 & 2 \\
$(n=16=100 \%)$ & $(19 \%)$ & $(37 \%)$ & $(31 \%)$ & $(13 \%)$ \\
\hline
\end{tabular}

Von den vier getöteten Kindern erhielten wir in zwei Fällen keine Angaben zum Ausbildungsniveau der Eltern, in einem Fall gehörten beide Eltern der Gruppe 1 an, im anderen Fall war die Mutter der Gruppe 1, der Vater der Gruppe 2 zuzuordnen.

\subsubsection{2. „Postvitales“ Intervall}

Die Zeitspanne zwischen dem Zeitpunkt, zu dem das Kind letztmalig lebend gesehen wurde, und der Totauffindung lag bei den SIDS-Fällen zu 6\% unter einer Stunde. Der Großteil der Kinder (60\%) wurde innerhalb von maximal fünf Stunden nach möglichem Todeseintritt aufgefunden.

In der Non-SIDS-Gruppe wurden 13\% der Kinder innerhalb der ersten Stunde und 50\% innerhalb der ersten fünf Stunden nach möglichem Todeseintritt aufgefunden.

Bei den SIDS-Fällen wurde bei $9 \%$ der Kinder der Tod erst nach frühestens zehn Stunden entdeckt, in der Gruppe der Non-SIDS-Fälle war dies bei 6\% der Fall (Tabelle 53).

Tabelle 53: Obduktionsfälle BMBF-Studie, Institut f. Rechtsmedizin München 19992001: „Postvitales“ Intervall bei SIDS- und Non-SIDS-Fällen

\begin{tabular}{|c|c|c|c|c|}
\cline { 2 - 5 } \multicolumn{1}{c|}{} & weniger als $1 \mathrm{~h}$ & $1 \mathrm{bis} 5 \mathrm{~h}$ & 6 bis $10 \mathrm{~h}$ & mehr als $10 \mathrm{~h}$ \\
\hline SIDS & 3 & 28 & 12 & 4 \\
$(n=47=100 \%)$ & $(6 \%)$ & $(60 \%)$ & $(26 \%)$ & $(8 \%)$ \\
\hline Non-SIDS & 2 & 8 & 5 & 1 \\
$(n=16=100 \%)$ & $(13 \%)$ & $(50 \%)$ & $(31 \%)$ & $(6 \%)$ \\
\hline
\end{tabular}


Betrachtet man das postvitale Intervall für Jungen und Mädchen getrennt voneinander, so zeigt sich in der Gruppe der SIDS-Fälle, dass insgesamt 45\% der Mädchen innerhalb von fünf Stunden aufgefunden wurden, jedoch $82 \%$ der verstorbenen Jungen (Tabelle $54)$.

Tabelle 54: Obduktionsfälle BMBF-Studie, Institut f. Rechtsmedizin München 19992001: „Postvitales “ Intervall bei SIDS-Fällen, geschlechtsbezogen

\begin{tabular}{|c|c|c|c|c|}
\cline { 2 - 5 } \multicolumn{1}{c|}{} & weniger als $1 \mathrm{~h}$ & $1-5 \mathrm{~h}$ & $6-10 \mathrm{~h}$ & mehr als $10 \mathrm{~h}$ \\
\hline weiblich & 2 & 7 & 9 & 2 \\
$(n=20=100 \%)$ & $(10 \%)$ & $(35 \%)$ & $(45 \%)$ & $(10 \%)$ \\
\hline männlich & - & 22 & 3 & 2 \\
$(n=27=100 \%)$ & - & $(82 \%)$ & $(11 \%)$ & $(7 \%)$ \\
\hline
\end{tabular}

Bei den Kinder der Non-SIDS-Gruppe wurden 57\% der Mädchen und insgesamt 67\% der Jungen innerhalb der ersten fünf Stunden nach möglichem Todeseintritt tot aufgefunden (Tabelle 55).

Tabelle 55: Obduktionsfälle BMBF-Studie, Institut f. Rechtsmedizin München 19992001: „Postvitales“ Intervall bei Non-SIDS-Fällen, geschlechtsbezogen

\begin{tabular}{|c|c|c|c|c|}
\cline { 2 - 5 } \multicolumn{1}{c|}{} & weniger als 1h & $1-5 \mathrm{~h}$ & $6-10 \mathrm{~h}$ & mehr als $10 \mathrm{~h}$ \\
\hline $\begin{array}{c}\text { weiblich } \\
(n=7=100 \%)\end{array}$ & - & 4 & 2 & 1 \\
$(57 \%)$ & $(29 \%)$ & $(14 \%)$ \\
\hline männlich & 2 & 4 & 3 & - \\
$(n=9=100 \%)$ & $(22 \%)$ & $(44 \%)$ & $(34 \%)$ & - \\
\hline
\end{tabular}

\subsubsection{Reanimation durch einen Notarzt}

Es wurde die Hälfte aller SIDS-Fälle (50\%) reanimiert. Bei den Non-SIDS-Fällen wurde in zwei Drittel der Fälle (67\%) eine Reanimation durchgeführt (Tabelle 56). 
Tabelle 56: Obduktionsfälle BMBF-Studie, Institut f. Rechtsmedizin München 19992001: Durchgeführte Reanimationen bei SIDS- und Non-SIDS-Fällen

\begin{tabular}{|c|c|c|}
\cline { 2 - 3 } \multicolumn{1}{c|}{} & reanimiert & nicht reanimiert \\
\hline SIDS & 18 & 18 \\
$(n=36=100 \%)$ & $(50 \%)$ & $(50 \%)$ \\
\hline Non-SIDS & 12 & 6 \\
$(n=18=100 \%)$ & $(67 \%)$ & $(33 \%)$ \\
\hline
\end{tabular}

Nimmt man nun wieder eine getrennte Betrachtung von Jungen und Mädchen vor, so zeigt sich in der Gruppe der SIDS-Fälle, dass 47\% der Mädchen und 53\% der Jungen reanimiert wurden (Tabelle 57).

Tabelle 57: Obduktionsfälle BMBF-Studie, Institut f. Rechtsmedizin München 19992001: Reanimationen bei SIDS-Fällen, geschlechtsbezogen

\begin{tabular}{|c|c|c|}
\hline & reanimiert & nicht reanimiert \\
\hline $\begin{array}{c}\text { weiblich } \\
(n=1=100 \% 7)\end{array}$ & $\begin{array}{c}8 \\
(47 \%)\end{array}$ & $\begin{array}{c}9 \\
(53 \%)\end{array}$ \\
\hline $\begin{array}{c}\text { männlich } \\
(n=19=100 \%)\end{array}$ & $\begin{array}{c}10 \\
(53 \%)\end{array}$ & $\begin{array}{c}9 \\
(47 \%)\end{array}$ \\
\hline
\end{tabular}

Bei den Non-SIDS-Fällen hingegen wurden etwa doppelt so viele Jungen (82\%) reanimiert wie Mädchen (43\%)(Tabelle 58).

Tabelle 58: Obduktionsfälle BMBF-Studie, Institut f. Rechtsmedizin München 19992001: Reanimationen bei Non-SIDS-Fällen, geschlechtsbezogen

\begin{tabular}{|c|c|c|}
\hline & reanimiert & nicht reanimiert \\
\hline $\begin{array}{c}\text { weiblich } \\
(n=7=100 \%)\end{array}$ & $\begin{array}{c}3 \\
(43 \%)\end{array}$ & $\begin{array}{c}4 \\
(57 \%)\end{array}$ \\
\hline $\begin{array}{c}\text { männlich } \\
(n=11=100 \%)\end{array}$ & $\begin{array}{c}9 \\
(82 \%)\end{array}$ & $\begin{array}{c}2 \\
(18 \%)\end{array}$ \\
\hline
\end{tabular}




\subsection{Obduktionsbefunde}

Unterteilt man die in Kapitel 3.2. dargestellten Obduktionsbefunde in die zwei Kategorien "SIDS" und "Non-SIDS" auf, so ergeben sich die in der folgenden Tabelle dargestellten Häufigkeiten der einzelnen pathologischen Veränderungen.

Petechien an Thymus, Epikard, Perikard und Pleura kamen gehäuft in der SIDS-Gruppe vor, Petechien an den Lid- und Bindehäuten häufiger in der Non-SIDS-Gruppe. Bei Untersuchung der Anzahl der Thymus-Petechien fanden sich in den einzelnen Kategorien folgende Befunde (Tabelle 59):

Tabelle 59: Obduktionsfälle BMBF-Studie, Institut f. Rechtsmedizin München 19992001: Anzahl der Thymus-Petechien bezogen auf die Kategorie

\begin{tabular}{|c|c|c|c|c|}
\cline { 2 - 5 } \multicolumn{1}{c|}{} & \multicolumn{4}{c|}{ Anzahl der Thymus-Petechien } \\
\hline Kategorie & keine & wenig & mittel & massenhaft \\
\hline $\begin{array}{c}1 \\
(\mathrm{n}=5=100 \%)\end{array}$ & - & $1(20 \%)$ & $4(80 \%)$ & - \\
\hline $\begin{array}{c}2 \\
(\mathrm{n}=30=100 \%)\end{array}$ & $5(17 \%)$ & $3(10 \%)$ & $18(60 \%)$ & $4(13 \%)$ \\
\hline $\begin{array}{c}3 \\
(\mathrm{n}=11=100 \%)\end{array}$ & - & $2(18 \%)$ & $7(64 \%)$ & $2(18 \%)$ \\
\hline $\begin{array}{c}4 \\
(\mathrm{n}=8=100 \%)\end{array}$ & $2(25 \%)$ & $2(25 \%)$ & $2(25 \%)$ & $2(25 \%)$ \\
\hline
\end{tabular}

Weiter zeigten die SIDS-Fälle häufiger vergrößerte Lymphknoten, gerötete Luftwege, „gummiartige Beschaffenheit“ der Lungen (entspricht Dystelektasen), Mittelohrentzündungen, eine aufgelockert erscheinende Milz, Lungenödeme, Einblutungen in die Halsmuskulatur, Fehlbildungen und eingerollte Epiglottiden. Bei den Non-SIDS-Fällen hingegen fanden sich häufiger Anzeichen für Hirndruck, agonales Erbrechen eventuell mit Aspiration, pathologische kardiale Befunde, Perikardergüsse, Anzeichen für Mißhandlungen und in einem Fall eine nekrotisierende Enteritis (Tabelle $60)$. 
Tabelle 60: Obduktionsfälle BMBF-Studie, Institut f. Rechtsmedizin München 19992001: Obduktionsbefunde bei SIDS- und Non-SIDS-Fällen

\begin{tabular}{|l|l|l|}
\hline \multicolumn{1}{|c|}{ Befund } & \multicolumn{1}{c|}{$\begin{array}{c}\text { SIDS } \\
(n=51=100 \%)\end{array}$} & \multicolumn{1}{|c|}{$\begin{array}{c}\text { Non-SIDS } \\
(n=28=100 \%)\end{array}$} \\
\hline Petechien: Thymus & $43(84 \%)$ & $17(61 \%)$ \\
\hline Petechien: am Herz(-beutel) & $31(61 \%)$ & $12(43 \%)$ \\
\hline Petechien: subpleural & $23(45 \%)$ & $8(29 \%)$ \\
\hline Petechien: Lid- und/ oder Bindehäute & $12(24 \%)$ & $12(43 \%)$ \\
\hline vergrößerte Hals-/ Gekröselymphknoten & $27(53 \%)$ & $12(43 \%)$ \\
\hline Hirndruck & $16(31 \%)$ & $15(54 \%)$ \\
\hline gerötete Luftwege & $21(41 \%)$ & $8(29 \%)$ \\
\hline gummiartige Beschaffenheit der Lungen & $16(31 \%)$ & $6(21 \%)$ \\
\hline Mittelohrentzündung eitrig/ serös & $15(29 \%)$ & $5(18 \%)$ \\
\hline aufgelockerte Milz & $13(26 \%)$ & $4(14 \%)$ \\
\hline Lungenödem & $12(24 \%)$ & $5(18 \%)$ \\
\hline agonales Erbrechen / Aspiration & $6(12 \%)$ & $4(14 \%)$ \\
\hline pathologische kardiale Befunde & $2(4 \%)$ & $5(18 \%)$ \\
\hline Einblutungen in die Halsmuskulatur & $5(10 \%)$ & $1(4 \%)$ \\
\hline Perikarderguß & $2(4 \%)$ & $4(14 \%)$ \\
\hline Fehlbildungen & $2(4 \%)$ & $3(4 \%)$ \\
\hline Anzeichen für Mißhandlungen & - & $4(14 \%)$ \\
\hline eingerollte Epiglottis & $3(6 \%)$ & $1(4 \%)$ \\
\hline nekrotisierende Enteritis & - & $1(4 \%)$ \\
\hline
\end{tabular}

\subsection{Histologie}

In der histologischen Untersuchung zeigte sich, dass unter den SIDS-Fällen lediglich Lungenveränderungen in Form von stattgehabter Aspiration und in einem Fall eine Nierentubulusnekrose häufiger vorkamen, als in der Non-SIDS-Gruppe.

Sämtliche sonstigen histologischen Befunde waren prozentual betrachtet in der NonSIDS-Gruppe häufiger vorzufinden als in der Gruppe der SIDS-Fälle (Tabelle 61). 
Tabelle 61: Obduktionsfälle BMBF-Studie, Institut f. Rechtsmedizin München 19992001: Histologische Befunde bei SIDS- und Non-SIDS-Fällen

\begin{tabular}{|l|l|l|}
\hline \multicolumn{1}{|c|}{ Befunde } & \multicolumn{1}{c|}{$\begin{array}{c}\text { SIDS } \\
(n=51=100 \%)\end{array}$} & \multicolumn{1}{|c|}{$\begin{array}{c}\text { Non-SIDS } \\
(n=28=100 \%)\end{array}$} \\
\hline Laryngitis & $28(55 \%)$ & $16(57 \%)$ \\
\hline Tracheitis & $24(47 \%)$ & $16(57 \%)$ \\
\hline Enteritis & $16(31 \%)$ & $12(43 \%)$ \\
\hline Rhinitis/ Pharyngitis & $15(29 \%)$ & $10(36 \%)$ \\
\hline Pneumonie & $10(20 \%)$ & $11(39 \%)$ \\
\hline Bronchiolitis/ Bronchitis & $11(22 \%)$ & $9(32 \%)$ \\
\hline Kolitis & $10(20 \%)$ & $9(32 \%)$ \\
\hline Tonsillitis & $9(18 \%)$ & $8(29 \%)$ \\
\hline Sialadenitis & $8(16 \%)$ & $5(18 \%)$ \\
\hline Hepatitis & $3(6 \%)$ & $9(32 \%)$ \\
\hline Z.n. Aspiration & $5(10 \%)$ & $2(7 \%)$ \\
\hline Gastritis & $1(2 \%)$ & $2(7 \%)$ \\
\hline Epikarditis/ Myokarditis & - & $3(11 \%)$ \\
\hline Nephritis & $1(2 \%)$ & $1(4 \%)$ \\
\hline Pankreatitis & - & $2(7 \%)$ \\
\hline
\end{tabular}

Auch hier sei noch einmal erwähnt, dass es sich an sämtlichen Organen fast ausnahmslos um lymphozytäre Entzündungsreaktionen handelte.

\subsection{Bakteriologie}

Die durchgeführten bakteriologischen Untersuchungen wiesen bei sechzehn SIDSKindern (31\%) und bei neun (32\%) der Non-SIDS-Fälle eine bakterielle Infektion nach (Tabelle 62). 
Tabelle 62: Obduktionsfälle BMBF-Studie, Institut f. Rechtsmedizin München 19992001: Bakteriologischer Erregernachweis bei SIDS- und Non-SIDS-Fällen

\begin{tabular}{|c|c|c|c|c|c|}
\hline & $\begin{array}{c}\text { Staphylo- } \\
\text { kokkus } \\
\text { aureus }\end{array}$ & $\begin{array}{c}\text { Strepto- } \\
\text { kokkus } \\
\text { pneumoniae }\end{array}$ & $\begin{array}{l}\text { andere } \\
\text { Strepto- } \\
\text { kokken }\end{array}$ & $\begin{array}{c}\text { Klebsiella } \\
\text { pneumoniae }\end{array}$ & $\begin{array}{c}\text { Neisseria } \\
\text { meningitidis }\end{array}$ \\
\hline $\begin{array}{c}\text { SIDS } \\
(n=51= \\
100 \%)\end{array}$ & $\begin{array}{c}10 \\
(20 \%)\end{array}$ & $\begin{array}{c}1 \\
(2 \%)\end{array}$ & $\begin{array}{c}4 \\
(8 \%)\end{array}$ & $\begin{array}{c}2 \\
(4 \%)\end{array}$ & - \\
\hline $\begin{array}{c}\text { Non-SIDS } \\
(n=28= \\
100 \%))\end{array}$ & $\begin{array}{c}2 \\
(7 \%)\end{array}$ & $\begin{array}{c}1 \\
(4 \%)\end{array}$ & $\begin{array}{c}5 \\
(18 \%)\end{array}$ & - & $\begin{array}{c}3 \\
(11 \%)\end{array}$ \\
\hline
\end{tabular}

Unter den SIDS-Fällen gab es einen Fall einer Doppelinfektion mit Staphylokokkus aureus und Streptokokken. Bei den Non-SIDS-Fällen gab es eine Doppelinfektion mit Streptokokken und Neisseria meningitidis und eine Doppelinfektion mit Staphylokokkus aureus und Streptokokken:

\subsection{Virologie}

Von den SIDS-Fällen wiesen, unter Beachtung der Doppelinfektionen und unter Ausschluss der durch Zusatzuntersuchungen nachgewiesenen Influenza A- und HHV-6Infektion bei zwei Non-SIDS-Fällen, 18\% eine Virusinfektion auf, bei den Non-SIDSFällen waren es 39\% (Tabelle 63).

Tabelle 63: Obduktionsfälle BMBF-Studie, Institut f. Rechtsmedizin München 19992001: Virusnachweis bei SIDS- und Non-SIDS-Fällen

\begin{tabular}{|c|c|c|c|c|c|c|}
\hline & \multicolumn{6}{|c|}{ Virus } \\
\hline Kategorie & $\begin{array}{l}\text { Parain- } \\
\text { fluenza }\end{array}$ & RS-Viren & $\begin{array}{l}\text { Adeno- } \\
\text { viren }\end{array}$ & $\begin{array}{l}\text { Zytome- } \\
\text { galie-Viren }\end{array}$ & $\begin{array}{l}\text { Entero- } \\
\text { viren }\end{array}$ & Rotaviren \\
\hline $\begin{array}{c}\text { SIDS } \\
(\mathrm{n}=51 \\
=100 \%)\end{array}$ & - & - & 4 & 5 & 1 & - \\
\hline $\begin{array}{c}\text { Non- } \\
\text { SIDS(n=2 } \\
8 \\
=100 \%)\end{array}$ & 3 & 1 & - & 7 & - & - \\
\hline
\end{tabular}


Eine Doppelinfektion mit Adeno- und Enteroviren, allerdings "nur" in der Stuhlprobe, kam in der Gruppe der SIDS-Fälle vor, unter den Non-SIDS-Fällen faden sich zwei Kinder mit Doppelinfektionen (Parainfluenza- und Zytomegalieviren, RS- und Zytomegalieviren). Zwei der getöteten Kinder wiesen eine Zytomegalieinfektion auf.

\subsection{Toxikologie}

Die toxikologische Untersuchung erbrachte bei zwei SIDS-Fällen den Nachweis einer Spur Carbamazepin beziehungsweise einer Spur Morphin.

Bei den Non-SIDS-Fällen wies ein Kind eine Spur Lidocain, ein Kind Morphin $(0,002 \mathrm{mg} / \mathrm{l})$, ein Kind Paracetamol (7,5mg/l) und ein Kind Phenobarbital (44,9mg/l) plus Phenytoin $(14,45 \mathrm{mg} / \mathrm{l})$, Thiopental $(3,9 \mathrm{mg} / \mathrm{l})$ und Diazepam $(0,225 \mathrm{mg} / \mathrm{l})$ auf.

\subsection{Ethanolbestimmung und Begleitstoffanalyse}

\subsubsection{Ethanol}

Von den neun Kindern mit erhöhten Ethanolspiegeln (mehr als 0,1 Promille) waren acht der SIDS-Gruppe zuzuordnen, ein Kind den Non-SIDS-Fällen (Kategorie 4). Die SIDSFälle zeigten folgende Merkmale (Tabelle 64): 
Tabelle 64: Obduktionsfälle BMBF-Studie, Institutf. Rechtsmedizin München 19992001: SIDS-Fälle mit unphysiologisch hohen Serumethanolspiegeln

\begin{tabular}{|c|c|c|c|c|c|}
\hline $\begin{array}{c}\text { Ethanol- } \\
\text { spiegel (\%o) }\end{array}$ & Geschlecht & $\begin{array}{c}\text { Alter } \\
\text { (Monate) }\end{array}$ & $\begin{array}{c}\text { postmortales } \\
\text { Intervall }\end{array}$ & Reanimation & $\begin{array}{c}\text { Ausbildungs } \\
\text { niveau Eltern }\end{array}$ \\
\hline 0,35 & $\mathrm{~m}$ & 7 & 38 & nein & 3 \\
\hline 0,11 & $\mathrm{w}$ & 9 & 62 & ja & 2 \\
\hline 0,26 & $\mathrm{~m}$ & 2 & 26 & nein & 3 \\
\hline 0,13 & $\mathrm{w}$ & 3 & 13 & nein & 2 \\
\hline 0,31 & $\mathrm{~m}$ & 10 & 16 & nein & 1 \\
\hline 0,11 & $\mathrm{~m}$ & 4 & 29 & ja & 3 \\
\hline 0,30 & $\mathrm{~m}$ & 5 & 8 & nein & 1 \\
\hline 0,20 & $\mathrm{w}$ & 5 & 16 & nein & 2 \\
\hline
\end{tabular}

\subsubsection{Acetaldehyd}

Physiologische, also endogene Acetaldehydspiegel liegen bei bis zu 5,1 mg/1 [Wittmann 1996]. Bei den von uns untersuchten Fällen lagen die Acetaldehydspiegel in sechs Fällen bei mehr als 5,1mg/l. Es handelte sich dabei um fünf SIDS-Fälle und einen NonSIDS-Fall. Bei den SIDS-Fällen ergaben sich folgende Beobachtungen (Tabelle 65):

Tabelle 65: Obduktionsfälle BMBF-Studie, Institut f. Rechtsmedizin München 19992001: SIDS-Fälle mit unphysiologisch hohen Acetaldehydspiegeln

\begin{tabular}{|c|c|c|c|c|c|}
\hline $\begin{array}{c}\text { Acetaldehyd } \\
(\mathrm{mg} / \mathrm{l})\end{array}$ & Geschlecht & $\begin{array}{c}\text { Alter } \\
(\text { Monate })\end{array}$ & $\begin{array}{c}\text { postmortales } \\
\text { Intervall }\end{array}$ & Reanimation & $\begin{array}{c}\text { Ausbildungs- } \\
\text { niveau Eltern }\end{array}$ \\
\hline 5,28 & $\mathrm{~m}$ & 7 & $38 \mathrm{~h}$ & nein & 3 \\
\hline 5,94 & $\mathrm{~m}$ & 3 & $57 \mathrm{~h}$ & ja & - \\
\hline 5,91 & $\mathrm{~m}$ & 10 & $16 \mathrm{~h}$ & nein & 1 \\
\hline 7,10 & $\mathrm{w}$ & 2 & $37 \mathrm{~h}$ & nein & 1 \\
\hline 7,57 & $\mathrm{~m}$ & 7 & $76 \mathrm{~h}$ & nein & 3 \\
\hline
\end{tabular}

\subsubsection{Methanol}

Endogene Methanolspiegel liegen bei bis zu 2,0 mg/l im physiologischen Bereich. Bei elf Kindern war der Serummethanolspiegel geringgradig erhöht, darunter waren zehn SIDS-Fälle (Tabelle 66). 
Tabelle 66: Obduktionsfälle BMBF-Studie, Institutf. Rechtsmedizin München 19992001: SIDS-Fälle mit unphysiologisch hohen Serummethanolspiegeln

\begin{tabular}{|c|c|c|c|c|c|}
\hline $\begin{array}{c}\text { Methanol } \\
(\mathrm{mg} / \mathrm{l})\end{array}$ & Geschlecht & $\begin{array}{c}\text { Alter } \\
(\text { Monate })\end{array}$ & $\begin{array}{c}\text { postmortales } \\
\text { Intervall }\end{array}$ & Reanimation & $\begin{array}{c}\text { Ausbildungs- } \\
\text { niveau Eltern }\end{array}$ \\
\hline 2,46 & $\mathrm{~m}$ & 7 & 38 & nein & 3 \\
\hline 2,20 & $\mathrm{w}$ & 10 & 36 & nein & 3 \\
\hline 2,14 & $\mathrm{w}$ & 10 & 66 & nein & 1 \\
\hline 2,60 & $\mathrm{w}$ & 5 & 4 & nein & - \\
\hline 3,59 & $\mathrm{w}$ & 5 & 49 & nein & 2 \\
\hline 2,22 & $\mathrm{~m}$ & 9 & 29 & ja & 3 \\
\hline 3,69 & $\mathrm{w}$ & 6 & 63 & ja & - \\
\hline 2,36 & $\mathrm{w}$ & 7 & 68 & ja & - \\
\hline 2,04 & $\mathrm{~m}$ & 4 & 29 & ja & 3 \\
\hline 2,14 & $\mathrm{~m}$ & 7 & 76 & nein & 3 \\
\hline
\end{tabular}

\subsubsection{Aceton und Isopropanol}

Endogene Acetonspiegel liegen bei bis zu $6 \mathrm{mg} / 1$, ebenso wie Isopropanolspiegel [Gilg in Soyka 1995]. In drei Fällen fanden wir zu hohe Acetonspiegel, wobei es sich nur um einen SIDS-Fall handelte. Ein Fall wies erhöhte Isopropanolspiegel auf, dies war allerdings ein Non-SIDS-Fall, bei dem wir keinerlei Hinweise auf beispielsweise eine prämortale großflächige Hautdesinfektion o.ä. fanden (Tabelle 67).

Tabelle 67: Obduktionsfälle BMBF-Studie, Institut f. Rechtsmedizin München 19992001: SIDS-Fall mit unphysiologisch hohem Acetonspiegel

\begin{tabular}{|c|c|c|c|c|c|}
\hline $\begin{array}{c}\text { Aceton } \\
(\mathrm{mg} / \mathrm{l})\end{array}$ & Geschlecht & Alter & $\begin{array}{c}\text { postmortales } \\
\text { Intervall }\end{array}$ & Reanimation & $\begin{array}{c}\text { Ausbildungs- } \\
\text { niveau Eltern }\end{array}$ \\
\hline 10,0 & $\mathrm{~m}$ & 5 & 8 & nein & 1 \\
\hline
\end{tabular}

\subsubsection{Parallele Betrachtung der Begleitstoffanalysen}

Insgesamt wiesen 22 Kinder bei den Begleitstoffanalysen Auffälligkeiten auf (Tabelle 68), wobei sich keine Korrelationen zwischen den einzelnen Begleitstoffen ergaben und sich auch keine Unterscheide zwischen SIDS- und Non-SIDS-Fällen ergaben. 
Tabelle 68: Obduktionsfälle BMBF-Studie, Institut f. Rechtsmedizin München 19992001: Kinder mit erhöhten Alkoholspiegeln

\begin{tabular}{|c|c|c|c|c|c|}
\hline Fall & Methanol & Acetaldehyd & Aceton & Isopropanol & Ethanol \\
\hline SIDS & + & + & & & + \\
\hline SIDS & & & & & + \\
\hline SIDS & + & & & & \\
\hline SIDS & + & & & & \\
\hline SIDS & + & & & & \\
\hline SIDS & & + & & & + \\
\hline SIDS & & & & & + \\
\hline SIDS & & & & & \\
\hline SIDS & & + & & & \\
\hline SIDS & + & & & & \\
\hline SIDS & + & & & & \\
\hline SIDS & & + & & & + \\
\hline SIDS & + & & & & + \\
\hline SIDS & + & & + & & + \\
\hline SIDS & + & + & & & + \\
\hline SIDS & & & & & + \\
\hline SIDS & & & & & + \\
\hline Non-SIDS & + & & & & \\
\hline Non-SIDS & + & & & & \\
\hline Non-SIDS & & & & & \\
\hline Non-SIDS & & + & & & \\
\hline Non-SIDS & & & & & \\
\hline
\end{tabular}

Anzahl der Kinder mit einem erhöhten Begleitstoffspiegel: $\quad 16$

Anzahl der Kinder mit zwei erhöhten Begleitstoffspiegeln: 4

Anzahl der Kinder mit drei erhöhten Begleitstoffspiegeln: 3

\subsection{Die Non-SIDS-Fälle: Zusammenfassung}

Die Kinder der Kategorien 3 und 4 wurden durch umfangreiche Untersuchungen als Non-SIDS-Fälle identifiziert. Die Zuordnung zu den Kategorien 3 und 4 geht mit dem Nachweis einer sicheren (Kategorie 4) oder zumindest wahrscheinlichen (Kategorie 3) Todesursache einher. Diese Kinder kamen jedoch alle mit der Verdachtsdiagnose eines Plötzlichen Säuglingstodes zur Obduktion. 
Auch die Kinder, die wir in Kategorie 2 einordneten, und die in den vorhergehenden Auswertungen mit zu den SIDS-Fällen gezählt wurden, wiesen pathologische Befunde in mindestens einer der Untersuchungen auf. Allerdings sind keine dieser Befunde -nach heutigem Kenntnisstand- todesursächlich gewesen. Würde man jedoch der SIDSDefinition von Beckwith folgen (siehe Kapitel 1), so wären nur 5\% der von uns untersuchten Kinder SIDS-Fälle gewesen, 95\% hingegen würden nicht der SIDSDefinition entsprechen.

Fasst man die Besonderheiten der Non-SIDS-Fälle zusammen, so kommt man zu folgenden Erkenntnissen:

Unter Non-SIDS-Fällen waren die Jungen noch stärker überrepräsentiert als unter den SIDS-Fällen. Bei allen Tötungsopfern handelte es sich um Jungen. Das Alter der Kinder unterschied sich nicht auffällig von dem der SIDS-Fälle, die Auffindesituation war ähnlich, ebenso wie der Anteil von Frühgeburten. Die Non-SIDS-Fälle waren im Gegensatz zu den SIDS-Fällen bei der Geburt nie untergewichtig, beide Gruppen wissen die meisten Todesfälle aus augenscheinlich gesundem Zustand heraus in den Wintermonaten auf. Die Non-SIDS-Kinder waren doppelt so häufig erstgeborene Kinder wie die SIDS-Fälle, stammten eher aus unteren sozialen Schichten (Kategorie 2) als die SIDS-Fälle (Großteil aus Kategorie 3), sie wurden häufiger reanimiert (67\% versus 50\% bei SIDS-Fällen). In den Obduktionsbefunden unterschieden sich die beiden Gruppen durch häufigeres Auftreten von Lid- und Bindehautpetechien, Hirndruck, agonalem Erbrechen und pathologischen kardialen Befunden in der Non-SIDS-Gruppe. Alle anderen Befunde waren häufiger in der SIDS-Gruppe zu finden. Histologisch waren im Prinzip alle pathologischen Befunde häufiger bei den Non-SIDS-Fällen zu finden, die bakteriologischen Untersuchungen verliefen $\mathrm{zu}$ gleichen Anteilen positiv bezüglich pathogener Keime, nur in der virologischen Untersuchung waren die NonSIDS-Fälle wesentlich häufiger (29\%) auffällig als die SIDS-Kinder (8\%).

Bezüglich verschiedener Aspekte fanden sich also keine Unterschiede zwischen SIDSund Non-SIDS-Fällen, wobei uns leider gesunde Kontrollkinder als „neutrale“ Vergleichsgruppe fehlen. Viele Befunde jedoch sind auffällig, zeigen deutliche 
Differenzen zu den SIDS-Fällen. Die Wertigkeit dieser Besonderheiten wird im Kapitel 6.5. diskutiert. 


\section{TOTENSCHEINANALYSE}

\subsection{Allgemeine Analyse}

Wir haben im Studienzeitraum auch eine „Totenscheinanalyse“ an insgesamt 216 Todesbescheinigungen durchgeführt. Es handelte sich sowohl um Kinder aus der Studie, als auch um andere in München zwischen dem achten und 365. Lebenstag verstorbene Säuglinge.

Wir erhielten 70 Todesbescheinigungen mit Diagnosen, die einen Plötzlichen Säuglingstod möglich oder wahrscheinlich erscheinen ließen. Die Diagnosen waren dabei im einzelnen (Tabelle 69).

Tabelle 69: Analyse der Todesbescheinigungen der im Einzugsgebiet des Instituts $f$. Rechtsmedizin München zwischen 1999 und 2001 möglicherweise am „SIDS“ verstorbenen Säuglinge: Diagnose bezüglich Todesursache laut Todesbescheinigung

\begin{tabular}{|c|c|c|c|c|}
\cline { 2 - 5 } \multicolumn{1}{c|}{} & (V.a.) SIDS & (V.a.) Ersticken &,$? “ /$, ,unklar“ & $\begin{array}{c}\text { reflekt. Apnoe/ } \\
\text { Herzstillstand }\end{array}$ \\
\hline $\begin{array}{c}\text { Anzahl } \\
(n=70)\end{array}$ & 50 & 4 & 14 & 2 \\
\hline
\end{tabular}

Von diesen 70 Kindern konnten 49 obduziert werden, also 70\%. Bei 42 der obduzierten Kinder wurde tatsächlich die Diagnose „SIDS“ gestellt.

Bei 31 der 49 obduzierten Kinder enthielt die Todesbescheinigung die korrekte Diagnose, das entspricht 63\%, wobei Todesbescheinigungen mit Diagnosen bezüglich der Todesursache wie „?“, „unklar“ oder komplett fehlenden Angaben generell als falsch ausgefüllte Todesbescheinigungen gewertet wurden (Tabelle 70). 
Tabelle 70: Übersicht über die am Institut $f$. Rechtsmedizin von 1999-2001 durchgeführte Totenscheinanalyse

\begin{tabular}{|c|c|c|}
\cline { 2 - 3 } \multicolumn{1}{c|}{} & Anzahl (n) & Anzahl (in \%) \\
\hline $\begin{array}{c}\text { Anzahl möglicher } \\
\text { SIDS-Fälle }\end{array}$ & 70 & $100 \%$ \\
\hline davon obduziert & 49 & $70 \%$ \\
\hline $\begin{array}{c}\text { tatsächlich SIDS nach } \\
\text { Obduktion }\end{array}$ & 42 & $60 \%$ \\
\hline $\begin{array}{c}\text { richtige Diagnose laut } \\
\text { Todesbescheinigung }\end{array}$ & 31 & $44 \%$ \\
\hline
\end{tabular}

\subsection{Todesart laut Todesbescheinigung}

In den Todesbescheinigungen der in unsere Studie eingeschlossenen und untersuchten Kinder wurde von dem die Leichenschau durchführenden Arzt in 91\% der Fälle eine nicht aufgeklärte Todesart, in 6\% der Fälle eine nicht natürliche Todesart und in 3\% der Fälle eine natürliche Todesart bescheinigt (Tabelle 71).

Tabelle 71: Obduktionsfälle BMBF-Studie, Institut f. Rechtsmedizin München 19992001: Todesart laut Todesbescheinigung

\begin{tabular}{|c|c|c|c|}
\cline { 2 - 4 } \multicolumn{1}{c|}{} & $\begin{array}{c}\text { nicht aufgeklärte } \\
\text { Todesart }\end{array}$ & $\begin{array}{c}\text { nicht natürliche } \\
\text { Todesart }\end{array}$ & natürlicher Tod \\
\hline $\begin{array}{c}\text { Anzahl } \\
(n=72)\end{array}$ & $\begin{array}{c}66 \\
(91 \%)\end{array}$ & $\begin{array}{c}4 \\
(6 \%)\end{array}$ & 2 \\
$(3 \%)$
\end{tabular}

\subsection{Todesart laut Todesbescheinigung bezogen auf SIDS/ Non-SIDS}

Bei den nach Abschluß der Untersuchungen als SIDS-Fälle klassifizierten Kindern wurde von dem die Leichenschau durchführenden Arzt in 93\% der Fälle eine nichtaufgeklärte Todesart bescheinigt, bei den Non-SIDS-Fällen war dies in $89 \%$ der Fall (Tabelle 72). 
Tabelle 72: Obduktionsfälle BMBF-Studie, Institut f. Rechtsmedizin München 19992001: Todesart laut Todesbescheinigung bei SIDS- und Non-SIDS-Fällen

\begin{tabular}{|c|c|c|c|}
\cline { 2 - 4 } \multicolumn{1}{c|}{} & $\begin{array}{c}\text { nicht aufgeklärte } \\
\text { Todesart }\end{array}$ & $\begin{array}{c}\text { nicht natürliche } \\
\text { Todesart }\end{array}$ & natürliche Todesart \\
\hline SIDS & 41 & 2 & 1 \\
$(\mathrm{n}=44=100 \%)$ & $(93 \%)$ & $(5 \%)$ & $(2 \%)$ \\
\hline Non-SIDS & 23 & 2 & 1 \\
$(\mathrm{n}=26=100 \%)$ & $(89 \%)$ & $(8 \%)$ & $(3 \%)$ \\
\hline
\end{tabular}




\section{DISKUSSION}

\subsection{Diskussion der epidemiologischen Daten}

\subsubsection{Geschlecht}

Bei den von uns untersuchten SIDS-Fällen lag das Verhältnis von verstorbenen Mädchen zu Jungen bei 45\% zu 55\%. Daß Jungen häufiger am Plötzlichen Säuglingstod versterben als Mädchen, wird von vielen Autoren bestätigt: Jungen sind generell mit einem Anteil von 52\% bis 60\% gegenüber den Mädchen überrepräsentiert [Carpenter 1983; Wilske 1984; Taylor et al. 1995; Mitchell et al. 1997; Leach et al. 1999; Jorch 2000]. Das Risiko am Plötzlichen Kindstod zu versterben ist für Jungen gegenüber Mädchen laut verschiedenen Untersuchungen um das 1,41fache [Carpenter 1983], beziehungsweise um das 1,42fache [Mitchell et al. 1997] erhöht. In unserer Studie ergab sich für Jungen ein 1,2fach erhöhtes Risiko am Plötzlichen Kindstod zu versterben, es lag also etwas unter dem in der Literatur beschriebenen.

Wilske [1984] stellte fest, daß im ersten Lebenshalbjahr gehäuft Jungen verstarben (70\%), im zweiten Lebenshalbjahr hingegen mehr Mädchen (56\%). Diesen Befund können auch wir aus unseren Untersuchungen bestätigen: im ersten Lebenshalbjahr waren 64\% der Verstorbenen Jungen, im zweiten Lebenshalbjahr 60\% Mädchen. Kleemann [1996] konnte anhand seiner Untersuchungen diese Geschlechtsverteilung nicht bestätigen, das Geschlechtsverhältnis war in beiden Hälften des ersten Lebensjahres annähernd identisch.

Darüber, ob eine Jungenübersterblichkeit „SIDS-spezifisch“ ist oder nicht, finden sich in der Literatur gegensätzliche Meinungen: nach Jorch [2000] ist das Überwiegen des männlichen Geschlechts auch bei anderen Todesursachen im Säuglingsalter zu beobachten, nach Wilske [1984] ist die Jungenübersterblichkeit jedoch beim SIDS stärker ausgeprägt als bei anderen Todesursachen. Auch unter den von uns als NonSIDS-Fälle einzustufenden Kinder waren die Jungen mit $61 \%$ deutlich häufiger vertreten als Mädchen. 
Betrachtet man exemplarisch die Todesursachenstatistik des Statistischen Bundesamtes aus dem Jahr 1998, so zeigt sich, daß bei sämtlichen Todesursachen die Jungen, genau wie beim Plötzlichen Säuglingstod, überrepräsentiert sind, und zwar mit einem Anteil von $52 \%$ bis zu $73 \%$.

\subsubsection{Alter}

Bei den SIDS-Fällen dieser Studie fand sich ein Altersgipfel im dritten bis fünften Lebensmonat und ein weiterer im neunten Monat. Die Non-SIDS-Fälle zeigten eine etwas andere Altersverteilung, sie wiesen einen Sterblichkeitsgipfel im vierten und im zehnten Lebensmonat auf.

In der Literatur variieren die Angaben über bevorzugt betroffene Altersgruppen beim SIDS (Tabelle 73).

Tabelle 73: Altersgipfel beim Plötzlichen Säuglingstod laut verschiedenen Literaturangaben

\begin{tabular}{|c|c|c|c|c|c|c|c|}
\hline & \multicolumn{7}{|c|}{ Alter in Lebensmonaten } \\
\hline & 1 & 2 & 3 & 4 & 5 & 6 & 9 \\
\hline Eigene Untersuchungen & & & $\mathbf{x}$ & $\mathbf{x}$ & $\mathbf{x}$ & & $\mathbf{x}$ \\
\hline Althoff 1986 & & $\mathrm{x}$ & $\mathrm{x}$ & & & & \\
\hline Carpenter 1983 & & $\mathrm{x}$ & $\mathrm{x}$ & & & & \\
\hline Guntheroth 1995 & $\mathrm{x}$ & $\mathrm{x}$ & $\mathrm{x}$ & $\mathrm{X}$ & $\mathrm{x}$ & $\mathrm{x}$ & \\
\hline Jorch 2000 & & $\mathrm{x}$ & $\mathrm{x}$ & & & & \\
\hline Leach et al. 1999 & & $\mathrm{x}$ & $\mathrm{x}$ & $\mathrm{x}$ & & & \\
\hline Penning et al. 1996 & & $\mathrm{x}$ & $\mathrm{x}$ & $\mathrm{x}$ & & & \\
\hline Standfast et al. 1983 & & $\mathrm{x}$ & $\mathrm{x}$ & & & & \\
\hline Wilske 1984 & & $\mathrm{x}$ & $\mathrm{X}$ & & & & \\
\hline
\end{tabular}

Die in obiger Tabelle von allen Autoren beschriebene Häufung der SIDS-Fälle im zweiten Lebensmonat konnte in unserer Studie nicht beobachtet werden. Ebenso fand sich in der Literatur kein Hinweis auf die von uns beobachtete Häufung der Todesfälle im neunten Lebensmonat.

Die Altersverteilung, beziehungsweise die "Age at death curve" beim Plötzlichen Kindstod hat scheinbar eine einzigartige Charakteristik [Kraus 1983; Wilske 1984; 
Goldberg 1986; Emery 1983], die sich signifikant von über 20 anderen Todesarten unterscheidet. Sie ist unabhängig von anderen Risikofaktoren wie ethnischer Abstammung, Geschlecht, Geburtsgewicht, Jahreszeit, Alter der Mutter und Geburtsmonat [Goldberg et al. 1986].

Althoff [1986] erklärt diese besondere Häufung der SIDS-Fälle im zweiten und dritten Lebensmonat dadurch, daß in dieser Lebensphase die diaplazentar übertragenen Antikörper der Mutter weitgehend abgebaut werden. Da die Eigenproduktion des Säuglings diesen Abbau quantitativ und qualitativ noch nicht kompensieren kann, stehen dem Kind noch nicht genügend Immunglobuline für mögliche Infektionen zur Verfügung. Auch nach Bell et al. [1996] ist die Häufung der Todesfälle in einem bestimmten Lebensalter darauf zurückzuführen, daß das Kind in einem kleinen Zeitraum zwischen Verlust des mütterlichen Ig-G-Schutzes und dem Erwerb einer eigenen Immunität besonders wehrlos den unter Umständen fatalen Folgen einer Infektion mit toxinbildenden Bakterien wie Staphylokokken, Streptokokken und Enterobacter ausgeliefert ist.

\subsubsection{Verteilung der Todesfälle auf die Wochentage}

Wir fanden in dieser Studie eine Häufung von Dienstagen (20\%) und Samstagen (26\%) als Auffindetage des verstorbenen SIDS-Kindes, wobei dienstags mehr als doppelt so viele Mädchen verstarben wie an jedem anderen Tag (32\%), samstags hingegen mehr als doppelt so viele Jungen wie an den übrigen Wochentagen (32\%). Unter den NonSIDS-Fällen war kein spezieller Wochentag besonders häufig vertreten.

In der Literatur finden sich diskrepante Angaben über die Häufung von Todesfällen an bestimmten Wochentagen. In keiner Studie jedoch wurde eine Häufung von (weiblichen) Todesfällen an Dienstagen beobachtet, ebenso fand sich keine Erklärung, warum gerade Jungen gehäuft an Samstagen verstarben.

Auch in einer Studie von Kleemann [1996] verstarben samstags mehr Kinder (17\%) als an den übrigen Wochentagen. 
Laut Leach und Guntheroth gibt es gar keinen Wochentag mit signifikant erhöhter Anzahl von Todesfällen [Guntheroth 1995, Leach et al. 1999].

Öfter am Wochenende verstarben Säuglinge hingegen laut verschiedene anderen Autoren: Daltveit et al. [1998] fanden mehr Todesfälle an Sonntagen als an jedem anderen Tag. Besonders viele Todesfälle ereigneten sich an Wochenenden, wenn das Zimmer mit einem Elternteil geteilt wurde. Oft waren die Eltern zuvor auf einer Party, dadurch sei der sonst schützende Effekt des Schlafens im elterlichen Schlafzimmer reduziert worden. Eventuell besteht also ein Zusammenhang zwischen dem SIDS und den sozialen Aktivitäten, denen am Wochenende nachgegangen wird. Eine höhergradige Alkoholisierung der Eltern kann unbestritten $\mathrm{zu}$ einem akzidentellen Ersticken kleiner Kinder führen, wenn diese mit den Eltern ein Bett teilen [Kleemann 1996], doch liegen uns dazu in dieser Studie keine ausreichenden Daten vor.

Murphy et al. [1986] konnten an Untersuchungen von 6226 Fällen nachweisen, daß an den Wochenenden mehr Kinder verstarben als wochentags, wobei sie spekulierten, daß an Wochenenden die Eltern vielleicht mehr Hemmungen hätten, bei Auffälligkeiten des Kindes den ärztlichen Notdienst zu konsultieren.

Denkbar wäre aber auch, daß an Wochenenden eine spezialisierte kinderärztliche Betreuung schwerer erreichbar sein könnte als wochentags.

Mitchell et al. [1988] wiesen eine signifikant höhere Sterblichkeit an Wochenenden und in den Ferien nach.

Auch Lohmann [1996] und Williams et al. [1997] beobachteten eine erhöhte Sterblichkeit an Wochenenden, Spiers beobachtete, daß an normalen Arbeitstagen das SIDS-Risiko bei Nachkommen von Müttern mit weniger als zwölf Jahren Ausbildungsdauer 3,9 mal höher war als bei Kindern von Müttern mit mehr als sechzehnjähriger Ausbildungsdauer. An Wochenenden erhöhte sich das relative Risiko sogar auf 7,9. Möglicherweise hängt laut Spiers et al. das SIDS-Risiko mit der Sorgfalt bei der Beobachtung des Kindes zusammen. Diese wird am Wochenende oft anders organisiert als wochentags, wenn teilweise Tagesmütter in die Kinderbetreuung eingebunden werden. Möglicherweise haben Kinder von sozial unterprivilegierten Eltern deshalb am Wochenende ein erhöhtes SIDS-Risiko, weil sie unter der Woche, wenn die Eltern arbeiten, in einer Tagesstätte untergebracht werden. Dann dürften die Kinder von sozial gut gestellten Familien am Wochenende kein erhöhtes SIDS-Risiko 
haben, was Spiers Beobachtungen bezogen auf den Grad der Ausbildungsdauer entspricht [Spiers et al. 1999].

Alle diese Untersuchungen befassen sich jedoch mit dem Einfluß von Umweltfaktoren auf den Todeseintritt des Kindes an bestimmten Tagen, da nicht davon auszugehen ist, daß aufgrund natürlicher Ursachen Kinder häufiger an Wochenenden als wochentags versterben.

Die eigenen Untersuchungen können aufgrund der relativ geringen Fallzahl hier keine neuen Erkenntnisse liefern.

\subsubsection{Jahreszeitliche Verteilung}

In der internationalen Literatur finden sich keine einheitlichen Hinweise auf eine Häufung der Todesfälle in bestimmten Jahreszeiten, es zeigt sich lediglich eine steigende Tendenz zu den Herbst- und Wintermonaten hin, wie sie sich auch in dieser Studie zeigte: nach Jahreszeiten zusammengefaßt fanden sich bei den Mädchen die meisten Todesfälle im Herbst, bei den Jungen waren Sommer und Winter etwa in gleicher Häufigkeit vertreten. Insgesamt betrachtet waren der Herbst und Winter die Jahreszeiten mit den meisten Todesfällen. In den Sommermonaten verstarben die jüngsten Kinder (Durchschnittsalter 4,7 Monate), in den Herbstmonaten die ältesten (Durchschnittsalter 7,1 Monate).

In einer amerikanischen Studie an 190 SIDS-Fällen ergab sich eine Häufung der SIDSFälle im späten Herbst und Winter mit einem Gipfel im Januar, und ein Niedrigstand im Sommer in den Monaten Juli bis September [Standfast et al. 1983].

Lohmann [1996] stellte fest, daß in Niedersachsen zwischen 1985 und 1989 in den Monaten Oktober bis Januar überdurchschnittlich viele, in den Monaten Februar bis September unterdurchschnittlich viele Kinder am SIDS verstarben.

In einer Studie aus Großbritannien zeigte sich wiederum eine Abschwächung der Sterblichkeitsspitze in den Wintermonaten; jedoch ereignete sich noch immer mehr als ein Viertel der SIDS-Fälle in den drei Wintermonaten (27\%) [Leach 1999]. 
Wilske stellte bei geschlechtsbezogener Betrachtung der Verteilung der Todesfälle auf die Jahreszeiten fest, daß die Sterblichkeit bei den Jungen eine relativ konstante Verteilung über das gesamte Jahr mit Ausnahme der Monate April und September aufwies; bei den Mädchen ergab sich ein Häufigkeitsgipfel im Februar und März mit allmählicher Abnahme bis $\mathrm{zu}$ einer sommerlichen minimalen SIDS-Sterblichkeit [Wilske 1984].

Diese Beobachtung können wir aus unserem Untersuchungsgut nicht bestätigen: Bei den von uns untersuchten SIDS-Fälle ergab sich für die Jungen die höchste Sterblichkeit im Januar (16\%) und Juli (21\%), für die Mädchen im September (25\%).

Es wird beschrieben, daß Kinder mit einem Alter von mehr als 120 Tagen eher im Herbst und Winter verstarben, als jüngere Kinder [Kohlendorfer et al. 1998], was sich auch in unseren Untersuchungen annähernd so darstellte.

\subsubsection{Auffindesituation}

Die von uns untersuchten SIDS-Fälle wurden zu 67\% auf dem Bauch liegend tot aufgefunden. Zwischen Mädchen (Bauchlage bei 68\%) und Jungen (Bauchlage bei 67\%) fand sich kein Unterschied. Bei beiden Geschlechtern folgte der Bauchlage die Rückenlage (insgesamt 26\%) und die Seitenlage (insgesamt 7\%). Bei den Non-SIDSFällen fand sich eine ähnliche Verteilung. Die Beobachtung, daß mehr als die Hälfte aller am SIDS verstorbenen Kinder in Bauchlage tot aufgefunden wird, wird durch sämtliche SIDS-Studien bestätigt, gilt allerdings auch für unsere Non-SIDS-Fälle $(62 \%)$.

Kleemann et al. [1991] berichten über 87\% der Säugling in Bauchlage, 90\% der Kinder wurden nach Côté et al. [2000] auf dem Bauch gefunden. Nach Schlaud [1998] haben Kinder, die in Bauchlage schlafen, ein über dreifach erhöhtes SIDS-Risiko gegenüber Kindern, die nicht in Bauchlage schlafen. Laut Kleemann et al. [1995a] ist das Risiko drei- bis sechsfach erhöht. In Untersuchungen von Taylor et al. [1996] bevorzugten 57\% der am Plötzlichen Säuglingstod verstorbenen Kinder bereits zu Lebzeiten die Bauchlage, während es bei den Kontrollfällen nur 25\% waren. 
Laut Untersuchungen von Mitchell et al. trugen Kinder, die zum Schlafen auf den Rücken gelegt wurden, das geringste SIDS-Risiko. Kinder, die es gewohnt waren auf dem Bauch zu schlafen, hatten ein leicht erhöhtes SIDS-Risiko. Stark erhöht jedoch war das Risiko bei den Kindern, die es nicht gewöhnt waren, auf dem Bauch liegend zu schlafen, aber irgendwann trotzdem auf den Bauch gelegt wurden. Mitchell geht davon aus, daß die Fähigkeit eines in Bauchlage liegenden Kindes, sich aus einer potentiell lebensgefährlichen Situation zu befreien, bei einem "bauchlageunerfahrenen" Kind nicht so gut ausgebildet ist [Mitchell et al. 1999].

Auch nach Côté ist das "ungewohnte-auf-dem-Bauch-Schlafen" ein signifikanter Risikofaktor: 90\% der SIDS-Fälle wurden auf dem Bauch gefunden, davon waren 46\% nicht an die Bauchlage gewöhnt. Von diesen Kindern wurden 53\% von den Eltern ausnahmsweise auf den Bauch gelegt, 28\% hatten sich selbständig erstmalig auf den Bauch gedreht. $47 \%$ der von den von Eltern auf den Bauch gelegten Säuglinge verstarben schon während dem ersten oder zweiten Schlaf in Bauchlage [Côté et al. 2000].

Es gibt verschiedene Hypothesen darüber, warum die Bauchlage einen Risikofaktor für den Plötzlichen Säuglingstod darstellt. Bei erwachsenen Testpersonen mit einem respiratorischen Infekt wurde durch Nasenabstriche nachgewiesen, daß bei denjenigen, die auf dem Bauch lagen, die Nasenflora eine größere Anzahl von Mikroorganismen enthielt, als bei den Personen, die auf dem Rücken lagen. Diese Unterschiede waren schon nach einer Stunde in Bauch- beziehungsweise Rückenlage nachweisbar [Bell 1996]. Auch nach Rognum ist es denkbar, daß Säuglinge beim Schlafen in Gesichtslage das Risiko laufen, Sekrete mit erhöhtem Bakteriengehalt und Toxinproduktion in den oberen Luftwegen anzusammeln. Außerdem erschwert das Schlafen in Bauchlage die Regulation der Körpertemperatur, so daß es zu einem Wärmestau kommen könnte [Rognum 2000a]. Andere als SIDS-Ursachen diskutierte Faktoren im Zusammenhang mit der Bauchlage sind vasovagale Reaktionen, Kohlendioxid-Rückatmung, eine veränderte Gehirndurchblutung, positionsabhängige Asphyxie und reduzierte Aufwachreaktionen [Bell et al. 1996; Kenner et al. 2000].

Die Bauchlage scheint in Kombination mit anderen Risikofaktoren das SIDS-Risiko sogar zu potenzieren. Beispielsweise erhöht sich das SIDS-Risiko bei Kindern mit 
einem respiratorischen Infekt, die in Bauchlage schlafen, auf das zehnfache des normalen SIDS-Risikos [Ponsonby 1993].

\subsubsection{Schwangerschaftsdauer, Größe und Gewicht zum Zeitpunkt der Geburt und der Obduktion}

Von den SIDS-Fällen dieser Studie waren 19\% Frühgeburten, 13\% der Mädchen und 24\% der Jungen. Daß Frühgeburtlichkeit das SIDS-Risiko für ein Kind erhöht, wird von vielen Autoren bestätigt [Taylor et al. 1995; Leach et al. 1999; Platt et al. 2000]. Erstmals beschrieben wurde diese Entdeckung von Adelson und Kinney 1956, die unter ihren SIDS-Fällen bei 14\% Frühgeburtlichkeit feststellten, im Vergleich zu 8\% bei der Normalbevölkerung [Adelson et al. 1956].

Diese Frühgeburtlichkeit steht eng in Zusammenhang mit dem bei vielen SIDS-Fällen beobachteten zu niedrigen Geburtsgewicht. Ein niedriges Geburtsgewicht wird primär bei Frühgeburten beobachtet, kann aber auch durch andere Faktoren wie Rauchen, Mehrlingsschwangerschaften, Infektionskrankheiten der Mutter und kurze Intervalle zwischen Folgeschwangerschaften bedingt sein [Guntherroth 1995]. Bei der Geburt untergewichtige Kinder haben ein höheres Risiko, später an einem SIDS zu versterben [Taylor et al. 1995; Leach et al. 1999].

Im Jahr 1998 verstarben im ersten Lebensjahr in der Bundesrepublik Deutschland laut Statistischem Bundesamt 8\% der Kinder mit einem Geburtsgewicht von weniger als 2500 Gramm, aber nur 0,5\% mit einem Geburtsgewicht von 2500 Gramm oder mehr.

In unserem Untersuchungsgut waren bei der Geburt 17\% der SIDS-Kinder und keines der Non-SIDS-Kinder oberhalb der 3. Perzentile einzustufen, also für die vorhandene Körpergröße untergewichtig. Zum Obduktionszeitpunkt waren es schon 26\% der SIDSFälle und ebenfalls 26\% der Non-SIDS-Fälle. Es zeigte sich somit, daß die verstorbenen Kinder teilweise schon untergewichtig auf die Welt kamen, also aufgrund intrauteriner Entwicklungsdefizite, beziehungsweise sich nach der Geburt nicht ausreichend entwickelt haben und so zum Todeszeitpunkt untergewichtig waren.

Auch Platt et al. [2000] beschreiben, daß SIDS-Kinder signifikant zu leicht für ihr Gestationsalter sind. Valdes-Dapena beobachtete eine Wachstumsretardierung der Kinder, die später am SIDS verstorben sind, schon intrauterin, aber auch nach der 
Geburt. Die bisher unbekannte Ursache für den Plötzlichen Kindstod entwickle sich daher am ehesten schon intrauterin [Valdes-Dapena 1991]. Kleemann hingegen geht davon aus, daß nicht intrauterine Schädigungen oder Reifungs- beziehungsweise Wachstumssstörungen für den Plötzlichen Säuglingstod verantwortlich sind, da sich keine Erklärung dafür fände, daß im ersten Lebensmonat kaum Kinder am SIDS, dafür umso mehr im zweiten bis vierten Lebensmonat versterben [Kleemann 1996].

In Untersuchungen von Molz waren die Geburtsgewichte der SIDS-Fälle zum Zeitpunkt der Geburt eindeutig um die 50. Perzentile angeordnet, bei der Obduktion allerdings eher unter der 50. Perzentile. Insgesamt spricht dies für eine verzögerte Entwicklung der SIDS-Kinder als Gruppe. Dieselben Beobachtungen machte Molz für die Körperlänge und den Kopfumfang [Molz 1998].

\subsubsection{Gesundheitszustand vor Todeseintritt}

$38,8 \%$ der SIDS-Fälle unserer Studie und $48 \%$ der Non-SIDS-Fälle waren akut erkrankt, als sie verstarben, in aller Regel ohne daß die Erkrankung den Tod erklären könnte. Aus seit mindestens einer Woche völlig gesundem Zustand heraus verstarben $51 \%$ beziehungsweise $52 \%$ der Kinder.

Interessant erschien, daß unter den SIDS-Fällen besonders Jungen (fast doppelt so viele wie Mädchen) aus krankem Zustand heraus verstarben und daß von den "krank Verstorbenen" die meisten SIDS- und Non-SIDS-Fälle (37\% beziehungsweise 50\%) in den Wintermonaten verstarben.

Rognum et al. fassen zusammen, daß etwa in 50\% der SIDS-Fälle dem Tod leichte Infektionserkrankungen vorausgehen. Es wurde eine erhöhte SIDS-Inzidenz in Gebieten mit endemischer Häufung von Infektionskrankheiten beobachtet, ebenso wie ein Zusammenhang mit dem Auftreten von SIDS-Fällen und Keuchhustenepidemien beobachtet wurde [Rognum 2000a]. Auch laut Kleemann wiesen $40 \%$ bis $80 \%$ der SIDS-Fälle vor Todeseintritt Krankheitssymptome auf [Kleemann 1996].

So liegt also die Vermutung nahe, daß der Plötzliche Tod des Kindes in irgendeinem Zusammenhang mit einem infektiösen Geschehen steht, sei es daß der Infekt als einer 
von mehreren Trigger-Faktoren den Tod mitverursacht oder die Vulnerabilität des Kindes für einen anderen schädlichen Einflußfaktor erhöht.

\subsubsection{Geschwister}

SIDS-Fälle hatten meistens ein Geschwisterkind (54\%), dann folgten die Einzelkinder mit 20\%, gefolgt von den Kindern mit zwei (17\%), drei (7\%) oder mehr Geschwistern (2\%). Die Non-SIDS-Fälle zeigten eine etwas andere Verteilung: hier waren die Erstgeborenen am häufigsten betroffen, es folgten die Kinder mit zwei, drei, vier und mehr Geschwistern. Möglicherweise ist diese Beobachtung durch mangelnde, und mit Anzahl der Kinder zunehmende, Elternroutine bei erkranktem Kind zu erklären.

Laut Taylor et al. [1995] sind mehr als zwei vorhergehende Schwangerschaften ein Risikofaktor für das SIDS. Auch Daltveit et al. [1998] fanden ein höheres Risiko bei höherem Geburtenrang, und Lohmann [1996] beobachtete ein erhöhtes Risiko in Familien mit drei oder mehr Kindern, was mit dem beschriebenen erhöhten SIDSRisiko von Kindern junger Mütter schwer vereinbar ist.

Nach Spiers et al. [1993] hingegen hat das erstgeborene Kind das höchste SIDS-Risiko, welches dann kontinuierlich abnimmt, was wir auch in unseren Untersuchungen beobachten konnten.

Das Wiederholungsrisiko für Plötzliche Kindstode in Familien wird mit null- bis zehnfach angegeben, durchschnittlich wird für Folgekinder wohl ein zweifach erhöhtes SIDS-Risiko angenommen, wobei aufgrund fehlender Konkordanz bei Zwillingen nicht von genetischen Faktoren als Ursache auszugehen sei [Einspieler 2000].

Wie Poets zusammenfaßt, ist das Risiko für SIDS-Folgekinder zwar erhöht, jedoch nicht annähernd so hoch wie beispielsweise bei Kindern rauchender Mütter [Poets 2000].

$7 \%$ unserer SIDS-Fälle waren Zwillinge. In einem Fall verstarb auch das zweite Zwillingskind wenige Monate nach dem ersten an einem Plötzlichen Kindstod. Laut Beal ist ein zweiter SID bei dem überlebenden Zwilling selten (etwa 1\%). Anhand der Untersuchung von 637 Zwillingspaaren konnte bei 2\% ein simultaner Tod beider Zwillinge an einem Tag beobachtet werden. Einzeln geborene Kinder hatten in dieser 
Studie eine SIDS-Inzidenz von 2 pro 1000, Zwillinge hingegen von 3,7 pro 1000. In den Jahren 1986 bis 1988 stieg die Inzidenz bei Zwillingen sogar auf 9,8 pro 1000 an [Beal 1989]. Alle Daten sind jedoch vor dem Hintergrund möglicher unentdeckter Tötungsfälle durch sogenannte "weiche Bedeckung” kritisch zu interpretieren.

\subsubsection{Alter der Eltern bei der Geburt des verstorbenen Kindes}

Daß Kinder junger Mütter besonders häufig am SIDS versterben, ist eine oft beschriebene Beobachtung [Willinger et al. 1991; Valdes-Dapena et al. 1993; Taylor et al. 1995; Daltveit et al. 1998; Leach et al. 1999].

Auch in unseren Untersuchungen fand sich ein beachtlicher Unterschied des Alters der Mütter von SIDS-Kindern im Vergleich zum durchschnittlichen Alter der Mütter bei der Geburt eines Kindes.

Das durchschnittliche Alter der "SIDS-Mütter" lag trotz der vielen jungen Mütter dann doch bei 26,5 Jahren, das der "Durchschnittsmutter" bei 29,3 Jahren [Statistisches Bundesamt 2000]. In einer amerikanischen Studie hatten die SIDS-Mütter ein Durchschnittsalter von nur 21,4 Jahren [Lohmann 1996].

Jünger als zwanzig Jahre waren in unserem Untersuchungsgut knapp 25\% der Mütter, in der amerikanischen NICHD Studie waren 75\% der SIDS-Mütter bei der Geburt ihres ersten Kindes jünger als zwanzig Jahre [Hoffmann et al. 1988].

Daß junge Mütter unter den SIDS-Müttern deutlich überrepräsentiert wird, wird zwar durch sämtliche Studien, auch durch unsere Untersuchungen, bestätigt, jedoch findet sich nirgends eine Erklärung für dieses Phänomen. Auch über das Alter der „SIDSVäter" finden sich keine publizierten Untersuchungen.

\subsubsection{Soziale Stellung der Familie}

Die Einstufung der Familien in eine soziale Schicht bereitete Schwierigkeiten. Es stellte sich die Frage, wie man die soziale Situation einer Familie retrospektiv aus den vorhandenen Daten erfassen kann. Um eine Familie beispielsweise der Ober-, Mittel- 
oder Unterschicht zuordnen zu können, bräuchte man Angaben über Einkommen, Bildung, Anzahl der Kinder etc., die uns nicht zur Verfügung standen. Daher haben wir uns an anderen Studien orientiert, die das SIDS-Risiko in Verbindung mit der Ausbildungsdauer der Eltern, beziehungsweise der Ausbildungsdauer der Mutter untersuchten [Taylor et al. 1995, Lohmann 1996].

Uns lagen bezüglich der Berufstätigkeit der Eltern nur Angaben wie „Hausfrau“, „Schlosser“, „Lehrer“, „arbeitslos“ oder ähnliches vor. Daß beispielsweise der Status „Hausfrau“ weder Rückschlüsse auf die Ausbildung der Mutter noch auf den sozialen/ finanziellen Hintergrund der Familie zuläßt, ist offensichtlich. Ebenso kann es sich beim Arbeitslosen um einen arbeitslosen Hilfsarbeiter oder um einen Akademiker handeln, und es ist ebenfalls kein Rückschluß auf die soziale Stellung der Familie möglich. Alle nicht berufstätigen Eltern haben wir der Gruppe 1 zugeordnet; somit besitzt die Beurteilung dieser Gruppe nur geringen diagnostischen Wert bezüglich der sozialen Stellung der Familie. In den Gruppen 2 bis 4 dürfte sich eine solche Problematik nicht ergeben, da es sich hier um Elternteile handelte, von denen uns eine genaue berufliche Tätigkeit bekannt wurde.

Von den SIDS-Eltern unserer Untersuchung war der Großteil der Gruppe 3 (abgeschlossene Berufsausbildung) zuzuordnen, es folgten die problematische Gruppe 1 (nicht berufstätig), Gruppe 2 (Tätigkeiten ohne erfolgte Ausbildung) und Gruppe 4 (Akademiker).

In verschiedenen Studien stellt sich eine kurze Ausbildungsdauer der Mutter als SIDSRisikofaktor heraus: Weniger als zwölf Jahre Ausbildungsdauer der Mutter [Taylor et al. 1995] oder ein niedriges Bildungsniveau der Eltern [Arntzen et al. 1995; Daltveit et al. 1998] waren mit einem erhöhten SIDS-Risiko assoziiert, was aber auch sekundär dem jungen Alter der Mutter zuschreibbar sein kann.

Nach Untersuchungen von Leach waren unter den SIDS-Eltern häufiger sozial unterprivilegierte Gruppen vertreten als unter den Kontrollkindern [Leach et al. 1999]. Spiers beschreibt eine starke Assoziation zwischen dem Plötzlichen Kindstod und dem sozioökonomischen Status der Familie [Spiers et al. 1999].

Auch nach Arntzen et al. gibt es eine Assoziation zwischen dem Anteil der SIDS-Fälle an der erhöhten postneonatalen Mortalität und der niedrigen sozialen Schichtzugehörigkeit der Familie. Weiterhin war die SIDS-Mortalität bei den 
Erstgeborenen besonders hoch, wenn die Mütter jung waren, unverheiratet und ein niedriges Bildungsniveau hatten -voneinander sicherlich nicht unabhängige Faktoren-, wohingegen bei den Folgekindern die Mortalität nicht vom Alter und der Bildung der Mutter beeinflußt wurde. Eine solche Abhängigkeit fand sich bei den Non-SIDS-Fällen nicht [Arntzen et al. 1995]. Die Wohnsituation selbst ist nicht mit dem SIDS-Risiko assoziiert [Daltveit et al. 1998].

Hier sei noch einmal auf die Beobachtungen von Spiers verwiesen (siehe Kapitel 5.1.3.), der feststellte, daß das SIDS-Risiko bei Nachkommen von Müttern mit weniger als zwölf Jahren Ausbildungsdauer an Wochentagen 3,9 mal höher, an Wochenenden sogar 7,9 mal höher war als bei Müttern mit längerer Ausbildungsdauer. Die höhere Sterblichkeit an Wochentagen bei sozial schwächeren Müttern steht möglicherweise damit in Zusammenhang, daß Mütter aus höheren sozialen Schichten als Hausfrau tagsüber bei dem Kind bleiben können, während der Ehepartner arbeiten geht. Bei Frauen aus niedrigen sozialen Schichten müssen eher beide Ehepartner einer Erwerbstätigkeit nachgehen, so daß das Kind öfter in die Obhut einer Tagesmutter gegeben wird [Spiers et al. 1999].

Ein weiterer Versuch diese Beobachtungen zu erklären ist die Annahme, daß die Mutter über gewisse geistige Fähigkeiten verfügen muß, um Krankheitssymptome ihres Kindes bemerken und adäquat reagieren zu können [Guntheroth 1995], was möglicherweise bei Müttern mit längerer Ausbildungsdauer wahrscheinlicher erscheint.

\subsubsection{1. „Postvitales Intervall“ und Reanimation durch einen Notarzt}

Das Zeitintervall zwischen dem Moment, in dem das Kind letztmals lebend gesehen wurde und dem, in dem es tot aufgefunden wurde, lag bei unseren Fällen in $6 \%$ bei unter einer Stunde. Kleemann berichtet ebenfalls von einem postvitalen Intervall von weniger als einer Stunde bei 6\% der Fälle [Kleemann et al. 1991].

Mehr als zehn Stunden Zeit, um den Tod des Kindes festzustellen, brauchten in unserer Untersuchung 9\% der Eltern, in Untersuchungen von Kleemann war dies bei $17 \%$ der Fall, wobei es sich sozial meist um schlechte Verhältnisse handelte [Kleemann et al. 1991]. 
Es fiel auf, daß bei unseren SIDS-Fällen die verstorbenen Jungen fast doppelt so häufig (82\%) innerhalb der ersten fünf Stunden nach möglichem Todeseinritt aufgefunden wurden wie Mädchen (45\%). In der Literatur findet sich kein Anhalt für eine Erklärung dieser Beobachtung. Vielleicht sind Jungen umsorgtere Kinder, weil eventuell mehr erwünscht oder weil vielleicht weinerlicher und mehr Pflege beanspruchend? Bei den Non-SIDS-Fällen ergab sich ein solch deutlicher Unterschied allerdings nicht (Mädchen: 57\%, Jungen 67\%).

Die Tatsache, daß Jungen schneller aufgefunden wurden als Mädchen, könnte vielleicht ein Hinweis darauf sein, warum Jungen häufiger reanimiert wurden (Mädchen: 47\%, Jungen: 53\%): Bei einem kürzeren postvitalen Intervall sind möglicherweise die Todeszeichen noch nicht in voller Ausprägung sichtbar gewesen. Bei den Non-SIDSFällen war die Differenz der Reanimationsraten beider Geschlechter noch deutlicher ausgeprägt: $43 \%$ der Mädchen aber doppelt so viele Jungen (82\%) wurden reanimiert, was wiederum nicht mit dem bei den Non-SIDS-Fällen annähernd gleich langen postvitalen Intervall bei Jungen und Mädchen in Einklang zu bringen ist, so daß keine Erklärung für diese Beobachtungen geliefert werden kann.

\subsection{Diskussion der makroskopischen und mikroskopischen Befunde}

\subsubsection{Petechien}

Der häufigste bei der Obduktion zu erhebende Befund waren Petechien. Petechien werden in der Literatur als charakteristischer Befund beim Plötzlichen Säuglingstod beschrieben [Valdes-Dapena et al. 1993; Kleemann et al 1997a]. Primär beobachtet werden sie bei etwa $85 \%$ der SIDS-Fälle als thorakale Petechien an Thymus, subpleural und subepikardial [Kleemann et al 2000]. Natürlich kommen Petechien auch an anderen Stellen beziehungsweise Organen vor, wie im Perikard, an der parietalen Pleura oder aber an den Lid- und Bindehäuten oder anderen Hautregionen, wobei sie dort nicht als SIDS-typisch anzusehen sind, sondern möglicherweise als Hinweis auf einen Erstickungvorgang zu deuten sind. Laut Risse sind jedoch die Petechien beim Ersticken 
eher in geringerer Anzahl vorhanden und kleiner, beim SIDS hingegen eher prominent und zahlreich [Risse 1989a].

Die SIDS-Fälle unserer Untersuchungen wiesen in $84 \%$ Thymus-Petechien auf, unter den Non-SIDS-Fällen war dies in 61\% der Fall (Tabelle 74).

Tabelle 74: Angaben zur Häufigkeit von Thymus-Petechien in der Literatur und im eigenen Untersuchungen im Rahmen der BMBF-Studie

\begin{tabular}{|l|c|c|}
\cline { 2 - 3 } \multicolumn{1}{c|}{} & SIDS & Non-SIDS \\
\hline Eigene Untersuchungen & $\mathbf{8 4 \%}$ & $\mathbf{6 1 \%}$ \\
\hline Borhani et al. (1973) & $42 \%$ & - \\
\hline Kleemann et al. (1995b) & $76 \%$ & $33 \%$ \\
\hline Risse et al. (1989a) & $87 \%$ & - \\
\hline Valdes-Dapena (1991) & $44 \%$ & $25 \%$ \\
\hline Wilske (1984) & $79 \%$ & - \\
\hline
\end{tabular}

Bezüglich der Häufigkeit dieser Petechien bei den von uns untersuchten Kindern war zu beobachten, daß sich sowohl bei SIDS-Fällen, als auch bei Kindern der Kategorien 3 oder 4 alle Abstufungen von „keine“ bis zu „massenhaft“ fanden. Die Kinder der Kategorie 1 wiesen wenige bis mittelmäßig viele auf, die Kinder der Kategorie 2 meistens (60\%) eine mittlere Anzahl, Kinder aus Kategorie drei ebenfalls meistens (64\%) mäßig viele und die Kinder aus Kategorie vier wiesen zu gleichen Anteilen (jeweils 25\%) alle Abstufungen von „keine“ bis „massenhaft“ auf. Somit können wir aus unseren Untersuchungen nicht bestätigen, daß es sich bei dieser Art von Petechien um einen SIDS-typischen Befund handelt.

Von verschiedenen Autoren wird eine Häufung der Petechien im intrathorakalen Thymusanteil beschrieben, der zervikale Anteil ist häufig ausgespart oder nur in reduzierter Anzahl von Petechien bedeckt [Valdes-Dapena 1993, Kleemann 1996, Beckwith 1988]. Ursächlich für die Entstehung von Petechien könnte daher nach Beckwith ein negativer intrathorakaler Druck sein, welcher verursacht wird durch obstruktive Apnoen und die daraus resultierenden verstärkten Inspirationsanstrengungen des Kindes [Beckwith 1988]. Laut Krous spricht die Tendenz, Petechien gehäuft im Cortex und nicht im Mark des Thymus zu finden, ebenfalls für eine Verursachung der 
Blutungen durch obstruktive Apnoen [Risse 1989a], diese Theorie wird jedoch unter anderem von Kleemann und Valdes-Dapena abgelehnt [Valdes-Dapena 1991, Kleemann 1996].

Kleemann faßt zusammen, daß Thymus-Petechien nicht durch das postmortale Intervall beeinflußt sind, keine Lageabhängigkeit aufweisen, nicht durch Reanimationsmaßnahmen herbeigeführt werden und wie subpleurale Petechien eine Altersabhängigkeit aufweisen, wobei bei älteren Kindern eine höhere Dichte beobachtet wurde [Kleemann et al 1995b und 1997a].

Subepikardiale Petechien waren der zweithäufigste Obduktionsbefund in dieser Studie. In der Literatur finden sich folgende Angaben über deren Häufigkeit (Tabelle 75):

Tabelle 75: Angaben zur Häufigkeit subepikardialer Petechien in der Literatur und im eigenen Untersuchungsgut im Rahmen der BMBF-Studie

\begin{tabular}{|l|c|c|}
\cline { 2 - 3 } \multicolumn{1}{c|}{} & SIDS & Non-SIDS \\
\hline Eigene Untersuchungen & $\mathbf{6 1 \%}$ & $\mathbf{4 3 \%}$ \\
\hline Beckwith (1970) & $77 \%$ & $40 \%$ \\
\hline Borhani et al. (1973) & $47 \%$ & - \\
\hline Kariks (1988) & $88 \%$ & - \\
\hline Kleemann (1995b) & $66 \%$ & $29 \%$ \\
\hline Krous et al. (1984) & $70 \%$ & - \\
\hline Valdes-Dapena et al. (1993) & $46 \%$ & $23 \%$ \\
\hline
\end{tabular}

Demnach waren subepikardiale Petechien durchschnittlich bei $65 \%$ der untersuchten SIDS-Fälle vorhanden, wie auch annähernd in dieser Studie. Kleemann beschreibt, daß die Kinder bei denen eine besonders hohe Dichte an subepikardialen Petechien nachgewiesen wurde, signifikant älter waren, als die bei denen man nur wenige oder keine fand. Er schreibt diese Beobachtung der Tatsache zu, daß in diesen Fällen eine längere Streßphase mit massiver Katecholaminausschüttung vorausgegangen sei [Kleemann 1995b].

Subpleurale Petechien schließlich waren der dritthäufigste Befund bei der Obduktion, sie waren makroskopisch in $45 \%$ der SIDS-Fälle und $29 \%$ der Non-SIDS-Fälle nachweisbar (Tabelle 76). 
Tabelle 76: Angaben zur Häufigkeit subpleuraler Petechien in der Literatur und im eigenen Untersuchungsgut im Rahmen der BMBF-Studie

\begin{tabular}{|l|c|c|}
\cline { 2 - 3 } \multicolumn{1}{c|}{} & SIDS & Non-SIDS \\
\hline Eigene Untersuchungen & $\mathbf{4 5 \%}$ & $\mathbf{2 9 \%}$ \\
\hline Beckwith (1970) & $82 \%$ & $34 \%$ \\
\hline Borhani et al. (1973) & $89 \%$ & - \\
\hline Kleemann (1996) & $61 \%$ & $28 \%$ \\
\hline Krous (1984) & $85 \%$ & - \\
\hline
\end{tabular}

[modifiziert nach Kleemann 1996]

Sie scheinen im Gegensatz zu subepikardialen und Thymus-Petechien altersunabhängig aufzutreten, was möglicherweise auf einen unterschiedlichen Entstehungsmechanismus zurückzuführen ist. Intrathorakale Petechien sind nach Kleemann im Zusammenhang mit anderen SIDS-typischen Obduktionsbefunden ein Beleg für eine länger bestehende respiratorische Insuffizienz mit Hypoxie bei vermehrter Atemarbeit bis zur Schnappatmung bei persistierendem Kreislauf. Sie sind zwar ein typischer Obduktionsbefund bei Plötzlichen Kindstodfällen, jedoch nicht für diese spezifisch, da sie auch bei anderen Todesarten im Kleinkindesalter nachweisbar sind [Kleemann et al. 2000].

Schließlich sei noch auf die in dieser Studie bei $24 \%$ der SIDS-Fälle und $43 \%$ der NonSIDS-Fälle aufgetretenen Petechien in den Lid- und Bindehäuten eingegangen. Alle vier in dieser Studie aufgedeckten Fälle tödlicher Kindesmißhandlungen wiesen Petechien im Lid- und Bindehautbereich auf, wobei es sich um drei typische „battered child“Fälle, davon jedoch eine Tötung durch „,weiche Bedeckung“, und ein Schütteltrauma handelte.

Die anderen Kinder mit dieser Art von Petechien waren an schweren Infekten (fünf Kinder) oder einem Waterhouse-Friderichsen-Syndrom verstorben. Aber auch fast ein Viertel der als SIDS-Fälle einzuordnenden Kinder wies (einzelne) konjunktivale Petechien auf, obwohl diese als SIDS-untypisch beschrieben werden [Risse 1989a; Kleemann et al. 2000], wobei anzumerken ist, dass wir in jedem Fall eine OperationsStereo-Lupe verwendet haben. In einem Fall zeigte ein Säugling unzählige Petechien an Lid- und Bindehäuten, in den Augenwinkeln, der Jochbeinregion, Vorder- und Hinterohrregion, Halshaut, Nackenhaut, am Brustkorb und den Oberarmen. Bei negativen bakteriologischen Befunden fand sich in der histologischen Untersuchung 
eine Bronchopneumonie mit Tracheitis und Laryngitis, die als Todesursache ausreichend erschien.

Konjunktivale Petechien sind zwar kein Beweis für eine Strangulation, sollten aber zu einer detaillierten Untersuchung führen [Kleemann 1995b].

\subsubsection{Respirationstrakt}

Insgesamt fanden sich bei den SIDS-Fällen in dieser Studie häufig vergrößerte Halslymphknoten, gerötete Luftwege, makroskopisch „gummiartige Beschaffenheit“ der Lungen (schlechte Belüftung), Lungenödem, Lungenstauung und intraalveoläre Makrophagenvermehrungen. Ein häufiger Befund bei den SIDS-Kindern waren somit makroskopisch und/ oder mikroskopisch nachweisbare leichte Infekte besonders der oberen Atemwege, was als durchaus SIDS-typisch angesehen wird [Kleemann 2000]. Aoki konnte bei $30 \%$ der von ihm untersuchten SIDS-Fälle respiratorische Infekte nachweisen [Aoki 1994]. Es handelte sich fast ausschließlich um lymphozytäre Infiltrate, so daß ein Zusammenhang mit einem viralen Infekt denkbar erscheint. In Untersuchungen von Althoff hatten 35\% der SIDS-Fälle herdförmig lokalisierte, überwiegend monozytäre Zellinfiltrate außerhalb der ortsständigen lymphatischen Strukturen. Eine generalisierte Rhinopharyngitis war bei $40 \%$ der SIDS-Fälle nachweisbar, jedoch ohne entsprechende vorbestehende Krankheitssymptome [Althoff 1986].

Es zeigt sich, daß in dieser Studie bei 35\% der SIDS- und Non-SIDS-Fälle bereits bei der Obduktion ein Atemwegsinfekt festgestellt wurde, der auch histologisch bestätigt werden konnte. In 39\% der Fälle wurde makroskopisch kein Anhalt für einen Atemwegsinfekt gefunden, mikroskopisch fanden sich jedoch pathologische Befunde, teilweise sogar ausgeprägte (Broncho-)Pneumonien. In keinem Fall konnte makroskopisch eine Pneumonie sicher diagnostiziert werden, mikroskopisch fanden sich aber bei mehr als einem Viertel der Kinder (26\%) zumindest leichte Pneumonien. Nach Valdes-Dapena ist es sogar für Kinderpathologen fast unmöglich, makroskopisch bei Lungen dieser Größe eine Pneumonie richtig zu diagnostizieren [Valdes-Dapena et al. 1993]. 
Die Beurteilung des Schweregrads einer Pneumonie ist eine der schwierigsten Aufgaben bei der Diagnosestellung. Es handelt sich immer um eine subjektive Einschätzung (in stärkere oder weniger starke Ausdehnung) und es gibt keinen pathologischen Standard um klassifizieren zu können, ob die Ausprägung stark genug ist, um den Tod erklären zu können [Aoki 1994].

Die Beobachtung, daß bei SIDS-Fällen häufig leichte Atemwegsinfekte vorliegen, wurde in vielen anderen Studien bestätigt. Valdes-Dapena berichtet vom häufigen Auftreten von Laryngitiden in milder bis gemäßigter, akuter oder chronischer Form. Entzündungen der Mukosa von Epiglottis, Larynx und Trachea sind bei einem Drittel der SIDS-Fälle nachweisbar [Valdes-Dapena et al.1993]. Nach Untersuchungen von Aoki wiesen 21\% der SIDS-Fälle eine Tracheitis, $8 \%$ eine Bronchitis und 7\% eine Pneumonie auf [Aoki 1994].

Die Bedeutung der Tatsache, daß bei solch hoher Anzahl Atemwegsinfekte bei SIDSFällen nachgewiesen werden können, besteht vielleicht darin, daß diese Infekte oft in einem Alter auftreten, in dem der kindliche Organismus noch nicht die Fähigkeit erlangt hat, Infektionen lokal zu begrenzen, sondern systemisch reagiert. Daher unterscheiden sich die Krankheitsverläufe bei Säuglingen von denen bei Erwachsenen. Da sich jedoch in verschiedenen Untersuchungen kein signifikanter Unterschied bezüglich der Ausprägung dieser Entzündungen bei SIDS-Fällen und Kontrollfällen zeigen ließ, ist davon auszugehen, daß es sich um eine für das Alter physiologische Reaktion handelt und daß diese Befunde bei einem Großteil der SIDS-Fälle keine todesursächliche Bedeutung haben. Da aber bereits eine geringe Schleimhautschwellung beispielsweise im Larynx, der die engste Stelle des kindlichen oberen Respirationstraktes darstellt, zu einer massiven Reduktion des Lichtungsquerschnittes führen kann, sind solche „physiologischen Entzündungen“ durchaus als Triggermechanismen bei gleichzeitig bestehenden ungünstigen äußeren Faktoren, wie zum Beispiel die Bauchlage, vorstellbar. Eine Schleimhautschwellung im Larynx von nur einem Millimeter reduziert den Lichtungsquerschnitt bereits um 50\% [Kleemann 2000].

Nach Rognum reagiert ein großer Teil der SIDS-Opfer in abnormer Weise auf einen harmlosen Infekt und es wird ein Todesmechanismus ausgelöst, der einem septischen Schock ähnelt [Rognum 2000]. 
Typische Befunde an den Lungen von SIDS-Fällen nichtentzündlicher Genese sind Blutstauung, Lungenödem und Dystelektasen.

Gestaute Kapillaren im Lungengewebe fanden sich in dieser Studie bei 86\% der SIDSFälle und 68\% der Non-SIDS-Fälle. Teilweise kam es zu Hämorrhagien, und zwar bei $45 \%$ der SIDS-Fälle und 35\% der Non-SIDS-Fälle. Laut Valdes-Dapena ist die Blutstauung in den erweiterten Alveolarkapillaren oft eindrucksvoll ausgeprägt und kann verdickte Alveolarsepten vortäuschen. Bei fast $90 \%$ der SIDS-Fälle ihrer Untersuchungen war eine solche Stauung nachweisbar. Trotzdem ist diese nicht SIDSspezifisch, denn auch unter den geklärten Todesfällen war eine Lungenstauung bei über $80 \%$ der Kinder nachweisbar. Bei ausgeprägter Lungenstauung kommt es zu einer alveolären Hämorrhagie, die vermutlich agonal oder sogar noch postmortal entsteht und bei $66 \%$ der SIDS-Fälle ihrer Untersuchungen nachweisbar war [Valdes-Dapena et al.1993]. Aoki fand bei $83 \%$ der SIDS-Fälle und bei $72 \%$ der Kontrollfälle eine Lungenstauung, mit teilweise (25\%) einer großen Menge extravasaler Erythrozyten intraalveolär [Aoki 1994].

Ein intraalveoläres Lungenödem fand sich in unserer Studie bei 57\% der SIDS-Fälle und $54 \%$ der Non-SIDS-Fälle. Es wird ebenfalls als agonales Ereignis oder sogar postmortales Artefakt angesehen und findet sich sowohl bei SIDS-Fällen als auch bei Kontrollen [Ogbuihi et al. 1988; Valdes-Dapena 1991; Valdes-Dapena et al.1993]. Lungenödem und Stauung sind zwar oft sehr deutlich ausgeprägt, haben aber nur geringe Aussagekraft, weil sie nur eine Hypoxämie in der agonalen Phase reflektieren, oder sogar als postmortales Artefakt angesehen werden müssen und nur von sehr geringem diagnostischen Wert sind [Aoki 1994].

Die intraalveoläre Deckzelldesquamation erschien zum Teil sehr eindrucksvoll, da diese in einzelnen Fällen fast den kompletten Alveolarraum ausfüllten. Meistens waren sie aber weniger stark ausgeprägt. Insgesamt konnten wir eine Alveolardeckzelldesquamation bei $37 \%$ der SIDS-Fälle und bei $32 \%$ der Non-SIDSFälle nachweisen. Auch Kleemann berichtet von der häufigen Beobachtung abgelöster ein- oder mehrzelliger Alveolarmakrophagen, die in einigen Fällen die Alveolen regelrecht ausfüllten. Von der Annahme, es handle sich um eine Infektionsfolge 
(Desquamationspneumonie, Alveolardeckzellenkatarrh), sei man jedoch inzwischen abgekommen [Kleemann 2000].

Emphysematische Überblähung der Alveolen im Kombination mit dys- oder atelektatischen Bezirken fanden sich in dieser Studie bei $41 \%$ der SIDS-Fälle und $32 \%$ der Non-SIDS-Fälle. Atelektasen und Emphysem werden oft gemeinsam gefunden und sind oft durch Reanimation verursacht [Valdes-Dapena 1993, Aoki 1994].

\subsubsection{Kongenitale Fehlbildungen}

In dieser Studie fanden sich bei sieben (14\%) der als SIDS-Fälle eingestuften Kinder Fehlbildungen oder Normvarianten ohne relevanten Krankheitswert: ein Pierre-RobinSyndrom, ein Vorhof-Septum-Defekt, beidseits zweigelappte Lungen, Verlauf der Vena subclavia über den Thymus hinweg mit isthmusartiger Einengung an dieser Stelle, offenes Foramen ovale, an drei Stellen nicht gedecktes Foramen ovale bei allgemein vergrößertem Herzen und eine Ausweitung der Aorta ascendens mit Narbenbildung in diesem Bereich.

SIDS-Fälle hatten in verschiedenen Untersuchungen nachweislich mehr angeborene Fehlbildungen als das durchschnittliche Kind [Leach et al. 1999]. Molz et al. wiesen bei SIDS-Kinder vier mal so viele anatomisch von der Norm abweichende Varianten nach, wie bei Non-SIDS-Kindern [Molz et al. 1992], und auch Platt et al. bestätigten, daß signifikant mehr SIDS-Fälle als Non-SIDS-Fälle Fehlbildungen aufwiesen, wobei es sich in einem Drittel davon um kardiovaskuläre Anomalien handelte [Platt et al. 2000].

\subsection{Diskussion der mikrobiologischen Befunde}

Der häufigste bei den SIDS-Fällen dieser Studie nachweisbare Erreger war Staphylokokkus aureus (20\%), gefolgt von Streptokokken bei 8\% der Fälle.

Telford et al. wiesen in einer Studie an 46 SIDS- und 46 Non-SIDS-Fällen an nasopharyngealen Abstrichen eine dichtere Besiedlung mit Staphylokokkus aureus, Streptokokken und Enterobacter bei den SIDS-Fällen nach [Telford et al. 1989]. Häufig 
diskutiert wird die Bedeutung einer Infektion mit Clostridium botulinum für den Plötzlichen Kindstod. In dieser Studie jedoch erfolgte keine Untersuchung auf Botulismus.

Bezüglich der Bedeutung der bakteriologischen Untersuchung in dieser Studie läßt sich feststellen, daß in vielen Fällen der bakteriologische und der mikroskopische/ makroskopische Befund diskrepant waren: Es lagen insgesamt aus der SIDS- und NonSIDS-Gruppe fünf Fälle mit der Enddiagnose „Sepsis“ vor. In drei Fällen war das Kind auch anamnestisch leicht erkrankt gewesen, bei zwei Fällen hatten die Eltern jedoch das Kind als völlig gesund eingeschätzt. Die makroskopischen Befunde waren nicht sehr beeindruckend, und auch in der histologischen Untersuchung erschien nur ein Kind schwer krank mit Pneumonie, Nephritis, Hepatitis und gering ausgeprägter Myokarditis. Der Vollständigkeit halber sei erwähnt, daß dieses Kind zu Lebzeiten zusätzlich an einem AGS (adrenogenitales Syndrom) in Kombination mit einem MCAD-Defekt (Mittelketten-Acyl-Coenzym-A-Dehydrogenase-Defekt) gelitten hatte. Aber eben dieses Kind mit den gravierenden histologischen Befunden wies makroskopisch bei der Obduktion keine Entzündungszeichen außer vergrößerten Lymphknoten auf.

Zwischen den Ergebnissen der verschiedenen Untersuchungen mußte immer abgewogen werden, um zu einer endgültigen Diagnose zu gelangen. Nur selten bestätigten sich der mikroskopische/ makroskopische und der bakteriologische Befund gegenseitig. Etwa 76\% der SIDS-Fälle dieser Studie wiesen histologisch wenigstens leichte Anzeichen für einen Atemwegsinfekt auf, aber nur in 33\% der Fälle war auch bakteriologisch ein Erreger nachweisbar. Bei den Non-SIDS-Fällen waren histologisch sogar in $82 \%$ der Fälle Atemwegsveränderungen nachweisbar, in der bakteriologischen Untersuchung erfolgte nur bei 39\% ein positiver Erregernachweis.

Im Allgemeinen wird den bakteriellen Infektionen aber für die Ätiologie des SIDS eine wesentlich geringere Rolle beigemessen als den viralen Infekten [Guntheroth 1995].

Daß virale Infektionen in bisher ungeklärter Weise mit dem Plötzlichen Säuglingstod in Verbindung zu stehen scheinen, ergibt sich unter anderem aus der Tatsache, daß sich eine Häufung der Todesfälle in den Herbst- und Wintermonaten zeigt. Sie zeigen aber nur zum Teil zeitliche Übereinstimmung mit viralen Epidemien, wie beispielsweise dem 
Keuchhusten [Rognum 2000a]. Von Bonser et al. wurde eine Häufung von SIDS-Fällen während Influenza A-Epidemien beschrieben [Bonser et al. 1978]. Am häufigsten wurden bei SIDS-Fällen Influenza-, RSV-, Rhino-, Rota- und Adenoviren nachgewiesen, und zwar in etwa 25\% der Fälle [Kurz 2000]. Auch die Tatsache, daß wir fast nur lymphozytäre Entzündungsreaktionen nachweisen konnten, spricht verstärkt für eine virale Genese.

Insgesamt erfolgte bei 18\% der SIDS-Fälle und 32\% der Non-SIDS-Fälle dieser Studie ein positiver Virusnachweis. Wie oben bereits erwähnt, wiesen aber $76 \%$ der SIDSFälle einen Atemwegsinfekt auf. Es gab drei Fälle mit gleichzeitiger bakterieller und viraler Infektion: Klebsiella pneumoniae mit Adenoviren, Pneumokokken mit Parainfluenza-3-Viren und Pneumokokken mit Zytomegalieviren. Es wurden in unserer Studie von den asservierten Materialien bisher leider nur die Trachealabstriche und Stuhlproben virologisch untersucht. Nach diesen Ergebnissen scheint jedoch die Notwendigkeit einer Untersuchung der anderen asservierten Proben, wie beispielsweise Herzblut, Milzabstrich oder Liquor, für eine sicherere Diagnosestellung unabdingbar. Auch Perikardflüssigkeit hat sich praktisch für den Nachweis kardiotroper Viren bewährt.

In unserem Untersuchungsgut wiesen 9,6\% der SIDS-Fälle im Trachealabstrich eine Infektion mit dem Zytomegalievirus (ZMV) als häufigstem viralen Erreger auf. Cremer und Althoff konnten in eigenen Untersuchungen der Glandula parotidea von 122 SIDSFällen bei 5\% eine ZMV-Infektion nachweisen [Cremer et al. 1991]. Püschel und Mitarbeitern [Püschel et al. 1988] gelang dieser Nachweis bei 7\% der von ihnen untersuchten SIDS-Fälle. Molz und Mitarbeiter [Molz et al. 1985] stellten in ihrem Untersuchungsgut eine ZMV-Inzidenz von 10\% unter den SIDS-Fällen fest. Allerdings erfolgte in allen diesen Untersuchungen der Virus-Nachweis direkt an den meist in Mitleidenschaft gezogenen Parotiden. Laut Cremer und Althoff findet sich beim SIDS oft eine unspezifische Parotitis, bei der ein ZMV-Nachweis auch bei aufwendiger Diagnostik nicht gelingt [Cremer et al. 1991].

Bei den SIDS-Fällen dieser Studie konnten wir zwar histologisch bei 16\% eine unspezifische Entzündungsreaktion in den Parotiden nachweisen, jedoch war nur bei einem der acht SIDS-Fälle mit Sialadenitis auch eine Zytomegalie-Infektion 
nachweisbar. Bei den Non-SIDS-Fällen hingegen wiesen fünf Kinder eine Sialadenitis auf, hierbei konnte in drei Fällen das Virus nachgewiesen werden, womit sich bei den Non-SIDS-Fällen eine Übereinstimmung in 60\% der Fälle ergab.

Ein Zusammenhang des SIDS mit Pertussis-Infektionen konnte am hiesigen Institut bereits früher im Rahmen einer Multi-Center-Studie ausgeschlossen werden [Heininger et al. 1996].

\subsection{Diskussion der toxikologischen Befunde, der Ethanolbestimmung und der Begleitstoffanalyse}

In der toxikologischen Untersuchung fiel ein Säugling auf, bei dem Phenobarbital (44,9mg/l), Phenytoin (14,45mg/l), Thiopental (3,9mg/l) und Diazepam (0,225mg/l) nachweisbar war. Bei diesem Kind war jedoch eine intensivmedizinische Behandlung durchgeführt worden. Es kam nach primär erfolgreicher Reanimation zu kaum zu durchbrechenden generalisierten Krampfanfällen und Schnappatmung, daher erfolgte die Verabreichung hoher Dosen Luminal, Fentanyl, Diazepam, Phenytoin (Dauerinfusion), Thiopental (Dauerinfusion), Pankuronium, Suprarenin und Dopamin, womit sich die nachgewiesenen hohen Pharmakakonzentrationen erklären lassen.

Bei beiden Fällen mit positivem Morphinnachweis schlossen sich weitere polizeiliche Ermittlungen an, inklusive einer Untersuchung der Mutter, jedoch ohne greifbares Ergebnis. Die Konzentrationen müssen dabei jeweils als nicht todesursächlich angesehen werden. Im Übrigen ist grundsätzlich einzurechnen, daß negative chemischtoxikologische Untersuchungsergebnisse nur bezüglich der untersuchten Giftstoffe Gültigkeit besitzen.

Am 53 Blutproben wurden Bestimmungen der Blutalkoholkonzentration und Begleitstoffanalysen durchgeführt. Durch die Bestimmung der Blutalkoholkonzentration (BAK) ist ein Rückschluß auf die konsumierte Ethanolmenge möglich, durch eine Begleitstoffanalyse lassen sich zusätzlich Hinweise auf die Art des konsumierten Getränks gewinnen, wenn man das für das jeweilige Getränk typische Begleitstoffspektrum kennt [Wittmann 1996]. 
In neun Fällen war der Serumethanolspiegel erhöht, das heißt er lag über den in der Literatur zu findenden endogenen/ physiologischen Ethanolspiegeln [Gilg in Soyka 1995]. In einem Fall lag er sogar bei fast 0,4 \%o. Ethanol wird in der Regel oral aufgenommen. Die Resorption erfolgt passiv per diffusionem und beginnt theoretisch bereits in der Mundhöhle, was jedoch für die Praxis aufgrund der geringen resorbierten Menge keine Bedeutung hat. Die relevante Resorption findet zu 10-20\% im Magen und zu 80-90\% im Dünndarm, besonders im Duodenum, statt. Ethanol verteilt sich zu etwa 96\% im Körperwasser und zu 4\% im Fettgewebe. Der Verteilungsfaktor -bezogen auf das Körpergewicht- beträgt für Männer 0,7, für Frauen 0,6. Eliminiert wird Ethanol nur zu max. 5\% über Schweiß, Atmung und Urin. Der Rest wird, großenteils in der Leber, enzymatisch mittels ADH (Alkoholdehydrogenase), MEOS (mikrosomales ethanoloxidierendes System) und Katalase zu CO2 und $\mathrm{H} 2 \mathrm{O}$ abgebaut. Pro Stunde werden durchschnittlich 0,15\%o eliminiert [Gilg in Soyka 1995].

$\mathrm{Zu}$ diskutieren ist die Ursache für die gefundenen erhöhten Spiegel bei Säuglingen. In Frage kommt unter anderem eine endogene Bildung, eventuell alleine durch postmortale Veränderungen/ Fäulnis/ Autolyse oder durch Gärungsprozesse, möglicherweise aus Glukose. Immer in Betracht gezogen werden muß natürlich auch eine exogene Zufuhr durch die Eltern oder andere Personen, sei es akzidentell oder um das Kind ruhig zu stellen.

Um zu überprüfen, ob nicht möglicherweise auch eine Ethanolzufuhr mit der Babynahrung möglich ist, haben wir eine Begleitstoffanalyse an elf verschiedenen Babynahrungsprodukten (Obstbreie und -säfte) der Firmen Hipp und Alete durchgeführt. Es ergab sich hier ein Ethanolgehalt zwischen 0,04 und 0,29 g pro Liter Saft bzw. pro Kilogramm Brei. Pro einzelnem Babynahrungsgläschen mit 190g Inhalt wurden bis $\mathrm{zu} 0,08 \mathrm{~g}$ Ethanol nachgewiesen. Nimmt man beispielsweise ein sechs Kilogramm schweres Mädchen als Maßstab heran, so müßte dieses mindestens zehn von diesen Gläschen zu sich nehmen, um -rein rechnerisch und theoretisch- einen Serumethanolspiegel von 0,2\%o zu erreichen. $\mathrm{Zu}$ berücksichtigen ist aber auch noch der gastrische, und besonders der hepatische First-Pass-Effekt, der solche geringen Alkoholmengen sofort eliminieren müßte. In der Literatur finden sich nur wenige Angaben über Alkoholabbauwerte im Säuglingsalter, aber es gibt Hinweise auf eine beim Säugling noch nicht voll ausgebildete ADH-Aktivität. Stündliche 
Alkoholabbauwerte wie bei einem Erwachsenen werden scheinbar erst ab dem fünften Lebensjahr erreicht [Gilg in Soyka 1995].

Acetaldehyd entsteht im Carbohydrat- und Aminosäurestoffwechsel durch die Reduktion aus Ethanol mittels $\mathrm{ADH}$. Um zuverlässig den Acetaldehydspiegel bestimmen zu können, wäre eine sofortige Kühlung der frisch entnommenen Blutprobe und eine chemische Blockade der Enzymaktivität der Erythrozyten erforderlich. Bei den im Rahmen der Obduktion entnommenen Blutproben ist davon auszugehen, daß es durch Freisetzung von hämoglobin- bzw. erythrozytär gebundenem Acetaldehyd zu einer Erhöhung des Acetaldehydspiegels kommt. Auch bei hämolysiertem Blut sind erhöhte Spiegel meßbar [Gilg in Soyka 1995]. Bei den von uns untersuchten Kindern ergab sich allerdings kein Zusammenhang zwischen dem Serum-Acetaldehydspiegeln und der Leichenliegezeit bis zur Blutentnahme oder dem Serumethanolspiegel.

Der Methanolspiegel war in 11 Fällen auf über $2 \mathrm{mg} / \mathrm{l}$ erhöht. Es fiel auf, daß nur Kinder ab dem vierten Lebensmonat davon betroffen waren. Methanol entsteht im Wesentlichen nicht durch alkoholische Gärung, sondern wird aus Methylesterseitenketten des Pektins in pflanzlichen Produkten freigesetzt. Pektine sind mit Methanol veresterte Polymere der D-Galakturonsäure und in fast allen Pflanzen in unterschiedlicher Konzentration enthalten. Methanol ist ein in fast allen alkoholischen Getränken vorkommender Alkohol. Der Methanolgehalt allerdings variiert stark: Bier enthält wenige mg Methanol pro Liter, Wein zwischen 30 und $100 \mathrm{mg} / \mathrm{l}$, Obstbranntwein hingegen kann bis $\mathrm{zu} 6000 \mathrm{mg} / \mathrm{l}$ Methanol enthalten. Die Grundnahrungsmittel weisen keinen relevanten Methanolgehalt auf [Gilg in Soyka 1995]. In Untersuchungen von Gilg [1992] zeigte sich allerdings, daß die Aufnahme erheblicher Mengen von Obst oder Obstsäften (z.B. 13 Bananen oder 9 Äpfel) akut oder bei Vegetariern auch ohne Ethanolaufnahme zu Methanolspiegeln von bis $\mathrm{zu} 9 \mathrm{mg} / \mathrm{l}$ führen kann. Unter zusätzlicher Ethanolbelastung wurden Spiegel von bis zu fast 24 mg/l gemessen. Auch endogen wird Methanol gebildet: beispielsweise aus SAdenosylmethionin als Methylgruppendonator in der Hypophyse oder bei der Reduktion von endogen gebildetem Formaldehyd [Gilg in Soyka 1995]. Der durchschnittliche endogene Methanolspiegel liegt bei $0,95 \pm 0,45 \mathrm{mg} / 1$ [Gilg 1992]. Methanolspiegel über $10 \mathrm{mg} / \mathrm{l}$ im Serum ohne zuvor erfolgte Aufnahme methanolreicher Getränke werden in der Literatur als Hinweis auf Alkoholmißbrauch 
oder -abhängigkeit gewertet. Aufgrund guter Wasserlöslichkeit wird Methanol ebenso schnell resorbiert wie Ethanol, der Verteilungsfaktor liegt bei etwa 0,7. Die Elimination erfolgt wie beim Ethanol zu 90-95\% hepatisch-enzymatisch mittels ADH, MEOS und Katalase. Der Methanolabbau wird durch Ethanol kompetitiv gehemmt infolge einer 10bis 30-fach höheren Affinität der $\mathrm{ADH}$ für Ethanol: ab einer BAK von etwa 0,2 bis 0,5\% kann kein Methanol mehr abgebaut werden. Der Methanolspiegel ist nicht relevant von Lagerungs- und Fäulnisveränderungen betroffen [Gilg in Soyka 1995].

Bezüglich der auffälligen Altersverteilung der erhöhten Methanolspiegel (alle Kinder älter als vier Monate) findet sich eine mögliche Erklärung: In Ernährungsempfehlungen verschiedenen Hersteller von Babynahrung läßt sich nachlesen, daß ab dem vollendeten vierten Lebensmonat die Kinder erstmals und anhaltend mit obsthaltigen Nahrungsmitteln gefüttert werden. Zur Klärung der Frage, wieviel Methanol diese Babynahrung enthält, haben wir an den elf oben erwähnten Babynahrungsprodukten der Firmen Hipp und Alete eine Methanolbestimmung durchgeführt. Erwartungsgemäß ergab sich ein Methanolgehalt von 27 bis zu 144 mg pro Liter Obstsaft oder Kilogramm Obstbrei. Pro Fläschchen waren bis zu $27 \mathrm{mg}$ Methanol nachweisbar. Durch den Verzehr eines dieser Gläschen würde ein sechs Kilogramm schweres Kind rein theoretisch einen Serummethanolspiegel von $0,7 \mathrm{mg} / \mathrm{l}$ erreichen. Um einen Serummethanolspiegel von $3 \mathrm{mg} / 1$-nach Abzug des rein endogenen Methanols- zu erreichen, müßte ein Kind nur etwa vier dieser Gläschen zu sich nehmen. Die erhöhten Methanolspiegel lassen sich daher am ehesten auf eine Nahrungsumstellung auf obsthaltige Nahrung ab dem vierten Lebensmonat zurückführen.

Aceton entsteht bei sekundären Stoffwechselveränderungen z. B. bei diabetischer Ketoazidose, Hungerketose oder Unterkühlung. Relevante Acetonkonzentrationen kommen in alkoholischen Getränken nicht vor. Isopropanolspiegel liegen zwischen 0,02 und $0,1 \mathrm{mg} / 1 \mathrm{im}$ physiologischen Bereich, in erster Linie als Folge der Reduktion des biochemischen Reaktionspartners Aceton [Gilg in Soyka 1995]. In unseren Untersuchungen ließ sich eine angedeutet Korrelation zwischen beiden Reaktionspartnern nachweisen (siehe Abb. 16). Nach mehrstündigem Fasten kann die Isopropanolkonzentration im Serum auf über $6 \mathrm{mg} / \mathrm{l}$ ansteigen. Ebenso kommt es zu einem resorptiven Anstieg des Isopropanol-Serumspiegels nach präoperativer großflächiger Hautdesinfektionen mit isopropanolhaltigen Antiseptika, was bei den von 
uns untersuchten Kindern jedoch nicht von Relevanz war [Peschel 1992]. Am ehesten ließen sich die in einzelnen Fällen erhöhten Acetonspiegel wohl durch eine mögliche, dem Tod vorausgehende Nahrungsreduktion oder -karenz erklären.

Möglicherweise spielt die bisher noch nicht ausreichend untersuchte Unreife der alkoholabbauenden Enzymsysteme beim Säugling eine entscheidende Rolle.

\subsection{Betrachtung der Non-SIDS-Fälle: Gibt es überhaupt ein SIDS?}

In Kapitel 4.8. wurden die Besonderheiten der Non-SIDS-Fälle zusammenfassend dargestellt.

Von immenser Bedeutung ist die Beobachtung, daß in 95\% (!) der obduzierten/ untersuchten Fälle Hinweise auf (leichte oder schwere) Erkrankungen oder vereinzelt auch Gewalttaten vorlagen, und damit die Ausschlußdiagnose „SIDS“ nicht mehr gestellt werden kann (auf die Problematik dieser Definition und deren Verwendung wird in Kapitel 6.7. detaillierter eingegangen). Somit haben wir kaum typische SIDS-Fälle finden können. Hierfür kommen zwei mögliche Ursachen in Frage:

Entweder ist die derzeit gültige Diagnoserichtlinie für das SIDS mangelhaft, muss überarbeitet werden und unseren -sicher auch in vergleichbar umfangreichen Untersuchungen anderer Institute reproduzierbaren- Untersuchungsergebnissen und damit dem aktuellen Stand der Wissenschaft angeglichen werden (Kapitel 6.7.).

Oder aber man verbindet diese SIDS-Definition und unsere Untersuchungsergebnisse, dann bleibt nur ein möglicher Rückschluss: Es gibt keinen Plötzlichen Säuglingstod!

Eine ergänzende Begründung könnte lauten, daß wir bei den 5\% der Todesfälle, bei denen wir keine pathologischen Befunde aufdecken konnten, derzeit noch nicht nach den richtigen Viren gesucht, die richtigen Organe mikroskopiert oder andere Untersuchungen (z.B. immunologischer oder genetischer Art) unterlassen haben, die die Todesursache aufgedeckt hätten. Es könnte also mangels diagnostischer Möglichkeiten, möglicherweise auch einfach finanzieller (staatsanwaltschaftlich angeordnete Obduktionen) oder zeitlicher Eingeschränktheit zuzuschreiben sein, daß bei diesen Kindern keine Todesursache gefunden wurde, obwohl eine solche vorhanden sein muß. 
Die Tatsache, daß wir durch gegenüber der Routine massiv ausgeweiteter Diagnostik bereits bei 95\% der Kinder Auffälligkeiten nachweisen konnten, legt die Annahme nahe, daß mit noch größerem Aufwand auch bei den restlichen 5\% eine Ursache für den Tod nachweisbar wäre. Diese Nachweisbarkeit übersteigt jedoch möglicherweise derzeitige diagnostische Möglichkeiten.

Verfolgt man diesen durchaus nicht unwahrscheinlich erscheinenden „Es-gibt-keinSIDS-Gedanken“" weiter, so ergäben sich vielfältige notwendige Konsequenzen und völlig neue Perspektiven, die einerseits für die betroffenen Eltern, andererseits auch für die weitere SIDS-Ursachenforschung von größter Bedeutung wären.

Für die Eltern wäre Folgendes von großer Relevanz:

- Das Kind wäre an einer greifbaren, definierten Erkrankungen verstorben, nicht aufgrund irgendeines, manchmal fast schon mythisch anmutenden, ungeklärten, unabwendbaren und unvorhersehbaren Phänomens. Dies könnte einerseits eine Erleichterung für die betroffenen Eltern darstellen: Man wüsste nun, daß das Kind krank war und an den Folgen einer Erkrankung verstorben ist. Andererseits könnte sich eine erhöhte Belastung aufgrund von (Selbst-)Vorwürfen für die Eltern einstellen: Warum haben wir die Erkrankung nicht bemerkt? Warum hat unser Kinderarzt sie nicht bemerkt? Warum wurde dem Kind nicht rechtzeitig geholfen?

- Es gäbe keine familiäre Häufung des Plötzlichen Säuglingstodes, denn es könnte sich bei mehrfachen Todesfällen höchstens um zufällig in einer Familie gehäuft auftretende anderweitige Todesfälle handeln. Diese Annahme sollte für betroffene Eltern eigentlich eine Erleichterung darstellen, die Wahrscheinlichkeit für ein zweites SIDS-Kind wäre als extrem gering darzustellen, allerdings mit der Ausnahme angeborener Stoffwechselerkrankungen, die aber vielleicht bei rechtzeitiger Diagnose nicht zum Tode führen würden. Die weitere Familienplanung könnte wesentlich entspannter ablaufen, als sie es momentan in den meisten Fällen vermutlich tut. Die Suche nach Todesursachen wie Tötungen durch das Münchhausen-Syndrom-by-Proxy allerdings bekämen in Familien mit mehr als einem "SIDS-Fall" eine ganz andere, wesentlich größere Bedeutung.

- Es wäre für die Eltern eine Erleichterung sicher zu wissen, daß das Kind nicht, wie oftmals angenommen und durch Vorwürfe Dritter bekundet, im Kissen erstickt oder 
durch „unintentional overlaying“ getötet wurde, sondern dass es sich um einen natürlichen, krankheitsbedingten Tod handelte.

Für die weitere SIDS-Forschung ergäben sich ebenfalls vielfältige neue Aufgaben:

- Die Suche nach der Ursache des Todeseintritts

- Etablierung von Vorsorgeuntersuchungen

- Entwicklung von Screeningverfahren für gefährdete Kinder und von Testverfahren, um die Bedrohung einer eingetreten Infektion einschätzen zu können

- Schulung der Eltern dieser bedrohten Kinder, dazu jedoch erst einmal Entwicklung von Eingriffs-/Therapiemöglichkeiten

- Würdigung des SIDS durch die Pharmaindustrie und Entwicklung von präventiv und therapeutisch anwendbaren Medikamenten und Überprüfung von Impfmöglichkeiten. Es erscheint im Übrigen bemerkenswert, daß sich bisher die Pharmaindustrie in keiner Weise des SIDS angenommen hat, und sei es nur zum Verkauf immunstimulierender Medikamente, die zur Prävention angeboten werden könnten.

Es gibt somit insgesamt eine Vielzahl von Beobachtungen und Überlegungen, die dafür sprechen, daß der sogenannte „Plötzliche Säuglingstod“ nicht so plötzlich und unerwartet ist, wie bisher angenommen.

\subsection{Diskussion der Totenscheinanalyse}

Die durchgeführte Totenscheinanalyse läßt keinen Rückschluß auf die Obduktionsrate von Säuglingen zu, da wir feststellen mußten, daß uns von den Gesundheitsämtern leider teilweise nicht alle Todesbescheinigungen zugesandt wurden. Immer wieder kam es vor, daß wir von einem obduzierten Säugling keine Todesbescheinigung erhielten. Wir konnten die Diagnosen in diesen Bescheinigungen nur bei den 70 obduzierten Kindern verifizieren, von denen wir eine Todesbescheinigung erhalten haben.

Immerhin 14 dieser 70 Bescheinigungen waren so ausgefüllt, daß sie entweder gar keine Verdachtsdiagnose enthielten, nur ein Fragezeichen oder Formulierungen wie 
„unklar“ oder „nicht bekannt“" verwendet wurden. In einem Fall wurde eine reflektorische Apnoe diagnostiziert, deren Nachweis für den Leichenschauer wohl unmöglich sein dürfte. Eine andere Todesbescheinigung enthielt die (Verlegenheits-) Diagnose „Herzstillstand“ als Todesursache, diese Todesbescheinigung haben wir als fehlerhaft bewertet.

Bei vier Kindern wurde als Verdachtsdiagnose „Ersticken“ angegeben, aber nur in zwei Fällen wurde eine Obduktion angeordnet. In diesen Fällen konnte ein Plötzlicher Kindstod (Kategorie 2) bestätigt werden. Aus der Diagnose „Verdacht auf Ersticken“ kann man keine Rückschlüsse darauf ziehen, ob der leichenschauende Arzt vielleicht doch einen SIDS meinte, oder ob er tatsächlich einen Anhalt für einen nicht natürlichen Tod gehabt hat. Daß in drei Fällen der Verdacht auf Plötzlichen Kindstod und die „natürliche Todesart“ kombiniert wurden, ist nachvollziehbar, wenn man davon absieht, daß man ein SIDS nicht ohne Obduktion diagnostizieren kann. Interessant war, daß auch vielfach die Kombination „V.a. SIDS“ mit „nicht natürlicher Tod“ verwendet wurde.

Leider erhielten wir von den Gesundheitsämtern unter anderem von keinem der vier Tötungsdelikte eine Todesbescheinigung, so daß wir nicht überprüfen konnten, ob dem leichenschauenden Arzt hier ein Anhalt für einen nicht natürlichen Tod auffiel.

Insgesamt zeigt diese Totenscheinanalyse, daß vermutlich vielen Ärzten nicht bekannt ist, daß es sich bei dem SIDS um eine Ausschlußdiagnose nach blander Obduktion handelt. Nicht unproblematisch erscheint weiterhin, dass mehrfach ein Erstickungstod angenommen wurde, was für die betroffenen Eltern selbst, vorausgesetzt sie hätten nicht aktiv zum Tod des Kindes beigetragen, eine massive Schuldzuweisung bedeuten könnte. Es bereitete offensichtlich auch Schwierigkeiten, den Plötzlichen Säuglingstod als natürlichen oder nicht natürlichen Tod zu klassifizieren.

\subsection{Diskussion des Begriffs und der Definition des „SIDS“}

Die Bezeichnung „SIDS“ ist unter Medizinern, aber auch unter medizinischen Laien, fest etabliert und wird vermutlich oft unüberlegt verwendet. 
Letztlich ist die Verwendung des Begriffs „Sudden Infant Death Syndrome“ für den Plötzlichen Säuglingstod völlig unzutreffend schon deswegen, weil sich ein Syndrom definitionsgemäß aus mehreren Symptomen zusammensetzt. Beim Plötzlichen Säuglingstod handelt es sich aber lediglich um ein Symptom, nämlich daß das Kind plötzlich und unerwartet verstorben ist. Hier sei noch einmal auf die schon in der Einleitung zitierte Aussage Rognums verwiesen: „SIDS ist ein Syndrom, dessen erstes und einziges Symptom der Tod ist!“ [Rognum et al. 1995]. Von vielen Wissenschaftlern wird daher der Begriff „SID“, also nur „Sudden Infant Death“ ohne das „Syndrom“, verwendet.

Nach Abschluß unserer Untersuchungen handelt es sich jedoch weit eher bei den Kindern, die plötzlich und unerwartet versterben, um „SUD“-Fälle (= Sudden Unexplained Death) und natürlich um „SID“-Fälle (= Sudden Infant Death), denn nach Abschluss sämtlicher Untersuchungen werden viele dieser Kinder zu „SEID“- Fällen (=Sudden Explained Infant Death) geworden sein. Die Kinder, bei denen sich keine Todesursache ermitteln läßt, bleiben „SUD“-Fälle. Sie bleiben ebenfalls „SID“-Fälle. Es würde sich daher anbieten, diese Kinder als SUID-Fälle zu bezeichnen: Sudden Unexplained Infant Deaths (Abb. 24):

Abb. 24: Vorschlag für eine neue SIDS-Nomenklatur

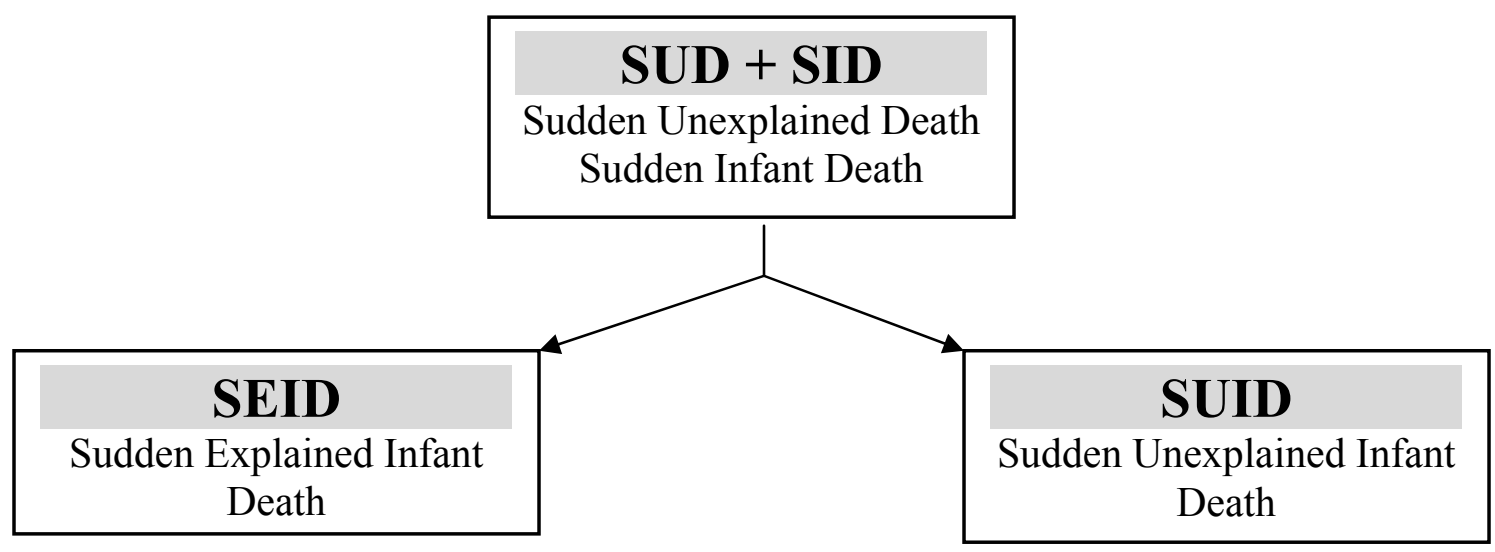

Auch die Definition des Plötzlichen Säuglingstodes bereitet Schwierigkeiten. Nach wie vor ist die 1970 von Beckwith aufgestellte die am weitesten verbreitete und akzeptierte Definition geblieben (siehe auch Kapitel 1.1.). 
Das erste Problem besteht darin, daß in dieser Definition keine Eingrenzung des Alters der verstorbenen Kinder erfolgt. Daher finden sich in der Literatur auch SUID-Studien [Riße 2001], die Kinder bis zum vollendeten zweiten Lebensjahr oder sogar noch ältere Kinder eingeschlossen haben. Ebenso finden sich auch viele Publikationen über plötzlich unerwartet verstorbene Kinder, die noch in der neonatalen Periode verstarben (erster bis siebenter Lebenstag). Im Allgemeinen ist jedoch die Meinung verbreitet, daß es sich bei SUID-Fällen nur um Kinder handeln sollte, die zwischen dem achten und 365. Lebenstag verstorben sind, um so beispielsweise direkte Folgeerkrankungen der Entbindung ausschließen zu können.

Weiter ist laut Definition unabdingbare Voraussetzung für die Diagnosestellung eine Autopsie, in der dann keine pathologischen Befunde gefunden werden, laut Aoki eine „negative Autopsie“ [Aoki 1994]. Wie aber den vorigen Kapiteln zu entnehmen ist, herrscht inzwischen der Meinung vor, dass zu einem Plötzlichen Säuglingstod sogar typischerweise leichte Anzeichen für (Atemwegs-)Infektionen gehören. Auch ist das Vorhandensein von leichten Fehlbildungen, auch kardiovaskulärer Art, kein Ausschlußkriterium für die Diagnose SUID.

In der Definition wird auch nicht der genaue Umfang der durchzuführenden Untersuchungen angegeben. Gehören histologische, bakteriologische, toxikologische, immunhistochemische oder gar molekulargenetische Untersuchungen zur Diagnosestellung dazu? Oder reicht es quasi aus, wenn die Todesursache nicht mit bloßem Auge erkennbar ist?

Es dürfte allerdings bereits ein Problem sein, innerhalb eines Landes eine einheitliche Strategie mit einem bestimmten Untersuchungsspektrum zur Diagnosestellung durchzusetzen. Selbst an Rechtsmedizinischen Instituten, die in Deutschland den Großteil der verstorbenen Säuglinge obduzieren, könnte eine korrekte Diagnosestellung schon daran scheitern, daß es sich meist um staatsanwaltschaftlich angeordnete Obduktionen handelt. Für die -kostenorientierte- Staatsanwaltschaft ist verständlicherweise nur von Belang, ob das Kind an einem natürlichen oder nicht natürlichen Tod verstorben ist, jedoch nicht, welches Bakterium genau nun das Kind infiziert hat oder wie ausgeprägt die histologischen Befunde sind.

Deshalb ist davon auszugehen, daß vermutlich auch der international veröffentlichten SUID-Literatur unterschiedliche diagnostische Methoden und durchgeführte 
Untersuchungen zugrunde liegen, und deshalb die dort erhobenen Daten, und vor allem Fallzahlen, nicht generell verglichen werden können.

Im Widerspruch zu allen Definitionen aber findet sich häufig schon in den Todesbescheinigungen der verstorbenen Kinder die Diagnose „SIDS“, obwohl diese Diagnose nur nach erfolgter Obduktion gestellt werden kann. Dabei ist es für den leichenschauenden Arzt meistens nicht möglich, ohne Obduktion beispielsweise eine Erstickung, ein Schütteltrauma, eine Intoxikation oder eine schwere Infektion festzustellen. 


\section{SCHLUßFOLGERUNGEN}

Aus den Ergebnissen dieser Arbeit ergeben sich zusammengefaßt folgende Schlußfolgerungen:

Die in dieser Studie gewonnen epidemiologischen Daten entsprechen im Allgemeinen den in der internationalen Literatur veröffentlichten. Für viele dieser so „SIDStypischen“" Erscheinungen, wie beispielsweise Auffindung in Bauchlage oder ,junge Mütter", gibt es diverse Theorien und Erklärungsversuche, aber für keine dieser Beobachtungen gibt es eine eindeutige Erklärung.

Die makroskopischen, in der Obduktion gewonnen Erkenntnisse, entsprechen nicht den in der histologischen Untersuchung aufgedeckten pathologischen Veränderungen. Es kann nur eine Gesamtschau der makroskopischen und mikroskopischen Befunde zur Diagnose und damit der Todesursache führen, wobei eine umfassende histologische Untersuchung, wie in dieser Studie durchgeführt, von großer Bedeutung ist.

Die Befunde der bakteriologischen und virologischen Untersuchungen waren oft völlig überraschend, bezogen auf den Obduktionsbefund. Diese Untersuchungen sind ein unabdingbares diagnostisches Hilfsmittel bei der abschließenden Beurteilung des Falls, wobei noch mehr Gewicht auf die Virologie gelegt werden sollte.

Eine -sehr kostenintensive- toxikologische Untersuchung müsste eigentlich sicherheitshalber in jedem Fall erfolgen, zumindest aber, wenn ein Verdachtsmoment vorliegt oder anamnestisch Angaben für eine Medikamenteneinnahme, vielleicht auch eine medikamentöse Therapie der Mutter, vorliegen.

Durch die Begleitstoffanalysen ergab sich kein Hinweis auf eine pathognomonische Bedeutung erhöhter Spiegel von Ethanol, Methanol oder Aceton für den Plötzlichen Säuglingstod. Ebenso ergaben sich keine Hinweise auf eine andere, regelmäßig beim SID vorkommende, möglicherweise bisher unbekannte flüchtige Substanz. Es sollte daher in den einzelnen Fällen mit erhöhten Spiegeln immer auch eine exogene Zufuhr, sei es akzidentell oder beabsichtigt, durch die Eltern oder andere in Betracht gezogen 
werden, und gegebenenfalls gezielt über die ermittelnden Kriminalpolizeibeamten in dieser Richtung gefahndet werden.

Es besteht dringender Bedarf an der Schaffung einer aktualisierten und dem derzeitigen Stand der Wissenschaft angepaßten Definition für den Plötzlichen Säuglingstod.

Es ist notwendig, ein national, oder besser noch international, standardisiertes Vorgehen bei der Untersuchung von Plötzlichen Säuglingstodsfällen festzulegen: Kriterien bei der Anamneseerhebung und der Obduktion und Festlegung der durchzuführenden Zusatzuntersuchungen (Histologie, Bakteriologie, Toxikologie etc.) mit den entsprechenden Asservaten. Ohne Standard ist ein Vergleich der internationalen Daten, und damit vielleicht einmal die Erforschung der Ursache(n) des Plötzlichen Säuglingstodes, deutlich erschwert oder gar nicht möglich, denn unsere Untersuchungen haben gezeigt: je mehr Untersuchungsmethoden angewandt werden, desto eher besteht die Chance, die Todesursache klären zu können.

Ebenso besteht Schulungsbedarf vor allem für Leichenschauen durchführende Ärzte bezüglich der korrekten Verdachtsdiagnose in der Todesbescheinigung und der Notwendigkeit der Durchführung einer Obduktion zur Diagnosestellung und Klärung der Todesursache, denn dies ist ohne Obduktion (und zahlreiche Zusatzuntersuchungen) nicht ansatzweise möglich. Ebenso muss vermittelt werden, dass nur die Bescheinigung der ungeklärten Todesart zur gerichtlich angeordneten Sektion führen kann.

Wichtigstes Ergebnis dieser Arbeit ist der Schluss, dass der Plötzliche Säuglingstod in der bisher diskutierten Form vermutlich nicht existiert.

Wahrscheinlich handelt es sich beim „SUID“ um den Tod eines Säuglings, der in einer (möglicherweise viralen) Infektion seinen Anfang nimmt. Durch bisher nicht bekannte Einflußfaktoren oder abnorme Reaktionen (Genetische Disposition? Pathologische Immunreaktion? Besondere Keim-Pathogenität?) kommt es in letzter Konsequenz zum Todeseintritt. Aufgrund eingeschränkter methodischer, wissenschaftlicher und nicht zuletzt finanzieller Möglichkeiten ist man bisher nicht in der Lage, dem/den Auslöser(n) des SUID auf die Spur zu kommen. Der Aufdeckung dieser Faktoren kommt in der weiteren SUID-Forschung die größte Bedeutung zu. 


\section{ZUSAMMENFASSUNG}

Im Rahmen der multizentrischen BMBF-Studie „Plötzlicher Säuglingstod“(Leitung Westfälische Wilhelms-Universität Münster) wurden in den Jahren 1999 bis 2001 am Münchener Institut für Rechtsmedizin 93 verstorbene Säuglinge staatsanwaltschaftlich obduziert. 80 der Kinder konnten in die Studie eingeschlossen werden. Von allen Säuglingen wurden epidemiologische Daten erhoben und eine rechtsmedizinische Obduktion wurde durchgeführt. Weiter wurden eine bakteriologische, virologische und toxikologische Untersuchung sowie eine Serumethanolbestimmung und eine Begleitstoffanalyse durchgeführt. Aus im Rahmen der Obduktion entnommenen Gewebsproben wurden umfangreiche histologische Untersuchungen anhand von 80 Präparaten aus 39 verschiedenen Organen bzw. Körperregionen durchgeführt. Nach Abschluß sämtlicher Untersuchungen wurden die Kinder im Rahmen einer „Fallbesprechung“ einer von der Studienzentrale vorgegebene Kategorie von 1 bis 4 zugeordnet, wobei die Kategorien eins und zwei die sog. SIDS (=Sudden Infant Death Syndrome)-Fälle umfaßten, die Kategorien drei und vier die Kinder mit aufgedeckter wahrscheinlicher oder sicherer anderer Todesursache. Die durchgeführten Untersuchungen erbrachten folgende Ergebnisse:

Die epidemiologischen Daten wie Alter, Geschlecht, Auffindesituation etc. der verstorbenen Säugling standen weitestgehend mit den in der internationalen Literatur publizierten Daten in Einklang, wobei es nach wie vor für diese „SIDS-typischen“ Verteilungsmuster keine ausreichende Erklärung gibt.

Die histologischen Untersuchungen erwiesen sich als sehr bedeutend, was die Feststellung der Todesursache anging. Es konnten bei einem Viertel der Kinder leichte bis schwere Pneumonien nachgewiesen werden, die alle makroskopisch allenfalls vermutet worden waren, und zum Teil durchaus als todesursächlich angesehen werden können. Es ließen sich auch andere bedeutende Befunde wie Myokardinfarkte, Williams-Beuren-Syndrome, Myokarditiden etc. nachweisen, die ohne umfangreiche histologische Untersuchungen unbemerkt geblieben wären. Insgesamt konnten bei über $90 \%$ der Kinder pathologische histologische Befunde erhoben werden.

Durch die mikrobiologischen Untersuchungen anhand verschiedener Untersuchungsmaterialien im Max-von-Pettenkofer-Institut in München wurden bei 32\% der Kinder pathogene Keime nachgewiesen (am häufigsten Staphylokokkus aureus), die in mehreren Fällen sogar Hinweise auf das Vorliegen einer Sepsis gaben. 
Virologische Untersuchungen wurden in Erlangen durchgeführt, bislang nur an Stuhlproben und Trachealabstrichen. Bei 23\% der Kinder wurden pathogene Viren nachgewiesen, am häufigsten Zytomegalieviren bei $15 \%$ der Kinder.

Die toxikologische Untersuchung ergab, außer zwei positiven Morphinnachweisen ungeklärter Ursache, keine relevanten Hinweise auf Intoxikationen.

Im Rahmen der Serumethanolbestimmung und Begleitstoffanalyse fielen bei neun Kindern Ethanolspiegel von mehr als 0,1 Promille auf, so wie in elf Fällen unphysiologisch hohe Serummethanolspiegel von mehr als $2 \mathrm{mg} / \mathrm{l}$. Eine Erklärung für erhöhte Ethanolspiegel konnten wir nicht finden. Die erhöhten Methanolspiegel sind am ehesten, da sie ausschließlich Kinder mit einem Alter von mehr als vier Monaten betrafen, auf eine veränderte Ernährung des Kindes mit obsthaltigen Produkten zurückzuführen. Zur Verifizierung dieser Annahme haben wir auch eine Ethanol- und Methanolbestimmung an elf Babynahrungsprodukten durchgeführt, die unsere Annahme teilweise bestätigten, da die Babynahrungsprodukte verschiedener Firmen sowohl Ethanol als auch Methanol in geringen Mengen enthielten.

Nach Abschluß aller dieser Untersuchungen wurden die Kinder kategorisiert. Es konnten nur 5\% der Kinder der Kategorie 1 (keinerlei pathologische Befunde in irgendeiner Untersuchung) zugeordnet werden, also als eigentliche SIDS-Fälle per definitionem eingestuft werden. Der Großteil der Kinder (60\%) wurde der Kategorie 2 zugeordnet (leichte pathologische Veränderungen oder Auffälligkeiten, die den Tod jedoch nicht erklären können). 13\% wurden in Kategorie 3 eingeordnet (pathologische Veränderungen, die durchaus mit dem Todeseintritt in Verbindung stehen können) und in $22 \%$ der Fälle konnten wir eine sichere Todesursache nachweisen (Kategorie 4).

Durch die Obduktion dieser Kinder, die alle mit der Verdachtsdiagnose „Plötzlicher Säuglingstod“" obduziert wurden, konnten auch vier Tötungsdelikte aufgedeckt werden.

Diese Ergebnisse lassen deutlich werden, dass es den plötzlichen Säuglingstod, so wie er im Allgemeinen bekannt ist, nicht gibt. Bei sorgfältiger, umfangreicher Untersuchung kann man vielfach Hinweise auf die Todesursache erhalten, die ohne Obduktion und vor allem histologische und bakteriologische Untersuchungen dem Nachweis entgangen wären.

Ohne unsere Untersuchungen wäre fast die Hälfte der in dieser Studie untersuchten Kinder, zum Teil mit gravierenden Konsequenzen (weitere Familienplanung, kriminelle Delikte, weitere Infektionsfälle in der Familie etc.), als SIDS-Fälle fehldiagnostiziert worden. 


\section{LITERATURVERZEICHNIS}

1. Adelson L, Kinney ER (1956) Sudden and unexpected death in infancy and childhood. Pediatrics 17:663-97

2. Alessandri LM, Read AW, Burton PR, Stanley FJ (1996) Sudden infant death syndrome in Australian aboriginal and non-aboriginal infants: an analytic comparison. Paediatr Perinat Epidemiol 10(3):309-18

3. Althoff H (1986) Methodik und Ergebnisse postmortaler Nasen-RachenUntersuchungen bei Todesfällen im Säuglingsalter, speziell bei plötzlichen Kindstodesfällen. Pathologe 7(4):207-12

4. Aoki Y (1994) Histopathological findings of the lung and trachea in sudden infant death syndrome: Review of 105 cases autopsied at Dade Country Medical Examminer Department. Jpn J Legal Medicine 48(3):141-9

5. Arntzen A, Moum T, Magnus P, Bakketeig LS (1995) Is the higher postneonatal mortality in lower social status groups due to SIDS? Acta Paediatr 84(2):188-92

6. Bajanowski T, Rossi L, Biondo B, Ortmann C, Haverkamp W, Wedekind H, Jorch G, Brinkmann B (2001) Prolonged QT interval and sudden infant death report of two cases. Forensic Sci Int 115(1-2):147-53

7. Beal SM (1983) Some epidemiological factors about sudden infant death syndrome (SIDS) in South Australia. In: Tildon JT, Roeder LM, Steinschneider A (Hrsg.): Sudden Infant death Syndrome, Academic Press, New York London Paris

8. Beal S (1989) Sudden infant death syndrome in twins. Pediatrics 84(6):1038-44

9. Beckwith JB (1970) Discussion of the terminology and definition of the sudden infant death syndrome. In: Bergmann AB, Beckwith JB, Ray CC (Hrsg.): Sudden infant death syndrome. Proceedings of the Second International Conference on the causes of the sudden death in infants, University of Washington Press, Seattle London. S. 83 - 139

10. Beckwith JB (1988) Intrathoracic petechial hemorrhages: a clue to the mechanism of death in sudden infant death syndrome? Ann N Y Acad Sci $533: 37-47$

11. Beckwith JB (1993) A proposed new definition of sudden infant death syndrome. In: Walker AM, McGillen C (Hrsg.): Second SIDS International Conference 1992, Perinatology Press, Ithaca New York USA. S. $421-424$

12. Bell S, Crawley BA, Oppenheim BA, Drucker DB, Morris JA (1996) Sleeping position and upper airways bacterial flora: relevance to cot death. J Clin Pathol 49:170-2 
13. Boeck P (Hrsg.), Denk H, Künzle H, Plenk jr. H, Rüschoff J, Sellner W (1989) Romeis- Mikroskopische Technik, 17. Aufl., Urban und Schwarzenberg München Wien Baltimore

14. Bonser RS, Knight BH, West RR (1978) Sudden infant death syndrome in Cardiff, association with epidemic influenza and with temperature - 1955 1974. Int J Epidemiol 7:335-8

15. Borhani NO, Rooney PA, Kraus JF (1973) Postneonatal sudden unexplained death in a califormia community. Calif Med 118:12-6

16. Bouden A, Krebs MO, Loo H, Olie JP (1996) Munchhausen syndrome by proxy: a challenge for medicine. Press med 25(12):567-9

17. Byard RW, Krous HF (1999) Suffocation, shaking or sudden infant death syndrome: Can we tell the difference? J Pediatr Child Health 35(5):32-3

18. Carpenter RG (1983) The Search for Practical Predictors of Risc. In: Tildon JT, Roeder LM, Steinschneider A (Hrsg.): Sudden Infant death Syndrome, Academic Press New York London Paris

19. Côté A, Gerez T, Brouilette RT, Laplant S (2000) Circumstances Leading to a Change to Prone Sleeping in Sudden Infant Death Syndrome Victims. Pediatrics 106(6).E86

20. Cremer U, Althoff $\mathrm{H}$ (1991) Nachweis und Inzidenz von Zytomegalievirusinfektionen bei plötzlichen Kindstodesfällen (SIDS). Rechtsmed 1:25-8

21. Daltveit AK, Irgens LM, Oyen N, Skjaerven R, Markestad T, Alm B, Wennergren G, Norvenius G, Helweg-Larsen K (1998) Sociodemographic risk factors for sudden infant death syndrome: associations with other risk factors. The Nordic Epidemiological Study. Acta Paediatr (1998) 87(3):284-90

22. Douglas AS, Helms PJ, Jolliffe IT (1998) Seasonality of sudden infant death syndrome (SIDS) by age at death. Acta Paediatr 87(10):1033-8

23. Einspieler C (2000) Potentielle Risikofaktoren aus der Umgebung des Säuglings. In: Kurz R, Kenner Th, Poets Ch (Hrsg.): Der plötzliche Säuglingstod. Ein Ratgeber für Ärzte und Betroffene. Springer Verlag Wien New York. S. 165-166

24. Emery JL (1983) A way looking at the causes of Crib death. In: Tildon JT, Roeder LM, Steinschneider A (Hrsg.): Sudden Infant Death Syndrome, Academic Press New York London Paris

25. Fearn SW (1834) Sudden and unexplained death of children. Lancet 1:246 
26. Gesellschaft für wissenschaftliche Datenverarbeitung mbH (2002) Zytomegalie. Im Internet unter: www.gwdg.de/ khoeche/Zytomegalie.html

27. Gilg Th (1992) Methanol: Stoffwechsel bei kurzzeitiger und chronischer Alkoholaufnahme und Stellenwert als biochemischer Alkoholismusmarker. Habilitationsschrift, Institut für Rechtsmedizin der Universität München

28. Gilg Th (1995) Alkohol (Ethanol): Pharmakologie, BAK_Berechnung und forensische Begutachtung IN: Soyka M (1995) Die Alkoholkrankheit Diagnose und Therapie. Chapman \& Hall London, Glasgow, Weinheim, New York, Tokyo, Melbourne, Madras

29. Goldberg J, Hornung R, Yamashita T, Wehrmacher W (1986) Age at death and risk factors in sudden infant death syndrome. Aust Paediatr J 22 Suppl 1:21-28

30. Guntheroth WG, Lohmann R, Spiers PS (1990) Risk of sudden infant death syndrome in subsequent sibbling. J Pediatr 116:520-4

31. Guntheroth WG (1995) Crib death: The sudden infant death syndrome. Third Edition, Library of Congress Cataloging-in-Publication Data, Futura Publishing Company, Inc. Armonk New York

32. Ho GY, Windsor HM, Snowball B, Marshall (2001) Helicobacter pylori is not the cause of sudden infant death syndrome. Am J Gastroenterol 96(12):3288-94

33. Hoffmann HJ, Damus K, Hillmann L, Krongrad E (1988) Risk factors for SIDS. Results of the National Institute of Child Health and Human Development Cooperative Epidemiological Study. Ann N Y Acad Sci 533:13-30

34. Irwin KL, Mannino S, Daling J (1992) Sudden infant death syndrome in Washington State: Why are Native American infants at greater risk than white infants? J Pediatr 121(2):242-7

35. Jorch G, Schellscheidt J, Bajanowski T, Ott A, Findeisen M, Brinkmann B (1995): Westfälische Kindstodstudie - Prä-/ perinatale Risiken. In: Althoff H (Hrsg.): Rechtsmedizin von A - Z, Von der Arztethik bis zur zweifelhaften Vaterschaft, Kongressbericht der 74. Jahrestagung der Deutschen Gesellschaft für Rechtsmedizin, Verlag Murken-Altrogge Herzogenrath. S. 162

36. Jorch G (2000) SIDS-Mortalität im deutschsprachigen Raum und weltweit. In: Kurz R, Kenner Th, Poets Ch (Hrsg.): Der plötzliche Säuglingstod. Ein Ratgeber für Ärzte und Betroffene, Springer Verlag Wien New York. S.21 - 38

37. Kariks J (1988) Cardiac Lesions in Sudden Infant Death syndrome. Forensic Sci Int 39(3):211-25 
38. Kenner T, Kerbl R (2000) Mögliche Ursachen für den plötzlichen Säuglingstod. In: Kurz R., Kenner Th, Poets Ch (Hrsg.): Der Plötzliche Säuglingstod. Ein Ratgeber für Ärzte und Betroffene, Springer Verlag Wien New York. S. 133 144

39. Kerr JR, Al-Khattaf A, Barson AJ, Burnie JP (2000) An association between the sudden infant death syndrome (SIDS) and Helicobacter pylori infection. Arch Dis Child 83(5):429-34

40. Kleemann WJ (1996) Der plötzliche Kindstod - Epidemiologie, Morphologie, Pathogenese. Habilitationsschrift zur Erlangung der venia legendi für das Fach Rechtsmedizin aus dem Institut für Rechtsmedizin der Medizinischen Hochschule Hannover, Direktor Prof. Dr. med. H.D. Tröger

41. Kleemann WJ, Urban R, Eidam J, Wiechmann B, Tröger HD (1991) Die Auffindesituation beim plötzlichen Kindstod. Rechtsmed 1:147-51

42. Kleemann WJ, Schlaud M (1995a) Bauchlage beim plötzlichen Kindstod Stand der Diskussion. In: Althoff H (Hrsg): Rechtsmedizin von A - Z, Von der Arztethik bis zur zweifelhaften Vaterschaft, Kongressbericht der 74. Jahrestagung der Deutschen Gesellschaft für Rechtsmedizin, Verlag MurkenAltrogge Herzogenrath. S. 163

43. Kleemann WJ, Wiechern V, Schuck M, Töger HD (1995b) Intrathoracic and subconjunctival petechiae in sudden infant deah syndrome (SIDS). Forensic Sci Int 72(1):49-54

44. Kleemann WJ (1997a) Intrathorakale und subkonjunktivale Petechien bei Säuglingstodesfällen. Rechtsmed 7:139-46

45. Kleemann WJ, Poets CF (1997b) Der plötzliche Kindstod. Historische Betrachtungen. Pädiat Prax 52:223-30

46. Kleemenn WJ, Vock R, Bajanoswki T, Betz P, Bonte W, Freislederer A, Gerling I, Graw M, Hilgermann R, Höhmann E, Kleiber M, Lange E, Lasczekowski G, Lemke R, Leukel H, Madea B, Metter D, Pollak S, Ramms S, Saternus KS, Scheller M, Schmidt V, Schneider V, Six A, Springer E, Varchmin-Schultheiss K, Weiler G, Wessel B, Wilske J (1997c) Obduktionsfrequenz bei plötzlichen Kindstodfällen in der Bundesrepublik Deutschland in den Jahren von 1985 bis 1989. Rechtsmed 7:72-5

47. Kleemann WJ (2000a) Histologische Untersuchungen. In: Kurz R, Kenner Th, Poets Ch (Hrsg.): Der plötzliche Säuglingstod. Ein Ratgeber für Ärzte und Betroffene, Springer Verlag Wien New York. S. $71-81$

48. Kleemann WJ, Rognum TO (2000b): Makroskopische Befunde. In: Kurz R, Kenner Th, Poets Ch (Hrsg.): Der Plötzliche Säuglingstod. Ein Ratgeber für Ärzte und Betroffene, Springer Verlag Wien New York. S. 60 - 70 
49. Kohlendorfer U, Kiechl S, Sperl W (1998) Sudden infant death syndrome: risk factor profiles for distinct subgroups. Am J Epidemiol 147:960-8

50. Kopp JH (1830) Denkwuerdigkeiten der aerztlichen Praxis. 1. Band (Asthma thymicum), Frankfurt/ Main. S. 1-9

51.Kraus JF (1983) Methodologic Considerations in the Search for Risk Factors unique to Sudden Infant death Syndrome. In: Tildon JT, Roeder LM, Steinschneider A (Hrsg.): Sudden Infant death Syndrome, Academic Press New York London Paris

52. Krous HF, Jordan J (1984) Microscopic topography of introthoracic petechiae in sudden infant death syndrome. Arch Pathol Lab Med 108:77

53. Kurz R (2000) Apparent Live Threatening Events (ALTE). In: Kurz R, Kenner Th, Poets Ch (Hrsg.): Der plötzliche Säuglingstod. Ein Ratgeber für Ärzte und Betroffene, Springer Verlag Wien New York (2000). S. 145-150

54. Leach CE, Blair PS, Fleming PJ, Smith IJ, Platt MW, Berry PJ, Golding J (1999) Epidemiology of SIDS and explained sudden infant deaths, CESDI SUDI Research Group, Pediatrics 104(4):E43

55. Lohmann R (1996) Risikofaktoren beim plötzlichen Kindstod (SIDS), Berichte aus der Rechtswissenschaft. Shaker Verlag Aachen

56. Lorin de la Grandmaison G, Dorandeu A, Carton M, Patey A, Durigon M (1999) Increase of pulmonary density of macrophages in sudden infant death syndrome. Forensic Sci Int 104(2-3):179-87

57. Malloy MH, Freeman DH (2000) Birth Weight- and Gestational Age-Specific Sudden Infand Death Syndrome Mortality: United States, 1991 Versus 1995. Pediatrics 105(6):1227-31

58. Markestad T, Skadberg B, Hordvik E, Morild I, Irgens LM (1995) Sleeping position and sudden infant death syndrome (SIDS): Effect of an intervention programme to avoid prone sleeping. Acta Paediatr 84(4):375-8

59. Meyer H (1999) Zur Stabilität und Biogenese von Methanol und anderen Begleitstoffen in gelagerten Blutproben. Dissertationsschrift aus dem Institut für Rechtsmedizin der Ludwig-Maximilians-Universität München

60. Mitchell EA, Steward AW (1988) Deaths from sudden infant death syndrome on public holidays and weekends. Aus N Z J Med (Ausralia) 18(7):861-3

61. Mitchell EA, Steward AW (1997) Gender and the sudden infant death syndrome. New Zealand Cot Death Study Group. Acta Paediatr 86(8):854-6 
62. Mitchell EA, Thach BT, Thompson JM, Williams S (1999) Changing infants' sleep position increases risk of sudden infant death syndrome. New Zealand Cot Death Study. Arch Pediatr Adolesc Med 153(11):1136-41

63. Molz G, Hartmann HP, Michels L (1985) Plötzlicher Kindstod: Histologische Befunde in den Kopfspeicheldrüsen. Pathologe 6:8-12

64. Molz G, Brodzinowski A, Bär W, Vonlanthem B (1992) Morphologic variations in 180 cases of sudden infant death and 180 controls. Am J Forensic Med Pathol $13: 186-190$

65. Molz G (1998) Morphologie: Grenzwerte SID - nonSID. In: Saternus KS, Karimow S (Hrsg.): Säuglingssterblichkeit - Plötzlicher Kindstod (SID). Rechtsmedizinische Forschungsergebnisse, Schmidt-Römhild Lübeck, Band 18, S. 201-208

66. Molz G (2000) Der Plötzliche Säuglingstod in historischen Dokumenten, 2. Teil. In: Kurz R, Kenner Th, Poets Ch (Hrsg.): Der plötzliche Säuglingstod. Ein Ratgeber für Ärzte und Betroffene, Springer Verlag, Wien New York. S. 9-16

67. Murphy MF, Campbell MJ, Jones DR (1986) Increased risk of sudden infant death in older infants at weekends. Br Med J (Clin Res Ed) 293(6543):364-5

68. Naeye RL (1974) Hypoxemia and the Sudden Infant Death Syndrome. Science $186: 837-8$

69. Naeye RL (1990) Preventing the sudden infant death syndrome. Paediatr Perinat Epidemiol 4(1):12-21

70. Paltauf A (1890) Ueber die Beziehungen der Thymus zum plötzlichen Tod. Wiener Klinische Wochenschrift 2:172-5

71. Ogbuihi S, Zink P (1988) Pulmonary Lymphatics in SIDS - A comparative Morphometric study. Forensic Sci Int 39(2):197-206

72. Ostravsky Yu M (1986) Endogenous ethanol - ist metabolic, behavioral and biomedical significance. Alcohol 3(4):239-47

73. Penning R, Gilg Th, von Meyer L, Tutsch-Bauer E, Weichhold G (1996) Rechtsmedizin systematisch. UNI-MED Verlag Bremen Lorch. S. 55-57, 234

74. Peschel O, Bauer MF, Gilg Th, Meyer Lv (1992) Veränderung von Begleitstoffanalysen durch percutane Resorption propanolhaltiger Antiseptika. Blutalkohol Vol 29 Seiten 172-84

75. Platt MW, Blair PS, Fleming PJ, Smith IJ, Cole TJ, Leach CEA, Berry PJ, Golding J (2000) A clinical comparison of SIDS and explained sudden infant death: how healthy and how normal? Arch Dis Child 82(2):98-106 
76. Ponsonby AL, Dwyer T, Gibbons LE, Cochrane JA, Wang JG (1993) Factors potentiating the risk of sudden infant death syndrome associated with the prone position. N Eng J Med 329:377-82

77. Pschyrembel (1994) Klinisches Wörterbuch, 257. Auflage, de Gruyter Berlin New York

78. Püschel K, Hashimoto Y, Löning T, Lignitz E (1988) Cytomegalie der Kopfspeicheldrüsen bei SIDS. Z Rechtsmed 99:281-9

79. Rath G (1998) Mütterliche und exogene Risikofaktoren für einen plötzlichen Kindstod, In: Saternus KS, Karimow S (Hrsg): Säuglingssterblichkeit Plötzlicher Kindstod (SID), Rechtsmedizinische Forschungsergebnisse, Schmidt-Römhild Lübeck, Band 18, S. 163-166

80. Reh H (1979) Vitale Reaktionen der Atmungsorgane. Beitr Gerichtl Med $37: 121-6$

81. Riße M, Weiler G (1989) Differential diagnosis SIDS/ non-SIDS on the basis of histological findings of petechial thymus hemorrhages. Forensic Sci Int 43(1):17

82. Riße M, Weiler G (1989a) Comparative histologic studies of the origin of petechial thymus hemorrhage. Z Rechtsmed 102:33

83. Riße M (2001) Bedeutung und Wertigkeit morphologischer Befunde an Halsorganen unter forensisch-differentialdiagnostischen Aspekten. Eine rechtsmedizinische Studie zur Problematik des Plötzlichen Kindstodes (SIDS). Tectum Verlag Marburg

84. Rognum TO, Willinger M (1995) The story of the „Stavanger Definition“ In: Rognum TO (Hrsg.): Sudden Infant Death Syndrome. New Trends in the Nineties, Scandinavian University Press, Oslo Kopenhagen Stockholm, Kapitel 4, S. $21-25$

85. Rognum TO (2000a): Immunologische und mikrobiologische Untersuchungen. In: Kurz R, Kenner Th, Poets Ch (Hrsg.): Der plötzliche Säuglingstod. Ein Ratgeber für Ärzte und Betroffene. Springer Verlag Wien New York. S. 82-91

86. Rognum TO (2000b): Genetik und Molekularbiologie. In: Kurz R, Kenner Th, Poets Ch (Hrsg.): Der plötzliche Säuglingstod. Ein Ratgeber für Ärzte und Betroffene. Springer Verlag Wien New York. S. 92-97

87. Roll P (2000) Plötzlicher Säuglingstod und Kriminalität. In: Kurz R, Kenner Th, Poets Ch (Hrsg.): Der plötzliche Säuglingstod. Ein Ratgeber für Ärzte und Betroffene. Springer Verlag Wien New York. S. 107-115 
88. Russel-Jones DL (1985) Medical History - Sudden infant death in history and literature. Arch Dis Child 60:278-281

89. Saternus KS, Hebold K (1986) Anomalies of the vertebral artery in sudden infant death syndrome. Beitr Gerichtl Med 44:563-7

90. Schlaud R (1998) Epidemiologie des plötzlichen Kindstods (SID) In: Saternus KS, Karimow S (Hrsg): Säuglingssterblichkeit - Plötzlicher Kindstod (SID), Rechtsmedizinische Forschungsergebnisse: Schmidt-Römhild Lübeck; Band 18, S. 41-50

91. Schwatz PJ, Stramba Badiale M, Segantini A, Austoni P, Bosi G, Giorgetti R, Grancini F, Marni ED, Perticone F, Rosti D, Salice P (1998) Prolongation of the QT interval and the sudden infant death syndrome. N Engl J Med 338:1709-14

92. Sperl W (2000) Der plötzliche Säuglingstod und Stoffwechselstörungen. In: Kurz R, Kenner Th, Poets Ch (Hrsg.): Der plötzliche Säuglingstod. Ein Ratgeber für Ärzte und Betroffene. Springer Verlag Wien New York. S. 99-106

93. Spiers PS, Lohmann R, Guntheroth WG (1993) Birth order and risk of sudden infant death syndrome: Is the time relationshion negative? J Paediatr Child Health 29(3):215-8

94. Spiers PS, Guntheroth WG (1999) The Effect of the Weekend on the Risk of Sudden Infant Death Syndrome. Pediatrics 104(5):E58

95. Sprung R, Bonte W, Rudell E (1983) Determination of congener substances of alcoholic beverages and their metabolites in blood and urine samples. Beitr Gerichtl Med 41:219-22

96. Standfast SJ, Jereb S, Aliferis D, Janerich DT (1983) Epidemiology of SIDS in Upstate New York. In: Tildon JT, Roeder LM, Steinschneider A (Hrsg.): Sudden Infant Death Syndrome. Academic Press New York London Paris

97. Statistisches Bundesamt (1992, 1994, 1996, 1998, 2000) Statistisches Jahrbuch. Metzler-Poeschel Wiesbaden Stuttgart

98. Steinschneider A (1972) Prolonged apnea and the sudden infant death syndrome: clinical and laboratory observations, Pediatrics 50:646-54

99. Taylor JA, Sanderson M (1995) A reexamination of the risk factors for the sudden infant death syndrome. J Pediatr 126(6):887-91

100. Taylor JA, Krieger JW, Reay DT, Davis RL, Harruff R, Cheney LK (1996) Prone sleep position and the sudden infant death syndrome in King County, Washington: a case-control study. J Pediatr 128(5Pt1):626-30 
101. Telford DR, Morris JA, Hughes P (1989) The nasopharyngeal bacterial flora in the sudden infant death syndrome. J Infec 18(2):125-30

102. Trowitzsch E, Meyer G, Schluter B, Buschatz D, Andler W (1992) "A life threatening event" in infants. Results of polysomnography and examination of a group of 122 infants. Monatsschr Kinderheilkd 140(4):233-6

103. Valdes-Dapena M (1991) A pathologist's perspective on the sudden infant death syndrome. Pathol Annu 27(part I):133-64

104. Valdes-Dapena M (1992) The sudden infant death syndrome: Pathologic Findings. Clin Perinat 19(4):701-16

105. Valdes-Dapena M, McFeeley PA, Hoffmann HJ, Damus KH, Franciosi RR, Allison DJ, Jones M, Hunter JC (1993) Histopathology Atlas for the Sudden Infant Death Syndrome. Armed Forces Institute of Pathology, Washington D.C.

106. Westfälische Wilhelms-Universität Münster (2000) Plötzlicher Säuglingstod - Eine multizentrische Studie unter Leitung der Westfälischen Wilhelms-Universität Münster. Studienhandbuch Hauptphase 1998 - 2003 (2000), Stand: 28.02.2000

107. Williams SM, Mitchell EA, Scragg R (1997) Why is sudden infant death syndrome more common at weekends? The New Zealand National Cot Death Study Group. Arch Dis Child 77(5):415-9

108. Willinger M, James LS, Catz C (1991) Defining the sudden infant death syndrome (SIDS): Deliberations of an Expert Panel Convened by the National Institute of Child Health and Human Development. Pediatr Pathol 11:677-84

109. Wilske J (1984) Der Plötzliche Säuglingstod - Morphologische Abgrenzung, Pathomechanismus und Folgerungen für die Praxis. Springer Verlag Berlin Heidelberg New York

110. Wittmann S (1992) Isopropanol- und Acetonspiegel im Serum nach präoperativer Flächendesinfektion mit isopropanolhaltigen Antiseptika. Blutalkohol Vol.29/1992, Seiten 326-35

111. Wittmann S (1996) Serumspiegel von Methanol und anderen Begleitstoffen nach Obstaufnahme ohne und mit Ethanolbelastung. Dissertationsschrift aus dem Institut für Rechtsmedizin der LudwigMaximilians-Universität München

112. World Health Organisation (2000) World health statistics annual. WHO

113. Young M, Turnbull HM (1931) An analysis of the data collected by the status lymphaticus investigation commitee. J Path Bact 34:213-57 


\section{ANHANG}

Abb.24:

\section{SIDS-Studie}

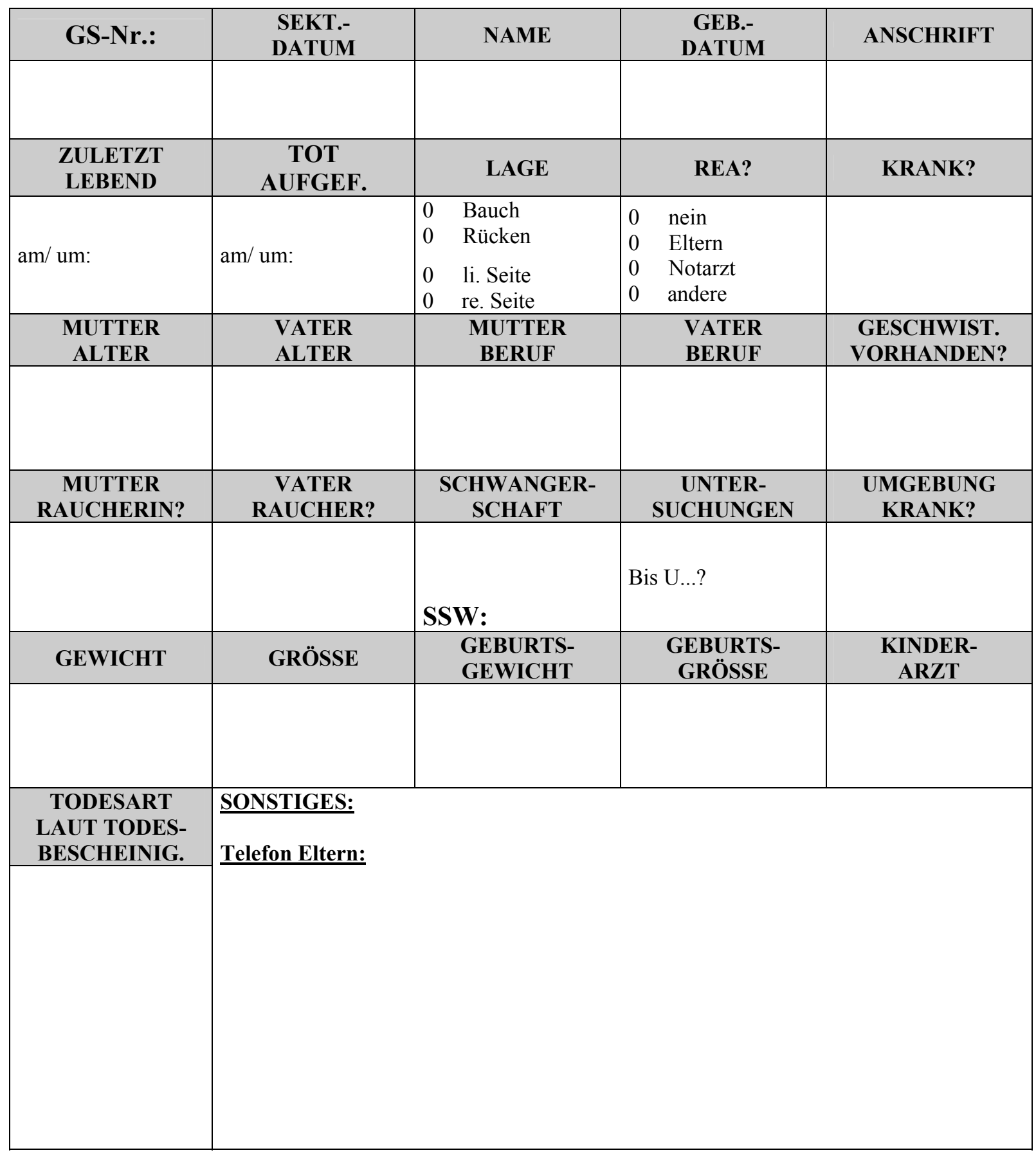




\section{Histologische Färbungen}

\section{HE (Hämalaun-Eosin):}

entparaffinieren in Xylol (15 min)

absteigende Alkoholreihe (100\%, 96\%, 80\%, 70\%; jeweils ca. 1 min)

Aua dest. (5 min.)

Hämalaun (10 min)

wässern (10 min)

Eosin 2\% (15 min)

wässern (5 min)

aufsteigende Alkoholreihe $(70 \%, 80 \%, 96 \%, 100 \%)$

Xylol (10 min), eindecken

\section{EvG (Elastica van Giesson)}

entparaffinieren in Xylol (15 min)

absteigende Alkoholreihe (100\%, 96\%, 80\%, 70\%; jeweils ca. $1 \mathrm{~min})$

Resorcin-Fuchsin (25 min) im Wärmeschrank

drei mal in 70\% Alkohol spülen

Weigerts Eisenhämatoxylin (10 min)

wässern (20 min)

Pircofuchsin (5 min)

kurz wässern

aufsteigende Alkoholreihe $(70 \%, 80 \%, 96 \%, 100 \%)$

Xylol (10 min), eindecken

\section{PAS}

entparaffinieren in Xylol (15 min)

absteigende Alkoholreihe (100\%, 96\%, 80\%, 70\%; jeweils ca. 1 min)

Perjodsäure $1 \%$ (10 min)

Alkohol 70\% (10 min)

Schiffsches Reagens (15 min)

differenzieren mit Kaliumdisulfid 0,5\%

Alkohol 70\% (drei mal je ca. $1 / 2 \mathrm{~min}$ )

aufsteigende Alkoholreihe $(80 \%, 96 \%, 100 \%)$

Xylol (10 min), eindecken

\section{Alzianblau-PAS}

entparaffinieren in Xylol (15 min)

absteigende Alkoholreihe $(100 \%, 96 \%, 80 \%, 70 \%$; jeweils ca. $1 \mathrm{~min})$

Alzianblau (30 min)

wässern (10 min)

Perjodsäure $1 \%$ (10 min)

Alkohol 70\% (10 min)

Schiffsches Reagens (15 min)

differenzieren mit Kaliumdisulfid 0,5\%

Alkohol 70\% (drei mal je ca. $1 / 2 \mathrm{~min}$ )

aufsteigende Alkoholreihe (80\%, 96\%, 100\%)

Xylol (10 min), eindecken 


\section{Fe (Eisen)}

entparaffinieren in Xylol (15 min)

absteigende Alkoholreihe $(100 \%, 96 \%, 80 \%, 70 \%$; jeweils ca. $1 \mathrm{~min})$

Aqua dest. (1 min)

färben in 1:1-Gemisch aus Kaliumferrocyanid 2\% und Salzsäure 2,5\% (60 min)

wässern (10 min)

Kernechtrot (10 min)

wässern (10 min)

aufsteigende Alkoholreihe $(80 \%, 96 \%, 100 \%)$

Xylol (10 min), eindecken

\section{Giemsa}

entparaffinieren in Xylol (15 min)

absteigende Alkoholreihe (100\%, 96\%, 80\%, 70\%; jeweils ca. $1 \mathrm{~min})$

Aqua dest. (5 min)

Giemsalösung 2\% (12 Stunden)

wässern (10 min)

Gemisch aus 95\% Aceton und 5\% Xylol (kurz spülen)

Gemisch aus 70\% Aceton und 30\% Xylol (kurz spülen)

Gemisch aus 30\% Aceton und 70\% Xylol (kurz spülen)

Xylol (10 min), eindecken

\section{Ölrot}

Aqua dest (10 min)

2-Propanol 60\% (5 min)

färben in Ölrotlösung $60 \%$

differenzieren in 2-Propanol 60\%

Aqua dest. (kurz spülen)

Hämalaun (10 min)

Aqua dest. (10 min)

eindecken mit Glyceringelatine 


\section{Gesamtdaten}

Tabelle 77:

\begin{tabular}{|c|c|c|c|c|c|c|c|c|c|c|}
\hline GS-Nr. & \begin{tabular}{|c|} 
Kalender \\
monat d. \\
Todes
\end{tabular} & $\begin{array}{l}\text { Alter } \\
\text { (Mo.) }\end{array}$ & $w / m$ & $\begin{array}{l}\text { Posi- } \\
\text { tion }\end{array}$ & \begin{tabular}{|c|} 
Gewich + \\
Größet: \\
Geburt
\end{tabular} & \begin{tabular}{|c|} 
Gewicht + \\
Größe: \\
Tod
\end{tabular} & $\begin{array}{l}\text { Alter: } \\
\text { Vater }\end{array}$ & $\begin{array}{l}\text { Alter: } \\
\text { Mutter }\end{array}$ & ssw & krank/ gesund \\
\hline $171 / 99$ & 1 & 4 & $w$ & Seite & $3890 / 52$ & $5100 / 59$ & 33 & 26 & 42 & gesund \\
\hline $175 / 99$ & 1 & 4 & $m$ & Seite & $3240 / 48$ & $5500 / 65$ & 31 & 29 & \# & gesund \\
\hline $181 / 99$ & 1 & 5 & $\mathrm{~m}$ & Bauch & $\#$ & $6600 / 66$ & 23 & 20 & $\#$ & krank \\
\hline $262 / 99$ & 2 & 9 & w & Bauch & $3420 / 53$ & $8600 / 76$ & 34 & 23 & 40 & gesund \\
\hline $284 / 99$ & 2 & 10 & $w$ & $\#$ & $7200 / 74$ & $\#$ & $\#$ & $\#$ & $\#$ & krank \\
\hline $511 / 99$ & 3 & 5 & w & Seite & $\#$ & $5600 / 60$ & $\#$ & 29 & 39 & seit $1 \mathrm{Wo}$ \\
\hline $602 / 99$ & 4 & 9 & $w$ & Bauch & $3600 / 54$ & $8500 / 75$ & 36 & 33 & 39 & krank \\
\hline $760 / 99$ & 4 & 3 & $w$ & Bauch & $3950 / 56$ & $4600 / 61$ & 36 & 36 & & gesund \\
\hline $781 / 99$ & 5 & 8 & $m$ & Rücken & \# & $7800 / 70$ & \# & \# & \# & $\#$ \\
\hline $852 / 99$ & 5 & 6 & $m$ & Bauch & $3000 / 49$ & $7300 / 68$ & 21 & 18 & 39 & gesund \\
\hline $914 / 99$ & 5 & 7 & $m$ & Rücken & $625 / 31$ & $4700 / 60$ & 25 & 28 & 25 & gesund \\
\hline $950 / 99$ & 5 & 4 & $w$ & Rücken & $3170 / 50$ & $5300 / 61$ & 19 & 17 & 39 & krank \\
\hline $981 / 99$ & 6 & 5 & $m$ & Bauch & $3200 / 51$ & $6500 / 64$ & 32 & 30 & 36 & krank \\
\hline $1018 / 99$ & 6 & 9 & $w$ & Bauch & $2990 / 49$ & $6900 / 69$ & 27 & 25 & $\#$ & seit $1 \mathrm{Wo}$ \\
\hline $1079 / 99$ & 6 & 3 & $m$ & Bauch & $3700 / 56$ & $5500 / 62$ & $\#$ & $\#$ & 40 & seit 1 Wo \\
\hline $1080 / 99$ & 6 & 2 & $w$ & $\#$ & $3140 / 51$ & $4100 / 55$ & $\#$ & 30 & $\#$ & gesund \\
\hline $1155 / 99$ & 7 & 1 & $\mathrm{~m}$ & Seite & $2620 / 51$ & $2600 / 53$ & 30 & 18 & $\#$ & krank \\
\hline $1184 / 99$ & 7 & 3 & $m$ & Rücken & $3570 / 52$ & $5600 / 62$ & 52 & 33 & $\#$ & gesund \\
\hline $1195 / 99$ & 7 & 9 & $w$ & Bauch & $3470 / 52$ & $7000 / 73$ & 33 & 27 & $\#$ & gesund \\
\hline $1258 / 99$ & 7 & 11 & $m$ & Rücken & $2920 / 50$ & $8900 / 74$ & \# & 32 & $\#$ & krank \\
\hline $1459 / 99$ & 8 & 10 & $w$ & Bauch & $3040 / 49$ & $8100 / 75$ & 22 & 20 & 39 & gesund \\
\hline $1461 / 99$ & 8 & 11 & $w$ & $\#$ & $2930 / 46$ & $11500 / 85$ & $\#$ & 20 & 39 & gesund \\
\hline $1537 / 99$ & 9 & 4 & $w$ & Bauch & $3520 / 55$ & $6200 / 65$ & 32 & 19 & $\#$ & gesund \\
\hline $1624 / 99$ & 9 & 4 & $\mathrm{~m}$ & Bauch & $2290 / 46$ & $6000 / 56$ & 24 & 25 & $\#$ & gesund \\
\hline $1625 / 99$ & 9 & 9 & $w$ & Bauch & $2710 / 47$ & $7600 / 67$ & 34 & 22 & $\#$ & seit 1 Wo \\
\hline $1645 / 99$ & 9 & 7 & $w$ & Rücken & 1070/? & $4500 / 61$ & 19 & 20 & $\#$ & krank \\
\hline $1685 / 99$ & 10 & 9 & $w$ & Rücken & $3040 / 48$ & $7200 / 67$ & $\#$ & 32 & $\#$ & gesund \\
\hline $1821 / 99$ & 10 & 7 & $m$ & Bauch & $1840 / 47$ & $7800 / 69$ & 28 & 18 & 33 & gesund \\
\hline $1862 / 99$ & 11 & 8 & $m$ & Bauch & $2310 / 48$ & $5900 / 69$ & 29 & 21 & 37 & gesund \\
\hline $1891 / 99$ & 11 & 10 & $w$ & Bauch & $3370 / 50$ & $7300 / 71$ & $\#$ & 25 & 40 & krank \\
\hline $1961 / 99$ & 11 & 5 & $\mathrm{~m}$ & Rücken & $2700 / 49$ & $6300 / 65$ & 33 & 33 & 39 & gesund \\
\hline $2080 / 99$ & 12 & 11 & $w$ & Rücken & $2140 / 46$ & $5500 / 65$ & 32 & 32 & $\#$ & gesund \\
\hline $2133 / 99$ & 12 & 4 & $\mathrm{~m}$ & $\#$ & $3830 / 53$ & $5100 / 64$ & 24 & 20 & $\#$ & krank \\
\hline $2139 / 99$ & 12 & 7 & $\mathrm{~m}$ & $\#$ & $\#$ & $6700 / 69$ & 34 & 20 & $\#$ & gesund \\
\hline $2160 / 99$ & 12 & 1 & $w$ & Bauch & $1895 / 41$ & $2527 / 47$ & $\#$ & 29 & $\#$ & gesund \\
\hline $59 / 00$ & 1 & 3 & $w$ & Bauch & $3040 / 50$ & $4430 / 60$ & 23 & 21 & 41 & seit $1 \mathrm{Wo}$ \\
\hline $133 / 00$ & 1 & 10 & $\mathrm{~m}$ & Bauch & $3410 / 53$ & $8600 / 76$ & $\#$ & 31 & 36 & krank \\
\hline $161 / 00$ & 1 & 9 & $m$ & Bauch & $4030 / 53$ & $7700 / 72$ & 47 & 29 & 40 & krank \\
\hline $232 / 00$ & 2 & 10 & $w$ & Rücken & $3120 / 47$ & $8600 / 73$ & $\#$ & 27 & 40 & krank \\
\hline $275 / 00$ & 2 & 3 & $w$ & Bauch & $2470 / 45$ & $4200 / 56$ & 25 & 22 & 38 & gesund \\
\hline $381 / 00$ & 2 & 2 & $m$ & Bauch & $3430 / 51$ & $4706 / 57$ & 33 & 30 & 40 & gesund \\
\hline $393 / 00$ & 2 & 4 & $m$ & Bauch & $3850 / 53$ & $5700 / 63$ & 39 & 35 & 39 & gesund \\
\hline $413 / 00$ & 2 & 2 & $m$ & Rücken & $3050 / 47$ & $3900 / 53$ & $\#$ & 29 & 37 & krank \\
\hline $442 / 00$ & 2 & 1 & $\mathrm{~m}$ & Rücken & $3300 / 51$ & $4300 / 54$ & 30 & 35 & 40 & gesund \\
\hline
\end{tabular}




\begin{tabular}{|c|c|c|c|c|c|c|c|c|c|c|}
\hline GS-Nr. & \begin{tabular}{|c} 
Kalender \\
monat d. \\
Todes
\end{tabular} & $\begin{array}{l}\text { Alter } \\
\text { (Mo.) }\end{array}$ & $w / m$ & $\begin{array}{l}\text { Posi- } \\
\text { tion }\end{array}$ & $\begin{array}{l}\text { Gewich/ } \\
\text { Größe: } \\
\text { Geburt }\end{array}$ & $\begin{array}{c}\text { Gewicht/ } \\
\text { Größe: } \\
\text { Tod }\end{array}$ & $\begin{array}{l}\text { Alter: } \\
\text { Vater }\end{array}$ & $\begin{array}{l}\text { Alter: } \\
\text { Mutter }\end{array}$ & ssw & krank/ gesund \\
\hline $500 / 00$ & 3 & 4 & $\mathrm{~m}$ & Rücken & $2950 / 50$ & $5500 / 62$ & 28 & 19 & $\#$ & krank \\
\hline $759 / 00$ & 4 & 11 & $\mathrm{~m}$ & Bauch & $3080 / 49$ & $6900 / 73$ & 28 & 28 & 39 & krank \\
\hline $824 / 00$ & 4 & 3 & w & Bauch & $3360 / 51$ & $3800 / 56$ & 36 & 35 & 41 & gesund \\
\hline $896 / 00$ & 5 & 6 & $\mathrm{~m}$ & Bauch & $3480 / 51$ & $7100 / 68$ & 19 & 18 & 39 & krank \\
\hline $986 / 00$ & 5 & 12 & $\mathrm{~m}$ & $\#$ & $830 / 36$ & $8800 / 73$ & $\#$ & 19 & 27 & krank \\
\hline $1015 / 00$ & 5 & 11 & $w$ & Bauch & $4010 / 54$ & $9400 / 77$ & 35 & 34 & 40 & gesund \\
\hline $1069 / 00$ & 6 & 2 & $m$ & $\#$ & $4000 / 52$ & $\#$ & $\#$ & 31 & 40 & gesund \\
\hline $1134 / 00$ & 6 & 4 & $\mathrm{~m}$ & $\#$ & $1500 / 41$ & $5400 / 55$ & 27 & 21 & 33 & krank \\
\hline $1331 / 00$ & 7 & 3 & $\mathrm{~m}$ & $\#$ & $3270 / 52$ & $6300 / 62$ & $\#$ & 28 & 39 & gesund \\
\hline $1333 / 00$ & 7 & 3 & $m$ & Bauch & $2410 / 46$ & $4118 / 56$ & 27 & 24 & 36 & $\#$ \\
\hline $1371 / 00$ & 7 & 3 & w & Bauch & $\#$ & $\#$ & 27 & 25 & 36 & gesund \\
\hline $1687 / 00$ & 9 & 8 & $w$ & Rücken & $3640 / 53$ & $8300 / 75$ & 38 & 34 & 40 & krank \\
\hline $1768 / 00$ & 10 & 4 & $m$ & Bauch & $2790 / 51$ & $5400 / 62$ & $\#$ & 31 & 39 & gesund \\
\hline $1789 / 00$ & 10 & 10 & w & Rücken & $4650 / 57$ & $7800 / 75$ & 34 & 33 & $\#$ & krank \\
\hline $1814 / 00$ & 10 & 2 & $w$ & $\#$ & $2950 / 50$ & $3914 / 56$ & 32 & 29 & $\#$ & gesund \\
\hline $1857 / 00$ & 10 & 10 & $\mathrm{~m}$ & Rücken & $3780 / 52$ & $10300 / 78$ & $\#$ & 22 & 40 & gesund \\
\hline $2116 / 00$ & 11 & 5 & $w$ & Bauch & $3470 / 52$ & $6600 / 66$ & 32 & 26 & 39 & gesund \\
\hline $2180 / 00$ & 12 & 3 & $w$ & Bauch & $3470 / 50$ & $6900 / 62$ & 35 & 29 & $\#$ & krank \\
\hline $2201 / 00$ & 12 & 11 & $w$ & Bauch & $1670 / 47$ & $6700 / 62$ & \# & 41 & 36 & krank \\
\hline $138 / 01$ & 1 & 5 & $m$ & Bauch & $3510 / 48$ & $7200 / 65$ & 28 & 27 & 41 & krank \\
\hline $145 / 01$ & 1 & 1 & $\mathrm{~m}$ & $\#$ & $\#$ & $5000 / 56$ & $\#$ & 18 & $\#$ & krank \\
\hline $309 / 01$ & 2 & 5 & $w$ & Bauch & $2450 / 47$ & $6300 / 65$ & \# & 23 & 37 & krank \\
\hline $381 / 01$ & 2 & 8 & $\mathrm{~m}$ & Bauch & $3110 / 51$ & $6800 / 70$ & $\#$ & 21 & 40 & krank \\
\hline $493 / 01$ & 3 & 2 & $\mathrm{~m}$ & Bauch & $3610 / 51$ & $4600 / 59$ & 24 & 19 & 39 & gesund \\
\hline $565 / 01$ & 3 & 5 & $w$ & Bauch & $3360 / 51$ & $5700 / 63$ & 27 & 18 & 39 & gesund \\
\hline $576 / 01$ & 3 & 5 & $\mathrm{~m}$ & Bauch & $\#$ & $7500 / 67$ & $\#$ & 28 & $\#$ & krank \\
\hline $801 / 01$ & 5 & 7 & $m$ & Bauch & $2710 / 51$ & $6100 / 65$ & $\#$ & 26 & 40 & krank \\
\hline $889 / 01$ & 5 & 10 & $\mathrm{~m}$ & Bauch & $2810 / 48$ & $8900 / 74$ & 33 & 31 & 37 & $\#$ \\
\hline $961 / 01$ & 5 & 3 & $\mathrm{~m}$ & $\#$ & $3250 / 51$ & $5800 / 63$ & \# & 23 & $\#$ & gesund \\
\hline $980 / 01$ & 6 & 4 & $\mathrm{~m}$ & Bauch & $3460 / 52$ & $6600 / 64$ & 28 & 25 & 38 & krank \\
\hline $1096 / 01$ & 6 & 7 & $w$ & Rücken & $3120 / 53$ & $6700 / 69$ & 37 & 26 & 40 & gesund \\
\hline $1154 / 01$ & 6 & 6 & $w$ & $\#$ & $3090 / 50$ & $6000 / 67$ & 27 & 20 & 41 & $\#$ \\
\hline $1171 / 01$ & 6 & 2 & $w$ & Bauch & $3300 / 49$ & $4100 / 54$ & 34 & 20 & 38 & gesund \\
\hline $1355 / 01$ & 7 & 6 & w & Rücken & $550 / ?$ & $3400 / 53$ & $\#$ & 40 & 30 & gesund \\
\hline $1456 / 01$ & 8 & 1 & $\mathrm{~m}$ & Rücken & $\#$ & $3100 / 56$ & $\#$ & 29 & $\#$ & krank \\
\hline $1554 / 01$ & 8 & 6 & $\mathrm{~m}$ & Bauch & $3160 / 52$ & $7700 / 69$ & $\#$ & 26 & 39 & $\#$ \\
\hline $1746 / 01$ & 9 & 9 & $\mathrm{~m}$ & Rücken & $3740 / 52$ & $8700 / 74$ & 38 & 36 & 41 & gesund \\
\hline $1923 / 01$ & 10 & 4 & $\mathrm{~m}$ & Bauch & $3100 / 50$ & $3100 / 50$ & $\#$ & 27 & 37 & gesund \\
\hline
\end{tabular}


Tabelle 78:

\begin{tabular}{|c|c|c|c|c|c|c|c|c|c|c|c|c|c|c|c|c|c|c|c|c|c|}
\hline GS-Nr. & $\begin{array}{l}\text { Todesart It. } \\
\text { Todesbe- } \\
\text { scheinigung }\end{array}$ & $\begin{array}{l}\text { Bakterio- } \\
\text { logie }\end{array}$ & $\begin{array}{l}\text { Viro- } \\
\text { logie }\end{array}$ & $\begin{array}{l}\text { Kate- } \\
\text { gorie }\end{array}$ & \multicolumn{17}{|c|}{ Histologische Befunde } \\
\hline $171 / 99$ & nicht aufgekl. & 0 & CMV & 2 & 1 & & & & \begin{tabular}{l|l}
0 & \\
\end{tabular} & & 0 & 0 & 11 & 12 & 0 & 0 & 0 & 0 & 0 & 0 & 0 \\
\hline $175 / 99$ & nicht aufgekl. & Staph & 0 & 4 & 1 & $2 \sqrt{3}$ & 0 & 0 & 0 & \begin{tabular}{l|l}
0 & 0
\end{tabular} & 0 & 0 & 0 & 0 & 0 & 0 & 0 & 0 & 0 & 0 & 0 \\
\hline 181/99 & \# & 0 & 0 & 2 & 1 & 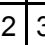 & 0 & 0 & 0 & \begin{tabular}{l|l}
0 & 0 \\
\end{tabular} & 0 & 0 & 0 & 0 & 0 & 0 & 0 & 0 & 0 & 0 & 0 \\
\hline $262 / 99$ & nicht aufgekl. & 0 & 0 & 4 & 1 & $0 \sqrt[3]{3}$ & 4 & 0 & 6 & \begin{tabular}{l|l}
0 & 0 \\
\end{tabular} & 0 & 0 & 11 & 12 & 0 & 0 & 0 & \begin{tabular}{|l|}
0 \\
\end{tabular} & \begin{tabular}{|l|}
0 \\
\end{tabular} & 0 & 0 \\
\hline $284 / 99$ & $\#$ & 0 & 0 & 2 & 1 & 23 & 0 & 0 & 0 & \begin{tabular}{l|l}
0 & 0
\end{tabular} & ) & 0 & 11 & 0 & 0 & 14 & 0 & 0 & 0 & 0 & 0 \\
\hline $511 / 99$ & nicht aufgekl. & 0 & 0 & 2 & 1 & 23 & 0 & 0 & 0 & \begin{tabular}{l|l}
0 & 0
\end{tabular} & 0 & 0 & 11 & 0 & 0 & 0 & 0 & 0 & 0 & 0 & 0 \\
\hline $602 / 99$ & nicht aufgekl. & 0 & 0 & 2 & 1 & $2 \sqrt[3]{3}+a+a$ & 0 & 0 & 6 & \begin{tabular}{l|l}
0 & 0
\end{tabular} & 0 & 0 & 11 & 0 & 0 & 0 & 0 & 0 & 0 & 0 & 0 \\
\hline $760 / 99$ & $\#$ & 0 & 0 & 1 & 0 & 0 c & 0 & 0 & 0 & \begin{tabular}{l|l}
0 & 0
\end{tabular} & 0 & 0 & 0 & 0 & 0 & 0 & 0 & 0 & 0 & 0 & 0 \\
\hline $781 / 99$ & nicht aufgekl. & Staph & 0 & 2 & 0 & $0] 0$ & 0 & 0 & 0 & \begin{tabular}{l|l}
0 & 0
\end{tabular} & 0 & 0 & 11 & 12 & 0 & 0 & 0 & \begin{tabular}{|l|}
0 \\
\end{tabular} & 0 & 0 & 0 \\
\hline $852 / 99$ & $\#$ & 0 & 0 & 4 & 0 & 0 co & 4 & 5 & 0 & \begin{tabular}{l|l}
0 & 0
\end{tabular} & 0 & 0 & 0 & 0 & 0 & 0 & 0 & 0 & 0 & 0 & 0 \\
\hline $914 / 99$ & \# & Staph & 0 & 2 & 0 & $0 \sqrt[3]{3}$ & 0 & 0 & 0 & \begin{tabular}{l|l}
0 & 0 \\
\end{tabular} & 0 & 0 & 0 & 0 & 0 & 0 & 15 & 0 & 0 & 0 & 0 \\
\hline 950/99 & nicht nat. & 0 & CMV & 3 & 0 & $0 \sqrt[3]{3}$ & 4 & 5 & 0 & \begin{tabular}{l|l}
7 & 0 \\
\end{tabular} & 0 & 0 & 11 & 12 & 0 & 0 & 0 & 0 & 0 & 0 & 0 \\
\hline $981 / 99$ & nicht aufgekl. & Strept & 0 & 2 & 0 & $2 \sqrt{2}$ & 0 & 5 & 0 & $0 \mid 0$ & 0 & 0 & 11 & 12 & 0 & 0 & 0 & \begin{tabular}{|l|}
0 \\
\end{tabular} & 0 & 0 & 0 \\
\hline $1018 / 99$ & $\#$ & 0 & 0 & 2 & 1 & 0 c & 4 & 5 & 0 & \begin{tabular}{l|l}
7 & 0
\end{tabular} & 0 & 0 & 11 & 12 & 0 & 0 & 0 & 0 & 0 & 0 & 0 \\
\hline $1079 / 99$ & $\#$ & Staph & 0 & 2 & 1 & $0 \sqrt[3]{3}$ & 0 & 0 & 0 & \begin{tabular}{l|l}
0 & 0 \\
\end{tabular} & 0 & 0 & 0 & 0 & 0 & 0 & 0 & 0 & 0 & 0 & 0 \\
\hline $1080 / 99$ & nicht aufgekl. & 0 & 0 & 4 & 0 & 00 & 0 & 0 & 0 & \begin{tabular}{l|l}
0 & 0
\end{tabular} & 0 & 0 & 0 & 0 & 0 & 0 & 0 & 0 & 0 & 0 & 0 \\
\hline $1155 / 99$ & nicht aufgekl. & 0 & 0 & 2 & 0 & 00 & 0 & 0 & 0 & \begin{tabular}{l|l}
0 & 8 \\
\end{tabular} & 0 & 0 & 0 & 0 & 0 & 0 & 0 & 0 & 0 & 0 & 0 \\
\hline $1184 / 99$ & nicht aufgekl. & Kleb & Ado & 2 & 0 & 03 & 4 & 0 & 6 & \begin{tabular}{l|l}
0 & 0 \\
\end{tabular} & 0 & 0 & 0 & 0 & 0 & 0 & 0 & 0 & 0 & 0 & 0 \\
\hline $1195 / 99$ & nicht nat. & Staph & 0 & 1 & 0 & 0 c & 0 & 0 & 0 & \begin{tabular}{l|l}
0 & 0
\end{tabular} & 0 & 0 & 0 & 0 & 0 & 0 & 0 & 0 & 0 & 0 & 0 \\
\hline $1258 / 99$ & nicht aufgekl. & 0 & $\mathrm{~Pa} 3$ & 4 & 1 & $0 \sqrt[3]{3}$ & 0 & 0 & 6 & \begin{tabular}{l|l}
0 & 0 \\
\end{tabular} & 0 & 0 & 0 & 0 & 0 & 0 & 0 & 0 & 0 & 0 & 0 \\
\hline $1459 / 99$ & nicht aufgekl. & 0 & 0 & 2 & 1 & $0 \sqrt[3]{3}$ & 0 & 0 & 0 & \begin{tabular}{l|l}
0 & 0
\end{tabular} & 0 & 0 & 0 & 0 & 0 & 0 & 0 & 0 & 0 & 0 & 0 \\
\hline $1461 / 99$ & nicht nat. & 0 & 0 & 4 & 0 & $0 \sqrt[3]{3}$ & 0 & 0 & 0 & \begin{tabular}{l|l}
0 & 0
\end{tabular} & 0 & 0 & 0 & 0 & 0 & 0 & 0 & 0 & 0 & 0 & 0 \\
\hline $1537 / 99$ & nicht aufgekl. & Staph & 0 & 2 & 1 & $2 \sqrt{3}$ & \begin{tabular}{l|l}
30 \\
\end{tabular} & 0 & 0 & \begin{tabular}{l|l}
0 & 0
\end{tabular} & 0 & 0 & 11 & 0 & 0 & 0 & 0 & 0 & 0 & 0 & 0 \\
\hline $1624 / 99$ & nicht aufgekl. & 0 & CMV & 2 & 1 & 23 & \begin{tabular}{|l|l}
3 & 4
\end{tabular} & 5 & 0 & \begin{tabular}{l|l}
0 & 0
\end{tabular} & 0 & 0 & 0 & 0 & 0 & 0 & 0 & 0 & 0 & 0 & 0 \\
\hline $1625 / 99$ & nicht aufgekl. & 0 & 0 & 2 & 0 & 00 & 0 & 0 & 0 & \begin{tabular}{l|l}
0 & 0 \\
\end{tabular} & 0 & 0 & 11 & 12 & 0 & 0 & 0 & 0 & 0 & 0 & 0 \\
\hline $1645 / 99$ & nicht aufgekl. & 0 & Ent & 2 & 0 & 00 & \begin{tabular}{|l|l}
3 & 4 \\
\end{tabular} & 0 & 0 & \begin{tabular}{l|l}
0 & 0
\end{tabular} & 0 & 0 & 0 & 0 & 0 & 0 & 0 & 0 & 0 & 0 & 0 \\
\hline $1685 / 99$ & nicht aufgekl. & Staph & 0 & 2 & 1 & 00 & 0 & 0 & 0 & \begin{tabular}{l|l}
0 & 0 \\
\end{tabular} & 0 & 0 & 0 & 0 & 0 & 0 & 0 & 0 & 0 & 0 & 0 \\
\hline $1821 / 99$ & nicht aufgekl. & Pneu & CMV & 3 & 0 & 23 & \begin{tabular}{|l|l}
3 & 4 \\
\end{tabular} & 5 & 0 & \begin{tabular}{l|l}
7 & 8 \\
\end{tabular} & 0 & 0 & 11 & 12 & 0 & 0 & 0 & 16 & 0 & 0 & 0 \\
\hline $1862 / 99$ & nicht aufgekl. & 0 & 0 & 2 & 1 & 00 & 0 & 0 & 0 & \begin{tabular}{l|l}
0 & 0
\end{tabular} & 0 & 0 & 0 & 0 & 0 & 0 & 0 & 0 & 0 & 0 & 0 \\
\hline $1891 / 99$ & nicht aufgekl. & 0 & 0 & 2 & 1 & $0 \sqrt[3]{3}$ & \begin{tabular}{|l|l|}
30 \\
\end{tabular} & 0 & 0 & \begin{tabular}{l|l}
0 & 0
\end{tabular} & 0 & 0 & 0 & 0 & 0 & 0 & 0 & \begin{tabular}{|l|}
0 \\
\end{tabular} & \begin{tabular}{|l|}
0 \\
\end{tabular} & 0 & 0 \\
\hline 1961/99 & nicht nat. & Pneu & 0 & 2 & 1 & 0 C & 4 & 0 & 0 & \begin{tabular}{l|l}
0 & 0
\end{tabular} & 0 & 0 & 0 & 0 & 0 & 0 & 0 & 0 & 0 & 0 & 0 \\
\hline $2080 / 99$ & nicht nat. & 0 & 0 & 4 & 1 & $0] c$ & 0 & 0 & 0 & \begin{tabular}{l|l}
0 & 0
\end{tabular} & 0 & 0 & 0 & 0 & 0 & 0 & 0 & 0 & 0 & 0 & 0 \\
\hline $2133 / 99$ & nicht aufgekl. & 0 & 0 & 2 & 0 & 0 C & 0 & 0 & 0 & \begin{tabular}{l|l}
0 & 0
\end{tabular} & 0 & 0 & 11 & 0 & 0 & 0 & 0 & 0 & 0 & 0 & 0 \\
\hline $2139 / 99$ & nicht aufgekl. & 0 & 0 & 2 & 1 & 23 & 30 & 0 & 0 & \begin{tabular}{l|l}
0 & 8
\end{tabular} & 0 & 0 & 11 & 0 & 0 & 0 & 0 & 0 & \begin{tabular}{|l|}
0 \\
\end{tabular} & 0 & 0 \\
\hline $2160 / 99$ & nicht aufgekl. & 0 & 0 & 4 & 0 & \begin{tabular}{l|l}
0 & 0 \\
\end{tabular} & 0 & 0 & 0 & \begin{tabular}{l|l}
0 & 8
\end{tabular} & 0 & 0 & 0 & 0 & 0 & \begin{tabular}{|l|}
0 \\
\end{tabular} & 0 & 0 & 0 & 0 & 0 \\
\hline $59 / 00$ & nicht aufgekl. & 0 & 0 & 2 & 0 & \begin{tabular}{l|l}
0 & 0 \\
\end{tabular} & 0 & 0 & 0 & \begin{tabular}{l|l}
7 & 8
\end{tabular} & \begin{tabular}{l|l}
3 & 0
\end{tabular} & 0 & 0 & 0 & 0 & 0 & 0 & 0 & 0 & 0 & 0 \\
\hline $133 / 00$ & nicht aufgekl. & 0 & 0 & 2 & 1 & \begin{tabular}{l|l}
0 & 3
\end{tabular} & \begin{tabular}{|l|l}
3 & 4
\end{tabular} & 5 & 6 & \begin{tabular}{l|l}
0 & 0
\end{tabular} & 0 & 0 & 0 & 0 & 0 & 0 & 0 & 0 & 0 & 0 & 0 \\
\hline $161 / 00$ & natürl. & 0 & 0 & 2 & 1 & \begin{tabular}{l|l}
2 & 3 \\
\end{tabular} & \begin{tabular}{|l|l}
3 & 4 \\
\end{tabular} & 0 & 0 & \begin{tabular}{l|l}
0 & 0
\end{tabular} & 0 & 0 & 0 & 0 & 0 & 0 & 0 & 0 & 0 & 0 & 0 \\
\hline $232 / 00$ & nicht aufgekl. & 0 & Infl & 4 & 1 & \begin{tabular}{l|l}
2 & 3
\end{tabular} & \begin{tabular}{|l|l}
3 & 4
\end{tabular} & 5 & 0 & \begin{tabular}{l|l}
0 & 0
\end{tabular} & 0 & 0 & 11 & 0 & 0 & 0 & 0 & 0 & 0 & 0 & 0 \\
\hline $275 / 00$ & nicht aufgekl. & 0 & 0 & 1 & 0 & \begin{tabular}{l|l}
0 & 0 \\
\end{tabular} & 0 & 0 & 0 & \begin{tabular}{l|l}
0 & 0 \\
\end{tabular} & 0 & 0 & 0 & 0 & 0 & 0 & 0 & 0 & 0 & 0 & 0 \\
\hline $381 / 00$ & nicht aufgekl. & 0 & 0 & 2 & 1 & \begin{tabular}{l|l}
2 & 3 \\
\end{tabular} & \begin{tabular}{|l|l}
3 & 4 \\
\end{tabular} & 0 & $\begin{array}{ll}0 & \\
\end{array}$ & \begin{tabular}{l|l}
77 & 0 \\
\end{tabular} & 0 & 0 & 0 & 0 & 0 & 0 & 0 & 0 & 0 & 0 & 0 \\
\hline $393 / 00$ & $\#$ & 0 & 0 & 2 & 1 & $0 \mid 3$ & \begin{tabular}{|l|l}
3 & 4 \\
\end{tabular} & 0 & 6 & \begin{tabular}{l|l}
0 & 0
\end{tabular} & 9 & 0 & 11 & 0 & 0 & 0 & 0 & 0 & 0 & 0 & 0 \\
\hline $413 / 00$ & nicht aufgekl. & Strept & 0 & 4 & 0 & \begin{tabular}{l|l}
0 & 0 \\
\end{tabular} & 0 & 0 & 0 & $\begin{array}{ll}0 & 0\end{array}$ & 10 & 0 & 0 & 0 & 0 & 0 & 0 & \begin{tabular}{|l|}
0 \\
\end{tabular} & 0 & 0 & 0 \\
\hline $442 / 00$ & nicht nat. & 0 & 0 & 4 & 0 & \begin{tabular}{l|l}
0 & 0 \\
\end{tabular} & 0 & 0 & 0 & 0 & 0 & 0 & \begin{tabular}{|l|}
0 \\
\end{tabular} & 12 & 0 & \begin{tabular}{|l|}
0 \\
\end{tabular} & 0 & 16 & \begin{tabular}{|l|}
17 \\
\end{tabular} & 0 & 0 \\
\hline $500 / 00$ & nicht aufgekl. & 0 & 0 & 2 & 0 & & 0 & 5 & 0 & \begin{tabular}{l|l}
0 & 0 \\
\end{tabular} & 0 & 0 & 11 & 12 & 0 & 0 & 0 & 0 & 0 & 0 & 0 \\
\hline $759 / 00$ & nicht aufgekl. & 0 & 0 & 3 & 1 & & & 5 & 0 & & 9 & 0 & 11 & 0 & 0 & \begin{tabular}{|l|}
0 \\
\end{tabular} & 0 & \begin{tabular}{|l|}
0 \\
\end{tabular} & \begin{tabular}{|l|}
0 \\
\end{tabular} & 0 & \\
\hline
\end{tabular}




\begin{tabular}{|c|c|c|c|c|c|c|c|c|c|c|c|c|c|c|c|c|c|c|c|c|c|}
\hline GS-Nr. & $\begin{array}{l}\text { Todesart It. } \\
\text { Todesbe- } \\
\text { scheinigung }\end{array}$ & $\begin{array}{c}\text { Bakterio- } \\
\text { logie }\end{array}$ & $\begin{array}{l}\text { Viro- } \\
\text { logie }\end{array}$ & $\begin{array}{l}\text { Kate- } \\
\text { gorie }\end{array}$ & \multicolumn{17}{|c|}{ Histologische Befunde } \\
\hline $824 / 00$ & nicht aufgekl. & 0 & 0 & 3 & & 2 & & & & 0 & \begin{tabular}{l|l}
0 & 0
\end{tabular} & 0 & 0 & 0 & 0 & 0 & 0 & 16 & 0 & 0 & 0 \\
\hline $896 / 00$ & nicht aufgekl. & 0 & 0 & 1 & 0 & 0 & 0 & 0 & 0 & 0 & \begin{tabular}{l|l}
0 & 0
\end{tabular} & 0 & 0 & 0 & 0 & 0 & 0 & 0 & 0 & 0 & 0 \\
\hline $986 / 00$ & nicht aufgekl. & 0 & 0 & 4 & 1 & 2 & 36 & 5 & 0 & 0 & \begin{tabular}{l|l}
0 & 0
\end{tabular} & 0 & 0 & 0 & 0 & 0 & 0 & 16 & 0 & 0 & 0 \\
\hline $1015 / 00$ & nicht aufgekl. & 0 & 0 & 2 & 0 & 0 & $3 \mid 2$ & 5 & 0 & 0 & \begin{tabular}{l|l}
0 & 0
\end{tabular} & 0 & 0 & 0 & 0 & 0 & 0 & 0 & 0 & 0 & 0 \\
\hline $1069 / 00$ & nicht aufgekl. & 0 & 0 & 4 & 1 & 0 & 36 & to & 0 & 0 & \begin{tabular}{l|l}
0 & 0
\end{tabular} & 0 & 0 & 12 & 0 & 0 & 0 & 16 & 0 & 0 & 0 \\
\hline $1134 / 00$ & nicht aufgekl. & Mening & 0 & 4 & 1 & 0 & $0 \mathrm{c}$ & To & 6 & 0 & \begin{tabular}{l|l}
0 & 0
\end{tabular} & 0 & 0 & 12 & 0 & 0 & 0 & 16 & 0 & 0 & 0 \\
\hline $1331 / 00$ & nicht aufgekl. & 0 & 0 & 2 & 0 & 0 & 0 & 5 & 0 & 7 & \begin{tabular}{l|l}
0 & 0
\end{tabular} & 0 & 11 & 0 & 0 & 0 & 0 & 16 & 0 & 0 & 0 \\
\hline $1333 / 00$ & nicht aufgekl. & 0 & 0 & 2 & 0 & 0 & $0 \mathrm{c}$ & 5 & 0 & 7 & \begin{tabular}{l|l}
0 & 0
\end{tabular} & 0 & 0 & 12 & 0 & 0 & 0 & 0 & 0 & 0 & 0 \\
\hline $1371 / 00$ & nicht aufgekl. & Staph & 0 & $\#$ & \# & $\#$ & $\# \neq$ & \# & $\#$ & $\#$ & $\#$ \# & $\#$ & $\#$ & $\#$ & $\#$ & $\#$ & $\#$ & $\#$ & $\#$ & $\#$ & $\#$ \\
\hline $1687 / 00$ & nicht aufgekl. & 0 & 0 & 2 & 1 & 0 & $3 \mathrm{C}$ & 0 & 0 & 0 & $\begin{array}{lll}0 & 0 \\
\end{array}$ & 0 & 11 & 0 & 0 & 0 & 0 & 0 & 0 & 0 & 0 \\
\hline $1768 / 00$ & nicht aufgekl. & \begin{tabular}{|c|} 
Strept,Menin \\
$\mathrm{g}$
\end{tabular} & 0 & 3 & 1 & 0 & 0 & 5 & 6 & 0 & \begin{tabular}{l|l}
0 & 9
\end{tabular} & 0 & 11 & 0 & 0 & 0 & 0 & 16 & 0 & 0 & 0 \\
\hline $1789 / 00$ & nicht aufgekl. & Strept & 0 & 4 & 0 & 0 & 0 c & 5 & 0 & 0 & \begin{tabular}{l|l}
0 & 0 \\
\end{tabular} & 0 & \begin{tabular}{|l|} 
\\
\end{tabular} & 0 & 0 & 14 & 0 & 16 & 0 & 18 & 19 \\
\hline $1814 / 00$ & nicht aufgekl. & Strept & 0 & 4 & 0 & 0 & $0 \mathrm{C}$ & 5 & 6 & 0 & \begin{tabular}{l|l}
0 & 0
\end{tabular} & \begin{tabular}{|l}
0 \\
\end{tabular} & 11 & 0 & 0 & 0 & 0 & 0 & 0 & 0 & 0 \\
\hline $1857 / 00$ & nicht aufgekl. & 0 & 0 & 3 & 1 & 0 & \begin{tabular}{l|l}
3 & 2 \\
&
\end{tabular} & To & 6 & 0 & \begin{tabular}{l|l}
0 & 0
\end{tabular} & 0 & 0 & 0 & 0 & 0 & 0 & 16 & 0 & 0 & 0 \\
\hline $2116 / 00$ & nicht aufgekl. & 0 & 0 & 2 & 0 & 0 & $0] \mathrm{c}$ & 0 & 0 & 7 & \begin{tabular}{l|l}
8 & 0 \\
\end{tabular} & 0 & 0 & 0 & 0 & 0 & 0 & 0 & 0 & 0 & 0 \\
\hline $2180 / 00$ & nicht aufgekl. & 0 & 0 & 3 & 7 & 2 & 3 & 10 & 0 & 0 & \begin{tabular}{l|l}
0 & 0
\end{tabular} & 0 & 11 & 0 & 0 & 0 & 0 & \begin{tabular}{|l|}
0 \\
\end{tabular} & 0 & 0 & 0 \\
\hline $2201 / 00$ & nicht aufgekl. & Strept & 0 & 2 & 1 & 0 & 0 & 0 & 0 & 0 & \begin{tabular}{l|l}
0 & 0 \\
\end{tabular} & 0 & 0 & 0 & 0 & 0 & 0 & 16 & 0 & 0 & 0 \\
\hline $138 / 01$ & nicht aufgekl. & Staph & 0 & 2 & 1 & 0 & $3 \mid 2$ & To & 6 & 7 & \begin{tabular}{l|l}
0 & 0
\end{tabular} & 0 & 0 & 0 & 0 & 0 & 0 & 0 & 0 & 0 & 0 \\
\hline $145 / 01$ & nicht aufgekl. & 0 & CMV & 4 & 1 & 0 & 30 & 0 & 0 & 7 & \begin{tabular}{l|l}
0 & 0 \\
\end{tabular} & 10 & 0 & 0 & 0 & 0 & 0 & 0 & 17 & 0 & 0 \\
\hline $309 / 01$ & $\#$ & 0 & CMV,RS & 3 & 0 & 0 & 32 & 0 & 0 & 0 & \begin{tabular}{l|l}
0 & 0 \\
\end{tabular} & 0 & 11 & 0 & 0 & 0 & 0 & 0 & 0 & 0 & 0 \\
\hline $381 / 01$ & nicht aufgekl. & \# & CMV & 4 & 0 & 0 & 0 & 10 & 0 & 0 & \begin{tabular}{l|l}
0 & 0
\end{tabular} & 0 & 11 & 0 & 0 & 0 & 0 & \begin{tabular}{|l|}
0 \\
\end{tabular} & 0 & 0 & 0 \\
\hline $493 / 01$ & nicht aufgekl. & Staph & & 2 & 0 & 0 & $0]$ & 0 & 0 & 7 & \begin{tabular}{l|l}
0 & 0 \\
\end{tabular} & 0 & 0 & 0 & 0 & 0 & 0 & 16 & 0 & 0 & 0 \\
\hline $565 / 01$ & nicht aufgekl. & Staph,Strept & & 2 & 1 & 0 & 0 c & To & 0 & 0 & \begin{tabular}{l|l}
0 & 0 \\
\end{tabular} & 0 & 0 & 0 & 0 & 0 & 0 & 0 & 0 & 0 & 0 \\
\hline $576 / 01$ & nicht aufgekl. & 0 & & 2 & 1 & 0 & 36 & 5 & 6 & 0 & \begin{tabular}{l|l}
0 & 0
\end{tabular} & 0 & 0 & 0 & 0 & 0 & 0 & 0 & 0 & 0 & 0 \\
\hline $801 / 01$ & nicht aufgekl. & 0 & & 2 & 1 & 2 & 36 & To & 0 & 0 & \begin{tabular}{l|l}
0 & 0
\end{tabular} & 0 & 0 & 0 & 0 & 0 & 0 & 0 & 0 & 0 & 0 \\
\hline $889 / 01$ & natürl. & 0 & Herpes & 4 & 1 & 0 & 36 & 10 & 6 & 7 & \begin{tabular}{l|l}
0 & 0
\end{tabular} & 0 & 11 & 12 & 0 & 0 & 0 & 0 & 0 & 0 & 0 \\
\hline $961 / 01$ & nicht aufgekl. & 0 & & 2 & 0 & 0 & 0 & 0 & 6 & 0 & \begin{tabular}{l|l}
0 & 0 \\
\end{tabular} & 0 & 0 & 0 & 0 & 0 & 0 & 0 & 0 & 0 & 0 \\
\hline $980 / 01$ & nicht aufgekl. & Kleb & & 2 & 0 & 2 & 30 & 0 & 0 & 0 & \begin{tabular}{l|l}
0 & 0 \\
\end{tabular} & 0 & 0 & 0 & 0 & 0 & 0 & 0 & 0 & 0 & 0 \\
\hline $1096 / 01$ & nicht aufgekl. & 0 & & 2 & 0 & 2 & 0 & 0 & 0 & 0 & \begin{tabular}{l|l}
0 & 0 \\
\end{tabular} & 0 & 0 & 12 & 0 & 0 & 0 & 0 & 0 & 0 & 0 \\
\hline $1154 / 01$ & nicht aufgekl. & Staph,Strept & & 3 & 1 & 2 & 30 & 0 & 0 & 7 & \begin{tabular}{l|l}
0 & 0 \\
\end{tabular} & 0 & 11 & 12 & 0 & 0 & 0 & 0 & 0 & 0 & 19 \\
\hline $1171 / 01$ & nicht aufgekl. & 0 & & 2 & 0 & 2 & 0 & 10 & 0 & 0 & \begin{tabular}{l|l}
0 & 0
\end{tabular} & 0 & 0 & 12 & 0 & 0 & 0 & \begin{tabular}{|l|}
0 \\
\end{tabular} & 0 & 0 & 0 \\
\hline $1355 / 01$ & nicht aufgekl. & 0 & & 2 & 1 & 0 & 0 & 5 & 6 & 0 & \begin{tabular}{l|l}
8 & 0 \\
\end{tabular} & 0 & 0 & 0 & 0 & 0 & 0 & 0 & 0 & 0 & 0 \\
\hline $1456 / 01$ & nicht aufgekl. & Strept & & 2 & 1 & 0 & $0 \mathrm{c}$ & 10 & 0 & 0 & \begin{tabular}{l|l}
0 & 0
\end{tabular} & 0 & 11 & 0 & 0 & 0 & 0 & 0 & 0 & 0 & 0 \\
\hline $1554 / 01$ & nicht aufgekl. & 0 & & 3 & 1 & 0 & 0 (c & 5 & 0 & 0 & \begin{tabular}{l|l}
0 & 0
\end{tabular} & 0 & 0 & 0 & 0 & 0 & 0 & 0 & 0 & 0 & 0 \\
\hline $1746 / 01$ & nicht aufgekl. & Staph & & 2 & 1 & & 36 & To & 6 & 0 & \begin{tabular}{l|l}
0 & 0
\end{tabular} & 0 & 0 & 0 & 0 & 0 & 0 & \begin{tabular}{|l|}
0 \\
\end{tabular} & 0 & 0 & 0 \\
\hline $1923 / 01$ & nicht aufgekl. & Staph & & 4 & 1 & & & & 6 & 0 & \begin{tabular}{l|l}
0 & 0
\end{tabular} & 0 & 0 & 12 & 0 & 0 & 0 & \begin{tabular}{|l|}
0 \\
\end{tabular} & 0 & 0 & 19 \\
\hline
\end{tabular}

Legende zu den histologischen Befunden:

$\begin{array}{llllll}1 & \text { Laryngitits } & 2 & \text { Rhinitis/ Pharyngitis } & 3 & \text { Tracheitis } \\ 4 & \text { Bronchi(oli)tis } & 5 & \text { Pneumonie } & 6 & \text { Tonsillitis } \\ 7 & \text { Sialadenitis } & 8 & \text { Aspiration } & 9 & \text { Gastritis } \\ 10 & \text { Hyaline Membranen } & 11 & \text { Enteritis } & 12 & \text { Kolitis } \\ 13 & \text { Appendizitis } & 14 & \text { Nephritis } & 15 & \text { Tubulusnekrose } \\ 16 & \text { Hepatitis } & 17 & \text { Pankreatitis } & 18 & \text { Organverfettung } \\ 19 & \text { Endo-/Myokarditis } & & & & \end{array}$


Tabelle 79:

\begin{tabular}{|c|c|c|c|c|c|c|}
\hline GS-Nr. & $\begin{array}{l}\text { Postmortales } \\
\text { Intervall bis } \\
\text { Sektion }\end{array}$ & $\begin{array}{l}\text { Rea- } \\
\text { nima- } \\
\text { tion }\end{array}$ & \begin{tabular}{|c|} 
Intervall \\
"lebend \\
gesehen" / \\
"tot aufge- \\
funden" \\
\end{tabular} & $\begin{array}{l}\text { Geschwis- } \\
\text { ter Anzahl }\end{array}$ & $\begin{array}{l}\text { Beruf Mutter } \\
\text { (Kategorie) }\end{array}$ & $\begin{array}{l}\text { Beruf Vater } \\
\text { (Kategorie) }\end{array}$ \\
\hline $171 / 99$ & 26 & ja & 1 & 0 & 1 & 1 \\
\hline $175 / 99$ & 38 & ja & 7 & 2 & 1 & 3 \\
\hline $181 / 99$ & 17 & ja & 11 & \# & 1 & 3 \\
\hline $262 / 99$ & 44 & ja & 12 & 1 & 1 & 3 \\
\hline $284 / 99$ & & nein & $\#$ & & \# & \\
\hline $511 / 99$ & \# & \# & $15 \mathrm{~min}$ & 1 & 2 & \# \\
\hline $602 / 99$ & $\#$ & $\#$ & 8 & 1 & 2 & 3 \\
\hline $760 / 99$ & $\#$ & $\#$ & $\#$ & 1 & 4 & 3 \\
\hline $781 / 99$ & $\#$ & $\#$ & 2 & $\#$ & \# & \# \\
\hline $852 / 99$ & $\#$ & $\#$ & 6 & $\#$ & $\#$ & $\#$ \\
\hline $914 / 99$ & $\#$ & $\#$ & 1 & 1 & 3 & 3 \\
\hline $950 / 99$ & $\#$ & $\#$ & 8 & 0 & 1 & 1 \\
\hline $981 / 99$ & $\#$ & $\#$ & 4 & 2 & 3 & $\#$ \\
\hline $1018 / 99$ & $\#$ & $\#$ & 8 & Zwilling, 2 & 1 & $\#$ \\
\hline $1079 / 99$ & \# & $\#$ & 1 & 1 & \# & $\#$ \\
\hline $1080 / 99$ & $\#$ & $\#$ & $\#$ & $\#$ & $\#$ & $\#$ \\
\hline $1155 / 99$ & $\#$ & $\#$ & 1 & 0 & 1 & 2 \\
\hline $1184 / 99$ & $\#$ & $\#$ & 10 & 7 & 1 & 1 \\
\hline $1195 / 99$ & & \# & 10 & 0 & 3 & 3 \\
\hline $1258 / 99$ & 37 & ja & $\#$ & $\#$ & $\#$ & \# \\
\hline $1459 / 99$ & 66 & nein & 2 & 0 & 1 & $\#$ \\
\hline $1461 / 99$ & $\#$ & $\#$ & 2 & 0 & $\#$ & $\#$ \\
\hline $1537 / 99$ & 8 & ja & 3 & 1 & 1 & 3 \\
\hline $1624 / 99$ & 59 & ja & 4 & Zwilling & 1 & 3 \\
\hline $1625 / 99$ & 62 & $\mathrm{ja}$ & 14 & 1 & 1 & 2 \\
\hline $1645 / 99$ & 28 & nein & 3 & 2 & 1 & $\#$ \\
\hline $1685 / 99$ & $\#$ & $\#$ & 3 & 1 & 4 & \# \\
\hline $1821 / 99$ & \# & $\#$ & 2 & 0 & 1 & 2 \\
\hline $1862 / 99$ & $\#$ & $\#$ & 5 & 0 & 1 & 3 \\
\hline $1891 / 99$ & 36 & nein & 9 & 1 & 3 & $\#$ \\
\hline $1961 / 99$ & $\#$ & $\#$ & 9 & 1 & 2 & 3 \\
\hline $2080 / 99$ & 85 & nein & 10 & 3 & 1 & 2 \\
\hline $2133 / 99$ & 5 & ja & 1 & 0 & 3 & 3 \\
\hline $2139 / 99$ & 38 & nein & 5 & $\#$ & 3 & 3 \\
\hline $2160 / 99$ & $\#$ & $\#$ & 5 & 3 & 2 & \# \\
\hline $59 / 00$ & 29 & ja & 3 & 0 & 1 & 2 \\
\hline $133 / 00$ & 16 & nein & 4 & 1 & 1 & $\#$ \\
\hline $161 / 00$ & 60 & nein & 7 & 1 & 2 & 4 \\
\hline $232 / 00$ & $\#$ & $\#$ & \# & $\#$ & $\#$ & \# \\
\hline $275 / 00$ & 13 & nein & 6 & 1 & 2 & 1 \\
\hline $381 / 00$ & 26 & nein & 2 & 3 & 1 & 3 \\
\hline $393 / 00$ & 18 & nein & 1 & 1 & 3 & 4 \\
\hline $413 / 00$ & $\#$ & $\#$ & $\#$ & 2 & $\#$ & $\#$ \\
\hline $442 / 00$ & 42 & nein & $10 \mathrm{~min}$ & 1 & 1 & 2 \\
\hline $500 / 00$ & 31 & ja & 1 & 0 & 1 & 1 \\
\hline
\end{tabular}




\begin{tabular}{|c|c|c|c|c|c|c|}
\hline GS-Nr. & $\begin{array}{l}\text { Postmortales } \\
\text { Intervall bis } \\
\text { Sektion }\end{array}$ & $\begin{array}{c}\text { Rea- } \\
\text { nima- } \\
\text { tion }\end{array}$ & $\begin{array}{c}\text { Intervall } \\
\text { "lebend } \\
\text { gesehen" / } \\
\text { "tot aufge- } \\
\text { funden" } \\
\end{array}$ & $\begin{array}{l}\text { Geschwis- } \\
\text { ter Anzahl }\end{array}$ & $\begin{array}{l}\text { Beruf Mutter } \\
\text { (Kategorie) }\end{array}$ & $\begin{array}{l}\text { Beruf Vater } \\
\text { (Kategorie) }\end{array}$ \\
\hline $759 / 00$ & 14 & nein & 9 & 1 & 1 & 3 \\
\hline $824 / 00$ & 100 & nein & 3 & 1 & 1 & 2 \\
\hline $896 / 00$ & 30 & nein & 3 & 1 & 1 & 3 \\
\hline $986 / 00$ & 57 & ja & $\#$ & Zwilling, 2 & 1 & 4 \\
\hline $1015 / 00$ & 42 & ja & 10 & 1 & 1 & 3 \\
\hline $1069 / 00$ & 37 & ja & $\#$ & $\#$ & $\#$ & $\#$ \\
\hline $1134 / 00$ & $\#$ & $\#$ & $\#$ & 0 & 1 & 2 \\
\hline $1331 / 00$ & 57 & ja & 6 & 3 & $\#$ & $\#$ \\
\hline $1333 / 00$ & 56 & nein & 5 & 2 & 1 & 2 \\
\hline $1371 / 00$ & $\#$ & $\#$ & 8 & 0 & 1 & $\#$ \\
\hline $1687 / 00$ & 16 & nein & 10 & 1 & 3 & 3 \\
\hline $1768 / 00$ & 10 & ja & 5 & 0 & 2 & 2 \\
\hline $1789 / 00$ & 34 & nein & 5 & 2 & $\#$ & 3 \\
\hline $1814 / 00$ & 72 & ja & $\#$ & 0 & 1 & 4 \\
\hline $1857 / 00$ & 32 & ja & 5 & 0 & 3 & $\#$ \\
\hline $2116 / 00$ & 49 & nein & 5 & 2 & 1 & 2 \\
\hline $2180 / 00$ & 16 & nein & 10 & 1 & 1 & $\#$ \\
\hline $2201 / 00$ & 14 & ja & 8 & 0 & $\#$ & $\#$ \\
\hline $138 / 01$ & 46 & ja & 1 & 2 & $\#$ & $\#$ \\
\hline $145 / 01$ & 95 & ja & $\#$ & 0 & $\#$ & $\#$ \\
\hline $309 / 01$ & 32 & nein & 3 & 1 & $\#$ & $\#$ \\
\hline $381 / 01$ & 11 & ja & $15 \mathrm{~min}$ & 0 & 1 & 1 \\
\hline $493 / 01$ & 6 & ja & 2 & 1 & 1 & 2 \\
\hline $565 / 01$ & 16 & nein & 3 & 1 & 1 & 2 \\
\hline $576 / 01$ & 8 & nein & 2 & 3 & 1 & $\#$ \\
\hline $801 / 01$ & 76 & nein & 1 & 1 & 1 & 3 \\
\hline $889 / 01$ & 29 & ja & $\#$ & $\#$ & $\#$ & $\#$ \\
\hline $961 / 01$ & 95 & ja & $\#$ & 1 & 1 & $\#$ \\
\hline $980 / 01$ & 29 & ja & 4 & 2 & 1 & 3 \\
\hline $1096 / 01$ & 68 & ja & 12 & 1 & $\#$ & $\#$ \\
\hline $1154 / 01$ & 34 & ja & $\#$ & $\#$ & $\#$ & $\#$ \\
\hline $1171 / 01$ & 37 & nein & 7 & 1 & 1 & 1 \\
\hline $1355 / 01$ & 63 & ja & $\#$ & $\#$ & $\#$ & $\#$ \\
\hline $1456 / 01$ & $\#$ & $\#$ & 1 & Zwilling, 2 & 1 & $\#$ \\
\hline $1554 / 01$ & $\#$ & $\#$ & 5 & $\#$ & $\#$ & $\#$ \\
\hline $1746 / 01$ & 29 & ja & 3 & 1 & 1 & 3 \\
\hline $1923 / 01$ & $\#$ & $\#$ & $\#$ & $\#$ & $\#$ & $\#$ \\
\hline
\end{tabular}




\section{Begleitstoffanalysen}

Tabelle 80:

\begin{tabular}{|c|c|c|c|c|c|c|c|c|c|c|c|}
\hline -Nr. & $\begin{array}{l}\text { Kate- } \\
\text { gorie }\end{array}$ & $\begin{array}{c}\text { Rea- } \\
\text { nima- } \\
\text { tion }\end{array}$ & $\begin{array}{c}\text { post- } \\
\text { morta- } \\
\text { les } \\
\text { Inter- } \\
\text { vall } \\
\end{array}$ & $\begin{array}{c}\text { Alter } \\
\text { in } \\
\text { Mona- } \\
\text { ten }\end{array}$ & $\begin{array}{c}\text { Serum- } \\
\text { Metha- } \\
\text { nol }\end{array}$ & $\begin{array}{c}\text { Serum- } \\
\text { Propa- } \\
\text { nol-1 }\end{array}$ & \begin{tabular}{|c} 
Serum- \\
Butanol \\
-1
\end{tabular} & $\begin{array}{l}\text { Serum- } \\
\text { Acetal- } \\
\text { dehyd }\end{array}$ & $\begin{array}{l}\text { Serum- } \\
\text { Aceton }\end{array}$ & $\begin{array}{c}\text { Serum- } \\
\text { Isopro- } \\
\text { panol }\end{array}$ & $\begin{array}{l}\text { Ser } \\
\text { Etha }\end{array}$ \\
\hline $39 / 99$ & 2 & ein & 38 & 7 & 46 & 2,18 & \# & 5,28 & 1,58 & 0,92 & 0,354 \\
\hline $133 / 99$ & - & ja & 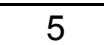 & 4 & 80 & \# & \# & 46 &, 53 & 11 & 0,00980 \\
\hline $2080 / 99$ & 4 & nein & 85 & 11 & 50 & 0,00 & \# & 1,21 & 35 & 11 & 0,00247 \\
\hline $1891 / 99$ & 2 & in & 36 & 10 & 20 & 0,12 & \# & 2,21 & 28 & 52 & 0,00990 \\
\hline \begin{tabular}{|c|}
$1645 / 99$ \\
\end{tabular} & 2 & ein & 28 & 7 & 64 & \# & \# & 1,36 & 61 & 72 & 0,00279 \\
\hline \begin{tabular}{|c|}
$1625 / 99$ \\
\end{tabular} & 2 & ja & 62 & 9 & 64 & 0,21 & 0,00 & 64 & 87 & 87 & 0,11243 \\
\hline \begin{tabular}{|l|}
$1624 / 99$ \\
\end{tabular} & 2 & ja & 59 & 4 & 1,02 & 0,07 & \# & 1,15 & 23 & 45 & 3793 \\
\hline $1537 / 99$ & 2 & ja & 8 & 5 & 1,64 & 13,00 & \# & 4,14 &, 28 & 41 & 0,06643 \\
\hline $1258 / 99$ & 4 & ja & 37 & 11 & 1,21 & 0,07 & \# & 1,43 & 14 & 36 & 0,00500 \\
\hline \begin{tabular}{|l|}
$1459 / 99$ \\
\end{tabular} & 2 & nein & 66 & 10 & 2,14 & \# & \# & 2,21 & 93 & 78 & 0,00393 \\
\hline $2201 / 00$ & 2 & nein & 14 & 11 & 2,60 & 0,48 & 0,00 & 1,10 & 2,89 & 33 & 0,00810 \\
\hline $2180 / 00$ & 3 & ein & 16 & 3 & 1,45 & 0,00 &, 05 & 0,72 & 25 & 24 & 550 \\
\hline 211 & 2 & nein & 49 & 5 & 3,59 & 0,08 &, 00 & & 52 & 25 & \\
\hline $1857 / 00$ & 8 & & 32 & 11 & 70 & 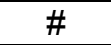 & 0,07 & & 57 & 96 & \\
\hline \begin{tabular}{|l|}
$1814 / 00$ \\
\end{tabular} & 4 & Jc & 72 & 8 & 8 & \# & \# & 30 & 91 & 13 & 760 \\
\hline \begin{tabular}{|l|}
$1768 / 00$ \\
\end{tabular} & 3 & ja & 10 & 4 & 1,14 & 0,06 & $\#$ & 1,73 & 86 & 44 & 0710 \\
\hline $1687 / 00$ & 2 & nein & 1 & 8 & 1,18 & 0,42 & \# & 2,03 & 0,99 & 23 & 0,09215 \\
\hline $1333 / 00$ & 2 & nein & 56 & 3 & 0,96 & \# & \# & 3,64 & 00 & 34 & 0,00464 \\
\hline $1331 / 00$ & 2 & ja & 57 & 3 & 21 & 0,04 & $\#$ & 5,94 & 64 & 41 & 0,00171 \\
\hline \begin{tabular}{|l|}
$1069 / 00$ \\
\end{tabular} & 4 & ja & 3 & - & 5 & 4 & 4 & 0,85 & 26 & 86 & 0,00221 \\
\hline 101 & 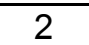 & ja & 4 & 1 & 1,85 & 09 & 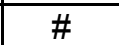 & 37 & 69 & 82 & 0,00980 \\
\hline \begin{tabular}{|l|}
$986 / 00$ \\
\end{tabular} & 4 & & 5 & 12 & 1,45 & 0,11 & 0,00 & 0,94 & 15 & 03 & \\
\hline \begin{tabular}{|l|}
$896 / 00$ \\
\end{tabular} & 1 & in & 3 & 6 & 1,37 & 0 & $\#$ & 9 & 24 & 52 & \\
\hline \begin{tabular}{|l|}
$824 / 00$ \\
\end{tabular} & 3 & 1 & 100 & 3 & 1,14 & 0,00 & \# & 2,26 & 54 & 61 & \\
\hline \begin{tabular}{|l|}
$759 / 00$ \\
\end{tabular} & 3 & nein & 14 & 11 & 11 & \# & 0,04 & 1,21 & 6,84 & 78 & 532 \\
\hline \begin{tabular}{|l|}
$500 / 00$ \\
\end{tabular} & 2 & ja & 31 & 4 & 8 & $\#$ & $\#$ & 1,36 & 0,39 & 22 & 190 \\
\hline $442 / 00$ & 4 & nein & 42 & 1 & 0,34 & 0,24 & \# & 0,74 & 0,31 & 98 & 0,01057 \\
\hline \begin{tabular}{|l|}
$393 / 00$ \\
\end{tabular} & 2 & & 1 & 4 & 0,76 & $\#$ & 0,57 & 0,79 & 1,23 & 0,28 & 0,00364 \\
\hline \begin{tabular}{|l|}
$381 / 00$ \\
\end{tabular} & 2 & & 2 & 2 & 0,89 & 0,64 & 0,57 & 1,80 & 0,47 & 1,12 & 0,26040 \\
\hline \begin{tabular}{|l|}
$275 / 00$ \\
\end{tabular} & 1 & $\mathrm{n}$ & 1 & 3 & 0,56 & 0,15 & 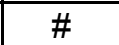 & 1,79 & 0,98 & 14 & 0,13107 \\
\hline \begin{tabular}{|l|}
$232 / 00$ \\
\end{tabular} & 4 & 1 & 60 & 10 & 0,94 & 0,77 & $\#$ & 75 & 10,40 & 71 & 0,22500 \\
\hline \begin{tabular}{|l|}
$161 / 00$ \\
\end{tabular} & 2 & & 6 & 9 & 1 & 0,07 & \# & 1,30 & 0,32 & 12 & 50 \\
\hline \begin{tabular}{|l|}
$133 / 00$ \\
\end{tabular} & 2 & nein & 1 & 10 & 1 & 1 & 0,07 & 5,91 & 3,79 & 97 & 307 \\
\hline $59 / 00$ & $\pi$ & 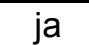 & 29 & 2 & 4 & 9 & $\pi$ & 2,80 & 1,70 & 71 & \\
\hline \begin{tabular}{|l|}
$1746 / 01$ \\
\end{tabular} & 2 & 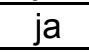 & 29 & 8 & 2,22 & 4,28 & 0,76 & 1,22 & 0,83 & 1,94 & 0,00290 \\
\hline \begin{tabular}{|l|}
$1355 / 01$ \\
\end{tabular} & 2 & ja & 63 & 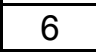 & 3,69 & 0,78 & 0,00 & 1,98 & 1,20 & 0,39 & 0,01614 \\
\hline \begin{tabular}{|l|}
$1171 / 01$ \\
\end{tabular} & 2 & nein & 37 & 5 & 0,79 & 0,00 & \# & 7,10 & 0,77 & 0,38 & 0,00600 \\
\hline $\mid 1154 / 01$ & 3 & ja & 34 & 0 & 2,85 & 0,00 & $\#$ & 2,30 & 0,70 & 0,28 & 0,00500 \\
\hline \begin{tabular}{|l|}
$1096 / 01$ \\
\end{tabular} & 2 & ja & 68 & 7 & 2,36 & 0,22 & H & 1,45 & 2,05 & 1,17 & 0,00593 \\
\hline & 2 & ja & 29 & 4 & 2,04 & 0,28 & \# & 1,30 & 1,07 & 0,28 & 0,10500 \\
\hline & 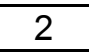 & Jo & 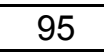 & 3 & 1,0 & 0,43 & 1,55 & 0,90 & 1,31 & 1,21 & 0,07607 \\
\hline $889 / 01$ & 4 & ja & 20 & 10 & 1,0 & \# & 0,00 & 1,53 & 3,18 & 0,97 & 0,00750 \\
\hline $801 / 01$ & 2 & nein & 76 & 7 & 2,14 & 0,14 & 0,07 & 7,57 & 1,33 & 0,86 & 0,018 \\
\hline
\end{tabular}




\begin{tabular}{|l|c|c|c|c|c|c|c|c|c|c|c|}
\hline GS-Nr. & $\begin{array}{c}\text { Kate- } \\
\text { gorie }\end{array}$ & $\begin{array}{c}\text { Rea- } \\
\text { nima- } \\
\text { tion }\end{array}$ & $\begin{array}{c}\text { post- } \\
\text { morta- } \\
\text { les } \\
\text { Inter- } \\
\text { vall }\end{array}$ & $\begin{array}{c}\text { Alter } \\
\text { in } \\
\text { Mona- } \\
\text { ten }\end{array}$ & $\begin{array}{c}\text { Serum- } \\
\text { Metha- } \\
\text { nol }\end{array}$ & $\begin{array}{c}\text { Serum- } \\
\text { Propa- } \\
\text { nol-1 }\end{array}$ & $\begin{array}{c}\text { Serum- } \\
\text { Butanol } \\
-1\end{array}$ & $\begin{array}{c}\text { Serum- } \\
\text { Acetal- } \\
\text { dehyd }\end{array}$ & $\begin{array}{c}\text { Serum- } \\
\text { Aceton }\end{array}$ & $\begin{array}{c}\text { Serum- } \\
\text { Isopro- } \\
\text { panol }\end{array}$ & $\begin{array}{c}\text { Serum- } \\
\text { Ethanol }\end{array}$ \\
\hline $576 / 01$ & 2 & nein & 8 & 5 & 1,40 & 5,20 & $\#$ & 1,60 & 10,00 & 1,90 & 0,30160 \\
\hline $565 / 01$ & 2 & nein & 16 & 5 & 0,71 & 0,28 & $\#$ & 2,31 & 0,65 & 0,18 & 0,20350 \\
\hline $493 / 01$ & 2 & ja & 6 & 2 & 1,07 & $\#$ & $\#$ & 0,60 & 0,37 & 0,42 & 0,00320 \\
\hline $381 / 01$ & 4 & ja & 11 & 8 & 0,92 & 0,10 & $\#$ & 0,58 & 1,98 & 0,61 & 0,00289 \\
\hline $309 / 01$ & 3 & nein & 32 & 6 & 1,17 & 0,00 & $\#$ & 4,26 & 1,50 & 1,04 & 0,00900 \\
\hline $145 / 01$ & 4 & ja & 95 & 1 & 1,21 & 0,00 & $\#$ & 2,90 & 1,86 & 0,64 & 0,00522 \\
\hline $138 / 01$ & 2 & ja & 46 & 5 & 1,40 & 0,04 & $\#$ & 0,55 & 0,93 & 0,50 & 0,00340 \\
\hline $1502 / 01$ & $\#$ & ja & 29 & 3 & 1,00 & 5,00 & $\#$ & 1,34 & 0,93 & 0,43 & 0,00291 \\
\hline $1772 / 01$ & $\#$ & nein & 37 & 7 & 1,71 & 0,04 & $\#$ & 1,27 & 1,65 & 0,61 & 0,12000 \\
\hline
\end{tabular}




\section{DANKSAGUNG}

\section{Mein „Danke schön“ gilt all denjenigen, die zum Gelingen dieser Arbeit beigetragen haben!}

Ich danke Herrn Prof. Dr. med. Wolfgang Eisenmenger, der diese Arbeit durch Einbindung des Instituts in die BMBF-Studie und großzügige finanzielle Unterstützung überhaupt ermöglichte.

Herrn Prof. Dr. med. Randolph Penning

danke ich sehr für die Überlassung des spannenden Themas, die immer freundliche (und unterhaltsame) Unterstützung, sowie das mir entgegengebrachte Vertrauen bezüglich der verantwortungsvollen Tätigkeiten für diese Studie.

Mein ganz besonderer Dank gilt Frau Dr. med. Elisabeth Rauch, die mir während der gesamten Zeit engagiert zur Seite stand, mich auch bei anderen wissenschaftlichen Betätigungen sehr gefördert und gefordert hat, und dadurch entscheidend an meiner beruflichen Zukunft mitgewirkt hat.

Ich danke Herrn Dr. med. Andreas Büttner für die wertvollen histologischen Nachhilfestunden und Miriam Finelli und Susanne Ring für die Hilfe und Einarbeitung in die histologische Technik.

Herrn Prof. Dr. med. Thomas Gilg und Frau Braun gilt mein Dank für die Durchführung und Hilfe bei den Alkoholbestimmungen und Begleitstoffanalysen, den Mitarbeitern der toxikologischen Abteilung für die durchgeführten Untersuchungen und den Herren Präparatoren Klein, Wieczorek, Kriner und Königer für die Unterstützung im Sektionssaal.

Meinen Eltern danke ich sehr für ihre Unterstützung, und meinem Ehemann Marc für seine Geduld an den unzähligen Abenden, an denen ich mit dieser Arbeit beschäftigt war.

\section{Vielen Dank!}




\section{LEBENSLAUF}

Bettina Michaela Zinka, geb. Böhme

geboren

Familienstand

Eltern

$1979-1983$

$1983-1988$

$1988-1993$

1993

$1994-1995$

1995

1998

1999

2000

2001

2002

2003

2004 am 18. Juni 1973 in Aachen

verheiratet mit Marc Zinka, Rechtsanwalt

keine Kinder

Doris Böhme, geb. Tillack, Dipl. Ökonomin

Dieter Böhme, Prof. Dr. Ing. Prof. h.c. Dr. h.c., Direktor der

Schweißtechnischen Lehr- und Versuchsanstalt SLV München

Besuch der Grundschulen in Orsoy und Moers, NRW

Besuch des Gymnasiums Rheinkamp in Moers, NRW

Besuch des mathematisch-naturwissenschaftlichen Gymnasiums in Pullach, Bayern

Abitur

Besuch der Heilpraktikerschule Lotz, München, Abendschule

Aufnahme des Studiums der Humanmedizin an der LudwigMaximilians-Universität (LMU) in München

Physikum am 19.08.1998

Aufnahme dieser Doktorarbeit und Anstellung als studentische Hilfskraft am Institut für Rechtsmedizin in München

Famulaturen in der Rechtsmedizin und Psychiatrie

Erstes Staatsexamen am 30. und 31.08.1999

Famulaturen in der Gynäkologie

Zweites Staatsexamen am 20. bis 24.08.2001

Praktisches Jahr vom 15.10.2001 bis 15.09.2002 mit Wahlfach

Psychiatrie

Drittes Staatsexamen am 14.10.2002

Aufnahme einer ÄIP-Tätigkeit an der Klinik für Psychiatrie und Psychotherapie der LMU

Aufnahme einer ÄIP-Tätigkeit am Pathologischen Institut der

LMU, Einreichen der Promotion

Mündliche Promotionsprüfung 